\title{
Resonant Excitation Spectroscopy and Photon Statistics of Self- assembled Semiconductor Quantum Dots
}

Disheng Chen

Follow this and additional works at: https://researchrepository.wvu.edu/etd

Part of the Physics Commons

\section{Recommended Citation}

Chen, Disheng, "Resonant Excitation Spectroscopy and Photon Statistics of Self-assembled Semiconductor Quantum Dots" (2017). Graduate Theses, Dissertations, and Problem Reports. 7992. https://researchrepository.wvu.edu/etd/7992

This Dissertation is protected by copyright and/or related rights. It has been brought to you by the The Research Repository @ WVU with permission from the rights-holder(s). You are free to use this Dissertation in any way that is permitted by the copyright and related rights legislation that applies to your use. For other uses you must obtain permission from the rights-holder(s) directly, unless additional rights are indicated by a Creative Commons license in the record and/ or on the work itself. This Dissertation has been accepted for inclusion in WVU Graduate Theses, Dissertations, and Problem Reports collection by an authorized administrator of The Research Repository @ WVU.

For more information, please contact researchrepository@mail.wvu.edu. 


\title{
Resonant Excitation Spectroscopy and Photon Statistics of Self-assembled Semiconductor Quantum Dots
}

\author{
Disheng Chen \\ Dissertation submitted \\ to the Eberly College of Arts and Sciences \\ at West Virginia University \\ in partial fulfillment of the requirements for the degree of \\ Doctor of Philosophy in \\ Physics \\ Edward B. Flagg, Ph.D., Chair \\ Alan D. Bristow, Ph.D. \\ Tudor D. Stanescu, Ph.D. \\ Lian Li, Ph.D. \\ Jeremy M. Dawson, Ph.D. \\ Department of Physics and Astronomy \\ Morgantown, West Virginia, United States of America \\ 2017
}

Keywords: Self-assembled Quantum Dot, Resonant Photoluminescence Excitation Spectroscopy, Secondorder Correlation Function, Density-Matrix Coherence, DC Stark Effect, Coherent Scattering

Copyright 2017 Disheng Chen 


\section{Abstract}

\section{Resonant Excitation Spectroscopy and Photon Statistics of Self-assembled Semiconductor Quantum Dots \\ Disheng Chen}

Light-matter interactions in semiconductor nanostructures have attracted significant research interest because of both fundamental physics questions and practical concerns. Epitaxially grown quantum dots (QDs), with their narrow emission linewidths and atom-like density of states in a solid state system, are archetypical elements of study and are potentially useful for many applications, such as on-demand single photon emitters [1,2], efficient entangled photon-pair sources [3,4], and cavity quantum electrodynamics (QED) research [5-11]. Most experiments employ resonant or near-resonant excitation to directly interact with the bound states, which enables high excitation efficiency, precise control of quantum states, and minimal disturbance of the local environment. However, the inevitable doping of the material in growth introduces an intrinsic free charge carrier reservoir that enables charge fluctuations of the QD and defects in its surrounding local environment. The direct consequences are fluorescent intermittency (or blinking) in a QD's emission, and spectral diffusion of the QD's energy level. Both effects pose challenges for using these photons as flying qubits to realize a quantum network or linear optical quantum computing. Blinking compromises the properties of these QDs useful for generating on-demand single photons. Characterizing these charge dynamics and understanding the underlying physics is critical for hunting potential methods to suppress them. In this dissertation, by examining the excitation spectra of the QD and the photon statistics of its emission, we are able to determine the possible trap locations and the time scale of these charge dynamics. In fact, the temporal correlation measurement captures both the non-classical nature of these quantum emitters and the charge dynamics of both the QD and nearby defects. This information helps identify the nature of these charge traps, and provides the clues for suppressing these electric fluctuations; for example, by modifying the sample growth parameters or fabricating additional nano-structures on the sample to deplete the free charge carriers.

One solution to these problems is to use a better sample with less intrinsic doping. It has been demonstrated that the photons emitted from the same QD in rapid succession can have very high indistinguishability when the QD is in an optical cavity [12,13] or is excited resonantly [14]. However, photons spaced widely in time and those from separate QDs do not show the same degree of indistinguishability [15-17] due to the inhomogeneous distribution of photon energies emitted by one QD state at different times. Considering that the state-of-art growth technique cannot achieve zero-doping growth, nor realize zero defect production, other methods are preferred. One alternative is to use coherent scattering. In this dissertation, we propose a new single photon source, a 3-level V-system, which is potentially a better single photon source than a single 2-level system in terms of generating single photons with sub-natural line-width [18]. Our calculation implies that 3-level system can output coherent scattering with a purity as high as $90 \%$ while keeping the coherent scattering intensity at the maximum value that a single 2-level system can generate in practice. Our calculation predicts an unconventional excitation line shape from 3-level V-system, which is confirmed experimentally here. The analysis indicates that the interference between the coherent scatterings from two dipoles is the cause for this phenomenon. Any systems with a V-shaped energy structure and orthogonal dipole moments are expected to observe this phenomenon if two transitions are non-degenerate and with a splitting on the order of a single line width. 


\section{Acknowledgements}

I would like to express my sincere gratitude to my advisor, Prof. Dr. Edward B. Flagg, for his patient guidance, amiable encouragement, intellectual enlightenment and generous advice throughout my time as his student. As a professor, he showed me the path and prospects of being a scientific researcher with constant passion, curiosity, and dedication, which motivates me to master the physics and tackle the unknown. As a supervisor, he devoted considerate care on not only the academic job of his students, but also their future career as a person. These interactions alleviate the stress of being a Ph.D. candidate and make the pursuing journey more enjoyable and pleasant. I have been extremely lucky to have a supervisor who values the teaching and educating of new generation of physicists appreciably, and who engages in the commitments with substantial efforts. I deeply appreciate these practice since it not only conveys me immense physics knowledge, but most importantly sets a high standard for my future career.

I must express my special gratitude to Prof. Dr. Mikel B. Holcomb, who served as my advisor (former) for the first 4 years of my Ph.D. period at West Virginia University. She opened the door of research for me, and taught me the skills of exploration. She provided me the opportunities to work on various compelling projects in Lawrence Berkeley National Laboratory, where I recognized how complex a modern physical experiment can be, and appreciated the difficulty for human being to unfold a "mystery" of the nature.

I owe a great debt of gratitude to my family for their continued support and encouragement throughout my Ph.D. period. I was always amazed by the patience of my mother, father and brother who experienced all of the ups and downs of my research. I could not imagine the fulfillment of this dissertation without their generous love.

My sincere thanks also goes to the rest of my committee members: Dr. Alan Bristow, Dr. Tudor Stanescu, Dr. Lian Li, and Dr. Jeremy Dawson for their encouragement, perceptive comments, and insightful questions.

Last but not the least, I thank my fellow labmates in Semiconductor Quantum Optics Lab (SQuOL): Gary Lander, Samet Demircan, Cabot Zabriskie, and Raju KC for the stimulating discussions, for the sleepless nights we were working together before deadlines, and for all the fun we have had in the last three and half years. I thank my friends in Holcomb's Group: Dr. Srinivas Polisetty, Chih-Yeh Huang, Robbyn Trappen, Guerau Cabrera, and Dr. Jinling Zhou, for the memorable work on complex oxides. I am also grateful to Dr. Zhengjun Wang, Bingyi Cui and Peng Zheng for mutual support on academic practice and daily life.

Sincerely,

Disheng Chen

May 2017 


\section{Table of Contents}

TITLE

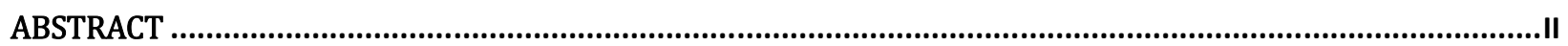

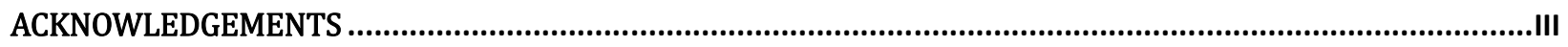

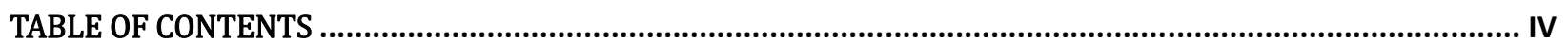

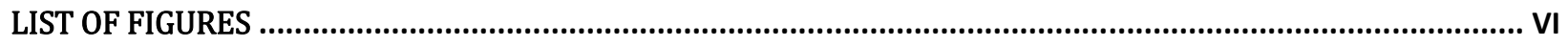

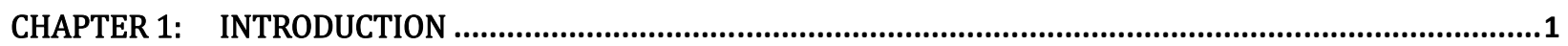

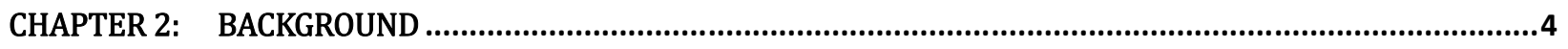

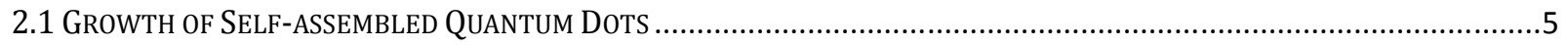

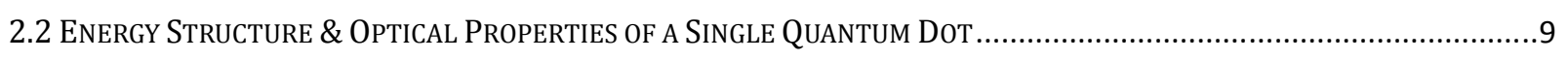

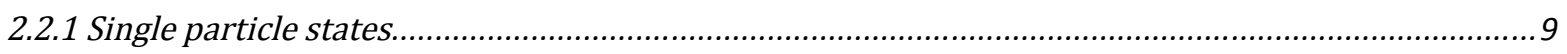

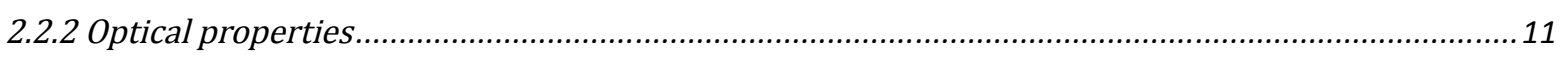

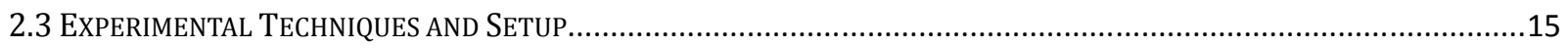

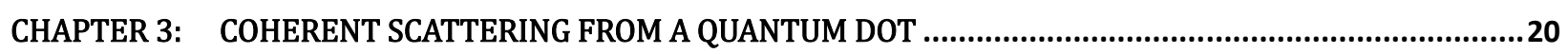

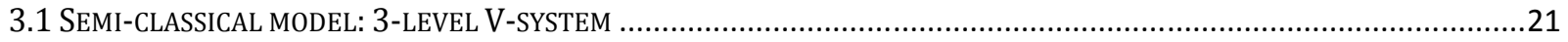

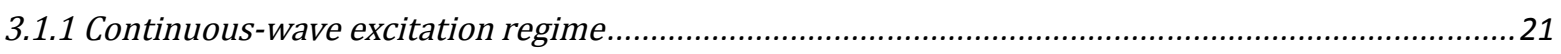

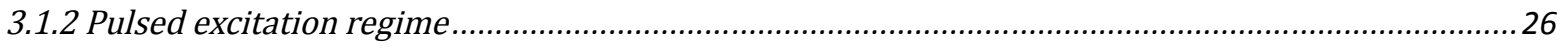

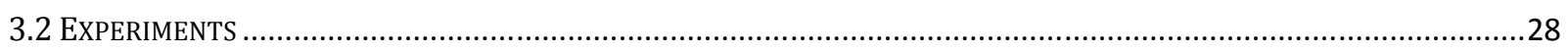

3.2.1 Carrier recombination in quantum dot: lifetime measurement .....................................................28

3.2.2 Resonant fluorescence excitation spectroscopy .............................................................................29

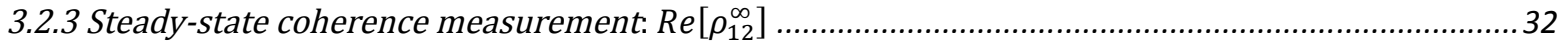

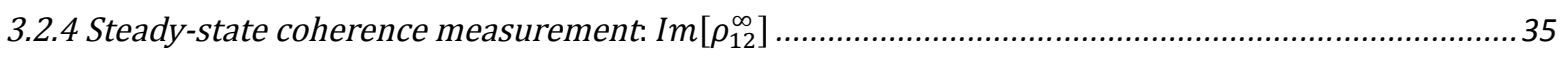

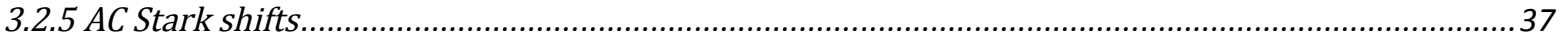

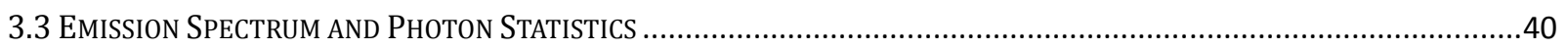

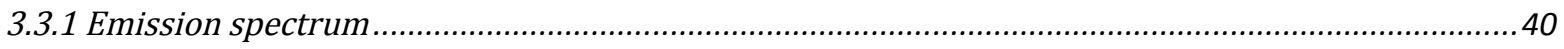

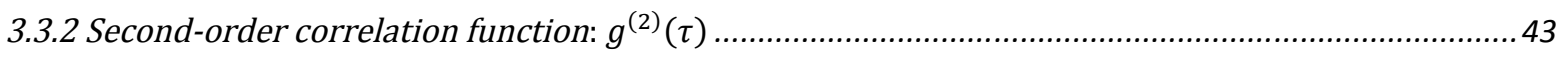

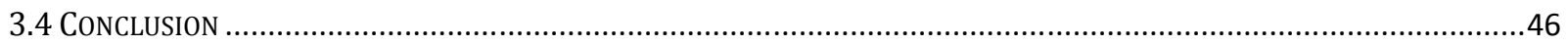

CHAPTER 4: ～LOCAL ELECTRIC ENVIRONMENT FLUCTUATION ....................................................................47

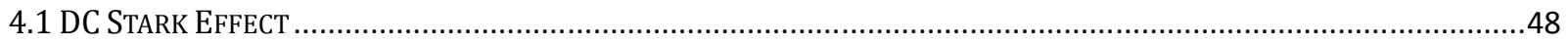

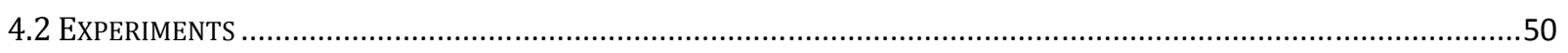

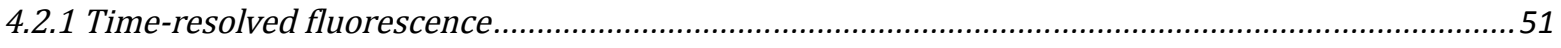

4.2.2 Resonant photoluminescence excitation spectroscopy ……...........................................................5

4.2.3 Correlation function of resonance fluorescence ............................................................................ 57

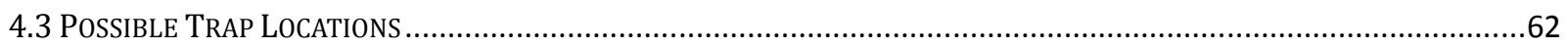

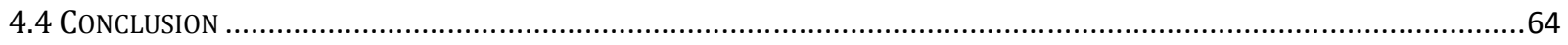




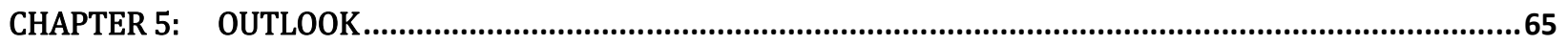

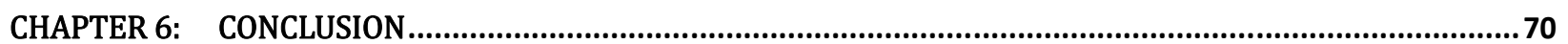

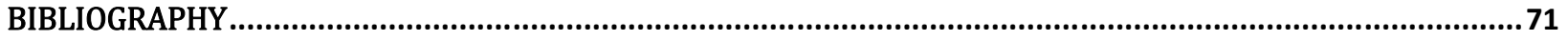

APPENDIX

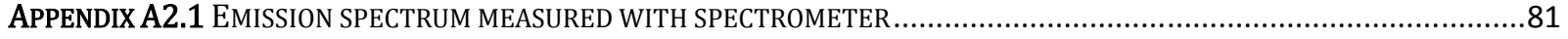

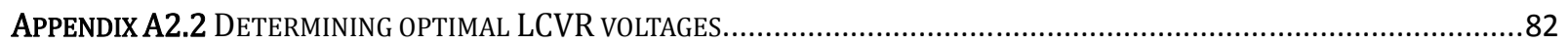

APPENDIX A2.3 MATLAB CODE FOR EXTRACTING SECOND-ORDER CORRELATION FUNCTION ..........................................85

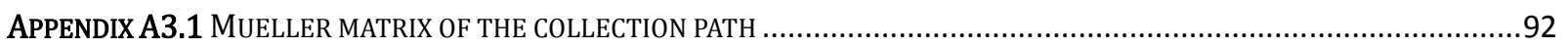

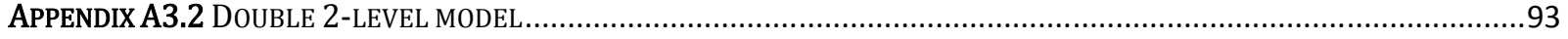

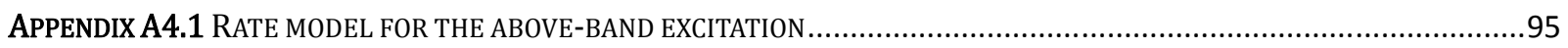

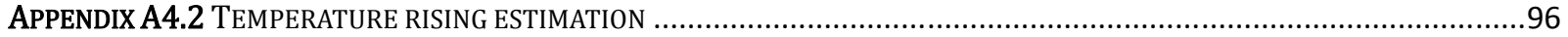




\section{List of Figures}

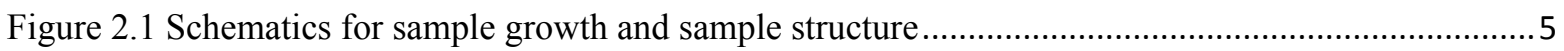

Figure 2.2 Transmittance of DBR mirrors and microcavity ............................................................ 6

Figure 2.3 Spatial image of PL from InGaAs quantum dots (QDs) ........................................................ 7

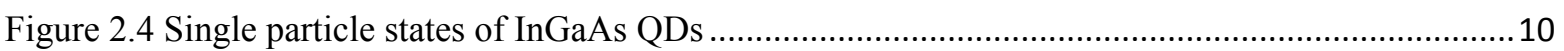

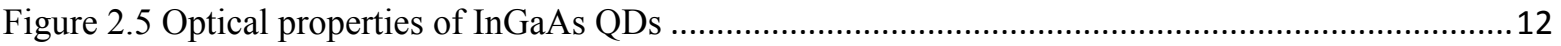

Figure 2.6 Optical setup for spectroscopy and correlation measurements..............................................16

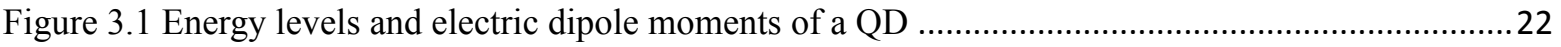

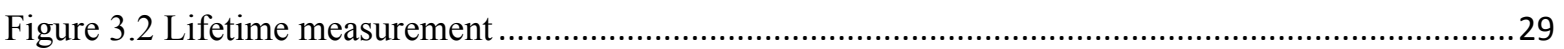

Figure 3.3 RPLE at different detection polarizations........................................................................ 30

Figure 3.4 Coherent scattering performance of a 3-level and a 2-level system ........................................31

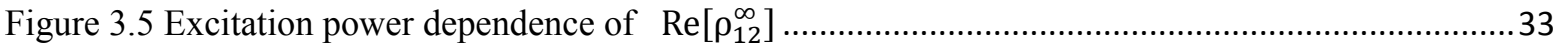

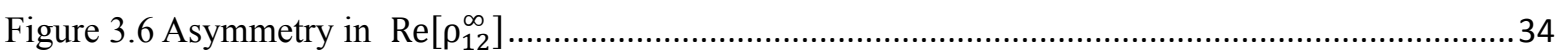

Figure 3.7 Rectilinear, diagonal, and circular components of Stokes vector ............................................. 35

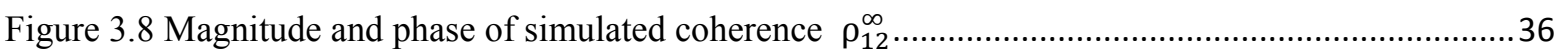

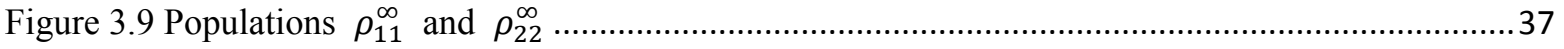

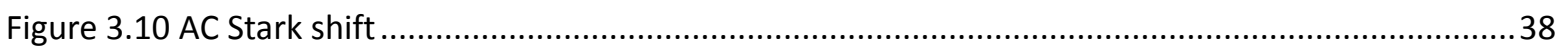

Figure 3.11 Simulated emission spectra from a 3-level system .............................................................. 42

Figure 3.12 Simulated $g^{(2)}(\tau)$ from a 3-level system .........................................................................44

Figure 4.1 QD's lifetime purturbed by above-band excitation laser .......................................................50

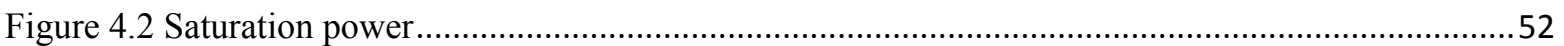

Figure 4.3 QD's RPLE spectra purturbed by above-band excitation laser ................................................53

Figure 4.4 Discete jumps in RPLE spectra from other QDs ................................................................54

Figure 4.5 Position, amplitude, and width of the Voigt peaks extracted from fittings ...............................55

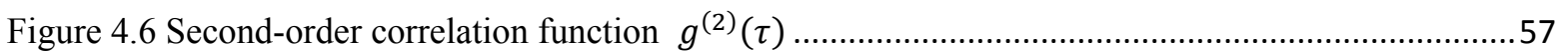

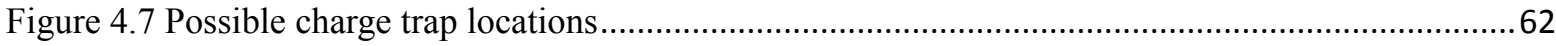

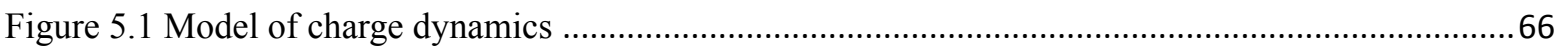

Figure 5.2 Schematic for AOM modulated experiment ......................................................................67

Figure 5.3 Schematic for heoterodyne measurement for characterizing coherent scattering.....................68

Figure A4.1 Model for QD's charge evolution ....................................................................................95 


\section{Chapter 1: Introduction}

In the past 20 years, semiconductor quantum dots have drawn a significant amount of research attention due to their rich physics and high potential for photonics and quantum information applications. The strong interaction of an optically active QD with its local environment establishes several new physics scenarios, such as the strong coupling of a single QD with a photonic cavity [7,19], the direct exchange interaction of a QD spin with the nuclear spin reservoir [20,21], and quantum coupling in a quantum dot molecule [22-24]. Meanwhile, the grail of quantum information and quantum computing drives researchers to study QDs as a promising qubit candidate with great effort. Spectacular achievements have been made in the field within the past few years, including the coherent optical control of a single electron spin in a QD [25], the entanglement of distant QD spins through a spin-photon interface [26], the photonic quantum logic gate controlled by a QD strongly coupled to a nanocavity [27], and single photon switching on ultrafast timescales [28].

Many different types of semiconductor QDs have been developed [29], such as CdSe colloidal QDs synthesized in chemical solution [30], the monolayer thickness fluctuations in a GaAs quantum-well [31,32], the electrostatically defined QDs in a two dimensional electron gas [33,34], and epitaxially grown self-assembled QDs $[35,36]$ like InGaAs. The latter possesses the advantages of a large carrier confinement, tailorable dot size in ensemble [37-39] and engineerable composition [40]. It also provides the best means for incorporation into complex structures for various electronic and photonic applications. These advantages are highly appreciated by the industrial community for making practical solid-state devices with these QDs, such as electrically pumped QD lasers [41] and infrared single photon detectors [42-44], some of which are already in the industrial production line.

This dissertation concerns self-assembled InGaAs QDs grown by molecular beam epitaxy (MBE). Although self-assembled QDs have proved to be excellent single photon sources with high brightness, narrow bandwidth and good spin-photon interface $[1,2,45]$, challenges still remain for practically using them to produce flying qubits for quantum communication and linear optical quantum computing. One difficulty is the scalable deterministic positioning and spectral matching of the QDs with respect to the device structure. This lack prevents efficient addressing of each qubit on-demand and impairs the quantum entanglement between the qubits via reducing the indistinguishability between the photons emitted by different QDs. The other issue is the relatively short spin coherence time $\mathrm{T}_{2}^{*}$, which constrains the number of operations one can implement before the electron spin gets kicked into a "random" and unknown direction [46]. This dephasing process happens mainly through two mechanisms: 1) magnetic environment fluctuations due to the nuclear spin reservoir; 2) electric environment fluctuations due to charge traps.

For the InGaAs QD system, none of its component elements possess zero nuclear spin, leading to a complicated exchange interaction between the QD spin and each nuclear spin. The number of nuclear spins within the wave function of the confined charges is on the order of $\sim 10^{5}$. Conventionally, these interactions are described by an effective magnetic field, called the Overhauser field, as an ensemble average of all nuclear magnetic moments. The random fluctuations of the nuclear spins cause the stochastic evolution of this effective magnetic field over time, resulting in the fast dephasing of the QD spin. Polarizing nuclear spin in a strong magnetic field helps 
suppress this dephasing effect, but so far only a maximum of $\sim 50 \%$ nuclear spin polarization has been achieved [47]. Nevertheless, it is possible to extend the coherence time to micro-second regime by employing optical techniques, such as nuclear spin locking via coherent population trapping [48,49] or through a "dragging" effect [50-52] in which the nuclear spins are aligned via dynamical nuclear polarization (DNP).

Comparatively, the second electric mechanism happens at a slower rate, typically on the order of kilohertz, which is quite slow compared to the first mechanism, and it cannot be the limiting factor on the dephasing. However, the energy levels of the QDs are shifted through the dc Stark effect when the local electric field changes, which is called spectral diffusion [53-55]. This mechanism impairs or destroys the indistinguishability between the photons emitted by the same QD at different times. This is troublesome since only indistinguishable photons (with identical wavelength, bandwidth, polarization and spatial profile) demonstrate Hong-Ou-Mandel interference [56], which underlies the basic entangling mechanism in linear optical quantum computing [57]. Possible mechanisms for the electric environment fluctuations are the capture or release of charge carriers from the local defect states assisted by phonons [58,59] or via Auger-processes [60]. Further studies show that these charge dynamics still exist even under strictly resonant excitation of single QDs [55,60,61]. Moreover, the quenching of the QD fluorescence under resonant excitation [62] imposes an extra challenge for practical use of these dots in various applications. The exact physical process of charge relaxation into a dot is still an open question, and the role played by the wetting layer or the continuum states is not clear [63-65]. Characterizing and understanding these charge dynamics (for both QD and defects) is a critical step for contriving the future suppression of these effects. Chapter 4 of this dissertation investigates the effect of the local charge environment on resonance fluorescence of a single QD by implementing resonant photoluminescence excitation (RPLE) spectroscopy and second-order correlation measurements at different levels of free charge carrier density introduced by an above band-gap laser. RPLE spectra indicate that the QD experiences discrete spectral shifts and continuous drift due to changes in the local charge environment. Statistically, these effects are shown as photon bunching under resonant excitation. But for purely above-band excitation, no bunching is observed, implying that there are two pathways for a QD to evolve to the exciton state, i.e., either by directly creating an electron-hole pair by absorbing a resonant photon, or by capturing free charge carriers introduced by above-band excitation at local environment. The competition between these two processes and the dynamics of the nearby charge traps result in the strong bunching observed in the correlations.

The other way to get around the spectral diffusion is to use coherent scattering with resonant excitation. For the past decade, many resonant fluorescence (RF) experiments have been carried out on these semiconductor QDs [66-70], and the different roles played by coherent and incoherent scattering have been investigated $[18,71,72]$. Specifically, when a QD is excited in the small Rabi frequency limit - the Heitler regime $[18,73]$ - the scattered photons are phase-locked to the excitation field, and exhibit a subnatural linewidth with long coherence time [18,72]. Moreover, these photons display a good single-photon purity, which provides an immediate opportunity for generating tunable and time-synchronized single photons from multiple QDs with near-arbitrarily tailored spectral and phase properties [18]. However, the coherent scattering intensity of a 2level system degrades when $\Omega_{\mathrm{R}}>0.125 \Gamma_{\mathrm{sp}}$, where $\Omega_{\mathrm{R}}$ is the Rabi frequency and $\Gamma_{\mathrm{sp}}$ is the population spontaneous decay rate. Moreover, the coherent scattering purity experiences a monotonic decrease as the excitation power increases. So is there a system that can generate coherently scattered single photons with high brightness and high purity? The answer is yes. In Chapter 3 of this dissertation, we propose that a 3-level V- 
shaped system, a neutral quantum dot, is potentially capable of achieving this goal. We show that comparing with a single 2-level system, a 3-level system can simultaneously reach $90 \%$ coherent scattering purity while maintaining coherent scattering intensity at a level equivalent to the maximum that a single 2-level system can practically produce. Together with the fact that a 3-level system generally possesses a broader spectrum than 2level system, this new quantum emitter is probably a better single photon source with broader scattering bandwidth, thus capable of generating single photons with near-arbitrary waveforms via shaping the excitation laser field [74]. Due to the presence of the coherent scattering, we predict that the excitation spectra of the neutral QD will show an unconventional line shape depending on both excitation and detection polarization, which we have confirmed experimentally. Within the framework of the semi-classical theory, we are able to reproduce the experimental results and summarize three relatively unrestrictive conditions for observing these peculiar line shapes as 1) a pair of orthogonal dipole moments, 2) arbitrary excitation and detection polarizations, and 3) relatively small splitting between two lines. Thus, similar effects are possibly present in other systems, such as a charged QD in an in-plane magnetic field [46], NV centers in diamond [75], and defect-bound states in 2-D materials [76].

In this dissertation, I will give a brief introduction on the fundamentals of self-assembled quantum dots in Chapter 2, including the origin of this 3D confined nanostructure, its electronic and optical properties, and the optical techniques used for measuring these properties. Chapter 3 will focus on investigating an unconventional excitation line-shape from a neutral QD, and we propose that a 3-level system is a better single-photon source compared to a single 2-level system in terms of coherent scattering performance. Chapter 4 studies how electric environment fluctuation affects a QD's emission properties. I will show that the excitation spectra of the QD provide location information about the fluctuation source and the second-order correlation measurements capture the time-scale of the dynamics. Then I will conclude my dissertation in Chapter 5 with an outlook to several future projects. 


\section{Chapter 2: Background}

Summary. In this chapter, we introduce the concept of quantum dot (QD) by demonstrating its origin in physical vapor deposition (PVD) and its optical properties under different conditions. The first section discusses the growth of the self-assembled InGaAs quantum dots by the means of molecular beam epitaxy. The second section focuses on the QD's energy structure and its optical properties within or without magnetic field. The last section introduces two powerful optical techniques for characterizing these optical transitions: polarizationdependent resonant photoluminescence excitation (RPLE) spectroscopy and time-tagged photon-correlation measurement. 


\subsection{Growth of Self-assembled Quantum Dots}

The state-of-art crystal growth techniques are relied on the concept of epitaxy, where the deposited atoms forms a crystalline structure that matches that of the underneath substrate. When the lattice constant (bulk value) of the deposited material does not match that of the substrate, the epitaxial growth cannot sustain and one consequence is the formation of the QDs. For InAs/GaAs system, the larger unit cell of the InAs (bulk) is compressed to accommodate the smaller lattice constant (7\% smaller) of the GaAs. This tetragonal deformation of the InAs lattice introduces an extra elastic energy to the system and it builds up quickly as the film gets thicker. When the InAs reaches a critical thickness of about 1.6 nominal monolayer (ML) [77-79], this strain energy becomes too high for the layer-by-layer growth mode to continuing and the nucleation of three-dimensional islands is
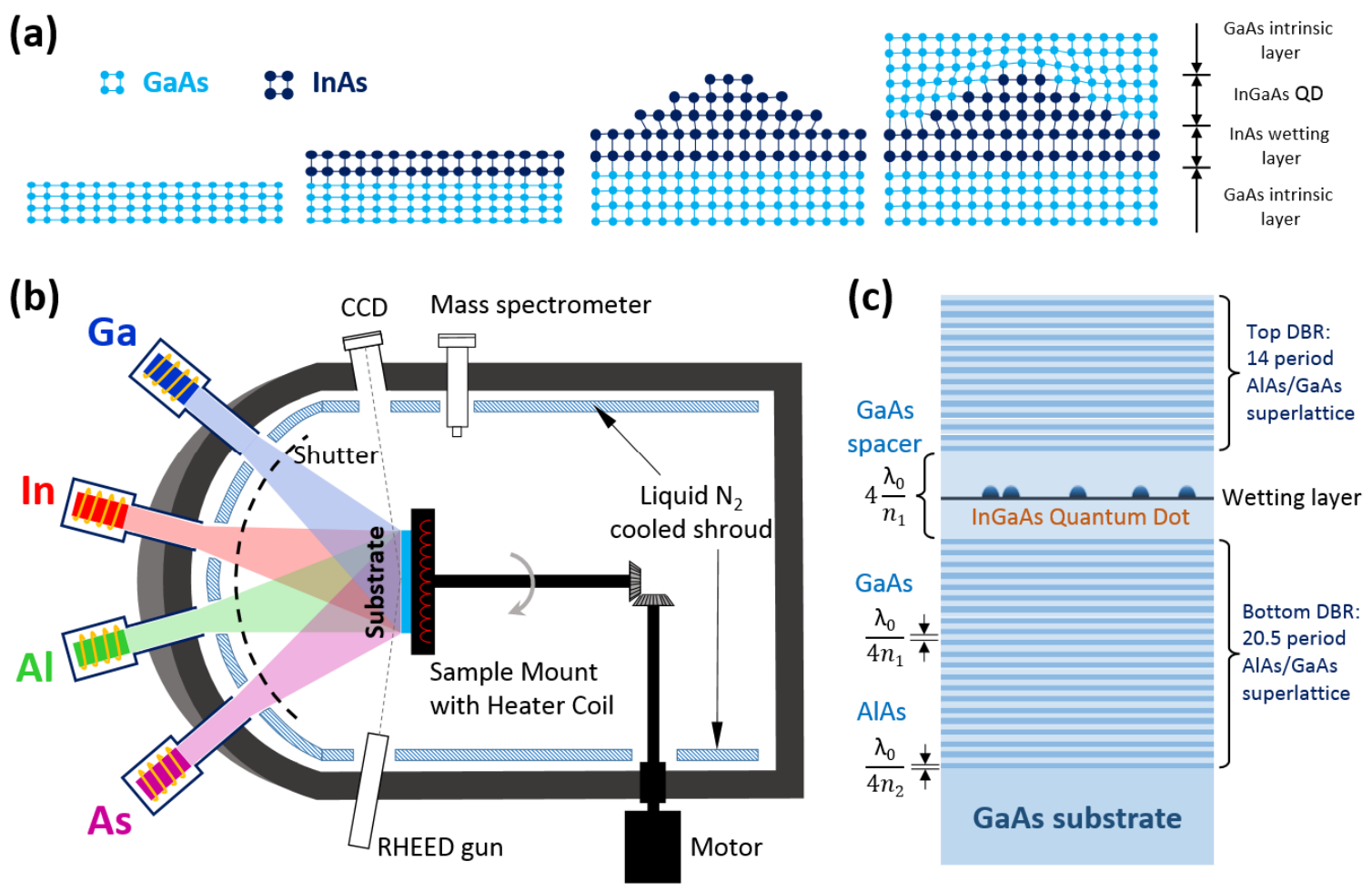

Figure 2.1 (a) Main steps to obtain self-assembled InGaAs QDs via Stranski-Krastanov mode of epitaxial growth. From left to right: Intrinsic GaAs layer, formation of wetting layer, nucleation (2D to 3D transition), and capping of the QDs. (b) MBE chamber for growing InGaAs QDs. The high-purity group-III and group-V materials are sealed and heated up in the effusion cells (K-cell) to supply the constant atomic flows for epitaxy growth. The liquid-nitrogen cooled shrouds condense the unwanted evaporants and improve the vacuum status around the sample. The whole environment is monitored by the mass spectrometer. RHEED provides the realtime monitoring of the morphology of the sample surface during the deposition. (c) Structure of a grown sample. The InGaAs QDs are embedded at the middle of a GaAs spacer which is bounded by two DBR superlattices. The thicknesses of all layers are in scale. $\lambda_{0}=930 \mathrm{~nm}$ corresponds to the center wavelength of the fluorescence of the QD ensemble in the sample. $\mathrm{n}_{1}=\mathrm{n}_{\mathrm{GaAs}}=3.45$ and $\mathrm{n}_{2}=\mathrm{n}_{\mathrm{AlAs}}=2.98$ are the refractive index of GaAs and AlAs at $\lambda_{0}$, respectively [89-92]. 
triggered to relieve the extra energy. The formation of coherently strained islands on the top of a flat film (the wetting layer) is conventionally known as Stranski-Krastanov (S-K) mode $[80,81]$ of heteroepitaxial growth or layer-plus-island mode. These islands are transformed into QDs by embedding them into a larger band gap material, e.g., InAs islands in GaAs. Figure 2.1(a) illustrates the main steps of forming self-assembled QDs. In reality, this is a fairly complicated process involving more dynamics than those shown in this schematic, such as the In-Ga intermixing in QDs, the In concentration gradient across the QDs $[82,83]$, and the erosion of the step edge surrounding QDs [84]. Due to the presence of intermixing of element Ga in the QD, these QDs are conventionally referred as InGaAs QDs. Via various morphology measurement techniques, such as AFM [78], TEM [85], STM [86] and RHEED [87], deeper understanding on the formation of these QDs has been reached, but the topic is out of scope considered by this dissertation and one can find a comprehensive review on the topic in references [88] and [35].

In various physical vapor deposition techniques, molecular beam epitaxy (MBE) is probably the best for obtaining the high-quality layer-by-layer grown thin film. The low deposition rate of MBE usually allows the films to grow epitaxially. The thickness variation between two repeated MBE growths is typically within \pm 0.05 $\mathrm{ML}$, due to the inevitable fluctuations in growth environment (such as beam fluxes, substrate temperature or vacuum status). Figure 2.1(b) shows an MBE chamber dedicated to grow InGaAs QDs. In ultra-high vacuum (UHV) (10-10 10-11 torr) environment, a 001-oriented GaAs substrate is held at $550 \mathrm{~K}$ and impinged by the atomic beams evaporated from the heated Knudsen cells (K-cell). The chemical composition or the stoichiometry of the depositing film can be controlled by adjusting the aperture on the shutter or the temperature of the K-cells.
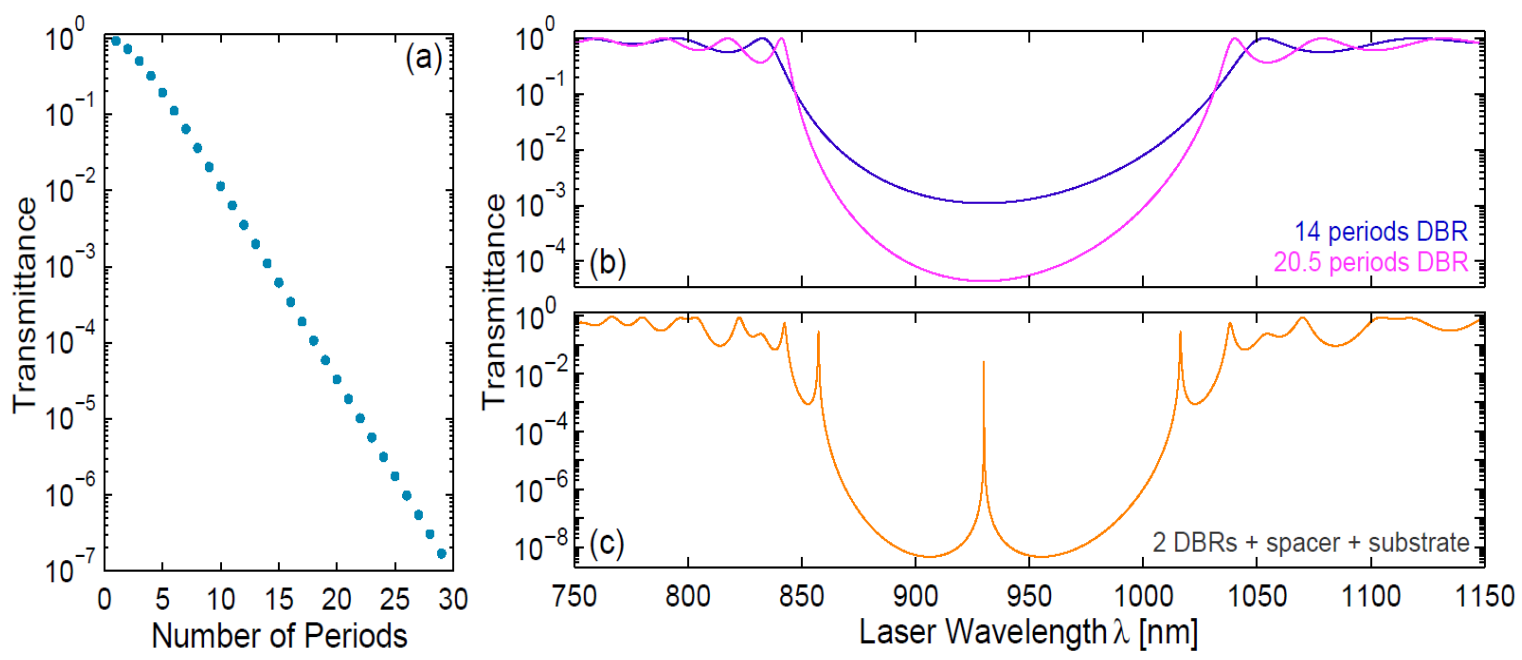

Figure 2.2 (a) Transmission (in logarithmic scale) of a single DBR mirror calculated at resonance wavelength (930 $\mathrm{nm}$ in vacuum) as varying the number sub-layers in the superlattice. Each period consists of one GaAs layer and one AlAs layer with thicknesses corresponding to one quarter of the resonance wavelength in each medium. (b) Wavelength-dependent transmission (in logarithmic scale) of a single DBR with 14 or 20.5 periods of sub-layers, corresponding to the top and bottom DBR mirror used in our sample. The DBR is bounded by air. The calculation does not consider the refractive index variation at different wavelength, which should be small. (c) Transmission (in logarithmic scale) of the whole sample structure at different wavelength. The calculation includes both DBR mirrors, the GaAs spacer, and the GaAs substrate. A transmission peak is shown at $\lambda_{0}=930 \mathrm{~nm}$. 
Rotating the substrate during the growth help to remove the angular dependence of K-cell flux and improves the thickness uniformity across the sample. The UHV provides low background contamination and allows for insitu real-time monitoring of the growth via reflection high energy electron diffraction (RHEED), which also enables on-the-fly study of the surface morphology evolution during the deposition.

In our sample, the InGaAs QDs are embedded in a GaAs spacer bounded by two distributed Bragg reflectors (DBR) as shown in Figure 2.1(c). Each DBR possesses a superlattice structure where the alternating GaAs and AlAs sublayer is designed to have a thickness of $\lambda_{0} / 4 \mathrm{n}_{\text {GaAs }}$ and $\lambda_{0} / 4 \mathrm{n}_{\text {AlAs }}$, respectively. Wavelength $\lambda_{0}$ is chosen to be the center of the fluorescence spectrum of the QD ensemble in the sample, for our case $\lambda_{0}=930 \mathrm{~nm}$. With this thickness, the many reflections from the superlattice for a wave at $\lambda_{0}$ interfere constructively, and the DBR acts as a high-quality reflector. The resultant reflectivity is strongly dependent on the number of sublayers contained in the structure, as shown by the simulation in Figure 2.2(a), calculated in matrix formulism [93,94]. Note that Figure 2.2 choose to show transmission result rather than reflectivity, since transmission can be better visualized in logarithmic scale. One can always infer the reflectivity by using normalization condition: transmission plus reflection equals unit. We choose 20.5 periods for bottom DBR, giving a reflectivity of $99.7 \%$ (at $\lambda_{0}$ ), which is slightly higher than $97.4 \%$ reflectivity exhibited by the top mirror with 14 periods. This encourages the extraction of the fluorescence from the top of the sample. The extraction efficiency is estimated to be $6 \%$ for a sample with similar planar cavity structure [14]. Figure 2.2(b) shows the calculated wavelengthdependence of the transmission of these two DBRs used in our sample. Within the wavelength range of 880 980 $\mathrm{nm}$, these mirrors are highly reflective and form a planar waveguide, enabling the light to propagate along the plane of the GaAs spacer.

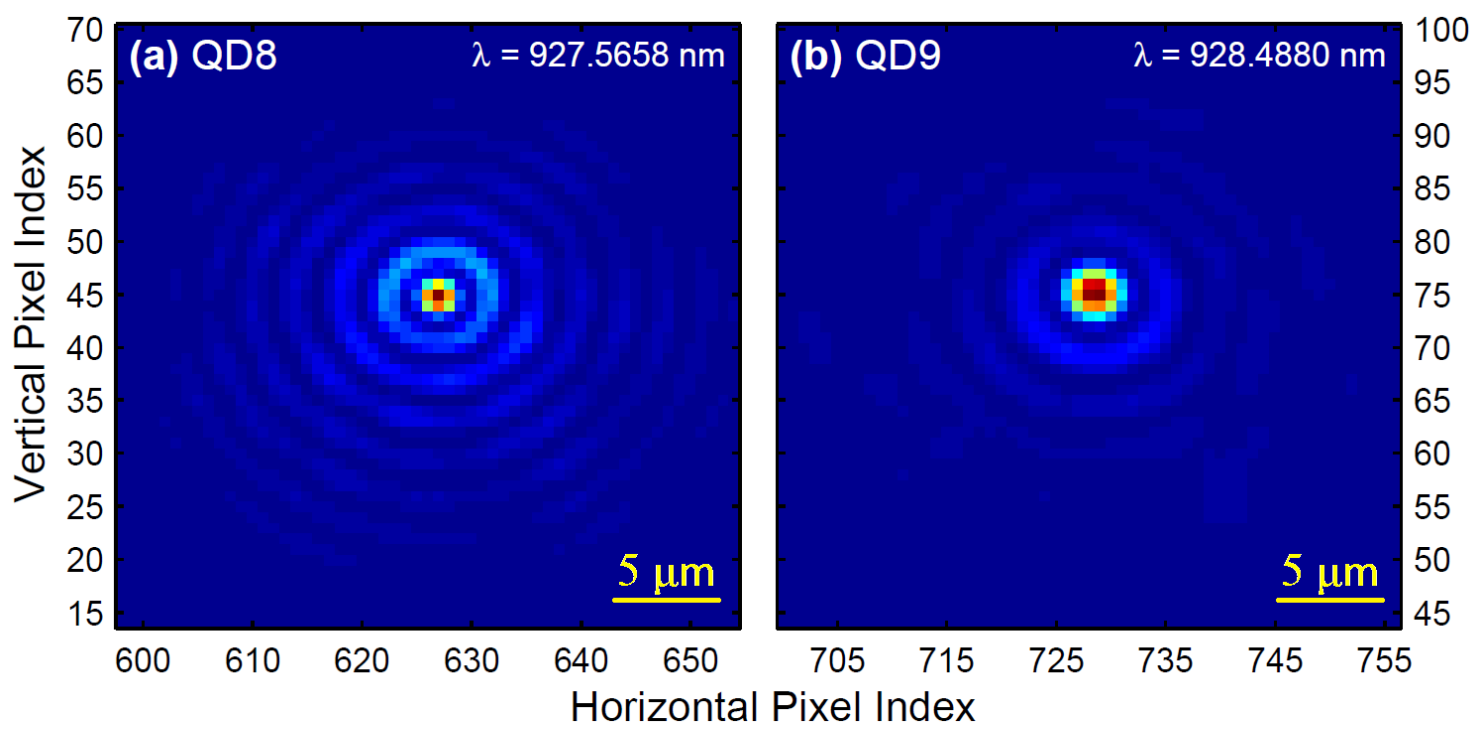

Figure 2.3 The spatial image of the other two QDs under resonant excitation. Both images are taken with 40x magnification, which results in an equivalent length of $0.5 \mu \mathrm{m}$ on sample for each pixel shown in the figure. QD8, resonance at $927.5658 \mathrm{~nm}$ in (a), shows a smaller center disk accompanied by a denser ring structure, comparing to QD9 in (b), resonance at a longer wavelength of $928.4880 \mathrm{~nm}$ with wider-spread rings. 
The transmission of the whole sample for normal incidence, however, shows a narrow dip at center wavelength $\lambda_{0}$, as shown in Figure 2.2(c). This is known as the Fabry-Perot mode of the cavity, since it shares the same underlying mechanism as employed by a Fabry-Perot interferometer. For our case, only the light field at wavelength $\lambda_{0}$ experiences a constructive interference in the cavity, resulting in a significantly enhanced light field that enables a measurable transmission through the high-reflectivity DBR mirrors. This provides the transmission mode for coupling the light out of the structure. The thickness of the GaAs spacer is chosen to be $4 \lambda_{0} / \mathrm{n}_{\mathrm{GaAs}}$, so that an anti-node of the cavity mode is formed at the middle the spacer where the QDs are located. This enhances the dot-field interaction, and causes more photons to be scattered into this output mode. For the photons at $\lambda_{0}$, the Fabry-Perot mode is along the surface-normal direction. But many QDs emit at a wavelength slightly off from $\lambda_{0}$ due to dot size variations. For these photons, the cavity mode is no longer perpendicular to the sample surface, but tilted to an angle depending on the wavelength. Only the photons scattered into this hollow cone can be coupled out, forming an output beam with ring-shape cross-section profile. Specifically, shorter wavelength corresponds to a transmission cone spanned with a larger apex angle, i.e., larger numerical aperture, which lead to a tighter focus in imaging system [95]. This argument is supported by the fact that the QD with shorter emission wavelength always manifests a smaller center disk and tighter ring structure in its image, as shown in Figure 2.3. Calculation of the output mode of a planar cavity also confirms the angled output when the wavelength is shorter than $\lambda_{0}$ [96]. Due to the imaging, the ring-shape intensity profile of a QD (a point source couples out of the cavity) cannot be seen in Figure 2.3. Instead, the image reflects a Fourier-transform of such ring structure.

Most experimental data shown in this dissertation are collected from two QDs found on the same piece of sample. For clarity and easier reference, we label them as QD1 and QD2, respectively. QD1 is investigated in Chapter 3 to study the unconventional excitation line shape observed for various detection polarizations, while QD2 is investigated in Chapter 4 that focuses on how the QD's photoluminescence property is affected by the fluctuation of local electric environment. Any data collected from other QDs will be explicitly pointed out for clarity. 


\subsection{Energy Structure \& Optical Properties of a Single Quantum Dot}

The atom-like wavefunction of each confined particle and the resultant discrete optical lines impart these semiconductor nanostructures as "artificial atoms", for the similarity to the atoms. Each "artificial atom", quantum dot, contains as many as $10^{4}$ atoms, which create the confining potential for the trapped charge carriers. The tunable absorption and emission wavelengths (via simply varying the nanocrystal size) make these quantum dots as a model system for studying the interaction between light and charge carriers in matter, in a much more flexible and feasible way than for atoms. In this section, we will explore the origin of these discrete energy structure and discuss the resultant optical properties for different circumstances.

\subsubsection{Single particle states}

The discrete energy structure of a self-assembled InGaAs QD originates from the type-I band alignment between the embedded and surrounding materials, which enables the three-dimensional confinement of a charge carrier in a small volume. The de Broglie wavelength of a free charge carrier in bulk semiconductor is approximately on the order of $\lambda=2 \pi \hbar / \sqrt{2 m^{\star} E}$ where $m^{\star}$ is the effective mass of the carrier, and $E$ is the kinetic energy that can be estimated by $(3 / 2) \mathrm{k}_{\mathrm{B}} \mathrm{T}$. At $4 \mathrm{~K}$, an electron in conduction band of GaAs or InAs has a wavelength of 340 or $200 \mathrm{~nm}$, while for a heavy hole, it is 80 or $70 \mathrm{~nm}$. In comparison, the size of the confinement potential is approximately $20 \mathrm{~nm}$ in lateral plane and $5 \mathrm{~nm}$ in vertical direction, which are significantly smaller than the de Broglie wavelength of free charge carriers. Therefore, strong confinement regime for both electron and hole are achieved, which results in an atom-like discrete energy structure as shown in Figure 2.4(a).

The number of the discrete energy levels formed in the potential well depends on the depth of the potential and its base length. The depth is almost solely determined by the band gap of the materials, which are basically fixed when the materials are selected. However, the size and shape of the dots can be controlled statistically via tuning the growth parameters. Generally, a smaller size the dot is, the higher the energy levels are. Figure 2.4(b) depicts the QD's size dependence of these energy levels, calculated by Schliwa et al. [97] by modeling the QD with 8band $\mathbf{k} \cdot \mathbf{p}$ theory $[98,99]$. It is clear that the energy levels of electron and hole shift towards the respective band edges of the surrounding medium (GaAs) when the size of the dot is reduced. This energy shift leads to a reduction of the number of bound states with decreasing dot size. In this particular example, there are only 3 electron bound states and 5 heavy hole bound states at a base length of $10 \mathrm{~nm}$, whereas these numbers increase up to more than 12 for both charge species at a base length of $20 \mathrm{~nm}$.

More theoretical simulations $[98,100-102]$ indicate that if one wants to calculate the single particle energies to an accuracy required by the experiment, many other factors need to be considered, such as detailed physical shape of the QD, charge redistribution due to piezo-electric effect, and most importantly, the strain within the QD. For example, the indium-rich apex of the QD tends to introduce a large uniaxial strain locally that has a powerful confining effect on the holes while almost no influence on the electrons. This leads to the confinement of the hole wave function on the top of the dot, above the localization of the electron wave function at the 

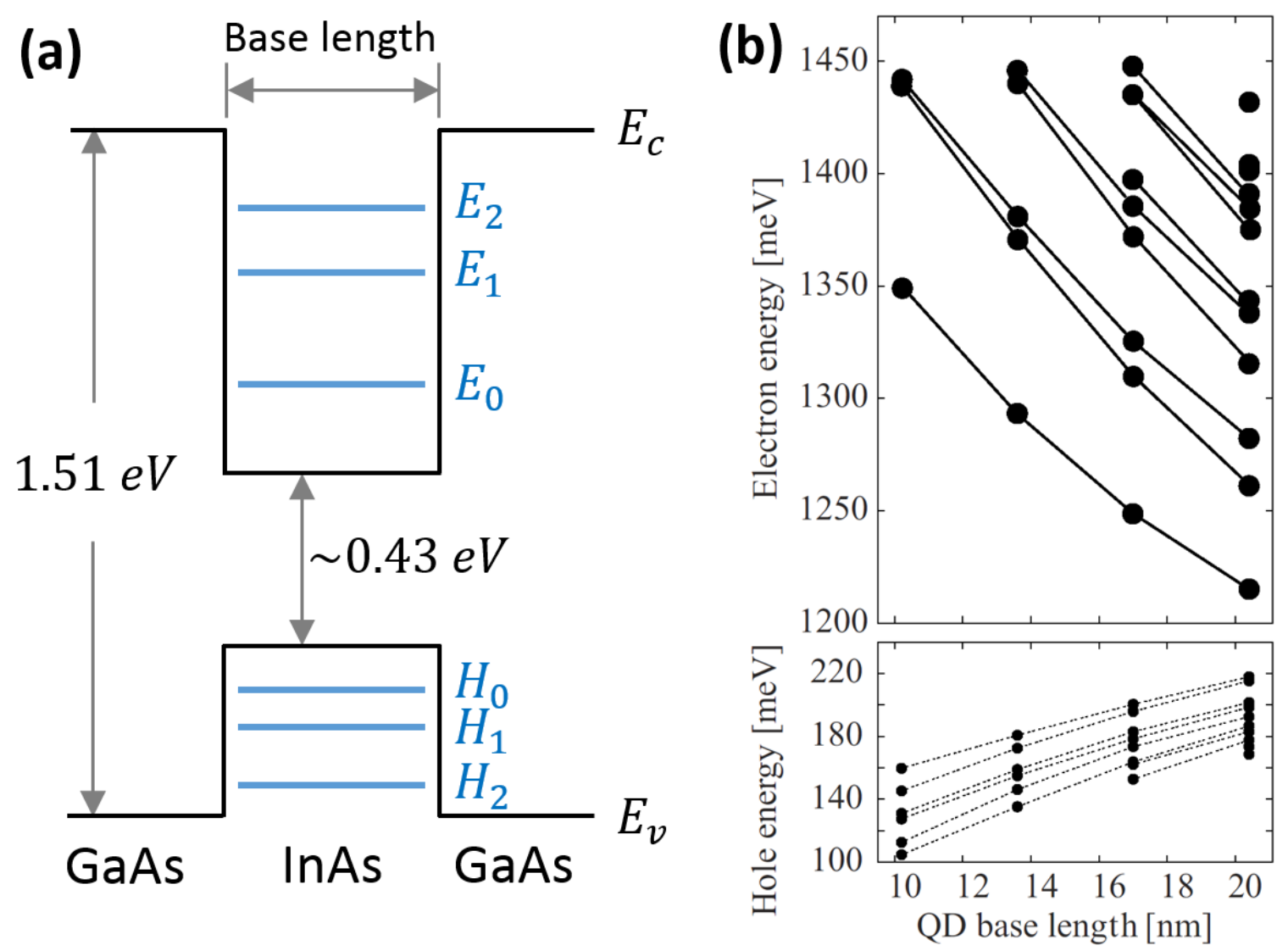

Figure 2.4 (a) Schematic of the single-particle states for the electron and hole confined in an InGaAs QD. The band-gap energies are given at $4 \mathrm{~K}$. (b) Size-dependence of the single-particle states for a pyramidal shaped QD, calculated by Schliwa et al. [97]. The calculation includes both the first and second order piezoelectric effect.

base [100]. Traditionally, this corresponds to a positive dipole moment (same direction as the growth axis). Conversely, calculations assuming pure InAs dots predict negative dipole moment, with the hole confined at the base of the dot below the electron wave function [99]. Experimentally, the post-annealed samples (annealing blue shifts the optical transitions to from $\sim 1200 \mathrm{~nm}$ to $900 \sim 1000 \mathrm{~nm}$ ) confirm the negative sign, implying an indium migration and a dissolution of the apex during the annealing process [103].

In addition, the strain reduces the symmetry of the crystal and removes the degeneracy between heavy-hole (HH) and light-hole (LH) states within the QD [104,105]. The predicted splitting is at least several tens of meV, and it can be as large as $0.5 \mathrm{eV}$ according to a 40-band calculation by Saidi et al. [102]. At cryogenic temperature, this energy difference is too big for thermal promotion, thus considerably reduces the possible admixture between HH and LH states. Consequently, the higher energy light-hole state can be safely neglected. 


\subsubsection{Optical properties}

The optical properties of a QD are mostly determined by the interaction between the electrons and holes bound in the lowest energy levels, i.e., $\mathrm{E}_{0}$ or $\mathrm{H}_{0}$ in Figure 2.4(a). The charge carriers in higher energy levels cannot recombine efficiently due to the presence of fast non-radiative decay process in the solid-state system (in 1 10 ps) [106], such as phonons. They would firstly decay to the lowest energy levels before the radiative recombination can take place in a time period of sub to 1 ns. Two kinds of interactions between electron and hole are mainly involved: Coulomb interaction between charges and exchange interaction between spins. The Coulomb attraction between electron and hole bounds the two charges together to form a quasi-particle called neutral exciton $\left(\mathrm{X}^{0}\right)$. The spin properties of these charge carriers determine the degeneracy of these exciton states. The energy of the exciton state is reflected by the emission wavelength of the QD, and the spin information of the exciton is imprinted in the emission polarization.

Since both electron and heavy-hole are doubly degenerate $\left(S_{e, \mathrm{z}}= \pm \hbar / 2\right.$ and $\left.J_{h . \mathrm{z}}= \pm 3 \hbar / 2\right)$, the resulting neutral exciton state is thus 4-fold degenerate, characterized by the four projections of the total angular momentum along $\hat{\mathrm{z}}$-axis, i.e., $M=\left(S_{e, \mathrm{z}}+J_{h, \mathrm{z}}\right) / \hbar$, where $\hat{\mathrm{z}}$ is the sample surface normal. In Dirac notation, we write electron spin with a thin arrow (e.g., $|\uparrow\rangle$ ), and heavy-hole spin with a thick arrow (e.g., $|\Uparrow\rangle)$. States with $|M|=$ $2,|\uparrow \uparrow\rangle_{\mathrm{z}}$ or $|\downarrow \Downarrow\rangle_{\mathrm{z}}$, cannot couple to the light field through electric dipole interaction, and are thus called dark excitons; while states with $|M|=1,|\downarrow \Uparrow\rangle_{\mathrm{z}}$ or $|\uparrow \Downarrow\rangle_{\mathrm{z}}$, are optically active, and are called bright excitons. The degeneracy between the dark and bright exciton manifold is lifted by the exchange interaction (spin-spin interaction) [107-109] between the electron spin $\left(S_{e, i}\right)$ and the heavy-hole spin $\left(J_{h, i}\right)$

$$
H_{\text {Exchange }}=-\sum_{i=\mathrm{x}, \mathrm{y}, \mathrm{z}}\left(a_{i} J_{h, i} S_{e, i}+b_{i} J_{h, i}^{3} S_{e, i}\right)
$$

where $a$ and $b$ are spin-spin coupling constants. The splitting energy between the two manifolds is $\delta_{0}=$ $1.5\left(a_{\mathrm{z}}+2.25 b_{\mathrm{z}}\right)$, without cross mixture between the two manifolds [110]. Meanwhile, the double degeneracy of the exciton states in each manifold is removed by a splitting of $\delta_{1}=0.75\left(b_{\mathrm{x}}+b_{\mathrm{y}}\right)$ for dark exciton states and $\delta_{2}=0.75\left(b_{\mathrm{x}}-b_{\mathrm{y}}\right)$ for bright exciton states. These splittings are accompanied by a hybridization of the states within each individual manifold.

For a dot exhibiting in-plane rotational symmetry (group $D_{2 d}$ ), i.e., $b_{\mathrm{x}}=b_{\mathrm{y}}$, states $|\downarrow \Uparrow\rangle_{\mathrm{z}}$ and $|\uparrow \Downarrow\rangle_{\mathrm{z}}$ are still the eigenstates of $H_{\text {Exchange }}$ and degenerate, as shown in Figure 2.5(a). These bright exciton states are optically accessible through circular polarized light $\left(\sigma^{-}\right.$or $\left.\sigma^{+}\right)$. The two dark exciton states are non-degenerate and not shown in the plot.

When the dot's rotational symmetry is broken by a uniaxial deformation (e.g., by strain), the anisotropicity $\left(b_{\mathrm{x}} \neq b_{\mathrm{y}}\right)$ of the exchange interaction intermixes $|M|=1$ states, and splits the final mixed states by an energy of $\delta_{2}$. This splitting is referred as the fine structure splitting (FSS) and is on the order of 1 to $40 \mu \mathrm{eV}$ for InGaAs QDs. The intermixture between $|\downarrow \Uparrow\rangle_{\mathrm{z}}$ and $|\uparrow \Downarrow\rangle_{\mathrm{z}}$ are equal-ratio, leading to a pair of bright exciton states that can be expressed as $|\downarrow \Uparrow\rangle_{\mathrm{x}}$ and $|\uparrow \Downarrow\rangle_{\mathrm{y}}$ in $\mathrm{x}-\mathrm{y}$ plane, as shown in Figure 2.5(b). The corresponding transitions thus 


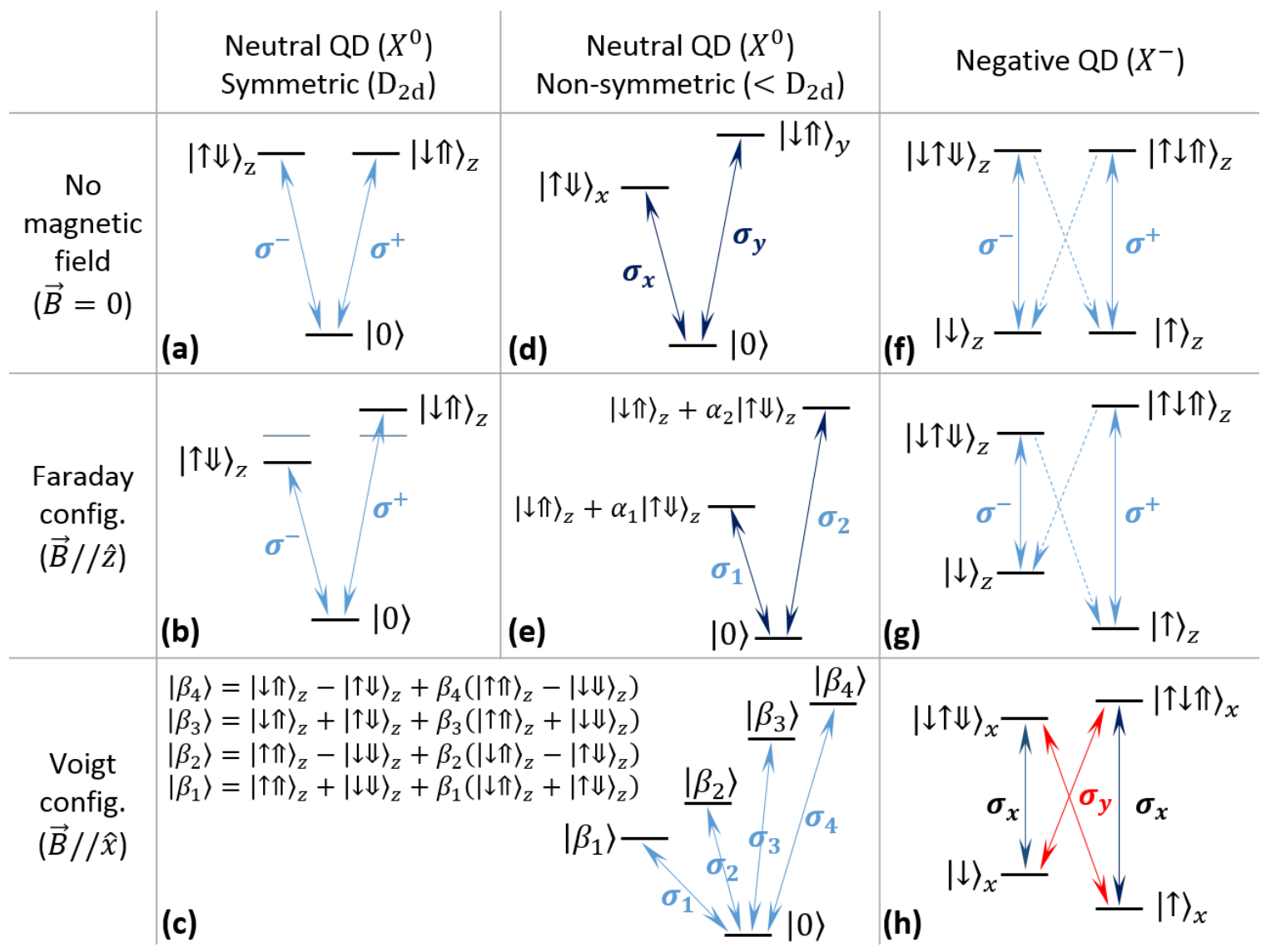

Figure 2.5 Optical properties of an InGaAs QD. In all cases, only the bright exciton states (optical-active) are shown. (a) Excitonic energy levels for a rotational invariant QD in zero magnetic field. The neutral exciton $\left(\mathrm{X}^{0}\right)$ state are doubly degenerate and coupled by left $\left(\sigma^{-}\right)$or right-circular $\left(\sigma^{+}\right)$polarized light, respectively. (b) Excitonic energy levels for a QD with broken rotational symmetry in zero magnetic field. The asymmetric exchange interaction between the electron and the hole leads to a new pair of eigenstates $|\uparrow \Downarrow\rangle_{\mathrm{x}}$ and $|\downarrow \uparrow\rangle_{\mathrm{y}}$, split by fine structure splitting (FSS). The two transitions become linear polarized. (c) Rotational-symmetric QD in Faraday magnetic field ( $\overrightarrow{\mathrm{B}} / / \hat{\mathrm{z}}$, with $\mathrm{z}$ pointing along the growth direction, or the surface normal of the sample). The Zeeman energy splits the two neutral exciton states. (d) Asymmetric QD in Faraday magnetic field $(\overrightarrow{\mathrm{B}} / / \hat{\mathrm{Z}})$. The out-of-plane magnetic field rotate the quantization axis out of $\mathrm{x}-\mathrm{y}$ plane, leading to new eigenstates. The coefficients $\alpha_{i}$ depends on the strength of the magnetic field and exchange energies. The splitting is larger than FSS. (e) In Voigt configuration $(\vec{B} \perp \hat{z})$, four optical-active lines are present for both symmetric and asymmetric case. The coefficients $\beta_{i}$ are determined by exchange energies $\left(\delta_{0}, \delta_{1}, \delta_{2}\right)$ and magnetic field $B$. The exact energy levels are given by reference [110]. (f) Excitonic structure for a negative trion $\left(\mathrm{X}^{-}\right)$in zero magnetic field. The two transitions are degenerate and circular polarized due to zero exchange interaction. (g) Negative trion $\left(\mathrm{X}^{-}\right)$in Faraday magnetic field $(\overrightarrow{\mathrm{B}} / / \hat{\mathrm{Z}})$. The Zeeman energy of each state simply lifts the degeneracy of two transitions. Cross transitions are not allowed due to the conservation of the angular momentum. (h) Negative trion $\left(\mathrm{X}^{-}\right)$in Voigt magnetic field $(\overrightarrow{\mathrm{B}} \perp \hat{\mathrm{z}})$. The in-plane magnetic field mixes the ground states $\left(|\uparrow\rangle_{\mathrm{z}}\right.$ and $\left.|\downarrow\rangle_{\mathrm{z}}\right)$ and the excitonic states $\left(|\uparrow \downarrow \uparrow\rangle_{\mathrm{z}}\right.$ and $\left.|\downarrow \uparrow \downarrow\rangle_{\mathrm{z}}\right)$, respectively, leading to four new linear polarized transitions. The two cross transitions are optical-active and in the same polarization. 
become linear-polarized. In MBE-grown samples, most QDs are non-circular shaped due to the stochastic property in assembling process for $\mathrm{QD}$, which means low probability to create a $\mathrm{QD}$ with rotational symmetry.

Even though no magnetic field is applied to any measurement in this dissertation, it worth a brief discussion here to show these energy structure in a magnetic field considering its broad interest for quantum applications. When an external magnetic field $\vec{B}$ present, the Hamiltonian is modified by the Zeeman energies of the electron and heavy-hole

$$
H_{\text {Zeeman }}(\vec{B})=H_{\mathrm{e}}+H_{h}=\mu_{B} \sum_{i=\mathrm{x}, \mathrm{y}, \mathrm{z}} g_{e, i} S_{e, i} B_{i}-2 \mu_{B} \sum_{i=\mathrm{x}, \mathrm{y}, \mathrm{z}}\left(\kappa_{i} J_{h, i}+q_{i} J_{h, i}^{3}\right) B_{i}
$$

where $\mu_{B}$ is the Bohr magneton, and $g_{e, i}, \kappa_{i}, q_{\mathrm{i}}$ are the (Luttinger-Kohn [111]) Zeeman splitting constants for the electron and hole $\left(\kappa_{i} \gg q_{\mathrm{i}}\right)$. The total Hamiltonian of the system thus becomes $H=H_{\text {Zeeman }}(\vec{B})+$ $H_{\text {Exchange}}$, which is complicated for a general magnetic field direction. Without losing generality, let us consider two simpler scenarios, with the external magnetic field $\vec{B}$ along the heterostructure growth direction (Faraday configuration), or in an in-plane direction (Voigt configuration).

In the Faraday case $(\vec{B} \| \hat{z})$, the Zeeman energy can be simplified by noticing $J_{h, z}^{2}=9 / 4$ for a heavy-hole

$$
H_{\mathrm{Zeeman}}^{\mathrm{F}}\left(B_{\mathrm{z}}\right)=\mu_{B}\left(g_{e, \mathrm{z}} S_{e, \mathrm{z}}-\frac{g_{h, \mathrm{z}}}{3} J_{h, \mathrm{z}}\right) B_{\mathrm{z}}
$$

where the effective heavy-hole $g$-factor $g_{h, \mathrm{z}}$ is $g_{h, \mathrm{z}}=5 \kappa_{\mathrm{z}}+13.5 q_{\mathrm{z}}$. For a dot with rotational symmetry $\left(D_{2 d}\right)$, the angular momentum eigenstates $|\uparrow \Uparrow\rangle_{\mathrm{z}}$ and $|\downarrow \Downarrow\rangle_{\mathrm{z}}$ are still the eigenstates of the total Hamiltonian, but the degeneracy between them is lifted by an energy splitting of $\Delta_{z}=\mu_{B}\left(g_{e, \mathrm{z}}+g_{h, \mathrm{z}}\right) B_{\mathrm{z}}$, as shown in Figure 2.5(c). For a QD with lower symmetry $\left(C_{2, v}\right.$ or $\left.C_{2}\right)$, the bright exciton states become the linear combination of the angular-momentum eigenstates and the mixing ratio is magnetic-field-dependent, as in Figure 2.5(d). The splitting deviates from a linear dependence to a form of $\Delta=\sqrt{\delta_{2}^{2}+\Delta_{Z}^{2}}$. For low magnetic field, it varies quadratically with $\mathrm{B}$, while for high field, it returns back to the linear dependence.

In the Voigt case (e.g., $\vec{B} \| \hat{x}$ ), the in-plane magnetic field destroys the rotational symmetry, and causes an admixture of bright and dark manifolds, resulting in the observable "dark" states in the spectra, as shown in Figure 2.5(e). Comparing with the Faraday configuration where an energy state crossing occurs between the low-energy bright exciton state and the high-energy dark exciton state as varying magnetic field, there is no states crossing between the hybridized four energy states in Voigt configuration. Reference [110] gives a good review on these energy structures.

Another common and important few particle state for self-assembled QDs is the trion. Two types of trion exist: negative trion $\left(\mathrm{X}^{-}\right)$and positive trion $\left(\mathrm{X}^{+}\right)$, composed of two electrons and one hole, or one electron and two holes, respectively. At the lowest energy, all carriers are in the ground levels, $\mathrm{E}_{0}$ or $\mathrm{H}_{0}$ in Figure 2.4(a). Thus, the two carriers with the same charge have to form a spin singlet to stay in the same level while the third carrier can have its spin in either up or down direction.

In zero magnetic field, the recombination energy of a neutral exciton $\left(\mathrm{X}^{0}\right)$ is usually higher than that of a negative trion $\left(\mathrm{X}^{-}\right)$, but lower than that of a positive trion $\left(\mathrm{X}^{+}\right)$[112]. This difference can be explained by using the 
binding energy in these three systems. Firstly, the final states (after recombination) of these three systems are energetically equivalent to each other because no interaction energy are involved for a single charge or empty state. Secondly, the negative trion can be visualized as adding a second electron to a neutral exciton, thereby two extra Coulomb interaction energies are added to the neutral system: repulsion between the two electrons $C(e, e)$, and attraction between the second electron and the hole $C(e, h)$. Similarly, for $\mathrm{X}^{+}$, the recombination energy is modified by $C(e, h)$ and $C(h, h)$. Due to the larger effective mass of the heavy-hole and the small dot size (strong confinement), the wave function of the hole is more localized than that of the electron [113]. For a crude approximation by considering a uniform distribution of charge in a sphere, the smaller radius of the distribution means larger potential energy. Consequently, $|C(e, e)|<|C(e, h)|<|C(h, h)|$, results in a positive binding energy for negative trions, and a negative binding energy for positive trions.

As for the optical properties of a trion, let us use the negative trion as an example. The arguments can be easily transferred to positive trions. The exchange interaction between each electron and the hole cancels each other, considering the opposite spin states possessed by two electrons when the system is in the lowest energy state. This cancellation is true at least for QDs in the strong confinement regime, where the wave functions of the two electrons have about the same spatial distributions, leading to a zero local spin density for the electrons [110]. Thus the two optically active exciton states are degenerate, as shown in Figure 2.5(f). The two final singleparticle states are also degenerate when no magnetic field is applied, leading to two circularly polarized transitions.

When an external magnetic field is applied, the degeneracies of both initial and final states are lifted. For initial trion states, the splitting is given by the Zeeman energy difference between the two heavy-hole spin states, while for the final states, the Zeeman energy of the electron spin state determines the splitting. In Faraday configuration, the four eigenstates $(|\uparrow \downarrow \uparrow\rangle$ and $|\downarrow \uparrow \Downarrow\rangle$ for initial; $|\uparrow\rangle$ and $|\downarrow\rangle$ for final) are simply shifted by the corresponding Zeeman energy, as shown in Figure 2.5(g). And no cross transition is allowed without the assistance of spin flipping processes [114]. In Voigt configuration, however, the in-plane magnetic field $(\overrightarrow{\mathrm{B}} / / \hat{x})$ rotates the quantization axis out of $\hat{z}$-direction, resulting in four new eigenstates that are superpositions of the original initial or final states. This lifts the restriction on cross transitions and four new transitions are formed. The two transitions with the lowest and highest energy are x-polarized, while the other two are y-polarized, as shown in Figure $2.5(\mathrm{~g})$. Once the exciton states are well separated, two $\Lambda$-systems are established: one exciton state $(|\uparrow \downarrow \uparrow\rangle$ or $|\downarrow \uparrow \downarrow\rangle)$ coupled optically with two single-electron ground states $(|\uparrow\rangle$ and $|\downarrow\rangle)$. This system is the cornerstone for coherent population trapping [115,116] and spin manipulation $[25,117,118]$. 


\subsection{Experimental Techniques and Setup}

Since the line width of an InGaAs QD $(\sim 1 \mathrm{GHz})$ is too narrow for a commercial grating $(1200 \mathrm{gr} / \mathrm{mm}$ gives a $\sim 35 \mathrm{GHz}$ resolution) to resolve, resonant photoluminescence excitation (RPLE) spectroscopy is adopted to investigate the energy structure of a QD. This technique measures the total fluorescence (spectrally-integrated PL) from the sample as the frequency of a continuous-wave $(\mathrm{cw})$ excitation laser is scanned across the QD resonance. The spectral resolution is solely limited by the bandwidth of the excitation laser, which can easily reach $1 \mathrm{MHz}$ regime [CTL manual] with the help of the external cavity diode laser (ECDL) [119-121]. For directly measuring the emission spectrum of a QD, a high-resolution scanning Fabry-Perot interferometer (FPI) is needed, which is under construction in our lab.

For temporal measurement, we use time-correlated single photon counting (TCSPC) to extract the time-sensitive information from the laser induced fluorescence via accurately timing the detection of individual photons. In practice, this is done by using a high-efficiency single photon detector to convert each photon event to a sharp electronic pulse, which is then time-tagged through a fast timing circuit at a resolution of 4 picoseconds. Finally, these arrival times are histogrammed statistically either with respect to the laser reference signals for the lifetime measurement, or with respect to other photon events for second-order correlation measurement, i.e., $g^{(2)}(\tau)$.

Figure 2.6 shows the optical setup to implement these measurements. The excitation laser (1) can be either a mode-hop free tunable cw laser (CTL from Toptica Photonics, AG) for RPLE and correlation experiments, or a pico-second pulsed laser (Mira from Coherent, Inc.) for lifetime measurement. Two lasers share the same optical path in our setup. The power of the excitation beam can be adjusted via neutral density filter (2) and monitored by a wave-meter (3) that picks $10 \%$ of the main beam with a non-polarized $10 / 90$ beam splitter (3). The transmitted laser is then coupled through a 2-meter long single-mode fiber (5) to select the fundamental spatial mode (Gaussian TEM $_{00}$ mode) for later excitation. This spatial mode can be focused more tightly thus reducing the laser scattering from sample's cleaved edge. The output from the fiber (5) firstly goes through a linear polarizer (6) to select Y-polarization (perpendicular to the table), and then a pair of confocal lenses (7) to adjust its beam waist to match the size of the aspheric lens (9) in the cryostat (8). This allows the tightest focusing of the excitation beam at the cleaved edge of the sample, thereby achieving the best coupling efficiency for introducing the excitation photons into the planar waveguide in the sample, as indicated by the zoom-in image of the sample. The QDs addressed by this excitation beam are located approximately $100 \mu \mathrm{m}$ from the cleaved sample edge. Most coupled photons would propagate through the GaAs spacer without experiencing any interaction, but a small proportion would "see" the scattering centers, such as QDs, wetting layer, or defect states, and experience an elastic or inelastic scattering. In free space, the scattered light can go any direction with equal probability, but in this sample, the existence of the planar cavity modifies this probability distribution in the favor of Fabry-Perot mode, i.e., more photons would be scattered into the directions close to surface normal. These photons are coupled out of the structure, collected and collimated by an aspheric lens (10) with a numerical aperture of 0.5 in the cryostat. 


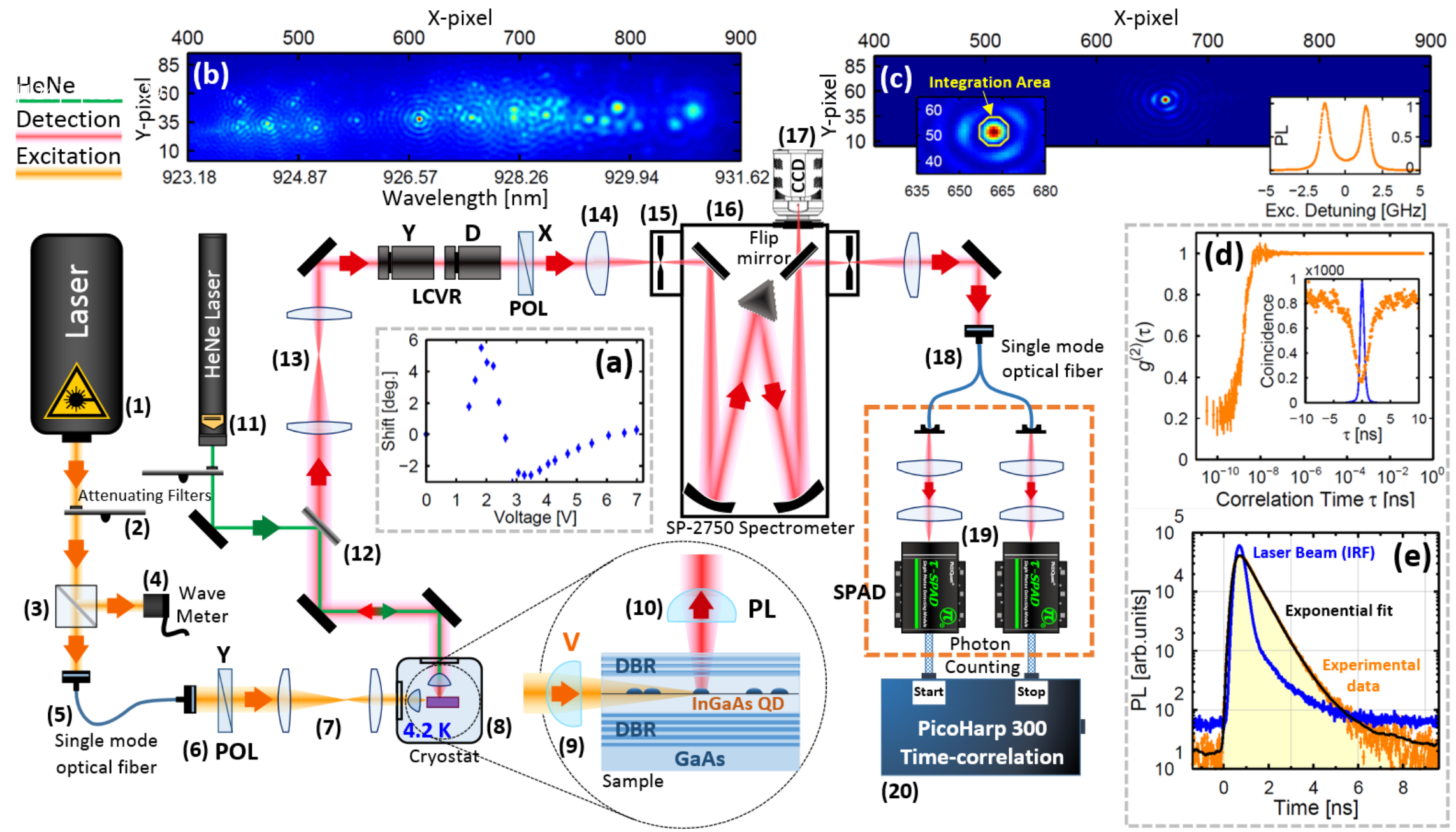

Figure 2.6 Optical setup for RPLE and correlation measurements. Details can be found in the text. (a) Measured fast-axis shift of the second LCVR (D) [124]. (b) Photoluminescence of the sample with just HeNe excitation. The frame was taken with the horizontal (X) detection polarization, open slit (17), and grating on. The exposure time is 10 sec. (c) Photoluminescence of the sample excited by a cw narrow-band laser (1), tuned to be resonance with QD1. The exposure time is 3 seconds. Left inset: a zoom-in view of the fluorescence of QD1. Right inset: An RPLE spectrum of QD1 by scanning the excitation frequency. (d) Measured second-order correlation function $g^{(2)}(\tau)$ of QD2 excited by HeNe laser. The raw data has been processed with a multiple-tau code [125-129] to show a logarithmic correlation time spanning 10 orders of magnitude. Inset: the same data but processed with a linear-scale code. Blue line is the measured instrument response of function (IRF) of two $\tau$-SPAD detectors. (e) Timeresolved fluorescence of QD1. Orange dots are the raw data. Black curve is an exponential fit that has convolved with the measured IRF (blue curve) of a single $\tau$-SPAD. 
The orthogonal geometry of collection and excitation path naturally discriminates the weak fluorescence signal from the strong excitation beam by taking advantage of their different propagation directions. An alternative way to do this is through a confocal dark-field technique where the polarization degree of freedom is used to separate the weak fluorescence from the strong excitation reflection because the two overlap both spatially and spectrally $[122,123]$. By using a pair of high-quality commercial linear polarizers, a suppression factor of $10^{7}$ on the scattered laser light has been achieved, leading to a background-free detection of resonance fluorescence [123]. This technique is preferred when the freedom of excitation polarization is needed, especially for circular polarizations. The trade-off of is the locking of the detection polarization with respect to the excitation polarization, which prohibits the polarization study of the fluorescence. In comparison, the sideexcitation geometry frees the detection polarization by trading off the freedom on excitation polarization. In Chapter 3, we will see that the freedom of detection polarization is critical for the spectral analysis, and is necessary for coherence extraction.

For above band-gap excitation, we use a HeNe laser (11) at $632 \mathrm{~nm}$. For this wavelength, the side excitation is no longer feasible because GaAs becomes strongly absorptive. Thus, we direct the HeNe beam to the sample through the PL collection path by using a dichroic mirror (12). The HeNe beam is depicted by green line in Figure 2.6. This $1 \mathrm{~mm}$-thick dichroic mirror (12) is strongly reflective (93\%) for HeNe light (630 805 $\mathrm{nm})$ while effectively transmissive (80\%) for the photoluminescence from the sample (880 1600 nm). The HeNe light reflected from the sample surface or cryostat window could mix with the PL, but it can be easily filtered out with grating in the spectrometer (16) (Acton 2750 by Princeton Instruments, Inc.) before being collected by a charge coupled device (CCD) (17).

To select a detection polarization, we use a pair of liquid crystal variable retarders (LCVRs) and a linear polarizer setting up in front of the spectrometer. These three optics together act like a "general" polarizer that can project the incident light onto any polarization axis on the Poincare sphere, including elliptical and circular polarizations. This is achieved by aligning the fast-axes of the two LCVRs to be 45-degree away from each other (in our case, they are vertical and diagonal), thereby forming a pair of perpendicular rotation-axis in the linear polarization plane of the Poincare sphere. Consequently, any point (polarization state) on the Poincare sphere can be reached via two consecutive rotations about these two axis, with the rotational angles determined by the voltage applied on each liquid crystal. However, the liquid crystal has an intrinsic deficiency: the voltage-dependent position of the fast-axis, or fast-axis shift. The shift is usually small [less than $5^{\circ}$, see Figure 2.6(a)] but unique to each device. This complicated dependence makes it difficult to compensate for it optically. This becomes one of main sources of uncertainty for our polarization analysis.

In the fluorescence collection path, we build a microscopic imaging system to search and pinpoint the single QDs for study. It consists of two parts: a 20x magnification objective constructed by the aspheric lens (10) in the cryostat and the achromatic doublet (14) in front of the spectrometer, and a 4x magnification Keplerian telescope constructed with two plano-convex lens (13). Therefore, a final 80x magnification on the fluorescence is achieved, which is recorded by a charge coupled device (CCD) (17) placed at a conjugate focal plane of the sample. The CCD camera (PIXIS: 100BR_eXcelon by Princeton Instruments, Inc.) is made of $1340 \times 100$ pixels 
(each $20 \mu \mathrm{m} \times 20 \mu \mathrm{m}$ ) with $100 \%$ fill factor and operated at $-75^{\circ} \mathrm{C}$ via thermoelectric cooling. With $80 \mathrm{x}$ magnification, each pixel corresponds a square of $250 \mu \mathrm{m} \times 250 \mu \mathrm{m}$ on the real sample.

The grating (1200 grooves $/ \mathrm{mm})$ in the spectrometer provides the needed diffraction power for resolving the emissions from different photon sources (e.g., different QDs), as shown by the CCD image in Figure 2.6(b). The data has been integrated for $10 \mathrm{~s}$ under solely above-band excitation ( $\mathrm{HeNe}$ ) of $5 \mathrm{nW}$. The fluorescence of a QD can be recognized by a bright center spot surrounded by a couple of diffraction rings caused by the small physical size of each QD. The light from many QDs is visible in this image. The emission from GaAs band edge (at around $880 \mathrm{~nm}$ ) has been filtered out through the grating diffraction and the glowing background in the image is probably caused by the continuous tail of the wetting layer defect states [130-132]. Since these data were recorded with the front slit (15) fully opened (to let through all the fluorescence), the wavelength reading of the bottom axis of Figure 2.6(b) is probably inaccurate. For accurately measuring of the emission wavelength, we can close down the slit (15) to an opening of less than $120 \mu \mathrm{m}$ and retake the image, as shown in Appendix A2.1.

If we excite the sample with the light resonant to one QD by using a cw laser (1), the image looks drastically different, as shown in Figure 2.6(c). Only one single QD can be observed, identified by the well-defined ring structure. The disappearance of other QDs and defect states implies that the energy structure of each QD is so specific that it is rare to find a second QD to have the same energy structure in the same area of the sample. The left inset of Figure 2.6(c) is a zoom-in image of the same data. The yellow line around the bright center spot circles an integration area to calculate the PL intensity. This area contains only 20\% of the QD fluorescence, whereas the center disk of an Airy function originated from pin-hole image holds $83 \%$ of intensity. This difference suggests that the object is not a point source, which would be for a bare quantum dot. This is consistent with the earlier discussion on wavelength-dependent output mode of the planar cavity in Section 2.1. Finally, an RPLE spectrum can be obtained by recording this area-integrated PL intensity at different excitation frequencies by scanning the excitation laser across the QD resonance energy, as shown in the right inset of Figure 2.6(c). The double peak structure in the spectrum implies a neutral QD with a fine structure splitting (FSS) of about 2.9 $\mathrm{GHz}$, which we label QD1 to refer to it later in this dissertation.

The spectrally-integrated PL can be output from the spectrometer through the other exit by flipping the last mirror in the spectrometer. The output light is firstly split in 50:50 ratio by coupling through a 50/50 singlemode fiber splitter (18), before being collected by a pair of single photon avalanche detectors (19) ( $\tau$-SPAD from PicoQuant). In practice, two avalanche photodiodes (APD) are used to overcome the dead-time limit ( $\sim 70 \mathrm{~ns}$ ) of each detector in order to perform a correlation experiment. These APDs are silicon-based and can detect 925 $\mathrm{nm}$ photons with an efficiency of $30 \%$ at a time resolution of 350 800 ps. The electronic pulses from these APDs are time-stamped by a fast timing circuit (PicoHarp 300 from PicoQuant) at a resolution of 4 pico-second. The accurate timing of each photon event allows us to extract a high-resolution correlation function $g^{(2)}(\tau)$ from the data with a home-written Matlab code (Appendix A2.13). Figure 2.6(d) gives an example of $g^{(2)}(\tau)$ extracted from the data collected on QD2, excited by HeNe laser at a power of $6.5 \mu \mathrm{W}$ ( $23 \%$ of saturation power). Note the correlation time (x-axis) is in logarithmic scale, covering 10 orders of magnitude. The anti-bunching dip at zero correlation time $(\tau=0)$ is $\sim 0.2$, below the classical limit of 1 , which confirms the QD's single- 
photon-emitter nature. The inset of Figure 2.6(d) shows the same data but processed with a linear scale code. The instrument response function (IRF), depicted in blue curve, reveals the response speed of the detectors. It is measured with pico-second pulses centered at $927.5 \mathrm{~nm}$, the same wavelength as the fluorescence of the QD2. The comparable width between IRF and $g^{(2)}(\tau)$ dip suggests a strong convolution effect occurs in the correlation measurement, and it has to be accounted when modeling the $g^{(2)}(\tau)$ at short time scale.

With pulsed excitation, time-resolved fluorescence experiment can be implemented to measure the lifetime $T_{1}$ of each QD. In this experiment, channel "start" on the timing module (20) is switched to a high-speed photodetector module (not shown) to track the arrival time of excitation pulses. Figure 2.6(e) shows an example measurement of lifetime of QD1. The data has been fitted with an exponential decay convolved with the measured IRF (blue curve), leading to a lifetime of $\mathrm{T}_{1}=565 \mathrm{ps}$ for QD1. 


\section{Chapter 3: Coherent Scattering from a Quantum Dot}

Summary. In typical epitaxially grown quantum dots, the anisotropic exchange interaction results in two bound exciton states split by several $\mu \mathrm{eV}$ with orthogonal transition dipole moments that emit linearly polarized fluorescence $[32,110,133,134]$. When the fine structure splitting is on the order of the transition linewidth, a cw excitation laser can interact with both exciton states simultaneously if it is polarized so as to have a non-zero projection onto both dipole moments. The QD-field interaction will cause both coherent scattering at the laser frequency [135-137] and incoherent spontaneous emission at the transition dipole frequencies. The fields coherently scattered from the two non-degenerate orthogonal dipoles will be at the same frequency but phaseshifted relative to each other. Interference between these fields results in a noticeable difference between the shapes of the excitation spectra for detection polarizations parallel and orthogonal to the excitation. This phenomenon is not present in pulsed excitation experiments, where the emission is generally unpolarized when both dipole moments are excited [138]. By measuring polarization-dependent excitation spectra for polarizations both aligned to the transition dipole moments and 45-degrees rotated relative to them, we can extract the real part of the coherence between the two fine structure states induced by the excitation [139]. 


\subsection{Semi-classical model: 3-level V-system}

Classically, the optical transition is modeled as a damped oscillator driven by an electric force associated with the excitation field. This model is successful in reproducing the Lorentzian line shape of the absorption spectrum of a 2-level system, but incapable of modeling excitation and emission spectrum due to its failure of incorporating spontaneous decay and coherent scattering. Specifically, correct modeling of power broadening exhibited in excitation spectra, and AC stark shift or Mollow triplet exhibited in emission spectra requires proper treatment of light-matter interaction, which can be achieved by using semi-classical formalism. Under this framework, two steps are mainly involved for calculating the laser-induced photoluminescence from a QD: 1) formulate the effect of the excitation field on the QD; 2) relate the perturbed QD state to the fluorescence field via the source-field relation. The first step is about excitation, where the neutral QD is modeled as a 3-level Vsystem quantum mechanically, while the excitation field is treated classically. The second step is about the radiation, where a full quantum mechanical description is used to include the spontaneous decay. Two excitation regimes will be discussed in this section: continuous-wave $(c w)$ excitation and pulsed excitation. We will show that the two excitation regimes lead to completely different polarization properties of the PL: no polarization for pulsed excitation versus strong polarization for cw excitation.

\subsubsection{Continuous-wave excitation regime}

As shown in Figure 3.1, the three energy levels of a neutral QD are denoted as $|0\rangle,|1\rangle$ and $|2\rangle$ with the associated energy given by $0, \hbar \omega_{1}$ and $\hbar \omega_{2}$, respectively. To the first-order approximation [140], a single-mode classical electromagnetic $(\mathrm{EM})$ wave at frequency $\omega$ interacts with the QD via its electric dipole moments:

$$
H_{E D}=e\left(\boldsymbol{d}_{1}+\boldsymbol{d}_{2}\right) \cdot \boldsymbol{E}_{0} \cos (\omega t)
$$

where $e$ is the electron charge, $\boldsymbol{E}_{0}$ is the amplitude of excitation field and $\boldsymbol{d}_{j}$ is the electric dipole moment of the QD associated with transition $\pi_{j}=|j\rangle\langle 0|$. In the basis of QD eigenstates $(|2\rangle,|1\rangle$ and $|0\rangle)$, the Hamiltonian of the whole system is (in Schrodinger picture)

$$
H=H_{A}+H_{E D}=\left(\begin{array}{ccc}
\hbar \omega_{2} & 0 & 0 \\
0 & \hbar \omega_{1} & 0 \\
0 & 0 & 0
\end{array}\right)+\left(\begin{array}{ccc}
0 & 0 & \hbar \Omega_{2} \\
0 & 0 & \hbar \Omega_{1} \\
\hbar \Omega_{2} & \hbar \Omega_{1} & 0
\end{array}\right) \cos (\omega t)
$$

where $\Omega_{j}=e \boldsymbol{d}_{j} \cdot \boldsymbol{E}_{0} / \hbar$ is the Rabi frequency associated with the transition $\pi_{j}$. The time-evolution of the system is governed by Liouville-von Neumann equation

$$
i \hbar \frac{d}{d t} \rho(t)=[H, \rho(t)]
$$

where $\rho(\mathrm{t})$ is a $3 \times 3$ density matrix describing the QD state in Schrodinger picture. By switching to a rotational frame (rotating at the laser frequency $\omega$ ) and using the rotating-wave approximation [140,141], Eqn. (3.3) gives a set of homogenous differential equations on each density-matrix element

$$
\begin{gathered}
2 i \dot{\tilde{\rho}}_{22}=\Omega_{2}\left(\tilde{\rho}_{02}-\tilde{\rho}_{20}\right) \\
2 i \dot{\tilde{\rho}}_{11}=\Omega_{1}\left(\tilde{\rho}_{01}-\tilde{\rho}_{10}\right) \\
2 i \dot{\tilde{\rho}}_{20}=-2 \delta_{2} \tilde{\rho}_{20}+\Omega_{2}\left(\tilde{\rho}_{00}-\tilde{\rho}_{22}\right)-\Omega_{1} \tilde{\rho}_{21}
\end{gathered}
$$


(a)

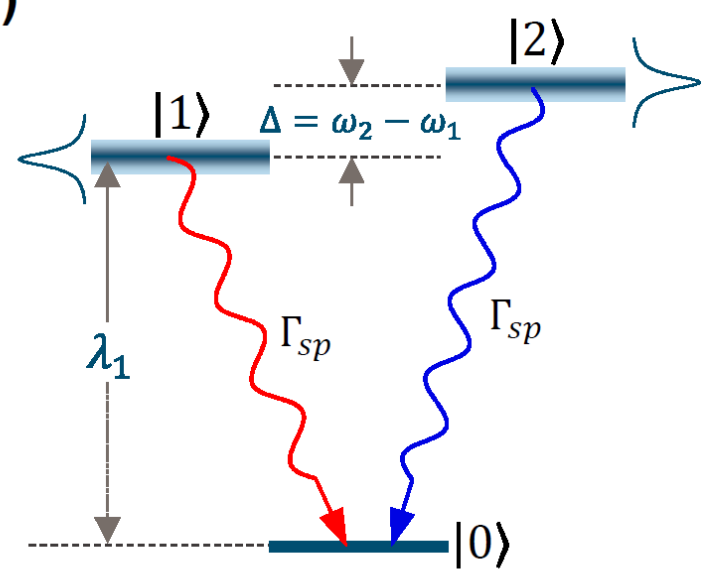

(b)

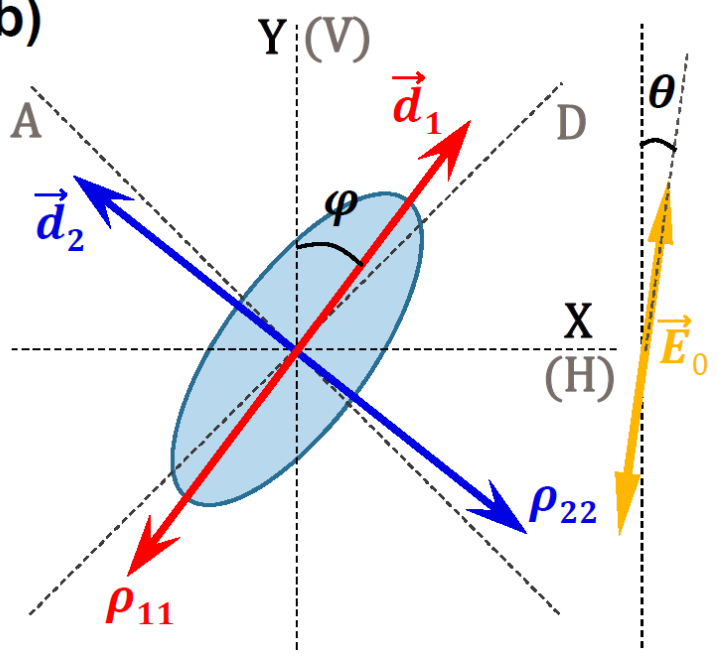

Figure 3.1 (a) Quantum dot energy diagram. For QD1, the transition wavelength for the low energy state $|1\rangle$ is $928.6088 \mathrm{~nm}$, the population spontaneous decay rate is determined to be $\Gamma_{\mathrm{sp}} / 2 \pi=(279.2 \pm 0.9) \mathrm{MHz}$ by time-resolved fluorescence measurements (see Section 3.2.1), and the fine structure splitting is $\Delta_{\mathrm{FSS}} / 2 \pi=$ $(2.869 \pm 0.001) \mathrm{GHz}$. (b) Polarization and electric dipole moment orientations. The lower energy $\boldsymbol{d}_{1}$ and higher energy $\boldsymbol{d}_{2}$ dipole moments are shown, as is the polarization of the excitation field $\mathbf{E}_{0}$. The shape of the QD is shown schematically with its asymmetry exaggerated.

$$
\begin{gathered}
2 i \dot{\tilde{\rho}}_{10}=-2 \delta_{1} \tilde{\rho}_{10}+\Omega_{1}\left(\tilde{\rho}_{00}-\tilde{\rho}_{11}\right)-\Omega_{2} \tilde{\rho}_{12} \\
2 i \tilde{\tilde{\rho}}_{21}=2 \Delta \tilde{\rho}_{21}+\Omega_{2} \tilde{\rho}_{01}-\Omega_{1} \tilde{\rho}_{20}
\end{gathered}
$$

where $\sim$ represents the rotating frame, $\delta_{1}=\omega-\omega_{1}, \delta_{2}=\omega-\omega_{2}$ are the laser detunings with respect to each transition, and $\Delta=\omega_{2}-\omega_{1}$ is the fine structure splitting between two neutral exciton states. The timeevolution for other four density-matrix elements $\left(\tilde{\rho}_{00}, \tilde{\rho}_{02}, \tilde{\rho}_{01}\right.$, and $\left.\tilde{\rho}_{12}\right)$ can be easily obtained by using the complex conjugate relation $\left(\tilde{\rho}_{j k}=\tilde{\rho}_{k j}^{\star}\right)$, and the normalization condition $\left(\tilde{\rho}_{00}+\tilde{\rho}_{11}+\tilde{\rho}_{22}=1\right)$. The dropped terms in the rotating-wave approximation are those that oscillate at double the laser frequency $\left(\propto e^{ \pm 2 i \omega t}\right)$, and which vanish quickly in the ensemble average.

Although Eqns. (3.4) incorporate both absorption and stimulated emission caused by the excitation field correctly, it still cannot describe the spontaneous emission caused by the vacuum EM field, because the Hamiltonian given by Eqn. (3.2) does not include any interaction between the QD and the vacuum mode. Without spontaneous decay, the excited population would be held in the excited state permanently (at least for a non-perturbed system), which is unphysical. Furthermore, the solid-state environment surrounding the QD fluctuates constantly and has a notable impact on the QD evidenced by the broadened line-width and intermittent fluorescence. These QDenvironment interactions can take place via various mechanisms, such as electron-phonon interactions, spin exchange interactions (between QD spin and nuclear-spins), and energy level shifts (caused by the charging dynamics of charge traps). It is almost impossible to suppress all these effects thoroughly. These observations basically lift the validity of assuming the QD as an isolated system, and require a modification of Eqns. (3.4) to include the environmental influence on the system. The formalism of a master equation in Lindblad form [142] meets these requests by adding extra dissipative terms to Eqn. (3.3) 


$$
i \hbar \frac{d}{d t} \rho=[H, \rho]+i \frac{\hbar}{2} \sum_{j=1,2}\left\{\Gamma_{s p} \mathcal{L}\left(\rho, \pi_{j}\right)+\Gamma^{\prime} \mathcal{L}\left(\rho, \pi_{j}^{\dagger} \pi_{j}\right)\right\}
$$

where the dissipative effects are included via Lindblad super-operators [143] for radiative decay and pure dephasing with rates $\Gamma_{\mathrm{sp}}$ and $\Gamma^{\prime}$, respectively: $\mathcal{L}(\rho, O)=O \rho^{\dagger} O-\left(\rho O^{\dagger} O+O^{\dagger} O \rho\right) / 2$. This leads to a modified version of Eqns. (3.4)

$$
\begin{gathered}
2 i \dot{\tilde{\rho}}_{22}=\Omega_{2}\left(\tilde{\rho}_{02}-\tilde{\rho}_{20}\right)-2 i \Gamma_{\mathrm{sp}} \tilde{\rho}_{22} \\
2 i \dot{\tilde{\rho}}_{11}=\Omega_{1}\left(\tilde{\rho}_{01}-\tilde{\rho}_{10}\right)-2 i \Gamma_{\mathrm{sp}} \tilde{\rho}_{11} \\
2 i \dot{\tilde{\rho}}_{20}=-2 \delta_{2} \tilde{\rho}_{20}+\Omega_{2}\left(\tilde{\rho}_{00}-\tilde{\rho}_{22}\right)-\Omega_{1} \tilde{\rho}_{21}-i\left(\Gamma_{\mathrm{sp}}+2 \Gamma^{\prime}\right) \tilde{\rho}_{20} \\
2 i \dot{\tilde{\rho}}_{10}=-2 \delta_{1} \tilde{\rho}_{10}+\Omega_{1}\left(\tilde{\rho}_{00}-\tilde{\rho}_{11}\right)-\Omega_{2} \tilde{\rho}_{12}-i\left(\Gamma_{\mathrm{sp}}+2 \Gamma^{\prime}\right) \tilde{\rho}_{10} \\
2 i \dot{\tilde{\rho}}_{21}=2 \Delta \tilde{\rho}_{21}+\Omega_{2} \tilde{\rho}_{01}-\Omega_{1} \tilde{\rho}_{20}-2 i\left(\Gamma_{\mathrm{sp}}+2 \Gamma^{\prime}\right) \tilde{\rho}_{21}
\end{gathered}
$$

where $\Gamma=\Gamma_{\mathrm{sp}} / 2+\Gamma^{\prime}$ is the total dephasing rate.

Equations (6) plus its complex conjugate counterparts gives a system of linear differential equations constrained by the normalization condition, $\tilde{\rho}_{00}+\tilde{\rho}_{11}+\tilde{\rho}_{22}=1$,

$$
\frac{d}{d t}\left(\begin{array}{l}
\tilde{\rho}_{22} \\
\tilde{\rho}_{11} \\
\tilde{\rho}_{00} \\
\tilde{\rho}_{21} \\
\tilde{\rho}_{12} \\
\tilde{\rho}_{20} \\
\tilde{\rho}_{02} \\
\tilde{\rho}_{10} \\
\tilde{\rho}_{01}
\end{array}\right)=\mathcal{M}\left(\begin{array}{c}
\tilde{\rho}_{22} \\
\tilde{\rho}_{11} \\
\tilde{\rho}_{00} \\
\tilde{\rho}_{21} \\
\tilde{\rho}_{12} \\
\tilde{\rho}_{20} \\
\tilde{\rho}_{02} \\
\tilde{\rho}_{10} \\
\tilde{\rho}_{01}
\end{array}\right)
$$

where the super-matrix $\mathcal{M}$ contains the coefficients of the system

$$
\mathcal{M}=\left(\begin{array}{ccccccccc}
-\Gamma_{\mathrm{sp}} & 0 & 0 & 0 & 0 & i \Omega_{2} / 2 & -i \Omega_{2} / 2 & 0 & 0 \\
0 & -\Gamma_{\mathrm{sp}} & 0 & 0 & 0 & 0 & 0 & i \Omega_{1} / 2 & -i \Omega_{1} / 2 \\
\Gamma_{\mathrm{sp}} & \Gamma_{\mathrm{sp}} & 0 & 0 & 0 & -i \Omega_{2} / 2 & i \Omega_{2} / 2 & -i \Omega_{1} / 2 & i \Omega_{1} / 2 \\
0 & 0 & 0 & -i \Delta-2 \Gamma & 0 & i \Omega_{1} / 2 & 0 & 0 & -i \Omega_{2} / 2 \\
0 & 0 & 0 & 0 & i \Delta-2 \Gamma & 0 & -i \Omega_{1} / 2 & i \Omega_{2} / 2 & 0 \\
i \Omega_{2} / 2 & 0 & -i \Omega_{2} / 2 & i \Omega_{1} / 2 & 0 & i \delta_{2}-\Gamma & 0 & 0 & 0 \\
-i \Omega_{2} / 2 & 0 & i \Omega_{2} / 2 & 0 & -i \Omega_{1} / 2 & 0 & -i \delta_{2}-\Gamma & 0 & 0 \\
0 & i \Omega_{1} / 2 & -i \Omega_{1} / 2 & 0 & i \Omega_{2} / 2 & 0 & 0 & i \delta_{1}-\Gamma & 0 \\
0 & -i \Omega_{1} / 2 & i \Omega_{1} / 2 & -i \Omega_{2} / 2 & 0 & 0 & 0 & 0 & -i \delta_{1}-\Gamma
\end{array}\right)
$$

The general time-dependent solutions to Eqn. (3.7) are quite complicated. However, for RPLE spectrum calculation, the simpler steady-state solution is enough, because the integration time of each data point in the RPLE spectrum $(\sim 3 \mathrm{~s})$ is much longer than the transient period of the system $(\sim 1 \mathrm{~ns})$. The steady-state solutions are labeled with an infinity superscript, like $\tilde{\rho}_{j k}^{\infty}$, in this dissertation. The analytical form of $\tilde{\rho}_{j k}^{\infty}$ is used in later calculations and data analysis, but it is too cumbersome and lengthy to list out here. One can use Mathematica to obtain these solutions directly. 
The next step is to relate these solutions to the emission intensity by using the source-field relation. The positive frequency part of the electric field operator for the scattered light is proportional to the lowering operators for the two excited states, $\pi_{1}$ and $\pi_{2}$, via (see Section 10.A in [141] or Section 7.8 in [140])

$$
\boldsymbol{E}^{(+)}(\boldsymbol{r}, t)=\sum_{j=1,2} \frac{\omega_{j}^{2}}{8 \pi^{2} \epsilon_{0} c^{2}|\boldsymbol{r}|} \boldsymbol{d}_{j} \pi_{j}\left(t-\frac{|\boldsymbol{r}|}{c}\right)
$$

where $\mathbf{r}$ is the vector from the scattering center (QD) to the observation point. The time-averaged polarizationindependent and dependent total intensities of the scattered light are

$$
\begin{gathered}
I=\frac{1}{2} \epsilon_{0} c\left\langle\boldsymbol{E}^{(-)}(\boldsymbol{r}, t) \cdot \boldsymbol{E}^{(+)}(\boldsymbol{r}, t)\right\rangle \\
I_{\varepsilon}=\frac{1}{2} \epsilon_{0} c\left\langle\left(\hat{\varepsilon} \cdot \boldsymbol{E}^{(-)}(\boldsymbol{r}, t)\right)\left(\hat{\varepsilon} \cdot \boldsymbol{E}^{(+)}(\boldsymbol{r}, t)\right)\right\rangle
\end{gathered}
$$

where $\mathbf{E}^{(-)}$is both the negative frequency part of the electric field operator and the Hermitian adjoint of $\mathbf{E}^{(+)}$, and $\hat{\varepsilon}$ is the unit vector corresponding to the detection polarization. The angled brackets represent both ensemble averaging and time averaging. In $I$ there will be terms proportional to $\pi_{j}^{\dagger} \pi_{j}$, but because of the orthogonality of the dipole moments there will be no cross terms. This is not the case for $I_{\varepsilon}$ because the dipole moments are turned into scalars via the dot product with $\hat{\varepsilon}$. Thus, the orthogonality of the dipole moments is no longer present to eliminate cross terms such as $\pi_{1}^{\dagger} \pi_{2}$. It is important to note that the orthogonality of the dipole moments is critical to the polarization dependence of the scattered light intensity. If the dipole moments were parallel to each other, as in an atomic system with spherical symmetry, then the cross terms would be present for all detection polarizations and thus the spectrum would have the same shape for all polarizations.

The ensemble-averaged values of the operator combinations in $I$ and $I_{\varepsilon}$ are equal to elements of the quantum mechanical density matrix of the system: $\left\langle\pi_{j}^{\dagger} \pi_{k}\right\rangle=\tilde{\rho}_{k j}(t)$. This allows us to express the detected emission intensity as a function of the density-matrix elements. The long integration time for each data point justifies the time-averaging of these solutions, leading to the steady-state solutions, $\tilde{\rho}_{j k}^{\infty}$. Depending on the detection polarization, the functional form for $I_{\varepsilon}$ will be different. Referring to Figure 3.1(b), for a given choice of $\hat{\varepsilon}$ the dot products in Eqn. (3.10) can be replaced by sinusoidal functions of $\varphi$.

For example, for X-polarized detection, Eqn. (3.10) becomes

$$
I_{\mathrm{X}}=\frac{1}{2} \epsilon_{0} c\left\langle\left(\hat{\mathrm{X}} \cdot \boldsymbol{E}^{(-)}(\boldsymbol{r}, t)\right)\left(\hat{\mathrm{X}} \cdot \boldsymbol{E}^{(+)}(\boldsymbol{r}, t)\right)\right\rangle
$$

Substitute Eqn. (3.8) into the above equation and notice $\left|\vec{d}_{1}\right| \approx\left|\vec{d}_{2}\right|=d$, and $\omega_{1} \approx \omega_{2} \approx\left(\omega_{1}+\omega_{2}\right) / 2$,

$$
\begin{gathered}
I_{\mathrm{X}}=\frac{1}{2} \epsilon_{0} c\left(\frac{\omega_{0}^{2} d}{8 \pi^{2} \epsilon_{0} c^{2} r}\right)^{2}\left\langle\left(\sin (\varphi) \pi_{1}^{\dagger}(t)-\cos (\varphi) \pi_{2}^{\dagger}(t)\right)\left(\sin (\varphi) \pi_{1}^{\dagger}(t)-\cos (\varphi) \pi_{2}^{\dagger}(t)\right)\right\rangle \\
=\frac{\omega_{0}^{4} d^{2}}{128 \pi^{4} \epsilon_{0} c^{3} r^{2}}\left\{\left\langle\pi_{1}^{\dagger}(t) \pi_{1}(t)\right\rangle \sin ^{2}(\varphi)+\left\langle\pi_{2}^{\dagger}(t) \pi_{2}(t)\right\rangle \cos ^{2}(\varphi)-\frac{1}{2} \sin (2 \varphi)\left[\left\langle\pi_{1}^{\dagger}(t) \pi_{2}(t)\right\rangle+\left\langle\pi_{2}^{\dagger}(t) \pi_{1}(t)\right\rangle\right]\right\}
\end{gathered}
$$

By applying $\left\langle\pi_{j}^{\dagger} \pi_{k}\right\rangle=\tilde{\rho}_{k j}(t)$ and noticing that $\tilde{\rho}_{j k}^{\infty}=\left(\tilde{\rho}_{k j}^{\infty}\right)^{\star}$, the above result becomes

$$
I_{\mathrm{X}}=\frac{\omega_{0}^{4} d^{2}}{128 \pi^{4} \epsilon_{0} c^{3} r^{2}}\left\{\tilde{\rho}_{11}^{\infty} \sin ^{2}(\varphi)+\tilde{\rho}_{22}^{\infty} \cos ^{2}(\varphi)-\frac{1}{2} \sin (2 \varphi)\left[\tilde{\rho}_{21}(t)+\tilde{\rho}_{12}(t)\right]\right\}
$$


The same derivation can be employed for Y-polarized and polarization-independent detection. The results can be transformed to the following functional forms by recognizing that $\tilde{\rho}_{j k}^{\infty}=\left(\tilde{\rho}_{k j}^{\infty}\right)^{\star}$ :

$$
\begin{gathered}
I_{\mathrm{X}}=I_{0}\left\{\tilde{\rho}_{11}^{\infty} \sin ^{2}(\varphi)+\tilde{\rho}_{22}^{\infty} \cos ^{2}(\varphi)-\operatorname{Re}\left[\tilde{\rho}_{12}^{\infty}\right] \sin (2 \varphi)\right\} \\
I_{\mathrm{Y}}=I_{0}\left\{\tilde{\rho}_{11}^{\infty} \cos ^{2}(\varphi)+\tilde{\rho}_{22}^{\infty} \sin ^{2}(\varphi)+\operatorname{Re}\left[\tilde{\rho}_{12}^{\infty}\right] \sin (2 \varphi)\right\} \\
I=I_{\mathrm{X}}+I_{\mathrm{Y}}=I_{0}\left\{\tilde{\rho}_{11}^{\infty}+\tilde{\rho}_{22}^{\infty}\right\}
\end{gathered}
$$

where $I_{0}$ is the intensity constant

$$
I_{0}=\frac{\omega_{0}^{4} d^{2}}{128 \pi^{4} \epsilon_{0} c^{3} r^{2}}
$$

Similarly, the RPLE intensity for other detection polarizations [diagonal (D), anti-diagonal (A), left- (L) and right-circular $(\mathrm{R})]$ are:

$$
\begin{gathered}
I_{\mathrm{D}}=\frac{1}{2} I_{0}\left\{\tilde{\rho}_{11}^{\infty}+\tilde{\rho}_{22}^{\infty}+\left(\tilde{\rho}_{11}^{\infty}-\tilde{\rho}_{22}^{\infty}\right) \sin (2 \varphi)-2 \operatorname{Re}\left[\tilde{\rho}_{12}^{\infty}\right] \cos (2 \varphi)\right\} \\
I_{\mathrm{A}}=\frac{1}{2} I_{0}\left\{\tilde{\rho}_{11}^{\infty}+\tilde{\rho}_{22}^{\infty}-\left(\tilde{\rho}_{11}^{\infty}-\tilde{\rho}_{22}^{\infty}\right) \sin (2 \varphi)+2 \operatorname{Re}\left[\tilde{\rho}_{12}^{\infty}\right] \cos (2 \varphi)\right\} \\
I_{\mathrm{L}}=\frac{1}{2} I_{0}\left\{\tilde{\rho}_{11}^{\infty}+\tilde{\rho}_{22}^{\infty}+2 \operatorname{Im}\left[\tilde{\rho}_{12}^{\infty}\right]\right\} \\
I_{\mathrm{R}}=\frac{1}{2} I_{0}\left\{\tilde{\rho}_{11}^{\infty}+\tilde{\rho}_{22}^{\infty}-2 \operatorname{Im}\left[\tilde{\rho}_{12}^{\infty}\right]\right\}
\end{gathered}
$$

where $\hat{\varepsilon}$ for left- and right-circular polarizations is defined as $(\hat{x}+i \hat{y}) / \sqrt{2}$ and $(\hat{x}-i \hat{y}) / \sqrt{2}$, respectively.

Generally, the scattered light from any single-mode excited system, such as a neutral QD excited by a narrowband cw laser, consists of two parts: coherent and incoherent scattering. The coherent part is made of photons identical to the excitation source, while the incoherent part is formed by spontaneous emission, which carries the optical information about the scattering center. The intensity of the coherent scattering can be calculated by [135],

$$
\begin{gathered}
I_{\text {tot }}^{\mathrm{coh}}=\frac{1}{2} \epsilon_{0} c\left\langle\boldsymbol{E}^{(-)}(\boldsymbol{r}, t)\right\rangle \cdot\left\langle\boldsymbol{E}^{(+)}(\boldsymbol{r}, t)\right\rangle \\
I_{\varepsilon}^{\mathrm{coh}}=\frac{1}{2} \epsilon_{0} c\left\langle\left(\hat{\varepsilon} \cdot \boldsymbol{E}^{(-)}(\boldsymbol{r}, t)\right)\right\rangle\left\langle\left(\hat{\varepsilon} \cdot \boldsymbol{E}^{(+)}(\boldsymbol{r}, t)\right)\right\rangle
\end{gathered}
$$

where $I_{\text {tot }}^{\text {coh }}$ is the total intensity of the coherent scattering with no polarization discrimination, and $I_{\varepsilon}^{\text {coh }}$ is the coherent scattering measured along polarization $\hat{\varepsilon}$.

Using the source-field relation (Eqn. (3.8)) with the ensemble average of the transition operator $\pi_{j}$ replaced by the density-matrix elements (similar to the method for deriving Eqns. (3.11) \& (3.12)), the coherent scattering proportion along $\mathrm{X}$ - or Y-polarization is determined to be

$$
\begin{aligned}
& I_{\mathrm{X}}^{\mathrm{coh}}=I_{0}\left|\tilde{\rho}_{01}^{\infty} \sin (\varphi)-\tilde{\rho}_{02}^{\infty} \cos (\varphi)\right|^{2} \\
& I_{\mathrm{Y}}^{\text {coh }}=I_{0}\left|\tilde{\rho}_{01}^{\infty} \cos (\varphi)+\tilde{\rho}_{02}^{\infty} \sin (\varphi)\right|^{2}
\end{aligned}
$$

The incoherent part is calculated by taking the difference between the total scattering and coherent scattering,

$$
\begin{aligned}
& I_{\mathrm{X}}^{\text {incoh }}=I_{0}\left\{\left(\tilde{\rho}_{11}^{\infty}-\left|\tilde{\rho}_{01}^{\infty}\right|^{2}\right) \sin ^{2}(\varphi)+\left(\tilde{\rho}_{22}^{\infty}-\left|\tilde{\rho}_{02}^{\infty}\right|^{2}\right) \cos ^{2}(\varphi)-\left(\operatorname{Re}\left[\tilde{\rho}_{12}^{\infty}\right]+\operatorname{Re}\left[\tilde{\rho}_{01}^{\infty}\left(\tilde{\rho}_{02}^{\infty}\right)^{\star}\right]\right) \sin (2 \varphi)\right\} \\
& I_{\mathrm{Y}}^{\text {incoh }}=I_{0}\left\{\left(\tilde{\rho}_{11}^{\infty}-\left|\tilde{\rho}_{01}^{\infty}\right|^{2}\right) \cos ^{2}(\varphi)+\left(\tilde{\rho}_{22}^{\infty}-\left|\tilde{\rho}_{02}^{\infty}\right|^{2}\right) \sin ^{2}(\varphi)+\left(\operatorname{Re}\left[\tilde{\rho}_{12}^{\infty}\right]+\operatorname{Re}\left[\tilde{\rho}_{01}^{\infty}\left(\tilde{\rho}_{02}^{\infty}\right)^{\star}\right]\right) \sin (2 \varphi)\right\}
\end{aligned}
$$




\subsubsection{Pulsed excitation regime}

In most pulsed excitation experiments, picosecond pulses are used to excite the QD either resonantly or quasiresonantly. Picosecond pulses are spectrally narrow to ensure low probability of exciting other QDs nearby and to guarantee that only the ground exciton state $E_{0}$ (see Figure 2.4(a)) can be excited. Temporally, each pulse is short, about three orders of magnitude shorter than the lifetime of an exciton state, to guarantee that the promoted electrons won't experience significant radiative decay before the excitation finishes. For a typical Ti:sapphire laser, the separation between two consecutive pulses is about $12 \mathrm{~ns}$, which is one order of magnitude longer than the lifetime such that the excited population in the QD would be completely depleted by radiative recombination before the next pulse arrives, i.e., , the QD is always in ground state $|0\rangle$ to interact with the pulses. Therefore, after pulse excitation, the QD is always in a same superposition state $|\psi\rangle$ that consists of both exciton states $(|1\rangle$ and $|2\rangle)$ and the ground state $|0\rangle$, written as

$$
|\psi\rangle=\beta e^{i \theta_{1}}|1\rangle+\beta e^{i \theta_{2}}|2\rangle+\sqrt{1-2 \beta^{2}}|0\rangle
$$

where $\beta$ is a real number determined by the pulse area, $\theta_{1}$ and $\theta_{2}$ are the relative phase of each excited state with respect to the ground state. Here, we assume that the pulse area is the same for both transitions of $|0\rangle$ to $|1\rangle$, or $|0\rangle$ to $|2\rangle$, as shown in Figure 3.1(a). State $|\psi\rangle$ is the initial state for QD to evolve after each pulse-excitation, and it defines the initial condition for density-matrix evolution:

$$
\tilde{\rho}_{0}=\left[\begin{array}{ccc}
\tilde{\rho}_{22}^{0} & \tilde{\rho}_{21}^{0} & \tilde{\rho}_{20}^{0} \\
\tilde{\rho}_{12}^{0} & \tilde{\rho}_{11}^{0} & \tilde{\rho}_{10}^{0} \\
\tilde{\rho}_{02}^{0} & \tilde{\rho}_{01}^{0} & \tilde{\rho}_{00}^{0}
\end{array}\right]=\left[\begin{array}{ccc}
\beta^{2} & \beta^{2} e^{i\left(\theta_{2}-\theta_{1}\right)} & \beta \sqrt{1-2 \beta^{2}} e^{i \theta_{2}} \\
\beta^{2} e^{i\left(\theta_{1}-\theta_{2}\right)} & \beta^{2} & \beta \sqrt{1-2 \beta^{2}} e^{i \theta_{1}} \\
\beta \sqrt{1-2 \beta^{2}} e^{-i \theta_{2}} & \beta \sqrt{1-2 \beta^{2}} e^{-i \theta_{1}} & 1-2 \beta^{2}
\end{array}\right]
$$

Note the initial condition is the same in the static frame and in the rotational frame.

The time-evolution of the system is still governed by Eqn. (3.7), except that all the Rabi frequencies in supermatrix $\mathcal{M}$ are equal to zero. This significantly simplifies Eqn. (3.7), and leads to the following solution

$$
\tilde{\rho}(t)=\left[\begin{array}{ccc}
\tilde{\rho}_{00}^{0}+\left(1-e^{-\Gamma_{\mathrm{sp}} t}\right)\left(\tilde{\rho}_{11}^{0}+\tilde{\rho}_{22}^{0}\right) & \tilde{\rho}_{01}^{0} e^{-\Gamma t} e^{-i \delta_{1} t} & \tilde{\rho}_{02}^{0} e^{-\Gamma t} e^{-i \delta_{2} t} \\
\tilde{\rho}_{01}^{0} e^{-\Gamma t} e^{i \delta_{1} t} & \tilde{\rho}_{11}^{0} e^{-\Gamma_{\mathrm{sp}} t} & \tilde{\rho}_{12}^{0} e^{-2 \Gamma t} e^{i \Delta t} \\
\tilde{\rho}_{20}^{0} e^{-\Gamma t} e^{i \delta_{2} t} & \tilde{\rho}_{21}^{0} e^{-2 \Gamma t} e^{-i \Delta t} & \tilde{\rho}_{22}^{0} e^{-\Gamma_{\mathrm{sp}} t}
\end{array}\right]
$$

where $\delta_{1}=\omega-\omega_{1}, \delta_{2}=\omega-\omega_{2}$ are the excitation detunings, $\Gamma_{\mathrm{sp}}$ is the radiative decay rate, $\Gamma$ is the total dephasing rate, and $\Delta=\omega_{2}-\omega_{1}$ is the fine structure splitting between the two exciton states.

By substituting the initial condition into Eqn. (3.25), we get the time-dependent solutions. The final detected PL intensity along polarization $\hat{\varepsilon}$ corresponds to the time integration of Eqn. (3.25) because the measurement is not time-resolved

$$
I_{\mathcal{E}}^{\text {Pulse }}=\frac{1}{2} \epsilon_{0} c \int_{0}^{\infty} d t\left\langle\left(\hat{\varepsilon} \cdot \boldsymbol{E}^{(-)}(\boldsymbol{r}, t)\right)\left(\hat{\varepsilon} \cdot \boldsymbol{E}^{(+)}(\boldsymbol{r}, t)\right)\right\rangle
$$

Therefore, for different detection polarizations, the measured PL intensity is 


$$
\begin{aligned}
& \bar{I}_{\mathrm{X}}^{\text {Pulse }}=\frac{\beta^{2} I_{0}}{\Gamma_{s p}}\left\{1-\left[\frac{\Gamma_{s p} \Gamma \cos \left(\theta_{1}-\theta_{2}\right)}{4 \Gamma^{2}+\Delta^{2}}-\frac{\Gamma_{\mathrm{sp}} \Delta \sin \left(\theta_{1}-\theta_{2}\right)}{4 \Gamma^{2}+\Delta^{2}}\right] \sin (2 \varphi)\right\} \\
& \bar{I}_{\mathrm{Y}}^{\text {Pulse }}=\frac{\beta^{2} I_{0}}{\Gamma_{s p}}\left\{1+\left[\frac{\Gamma_{s p} \Gamma \cos \left(\theta_{1}-\theta_{2}\right)}{4 \Gamma^{2}+\Delta^{2}}-\frac{\Gamma_{\mathrm{sp}} \Delta \sin \left(\theta_{1}-\theta_{2}\right)}{4 \Gamma^{2}+\Delta^{2}}\right] \sin (2 \varphi)\right\} \\
& \bar{I}_{\mathrm{D}}^{\text {Pulse }}=\frac{\beta^{2} I_{0}}{\Gamma_{s p}}\left\{1-\left[\frac{\Gamma_{s p} \Gamma \cos \left(\theta_{1}-\theta_{2}\right)}{4 \Gamma^{2}+\Delta^{2}}-\frac{\Gamma_{\mathrm{sp}} \Delta \sin \left(\theta_{1}-\theta_{2}\right)}{4 \Gamma^{2}+\Delta^{2}}\right] \cos (2 \varphi)\right\} \\
& \bar{I}_{\mathrm{A}}^{\text {Pulse }}=\frac{\beta^{2} I_{0}}{\Gamma_{s p}}\left\{1+\left[\frac{\Gamma_{s p} \Gamma \cos \left(\theta_{1}-\theta_{2}\right)}{4 \Gamma^{2}+\Delta^{2}}-\frac{\Gamma_{\mathrm{sp}} \Delta \sin \left(\theta_{1}-\theta_{2}\right)}{4 \Gamma^{2}+\Delta^{2}}\right] \cos (2 \varphi)\right\} \\
& \bar{I}_{\mathrm{L}}^{\text {Pulse }}=\frac{\beta^{2} I_{0}}{\Gamma_{s p}}\left\{1+\left[\frac{\Gamma_{s p} \Gamma \sin \left(\theta_{1}-\theta_{2}\right)}{4 \Gamma^{2}+\Delta^{2}}+\frac{\Gamma_{\mathrm{sp}} \Delta \cos \left(\theta_{1}-\theta_{2}\right)}{4 \Gamma^{2}+\Delta^{2}}\right]\right\} \\
& \bar{I}_{\mathrm{R}}^{\text {Pulse }}=\frac{\beta^{2} I_{0}}{\Gamma_{s p}}\left\{1-\left[\frac{\Gamma_{s p} \Gamma \sin \left(\theta_{1}-\theta_{2}\right)}{4 \Gamma^{2}+\Delta^{2}}+\frac{\Gamma_{\mathrm{sp}} \Delta \cos \left(\theta_{1}-\theta_{2}\right)}{4 \Gamma^{2}+\Delta^{2}}\right]\right\}
\end{aligned}
$$

where $I_{0}$ is the intensity constant given by Eqn. (3.14). Considering $\Delta \approx 5 \Gamma_{\mathrm{sp}}$ and $\Gamma>\Gamma_{\mathrm{sp}}$, the second term is much smaller than 1 and can be neglected. This leads to an equal emission intensity for all polarizations, i.e., the PL is not polarized. 


\subsection{Experiments}

We use the optical setup in Figure 2.6 to record the RPLE spectra of QD1 at different detection polarizations controlled by the LCVRs. Ideally, no optics between the QD and the LCVRs should alter the polarization state of the light so that the collected data reflects the actual polarization state emitted by the QD. However, three mirrors oriented $45^{\circ}$ with respect to the incident light introduce polarization-dependent phase-retardance and power absorption that change the polarization state of the light after reflection. In Stokes parameter context, this effect is described by a $4 \times 4$ Mueller matrix that can be characterized and measured experimentally [144]. We characterized the Mueller matrix of the collection path using a laser of the same wavelength as the PL and with multiple polarization states (see Appendix A3.1). The inverse of the resulting Mueller matrix is applied to the measured polarization-dependent data to recover the original light polarization as it was emitted by the QD. The accuracy of the final spectra thus not only depends on the uncertainty of the collected spectra but also relies on the uncertainty of the measured Mueller matrix. It is difficult to suppress the latter because of the voltagedependent shift of the fast axis of each LCVR, as discussed in Section 2.3. Therefore, it is preferable to analyze the data directly without invoking the Mueller matrix to convert the data. For our setup, the light with horizontal $(\mathrm{X})$ or vertical $(\mathrm{Y})$ polarizations does not experience any polarization rotation but only power absorptions at different levels, because these two polarizations are the two principal axes of the PL path. Thus, we can analyze these data directly by using the model described in Section 3.1, with the absorption difference treated as a free parameter in fitting. Nevertheless, for Stokes parameter analysis, the Mueller matrix still has to be applied.

As in previous resonant excitation experiments [61,145], a small amount of above band-gap excitation is needed to allow resonance fluorescence from the neutral exciton state by neutralizing the intrinsic charges captured by the QD. The fluorescence of QD1 studied in this work is 23 times brighter when introducing $2.19 \mathrm{nW}$ of HeNe laser light $(632 \mathrm{~nm})$ onto the sample compared to the case of no above band-gap illumination. This amount of HeNe power is applied during the collection of all the spectra data presented in this section, and it corresponds to a fraction of $1.17 \times 10^{-5}$ times the above band-gap saturation power $\mathrm{P}_{1}=187.5 \mu \mathrm{W}$. I will discuss the effect of the above-band excitation on the QD in detail in Chapter 4.

\subsubsection{Carrier recombination in quantum dot: lifetime measurement}

The lifetime $\mathrm{T}_{1}$ of the QD1 is measured with time-resolved fluorescence (setup given in Section 2.3), wherein the QD1 is excited by 2.36 ps long resonant pulses centered at $927.604 \mathrm{~nm}$ with a time-averaged power of $13 \mu \mathrm{W}$. The PL is recorded with a single photon avalanche detector. This experiment is carried out at three different above band-gap excitation powers: $1.17 \times 10^{-5} \mathrm{P}_{1}, 3.33 \times 10^{-6} \mathrm{P}_{1}$, and $1.01 \times 10^{-6} \mathrm{P}_{1}$, as shown in Figure 3.2. The data are then fitted with an exponential decay convolved with the measured instrument response function [IRF is given in Figure 2.6(e)]. The lifetime $\mathrm{T}_{1}$ is determined to be the statistical average of these three fitting results: $T_{1}=(570 \pm 9)$ ps, since $T_{1}$ shows no dependence on the above-band excitation power [145]. 


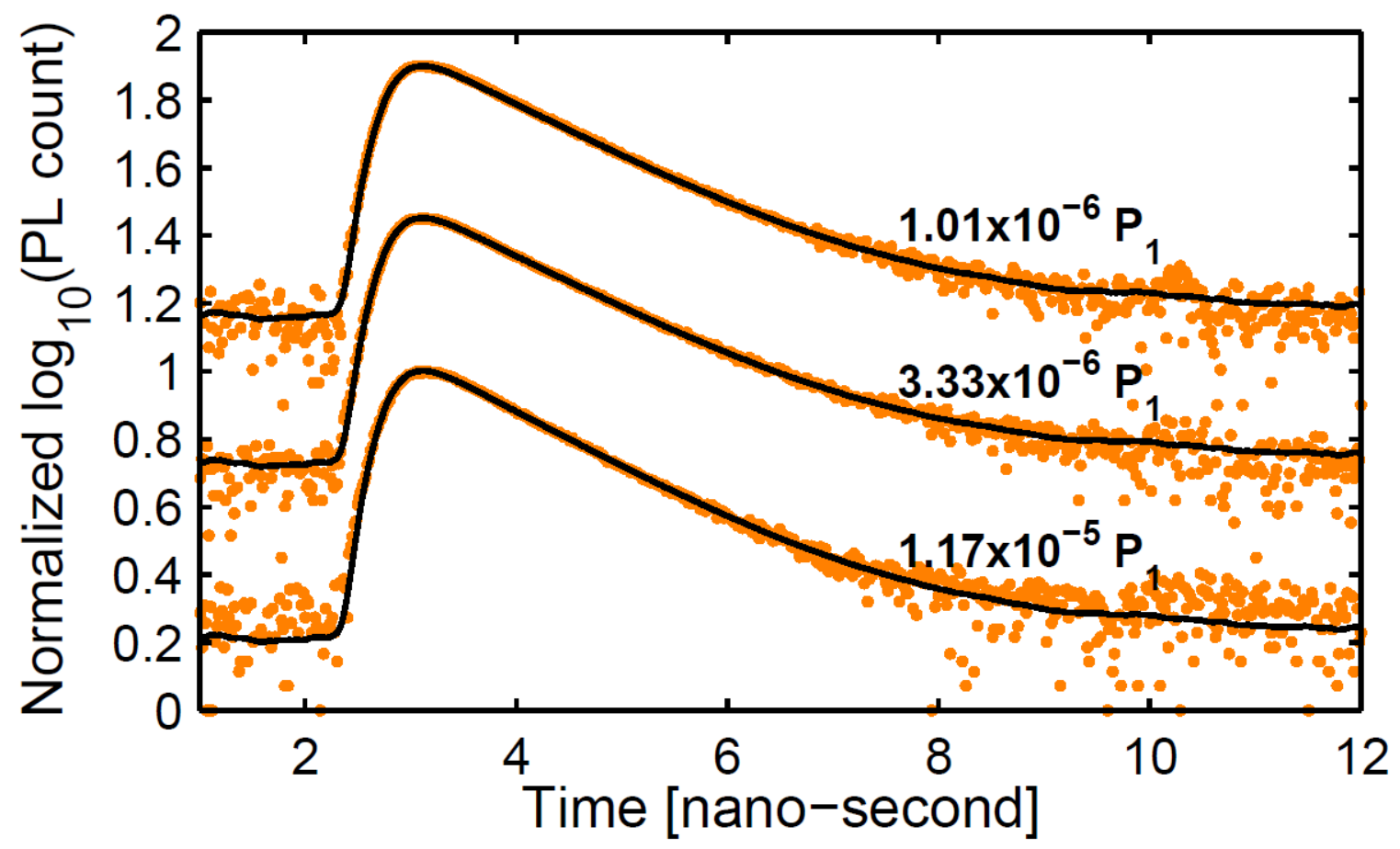

Figure 3.2 Lifetime Measurement. The orange dots are the raw data. The black curves are the fittings with an exponential decay convolved with the measured instrument response function (IRF). The power indicated is that of the weak above-band excitation required to allow resonance fluorescence, where $\mathrm{P}_{1}=187.5 \mu \mathrm{W}$; the resonant excitation power is $13 \mu \mathrm{W}$. The lifetime does not vary with above-band power, and is determined to be the average of the three fitted values: $T_{1}=(570 \pm 9)$ ps.

\subsubsection{Resonant fluorescence excitation spectroscopy}

Figure 3.3(a-b) show two normalized RPLE spectra under the same excitation conditions but with different detection polarizations: horizontal $(\mathrm{X})$ or vertical $(\mathrm{Y})$. Neither spectrum can be reconstructed by incoherently adding two Lorentzian lines centered at the two peaks as would be the case if the fluorescence consisted solely of spontaneous emission. This implies that to account for the observed unconventional line shapes, we must also include a polarization-dependent interference occurring between photons coherently scattered by the two fine structure states. Clear evidence of the presence of such interference can be seen at zero detuning, where a $90^{\circ}$ polarization switching with respect to the excitation field is present, i.e., the scattered light becomes highly Xpolarized even though the excitation is Y-polarized.

As suggested by Eqns. (3.11) \& (3.12), the RPLE intensities are not just proportional to the excited state populations $\rho_{11}^{\infty}$ and $\rho_{22}^{\infty}$, but are modified by the real part of the coherence between the two excited states, $\operatorname{Re}\left[\rho_{12}^{\infty}\right]$. In contrast, the total PL intensity in Eqn. (3.13) is still proportional to the total population in both excited states. The difference in the sign of the third terms in $I_{X}$ and $I_{Y}$ explains the difference between the Xpolarized and Y-polarized RPLE spectra. We rewrite Eqns. (3.11) \& (3.12) with fitting parameters 

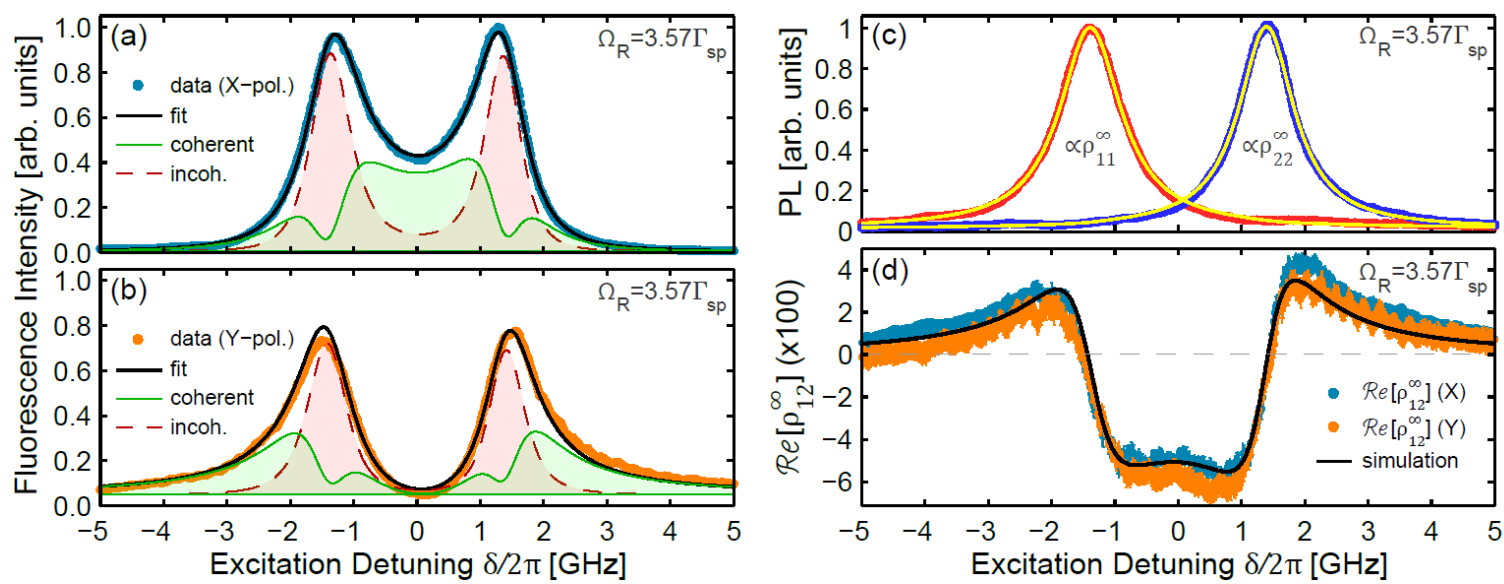

Figure 3.3 Example RPLE spectra recorded with $\Omega_{\mathrm{R}}=3.57 \Gamma_{\mathrm{sp}}$ and detection polarization chosen to be (a) $\mathrm{X}$ (blue points), (b) Y (orange points), or (c) to eliminate either the low-energy or high-energy peak. The black solid lines in (a) and (b) are fittings following Eqns. (3.28) \& (3.29). The green (solid) and red (dashed) lines underneath the spectra are the calculated portions of coherent and incoherent scattering, respectively. The two yellow curves in (c) are the fittings obtained by using the calculated exciton populations $\rho_{11}^{\infty}$ and $\rho_{22}^{\infty}$ from the 3-level model. (d) The coherence $\operatorname{Re}\left[\rho_{12}^{\infty}\right]$ extracted from the curves in (a) and (b); the colors indicate the polarization whence the coherence was extracted. The black solid curve in (d) is not a fit but the calculation of $\operatorname{Re}\left[\rho_{12}^{\infty}\right]$ using the same parameters found in the previous fittings in (a) and (b).

$$
\begin{aligned}
& I_{X}=A_{X}\left\{\tilde{\rho}_{11}^{\infty} \sin ^{2}(\varphi)+\tilde{\rho}_{22}^{\infty} \cos ^{2}(\varphi)-\operatorname{Re}\left[\tilde{\rho}_{12}^{\infty}\right] \sin (2 \varphi)\right\}+B_{X} \\
& I_{Y}=A_{Y}\left\{\tilde{\rho}_{11}^{\infty} \cos ^{2}(\varphi)+\tilde{\rho}_{22}^{\infty} \sin ^{2}(\varphi)+\operatorname{Re}\left[\tilde{\rho}_{12}^{\infty}\right] \sin (2 \varphi)\right\}+B_{Y}
\end{aligned}
$$

where $A_{X}$ and $A_{Y}$ are the spectrum amplitudes, and $B_{X}$ and $B_{Y}$ are the backgrounds. By simultaneously fitting these equations to multiple sets of RPLE spectra measured at different excitation powers, we determine the orientation of the dipole moment $\boldsymbol{d}_{1}$ to be $\varphi=44.74^{\circ} \pm 0.04^{\circ}$ with respect to the Y-axis, and the direction of the resonant excitation field $\boldsymbol{E}_{0}$ to be $\theta=3.37^{\circ} \pm 0.07^{\circ}$ with respect to the Y-axis. Thus the electric dipole moments of the QD1, $\boldsymbol{d}_{1}$ and $\boldsymbol{d}_{2}$, are almost aligned to the diagonal (D) and the anti-diagonal (A) directions, respectively, which is consistent with our Stokes parameter measurement and analysis of the PL (see Section 3.2.3). $\boldsymbol{E}_{0}$ is nominally aligned to the Y-axis because the excitation laser is propagating in the X-direction along the waveguide mode. But $\boldsymbol{E}_{0}$ may deviate from that alignment due to unintentional non-normal incidence of the laser on the air-GaAs interface, which would cause refraction of the beam away from the X-direction. In the fitting, the two spectrum amplitudes, $A_{X}$ and $A_{Y}$, are related to each other by a global parameter $\alpha$ via $A_{Y}=\alpha A_{X}$, which originates from the polarization-dependent absorption of the PL path. Direct measurement of the transmission coefficients along the PL path determines this ratio to be $0.8067 \pm 0.0598$, which is in close agreement with the fitting result of $0.799 \pm 0.003$.

To get an insight into the underlying physics, an analysis of the proportions of coherent and incoherent scattering in these spectra is helpful. By evaluating Eqns. (3.21) \& (3.22) with the above fitting parameters, these proportions are plotted in Figure 3.3(a-b), denoted by the green (solid) and red (dashed) lines for coherent and incoherent scattering, respectively. As expected, the incoherent scattering looks much like the incoherent sum of two Lorentzians; the small overlap of the two peaks leads to a negligible contribution at zero detuning. In contrast, 
because the coherent scattering is always at the laser wavelength, a pronounced interference effect is expected between contributions from the two dipoles. The relative phase shift of the coherently scattered photons is determined by the detuning of the laser with respect to each transition energy. This is similar to a driven harmonic oscillator: red-detuned driving results in a negative (lagging) phase while blue-detuned driving leads to a positive (leading) phase. Thus at zero detuning, the fields coherently scattered from the two dipoles have a relative phase shift even though the field polarizations are still aligned to each dipole. The detection polarization determines whether these phase-shifted fields combine constructively (for horizontal (X) polarization) or destructively (for vertical (Y) polarization). This explains the observed enhancement or diminution of the PL signal around zero detuning in Figure 3.3(a-b), respectively.

Because of this interference, the coherent scattering performance of a 3-level system can probably be better than a single 2-level structure, according to the calculation of coherent scattering intensity and percentage from these two systems as shown in Figure 3.4. The blue curve represents the 3-level system excited at zero-detuning, with excitation field at $45^{\circ}$ with respect to its dipole moments and detection polarization orthogonal to the excitation polarization. This is similar to the case of QD1 [Figure 3.1(a)] with $\theta=0^{\circ}, \varphi=45^{\circ}$, and X-polarized detection.
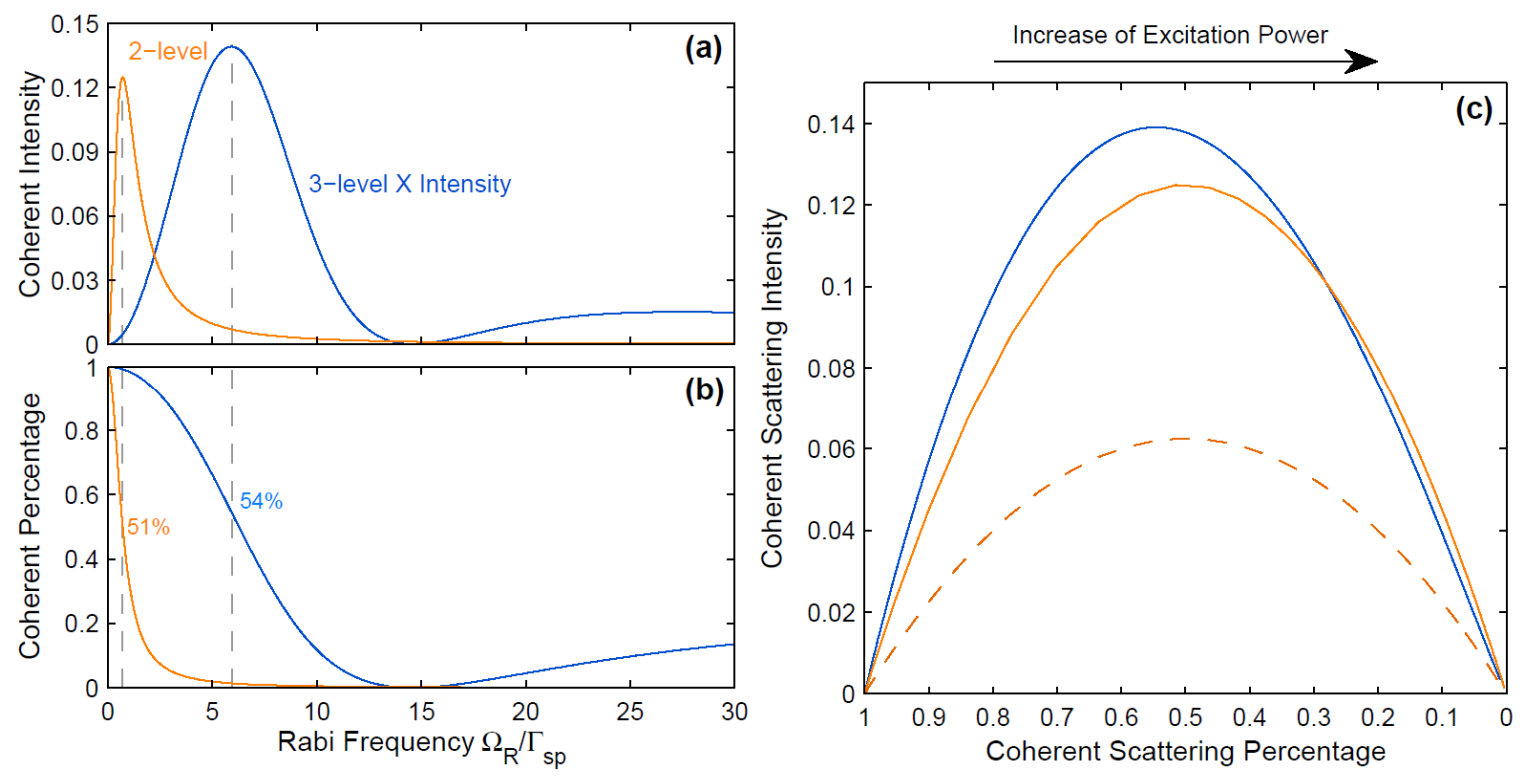

Figure 3.4 Comparison of the calculated coherent scattering from a 3-level system and a 2-level system. Parameters for 3-level system calculation: QD1 energy structure, 45-deg orientation of the excitation field with respect to each, and X-polarization detection. Parameters for 2-level system calculation: both excitation field and detection polarization are aligned to the dipole moment. (a) Coherent scattering intensity. (b) Coherent scattering percentage. The vertical dashed grey lines depicts the maximum coherent scattering intensity of each system. (c) Plot of coherent intensity in (a) versus the coherent percentage in (b), within the Rabi frequency of $14.6 \Gamma_{\mathrm{sp}}$. The solid orange and blue curves denote the same 2-level and 3-lelve systems as shown in (a) and (b). The dashed orange curve represents the 2-level system excited with a polarization at $45^{\circ}$ with respect to its dipole and detected at the orthogonal polarization with respect to the excitation polarization. This configuration excitation-detection scheme effectively blocks the laser scattering, while sacrificing half of coherent scattering intensity. 
The solid orange curve denotes the 2-level system under resonant excitation, with both excitation and detection polarizations aligned to its dipole moment. For a better comparison, we extract the calculated coherent scattering intensities of both systems from (a) and the coherent scattering percentages from (b) in the excitation power of $0<\Omega_{\mathrm{R}}<14.6 \Gamma_{\mathrm{sp}}$, and plot the exctracted coherent scattering intensity versus percentage in Figure 3.4(c). Within this power range, the coherent scattering percentages of both systems experience a monotonic decrease as the increase of the excitation power. For a given coherent scattering percentage, the 3-level system can always output more coherent light that 2-level system. For maximum coherent scattering intensity $(0.14$ for 3 -level, 0.125 for 2 -level), the purity of coherent scattering of 3 -level system is $3 \%$ higher than 2 -level system ( $54 \%$ vs. $51 \%$ ). If we match the coherent scattering intensity of the 3-level system to the maximum coherent scattering intensity of the 2-level system, the purity of 3-level scattering can reach $70 \%$. It is worth mentioning that the calculation of 2-level system does not consider any practical method to suppress the strong laser scattering, for example, using cross excitation detection polarizations to block the laser scattering. This method will decrease the coherent scattering intensity of 2-level by a factor of two, as shown by the dashed orange curve in Figure 3.4(c). If we match the coherent scattering intensity of the 3-level system to this lower maximum value, the coherent scattering percentage can be as high as $89 \%$. This is a great improvement of coherent scattering performance and worth an experimental confirmation for future work. In experiment, the coherent portion of the fluorescence could be separated out of scattering and measured with heterodyne setup $[74,137]$. This would also provide us an opportunity to verify the theoretical predictions shown in Figure 3.3(a-b) and in Figure 3.4.

\subsubsection{Steady-state coherence measurement: $\operatorname{Re}\left[\rho_{12}^{\infty}\right]$}

Figure 3.3(c) shows the RPLE spectra measured with the LCVRs tuned to block the PL emitted from either the high-energy or low-energy state of the fine structure doublet. Since that approach measures the emission from only one energy level at a time, there is no interference effect in these spectra. Each spectrum in Figure 3.3(c) can be directly fitted with the corresponding excited population (denoted as solid yellow lines) following $\mathrm{I}_{1}=$ $\mathrm{a}_{1} \rho_{11}^{\infty}+b_{1}$ and $\mathrm{I}_{2}=a_{2} \rho_{22}^{\infty}+b_{2}$ for states $|1\rangle$ and $|2\rangle$, respectively, where $a_{\mathrm{j}}$ and $b_{\mathrm{j}}$ are fitting parameters. Meanwhile, the sum of the two spectra in Figure 3.3(c) should be identical to the sum of the two spectra in Figure 3.3(a-b), in the sense that both sums are proportional to the total excited population in the QD. However, this observation is true only if the polarization-dependent absorption of the PL path is corrected. We perform this comparison using the following formula:

$$
\frac{I_{X}^{E X P}-B_{X}}{\beta_{X}}+\frac{I_{Y}^{E X P}-B_{Y}}{\beta_{Y}}=\frac{I_{1}^{E X P}-B_{1}}{\beta_{1}}+\frac{I_{2}^{E X P}-B_{2}}{\beta_{2}}
$$

where $I_{X}^{E X P}$ and $I_{Y}^{E X P}$ are the experimental RPLE spectra in Figure 3.3(a-b), $I_{1}^{E X P}$ and $I_{2}^{E X P}$ are the two measured spectra in Figure 3.3(c), $\beta_{X}=0.944, \beta_{Y}=0.762$ are the experimentally determined transmission coefficients of the PL path for X-and Y-polarization, $B_{X}$ and $B_{Y}$ are the spectrum backgrounds found in the earlier global fitting by using Eqn. (3.28) \& (3.29), $\beta_{1}$ and $\beta_{2}$ are the two global fitting parameters for all the spectra taken at different excitation powers, while $B_{1}$ and $B_{2}$ are the local parameters for a single excitation power. This global fitting allows us to determine $\beta_{1}=0.839 \pm 0.002, \beta_{2}=0.849 \pm 0.002$, and to extract the real part of the coherence between the fine structure states, $\operatorname{Re}\left[\tilde{\rho}_{12}^{\infty}\right]$, from Eqn. (3.28) \& (3.29): 


$$
\begin{aligned}
& \operatorname{Re}\left[\tilde{\rho}_{12}^{\infty}\right]_{X}=-\frac{1}{2 A_{X}}\left\{I_{X}^{E X P}-B_{X}-\frac{\beta_{X}}{\beta_{1}}\left(I_{1}^{E X P}-B_{1}\right) \tan (\varphi)-\frac{\beta_{X}}{\beta_{2}}\left(I_{2}^{E X P}-B_{2}\right) \cot (\varphi)\right\} \\
& \operatorname{Re}\left[\tilde{\rho}_{12}^{\infty}\right]_{Y}=+\frac{1}{2 A_{Y}}\left\{I_{Y}^{E X P}-B_{Y}-\frac{\beta_{Y}}{\beta_{1}}\left(I_{1}^{E X P}-B_{1}\right) \cot (\varphi)-\frac{\beta_{Y}}{\beta_{2}}\left(I_{2}^{E X P}-B_{2}\right) \tan (\varphi)\right\}
\end{aligned}
$$

where $A_{X}$ and $A_{Y}$ are the same amplitude found in Eqn. (3.28) \& (3.29). These expressions give the extracted $\operatorname{Re}\left[\tilde{\rho}_{12}^{\infty}\right]$ shown in Figure 3.3(d). Using parameters from the fits in Figure 3.3(a-b), the 3-level V-system simulation reproduces the shape of the coherence successfully (black curve in Figure 3.3(d)).

Figure 3.5 shows the extracted real part of the coherence for multiple excitation powers. As the excitation power increases, the dispersive line shapes centered at each fine structure resonance increase in magnitude and experience power broadening as is expected for coherent excitation [146]. The green lines are the coherence calculated by modeling the neutral QD as a double 2-level system (See Appendix A3.2), which apparently underestimates the coherence between the two excited states. Comparatively, the 3-level simulations (black lines)

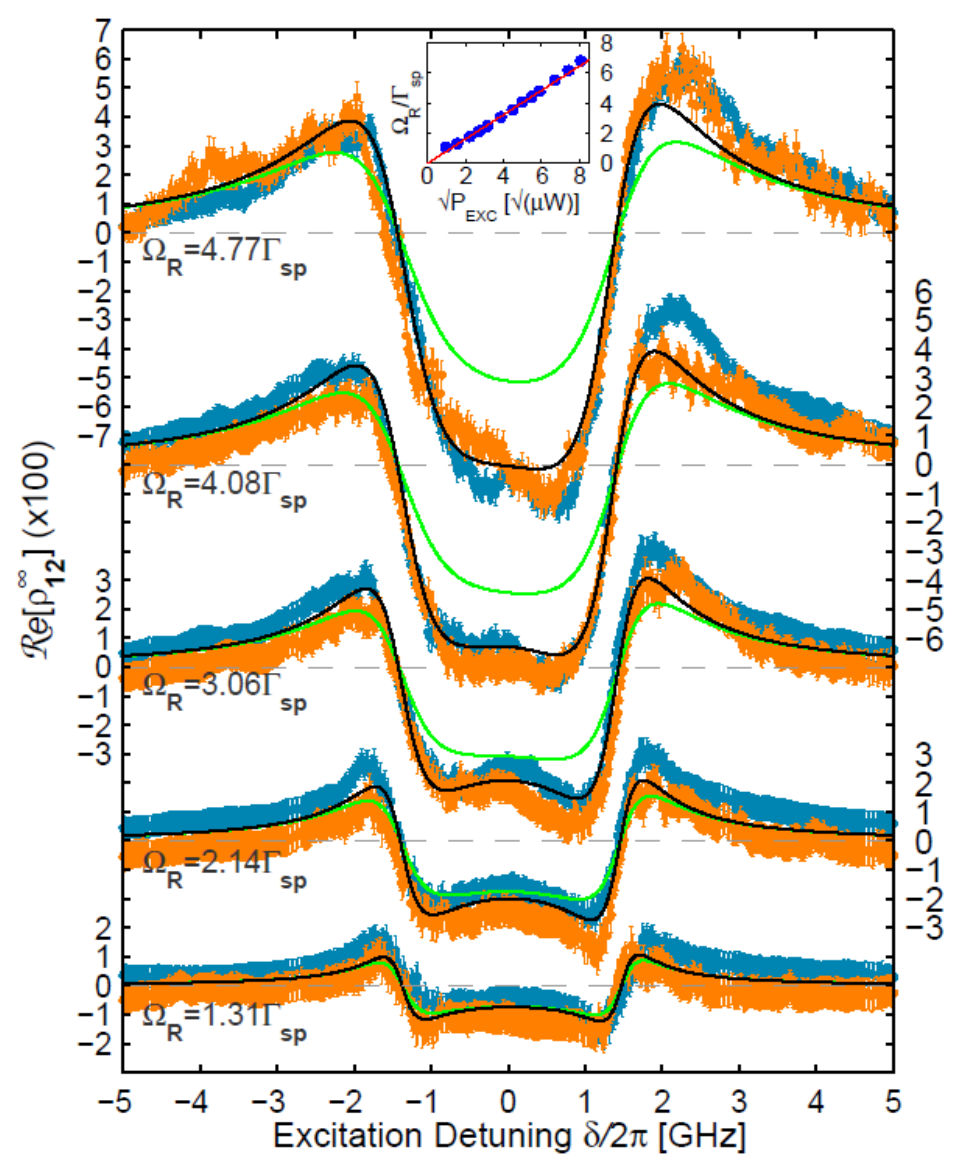

Figure 3.5 $\operatorname{Re}\left[\rho_{12}^{\infty}\right]$ extracted at different excitation powers. The curves are vertically offset for clarity. The color scheme used in each pair is the same as Figure 3.3(d), i.e., orange is extracted from the Y-polarized RPLE and blue from X-polarized. The black solid curves are the 3-level calculation of $\operatorname{Re}\left[\rho_{12}^{\infty}\right]$ at different $\operatorname{Rabi}$ frequencies $\Omega_{\mathrm{R}}$ determined by fittings to the raw RPLE data similar to Figure 3.3(a-b). The other model parameters are the same throughout all the calculations. The green curves are the similar calculations but based on the double 2-level model given in Appendix A3.2. Inset: Rabi frequency $\Omega_{\mathrm{R}}$ vs. the square root of the excitation power. The red straight line is a linear fit with a slope of $(0.806 \pm 0.011) \Gamma_{\mathrm{sp}} / \sqrt{\mu \mathrm{W}}$. 
match the data well and even reproduce the slight asymmetry about zero detuning. We note that to obtain an observable asymmetry requires two conditions: (1) the dipole moments of the QD must not be oriented 45 degrees with respect to the excitation field, and (2) the excitation power must be high. The single condition of tilted QD dipole moments is not enough to achieve this asymmetry according to the simulations of a heavily tilted QD with $\varphi=10^{\circ}$, as shown in Figure 3.6(a). With this tilting angle, the Rabi frequencies of the two transitions are significantly different, but it is clear that the coherence $\operatorname{Re}\left[\rho_{12}^{\infty}\right]$ is nearly symmetric at low excitation power $\Omega_{R}=0.579 \Gamma_{\mathrm{sp}}$. As the power increases $\left(\Omega_{R}>\Gamma_{\mathrm{sp}}\right), \operatorname{Re}\left[\rho_{12}^{\infty}\right]$ becomes more and more asymmetric, suggesting that this is a non-linear effect happening at high excitation power. In the inset of Figure 3.5, the Rabi frequencies extracted from the fittings follow a linear relationship with respect to the square root of power, as expected.

There are several differences between the $\mathrm{cw}$ excitation used in this study and experiments that use pulsed excitation to create and measure coherence (e.g., ref. [138]). Under pulsed excitation, a coherent superposition of two excited states is created by the excitation pulse, which then evolves freely over time and experiences quantum beats at a frequency determined by the fine structure splitting $\Delta_{\mathrm{FSS}}$. Since $\Delta_{\mathrm{FSS}} \gg \Gamma_{\mathrm{sp}}$, it most often leads to a vanishingly small polarization in the time-integrated fluorescence as indicated by Eqn. (3.27). In addition, the fluorescence is entirely spontaneous emission, which is incoherent; there is no coherent scattering. In contrast, under $\mathrm{cw}$ excitation coherent scattering occurs and a strong polarization results. Moreover, the density matrix under $\mathrm{cw}$ excitation is in a steady-state that depends on the excitation power and detuning, rather than a time-varying state.
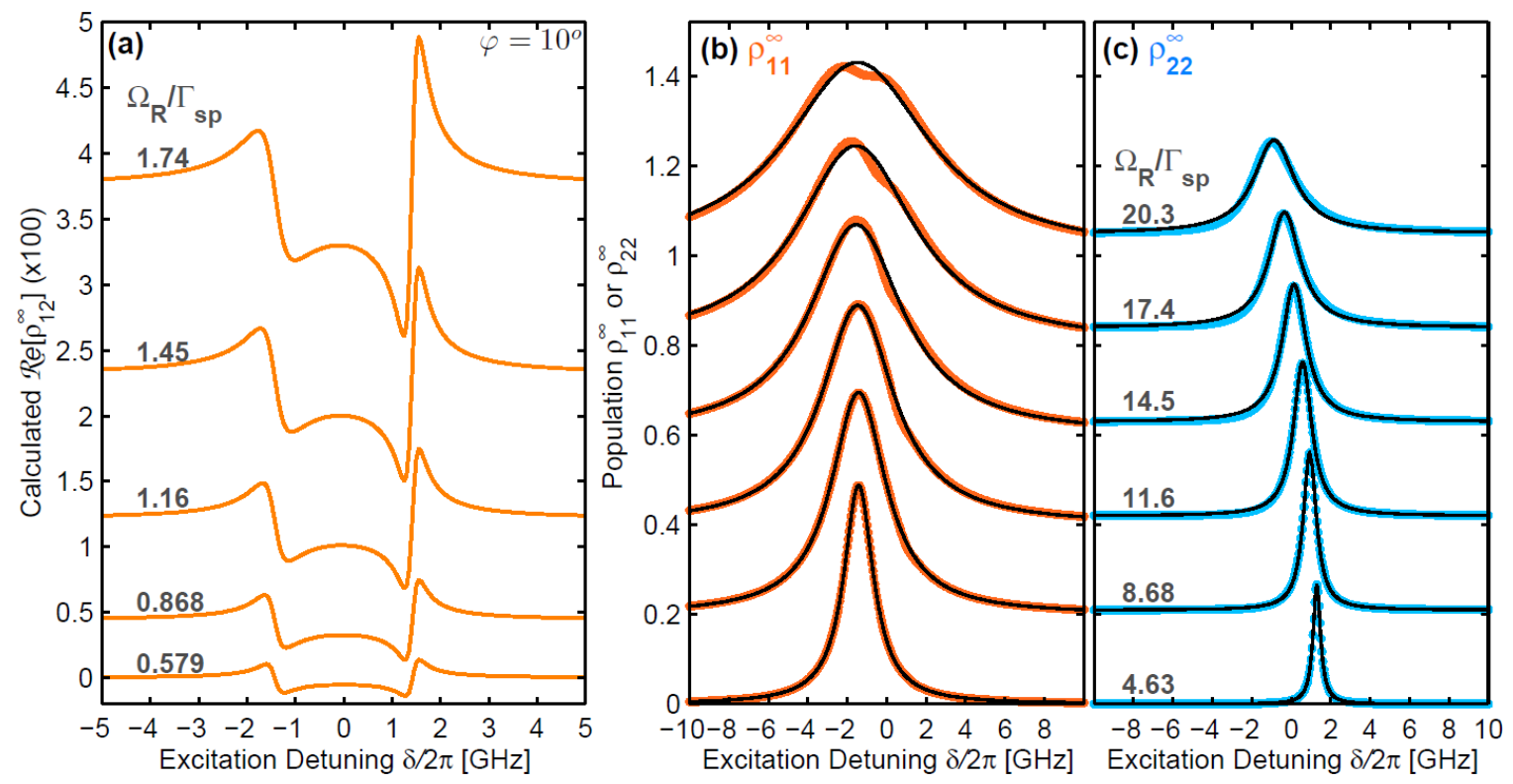

Figure 3.6 Calculated coherence $\operatorname{Re}\left[\rho_{12}^{\infty}\right]$ by setting the QD's electric dipole moment $\boldsymbol{d}_{1}$ to be $10^{\circ}$ off from the excitation field $\boldsymbol{E}_{0}\left(\theta=0^{\circ}\right)$. (b) Calculated population $\rho_{11}^{\infty}$ in the low energy excited state $|1\rangle$ (thick orange curves) along with the Lorentzian fit (black curves). (c) Calculated population $\rho_{22}^{\infty}$ in the high energy excited state $|2\rangle$ (thick blue curves) along with the Lorentzian fit (black curves). 


\subsubsection{Steady-state coherence measurement: $\operatorname{Im}\left[\rho_{12}^{\infty}\right]$}

According to Eqns. (3.17) and (3.18), it is possible to measure the imaginary part of the coherence by collecting the RPLE spectra at left- and right-circular polarizations, based on $\mathrm{I}_{L}-\mathrm{I}_{\mathrm{R}} \propto \operatorname{Im}\left[\rho_{12}^{\infty}\right]$. This requires to use the Mueller matrix $\left(M_{c o l}\right)$ of the collection path to recover the original polarization state $S_{i}$ from the measured final polarization state $S_{f}$, due to the presence of strong polarization rotation on circularly polarized light,

$$
S_{i}=M_{c o l}^{-1} S_{f}
$$

where $S_{i}$ and $S_{f}$ are the Stokes vector defined as

$$
S=\left(\begin{array}{c}
I \\
Q \\
U \\
V
\end{array}\right)=\left(\begin{array}{c}
\left\langle E_{X}^{2}\right\rangle+\left\langle E_{Y}^{2}\right\rangle \\
\left\langle E_{X}^{2}\right\rangle-\left\langle E_{Y}^{2}\right\rangle \\
\left\langle E_{D}^{2}\right\rangle-\left\langle E_{A}^{2}\right\rangle \\
\left\langle E_{L}^{2}\right\rangle-\left\langle E_{R}^{2}\right\rangle
\end{array}\right)
$$

where $E_{X}, E_{Y}, E_{D}, E_{A}, E_{L}$ and $E_{R}$ are the electric field intensity along the subscript directions. Thus, we collect other four RPLE spectra at D, A, L, and R detection polarizations. Along with the two spectra in Figure 3.3(a-b), the Stokes vector $S_{f}$ can be constructed based on Eqn. (3.34), and $S_{i}$ can be derived by using Eqn.

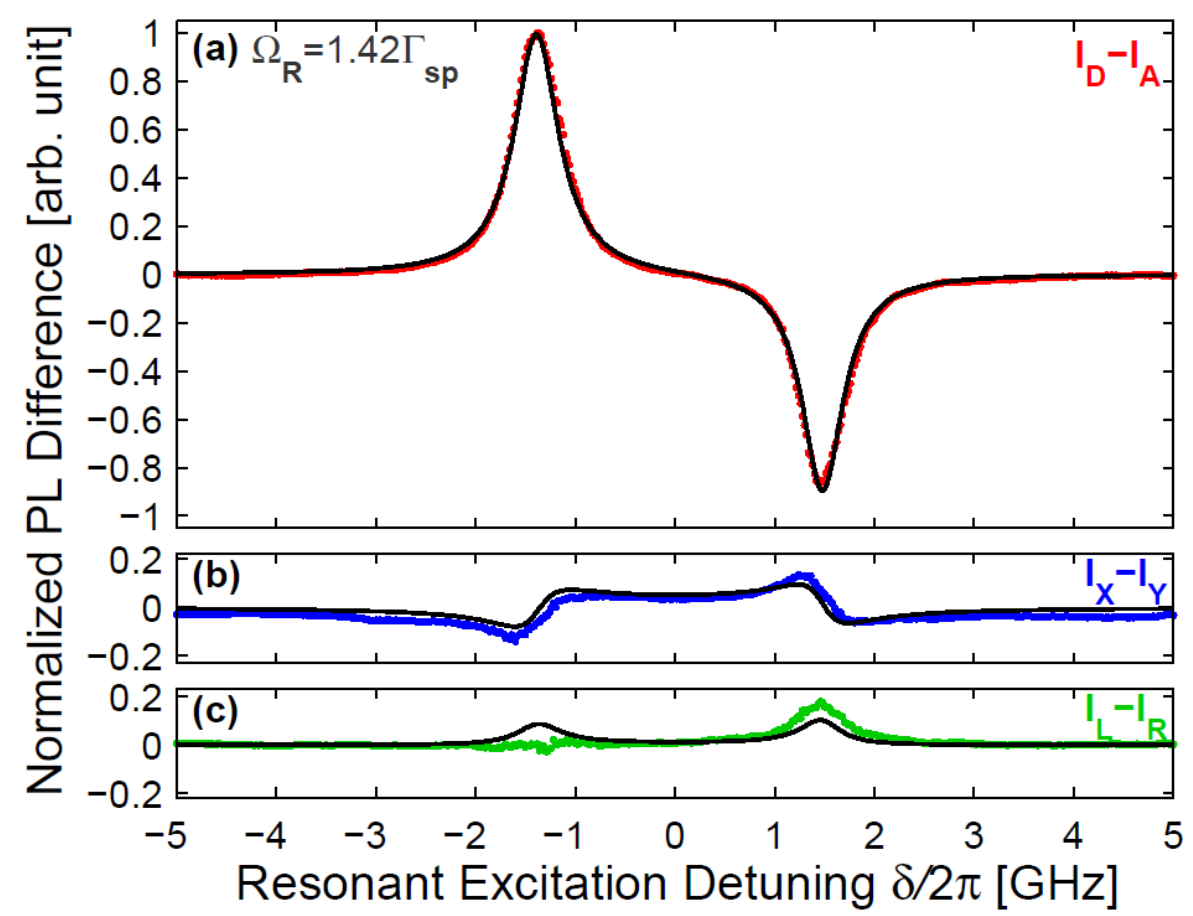

Figure 3.7 Differences between RPLE spectra measured at an excitation power of $\Omega_{R}=1.42 \Gamma_{\mathrm{sp}}$. (a) Intensity difference between $D$ - and A-polarized spectra $\left(I_{D}-I_{A}\right)$. (b) Intensity difference between X- and Y-polarized spectra $\left(I_{X}-I_{Y}\right)$. (c) Intensity difference between left- and right-circular polarized spectra $\left(I_{L}-I_{R}\right)$. The black curves are simulations using parameters extracted from a fit similar to the one done in Figure 3.3(a-b). All experimental data are normalized to the maximum intensity of the D-polarized spectrum. Before doing the subtraction between two spectra, the rotation of the polarization caused by the optics in the collection path has been corrected by inverting the Mueller matrix of the collection path upon the measured Stokes vector. 
(3.33) with $M_{\text {col }}$ given in Appendix A3.1. Figure 3.7(a-c) show the corrected spectra along with the 3-level model simulations using parameters from the fittings in Figure 3.3(a-b). The red curve in Figure 3.7(a) is the difference between the $\mathrm{D}$ and A polarization components of the fluorescence. The fact that it dominates over the curves in Figure 3.7(b-c), which are the X-Y and L-R differences, respectively, confirms that the QD asymmetry axes are indeed closely aligned to the diagonal and anti-diagonal directions. The small but non-zero X-Y component in Figure 3.7(b) is due to imperfect alignment of electric dipole moments to the diagonal and antidiagonal directions. The non-zero L-R component in Figure 3.7(c) implies elliptically polarized emission at the resonances, probably due to the heavy-hole and light-hole admixture [147-152]. An evident deviation of the corrected data from the simulation is present in Figure 3.7(c), which basically prevents the accurate extraction of $\operatorname{Im}\left[\rho_{12}^{\infty}\right]$ from the data. The main source of error here is the uncertainty of the Mueller matrix.

Nevertheless, we can calculate $\rho_{12}^{\infty}$ with the 3-level model and obtain some insights to its excitation power and detuning dependence by plotting its the magnitude and phase separately, as shown in Figure 3.8. This simulation covers the whole experimental power range from $0.1 \Gamma_{\mathrm{sp}}$ to $6.78 \Gamma_{\mathrm{sp}}$ (indicated by the horizontal dashed lines), and extends up to $\Omega_{R}=20 \Gamma_{\mathrm{sp}}$, which is difficult for an experiment to handle, because of the significant wavelength- dependent laser scattering at high excitation power. When the excitation is in resonance with one

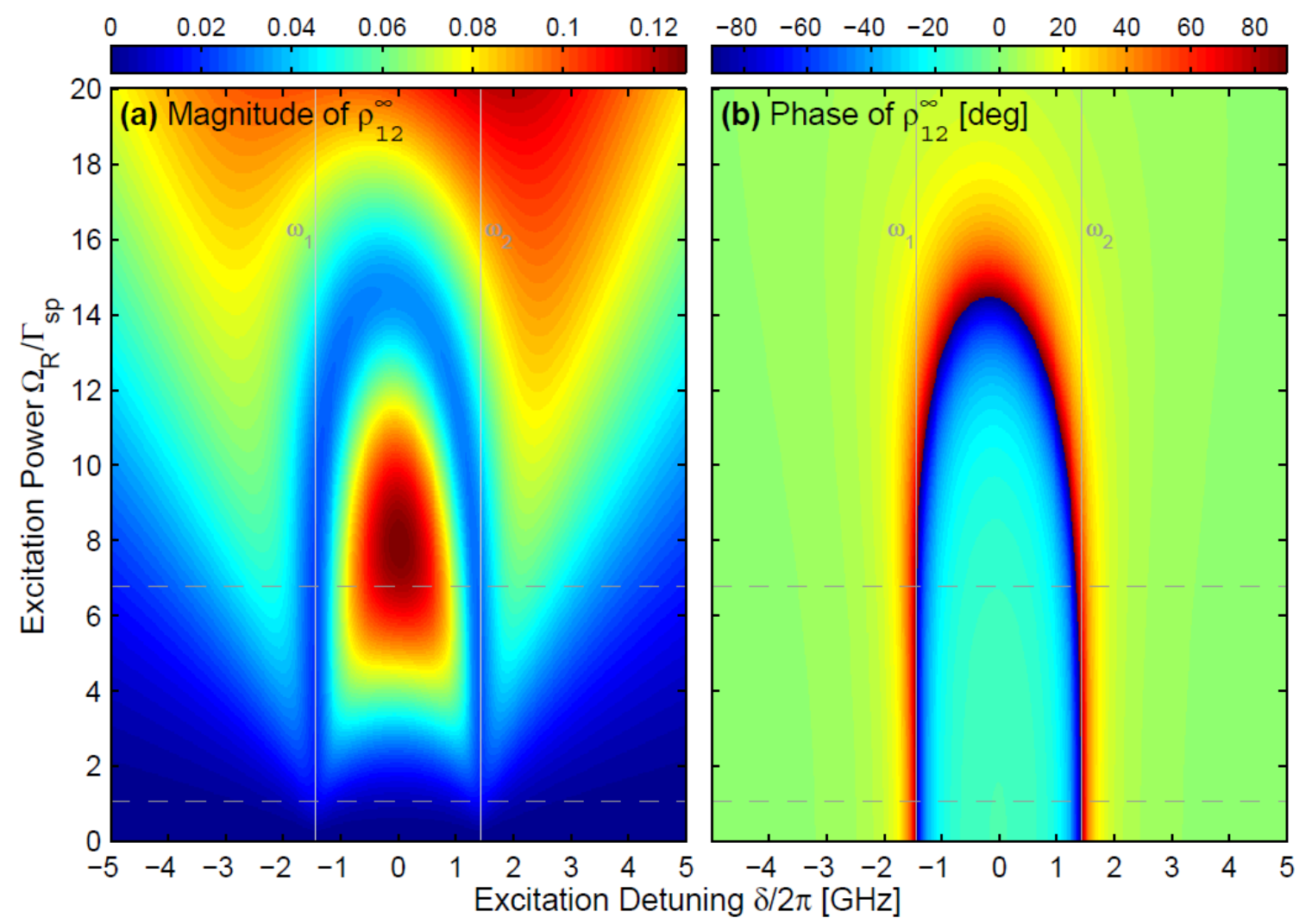

Figure 3.8 Simulated coherence $\rho_{12}^{\infty}$ : (a) magnitude, and (b) phase. The calculation is based on the 3-level model with QD1 parameters. Zero detuning (x-axis) is set to be the mean value of the two exciton transitions. The two horizontal dashed grey lines in each plot indicate the power range used for experiment, i.e., from $1.05 \Gamma_{\mathrm{sp}}$ to $6.78 \Gamma_{\mathrm{sp}}$. The two vertical lines give the position of the intrinsic transition energies $\left(\omega_{1} / 2 \pi\right.$ and $\left.\omega_{2} / 2 \pi\right)$ of the QD. 
transition $\left(\omega_{1}\right.$ or $\left.\omega_{2}\right)$, the coherence drops and a sharp phase switching happens (sign flip), as shown in Figure 3.8(b). At zero-detuning, the coherence is enhanced when excitation power increases from 0 to $8 \Gamma_{\text {sp }}$, implying that the off-resonant excitation photons might play an important role in creation and build-up of the coherence between the two exciton states. From 8 to $14 \Gamma_{\mathrm{sp}}$, the coherence decreases, probably due to the powerbroadening effect, where the photons can trigger more spontaneous decay. Above $14 \Gamma_{\mathrm{sp}}$, the increase of the excitation power improves the coherence possibly via the enhancement of the stimulated emissions.

\subsubsection{AC Stark shifts}

Each spectrum in Figure 3.3(c) can also be fitted with a Lorentzian function, as shown in Figure 3.9. The goodness of these fittings indicates that a 2-level quantum emitter model is a good approximation for the population of a single exciton level in the neutral QD. In fact, at low excitation power $\left(\Omega_{\mathrm{R}}<\Gamma_{\mathrm{sp}}\right)$, the double 2level system (See Appendix A3.2) can simulate the scattering by the neutral QD quite precisely, as evidenced by the close matching of the calculation (green curves) to the extracted coherence at lowest excitation power in
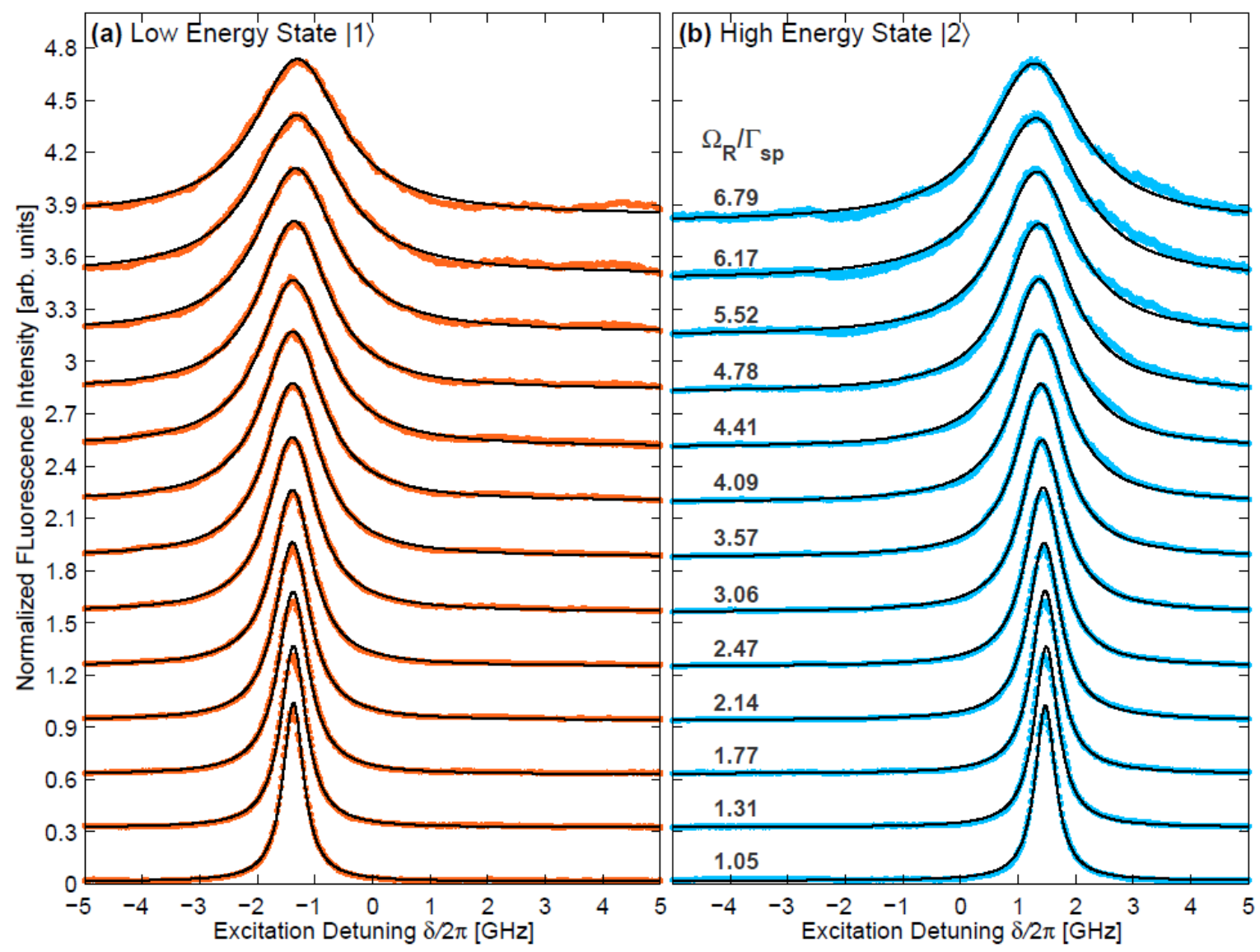

Figure 3.9 Lorentzian fits to the measured populations (a) $\rho_{11}^{\infty}$ and (b) $\rho_{22}^{\infty}$. The colored dots are the raw data and the black curves are fits with Lorentzian functions. All data have been normalized to their own maximum values. The curves are offset purposely for clarity. The excitation powers are labeled in the overall Rabi frequency $\Omega_{\mathrm{R}}$ in units of the population decay rate $\Gamma_{\mathrm{sp}}$ of QD1. 


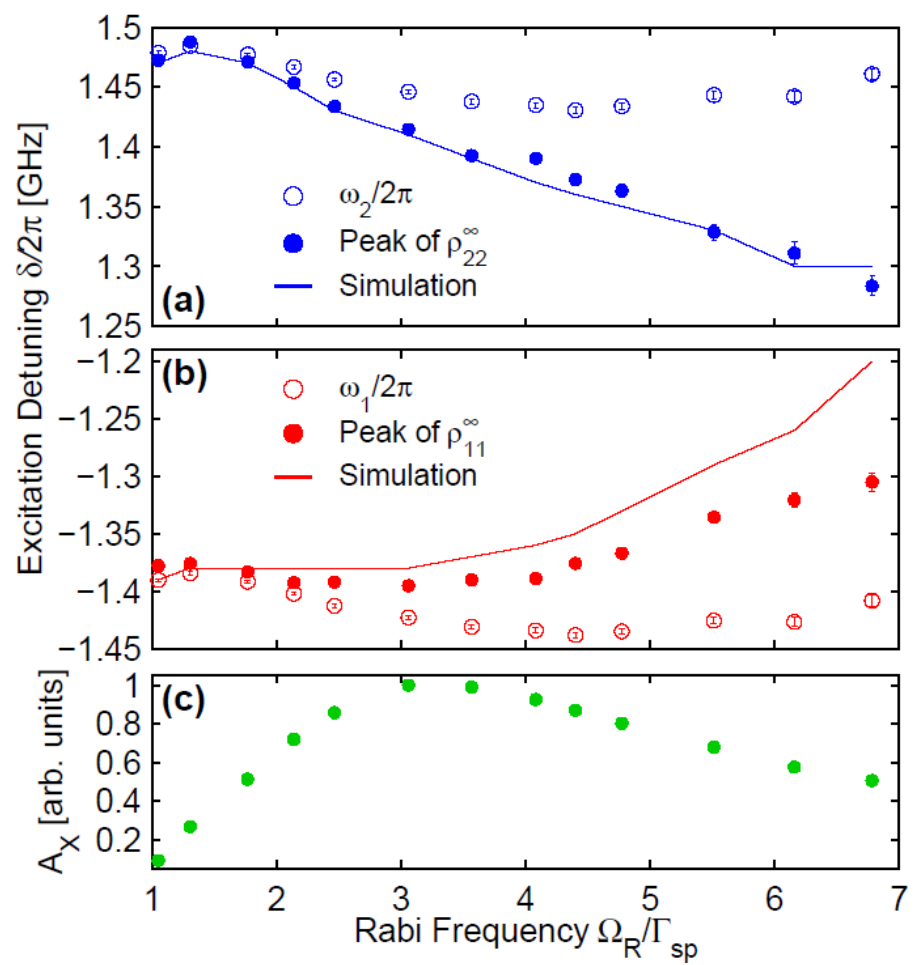

Figure 3.10 (a) The evolution of the high-energy state's intrinsic resonance frequency $\omega_{2} / 2 \pi$ (blue open circles) and the evolution of the peak positions of the excited population $\rho_{22}^{\infty}$ obtained by either a Lorentzian fitting (blue dots) or the V-system simulation (blue line). (b) The evolution of the low-energy state's intrinsic resonance frequency $\omega_{1} / 2 \pi$ (red open circles) and the evolution of the peak positions of the excited population $\rho_{11}^{\infty}$ obtained by either a Lorentzian fitting (red dots) or the $\mathrm{V}$-system simulation (red line). (c) Normalization factor $\mathrm{A}_{\mathrm{X}}$ of $\mathrm{X}$-polarized spectra at different excitation powers.

Figure 3.5. However, it is worth pointing out that the Lorentzian fitting is not always perfect, especially for high excitation power, as shown in Figure 3.6(b-c). The simulated populations in the excited states start to deviate from Lorentzian shape when the excitation power goes over than $\Omega_{\mathrm{R}} \cos (\varphi) \geq 9.8 \Gamma_{\mathrm{sp}}$.

The solid dots in Figure 3.10(a-b) are the peak positions of the Lorentzian functions in Figure 3.9. We find that the two resonance peaks move towards each other as the power increases; this is due to the AC Stark effect. For example, when the laser is near resonance with the low-energy state $|1\rangle$, the excitation field is red-detuned with respect to the high-energy state $|2\rangle$. This detuning pushes state $|0\rangle$ and state $|2\rangle$ away from each other via the AC Stark effect [153-155]. The red-shift of the ground state in turn effectively increases the transition energy of state $|1\rangle$. Since the AC Stark effect gets stronger at higher excitation power, the low-energy state $|1\rangle$ moves continuously towards the higher energy side of the spectrum. Similarly, the high-energy state $|2\rangle$ experiences a red-shift in its transition energy as the power increases. We calculate the expected resonance positions based on the fitting parameters found in Figure 3.3(a) and depicted them as solid lines in Figure 3.10(a-b). They are in good agreement with the data, especially for the high-energy state. 
Similar phenomenon is also present in the simulation in Figure 3.6(b-c), where the excited population of the high-energy state $\rho_{22}^{\infty}$ shows an evident red-shift of its peak position due to the AC Stark effect, while the peak position of the excited population of the low-energy state $\rho_{11}^{\infty}$ hardly moves. Due to the heavily tilting of the QD, the Rabi frequency $\Omega_{1}$ for level $|1\rangle$ is 5.6 times of the Rabi frequency $\Omega_{2}$ for level $|2\rangle$ under the same excitation power, i.e., $\Omega_{1}=\Omega_{2} \cot (\varphi)=5.6 \Omega_{2}$. This leads to a stronger AC Stark effect on level $|1\rangle$ compared to that on level $|2\rangle$, resulting in a clear red-shift of $\rho_{22}^{\infty}$ and a nearly stationary $\rho_{11}^{\infty}$.

The open circles in Figure 3.10(a-b) represent the fitting parameters $\omega_{2} / 2 \pi$ and $\omega_{1} / 2 \pi$, called the intrinsic transition frequencies for states $|2\rangle$ and $|1\rangle$. These parameters are determined by the intrinsic properties of the QD, which should not change if the QD's local environment is not disturbed. Therefore, the variation of $\omega_{2} / 2 \pi$ and $\omega_{1} / 2 \pi$ shown in Figure 3.10(a-b) reflects the fluctuations of the local environment caused by the resonant excitation laser. More evidence for the influence of the resonant laser on the local environment is given by the fitting parameter $A_{X}$, the normalization constant of the X-polarized spectra, as shown in Figure 3.10(c). Since the V-system model completely incorporates the population variation in the excited states $|1\rangle$ and $|2\rangle$ for different excitation powers and detunings, one might naively expect $A_{X}$ to be a constant that only depends on the exposure time and collection efficiency rather than on the resonant excitation power. However, its evident dependence upon excitation power implies that the resonant laser must also modify the fraction of time that the QD spends in the neutral state. This behavior is consistent with measurements on other QD systems [61,145] and will be addressed in detail in the next Chapter. 


\subsection{Emission Spectrum and Photon Statistics}

In this section, we will present the calculated emission spectrum and second-order correlation function $g^{(2)}(\tau)$ of QD1 based on the 3-level model. Experimentally, a high-resolution scanning Fabry-Pérot interferometer (FPI) is needed to measure the sharp emission line structure. The resolution of the spectrometer was not high enough to resolve these structures. At the point of this dissertation, we are building a scanning FPI based on a shearing interferometer in the lab, which would allow us to verify the theoretical results given here by an experimental measurement in the future. For $g^{(2)}(\tau)$, our calculation predicts an oscillatory behavior in photon correlation measurement when the QD is excited at zero-detuning $\left(\omega=\left(\omega_{1}+\omega_{2}\right) / 2\right)$. Unfortunately, this oscillation is too fast for our detectors $(\tau$-SPAD) to resolve.

\subsubsection{Emission spectrum}

A normalized emission spectrum $F\left(\omega_{s c}\right)$ can be obtained via Fourier transforming the first-order correlation function $g^{(1)}(\tau)$ of a system [140],

$$
F\left(\omega_{s c}\right)=\frac{1}{2 \pi} \int_{-\infty}^{\infty} d \tau g^{(1)}(\tau) e^{i \omega_{s c} \tau}
$$

where $\omega_{\text {sc }}$ is the emission angular frequency. Similar to PL intensity given by Eqn. (3.9) and (3.10), $g^{(1)}(\tau)$ has different forms when measured at a specific polarization $\hat{\boldsymbol{\varepsilon}}$ or without any polarization-discrimination

$$
\begin{gathered}
g_{\varepsilon}^{(1)}(\tau)=\frac{\left\langle\left(\hat{\boldsymbol{\varepsilon}} \cdot \boldsymbol{E}^{(-)}(\boldsymbol{r}, t)\right)\left(\widehat{\boldsymbol{\varepsilon}} \cdot \boldsymbol{E}^{(+)}(\boldsymbol{r}, t+\tau)\right)\right\rangle}{\left\langle\left(\widehat{\boldsymbol{\varepsilon}} \cdot \boldsymbol{E}^{(-)}(\boldsymbol{r}, t)\right)\left(\widehat{\boldsymbol{\varepsilon}} \cdot \boldsymbol{E}^{(+)}(\boldsymbol{r}, t)\right)\right\rangle} \\
g^{(1)}(\tau)=\frac{\left\langle\boldsymbol{E}^{(-)}(\boldsymbol{r}, t) \cdot \boldsymbol{E}^{(+)}(\boldsymbol{r}, t+\tau)\right\rangle}{\left\langle\boldsymbol{E}^{(-)}(\boldsymbol{r}, t) \cdot \boldsymbol{E}^{(+)}(\boldsymbol{r}, t)\right\rangle}
\end{gathered}
$$

where $\boldsymbol{E}^{(+)}(\boldsymbol{r}, t)$ is the electric field operator defined in Eqn. (3.8), $\boldsymbol{E}^{(-)}(\boldsymbol{r}, t)$ is the Hermitian adjoint of $\boldsymbol{E}^{(+)}(\boldsymbol{r}, t)$, and $\hat{\boldsymbol{\varepsilon}}$ is the unit vector of the detection polarization. By using Eqn. (3.8) and referring to Figure 3.1(b), Eqn. (3.37) becomes

$$
g^{(1)}(\tau)=\frac{\left\langle\pi_{1}^{\dagger}(t) \pi_{1}(t+\tau)+\pi_{2}^{\dagger}(t) \pi_{2}(t+\tau)\right\rangle}{\left\langle\pi_{1}^{\dagger}(t) \pi_{1}(t)+\pi_{2}^{\dagger}(t) \pi_{2}(t)\right\rangle}
$$

The ensemble average of a single-time operator in the denominator can be evaluated directly by $\left\langle\pi_{j}^{\dagger}(t) \pi_{k}(t)\right\rangle=$ $\tilde{\rho}_{k j}(t)$ (see Section 3.1), while the expectation value of a double-time operator in the numerator, e.g., $\left\langle\pi_{j}^{\dagger}(t) \pi_{k}(t+\tau)\right\rangle$, has to employ the help of quantum regression theory [156-158] and Markov approximation $[140,159]$ to complete the calculation.

The regression theorem states that if the expectation value of any operator $\widehat{A}$ at time $t+\tau$ can be expressed as a sum of the expectation values of a set of operators $\widehat{\mathrm{A}}_{i}$ at an earlier time $t$ as in 


$$
\langle\hat{A}(t+\tau)\rangle=\sum_{i} \alpha_{i}(\tau)\left\langle\hat{A}_{i}(t)\right\rangle
$$

then the expectation value of a double-time operator involving $\widehat{\mathrm{A}}(t+\tau)$ can be evaluated by

$$
\langle\hat{B}(t) \hat{A}(t+\tau) \hat{C}(t)\rangle=\sum_{i} \alpha_{i}(\tau)\left\langle\hat{B}(t) \hat{A}_{i}(t) \hat{C}(t)\right\rangle
$$

Therefore, the two double-time operators in the numerator of $g^{(1)}(\tau)$ can be written as

$$
\begin{aligned}
& \left\langle\pi_{1}^{\dagger}(t) \pi_{1}(t+\tau)\right\rangle=\alpha_{3}(\tau)\left\langle\pi_{1}^{\dagger}(t)\right\rangle+\alpha_{6}(\tau)\left\langle\pi_{1}^{\dagger}(t) \pi_{2}(t)\right\rangle+\alpha_{8}(\tau)\left\langle\pi_{1}^{\dagger}(t) \pi_{1}(t)\right\rangle \\
& \left\langle\pi_{2}^{\dagger}(t) \pi_{2}(t+\tau)\right\rangle=\beta_{3}(\tau)\left\langle\pi_{2}^{\dagger}(t)\right\rangle+\beta_{6}(\tau)\left\langle\pi_{2}^{\dagger}(t) \pi_{2}(t)\right\rangle+\beta_{8}(\tau)\left\langle\pi_{2}^{\dagger}(t) \pi_{1}(t)\right\rangle
\end{aligned}
$$

where coefficients $\alpha_{j}, \beta_{j}$ are the coefficients for operator $\pi_{1}$ and $\pi_{2}$, respectively. These coefficients can be found with help of the Markov approximation, which is valid when the correlations in the environment decay much faster than the time-scale of the evolution in the system of interest. Under this assumption, the memory effects describing how the system has previously interact with environment can be neglected. Consequently, the density matrix $\tilde{\rho}(t)$ in Eqn. (3.7) follows

$$
\tilde{\rho}(t+\tau)=U(\tau) \tilde{\rho}(t)
$$

where $U(t)=V e^{\Lambda t} V^{-1}$ is a 9-by-9 time propagation operator, $\Lambda$ is the diagonalized matrix of supermatrix $\mathcal{M}$ in Eqn. (3.7), and $\mathrm{V}$ is the eigenvector matrix of $\mathcal{M}$. Therefore, Eqn. (3.41) becomes

$$
\begin{aligned}
& \left\langle\pi_{1}^{\dagger}(t) \pi_{1}(t+\tau)\right\rangle=e^{-i \omega \tau}\left\{U_{83}(\tau) \tilde{\rho}_{01}(t)+U_{86}(\tau) \tilde{\rho}_{21}(t)+U_{88}(\tau) \tilde{\rho}_{11}(t)\right\} \\
& \left\langle\pi_{2}^{\dagger}(t) \pi_{2}(t+\tau)\right\rangle=e^{-i \omega \tau}\left\{U_{63}(\tau) \tilde{\rho}_{02}(t)+U_{66}(\tau) \tilde{\rho}_{22}(t)+U_{68}(\tau) \tilde{\rho}_{12}(t)\right\}
\end{aligned}
$$

Note since only $U_{n m}(\tau)$ has $\tau$ dependence, the Fourier transform would only apply to it

$$
Q_{n m}=\frac{1}{2 \pi} \int_{-\infty}^{\infty} d \tau U_{n m}(\tau) e^{i \omega_{s c} \tau}=\frac{1}{\pi} \operatorname{Re} \sum_{k}\left\{V_{n k} \frac{-1}{\Lambda_{k k}+i\left(\omega_{s c}-\omega\right)} V_{k m}^{-1}\right\}
$$

where $\Lambda_{k k}=a_{k}+i b_{k}$ is the eigenvalue of super-matrix $\mathcal{M}$ with both $a_{k}$ and $b_{k}$ as real numbers. Substitute Eqn. (3.43) and (3.44) back to Eqn. (3.38), and the final expressions for the emission spectrum are

$$
F\left(\omega_{s c}\right)=\operatorname{Re}\left\{\frac{Q_{83} \tilde{\rho}_{01}^{\infty}+Q_{86} \tilde{\rho}_{21}^{\infty}+Q_{88} \tilde{\rho}_{11}^{\infty}+Q_{63} \tilde{\rho}_{02}^{\infty}+Q_{66} \tilde{\rho}_{22}^{\infty}+Q_{68} \tilde{\rho}_{12}^{\infty}}{\tilde{\rho}_{11}^{\infty}+\tilde{\rho}_{22}^{\infty}}\right\}
$$

Similarly, for $\mathrm{X}$ - and Y-polarized detections,

$$
\begin{aligned}
& F_{X}\left(\omega_{s c}\right)=\operatorname{Re}\left\{\frac{\left(Q_{88}-Q_{68}\right)\left(\tilde{\rho}_{11}^{\infty}-\tilde{\rho}_{12}^{\infty}\right)+\left(Q_{66}-Q_{86}\right)\left(\tilde{\rho}_{22}^{\infty}-\tilde{\rho}_{21}^{\infty}\right)+\left(Q_{83}-Q_{63}\right)\left(\tilde{\rho}_{01}^{\infty}-\tilde{\rho}_{02}^{\infty}\right)}{\tilde{\rho}_{11}^{\infty}+\tilde{\rho}_{22}^{\infty}-\tilde{\rho}_{21}^{\infty}-\tilde{\rho}_{12}^{\infty}}\right\} \\
& F_{Y}\left(\omega_{s c}\right)=\operatorname{Re}\left\{\frac{\left(Q_{88}+Q_{68}\right)\left(\tilde{\rho}_{11}^{\infty}+\tilde{\rho}_{12}^{\infty}\right)+\left(Q_{66}+Q_{86}\right)\left(\tilde{\rho}_{22}^{\infty}+\tilde{\rho}_{21}^{\infty}\right)+\left(Q_{83}+Q_{63}\right)\left(\tilde{\rho}_{01}^{\infty}+\tilde{\rho}_{02}^{\infty}\right)}{\tilde{\rho}_{11}^{\infty}+\tilde{\rho}_{22}^{\infty}+\tilde{\rho}_{21}^{\infty}+\tilde{\rho}_{12}^{\infty}}\right\}
\end{aligned}
$$

Note Eqns. (3.45) (3.47) give the normalized emission spectra. The denominators of these equations are proportional to the total intensity of the scattered light as given by Eqns. (3.11) (3.13). Hence, the numerators of these equations give the unnormalized emission spectra that are proportional to one single global constant,

$$
\begin{gathered}
S\left(\omega_{s c}\right) \propto 2 \operatorname{Re}\left\{Q_{83} \tilde{\rho}_{01}^{\infty}+Q_{86} \tilde{\rho}_{21}^{\infty}+Q_{88} \tilde{\rho}_{11}^{\infty}+Q_{63} \tilde{\rho}_{02}^{\infty}+Q_{66} \tilde{\rho}_{22}^{\infty}+Q_{68} \tilde{\rho}_{12}^{\infty}\right\} \\
S_{X}\left(\omega_{s c}\right) \propto \operatorname{Re}\left\{\left(Q_{88}-Q_{68}\right)\left(\tilde{\rho}_{11}^{\infty}-\tilde{\rho}_{12}^{\infty}\right)+\left(Q_{66}-Q_{86}\right)\left(\tilde{\rho}_{22}^{\infty}-\tilde{\rho}_{21}^{\infty}\right)+\left(Q_{83}-Q_{63}\right)\left(\tilde{\rho}_{01}^{\infty}-\tilde{\rho}_{02}^{\infty}\right)\right\} \\
S_{Y}\left(\omega_{s c}\right) \propto \operatorname{Re}\left\{\left(Q_{88}+Q_{68}\right)\left(\tilde{\rho}_{11}^{\infty}+\tilde{\rho}_{12}^{\infty}\right)+\left(Q_{66}+Q_{86}\right)\left(\tilde{\rho}_{22}^{\infty}+\tilde{\rho}_{21}^{\infty}\right)+\left(Q_{83}+Q_{63}\right)\left(\tilde{\rho}_{01}^{\infty}+\tilde{\rho}_{02}^{\infty}\right)\right\}
\end{gathered}
$$

Figure 3.11(a) shows the power dependence of the total emission spectra calculated by using Eqn. (48) with QD1 parameters and fixed excitation frequency at zero-detuning, $\omega=\left(\omega_{1}+\omega_{2}\right) / 2$. The delta-function-like sharp peak at $\delta_{\mathrm{sc}}=0$ corresponds to the coherent scattering of the excitation laser; the rest peaks are in Lorentzian line shape, corresponding to the incoherent scattering from the QD. At low excitation power $\left(\Omega_{R} \leq \Gamma_{\mathrm{sp}}\right)$, the 

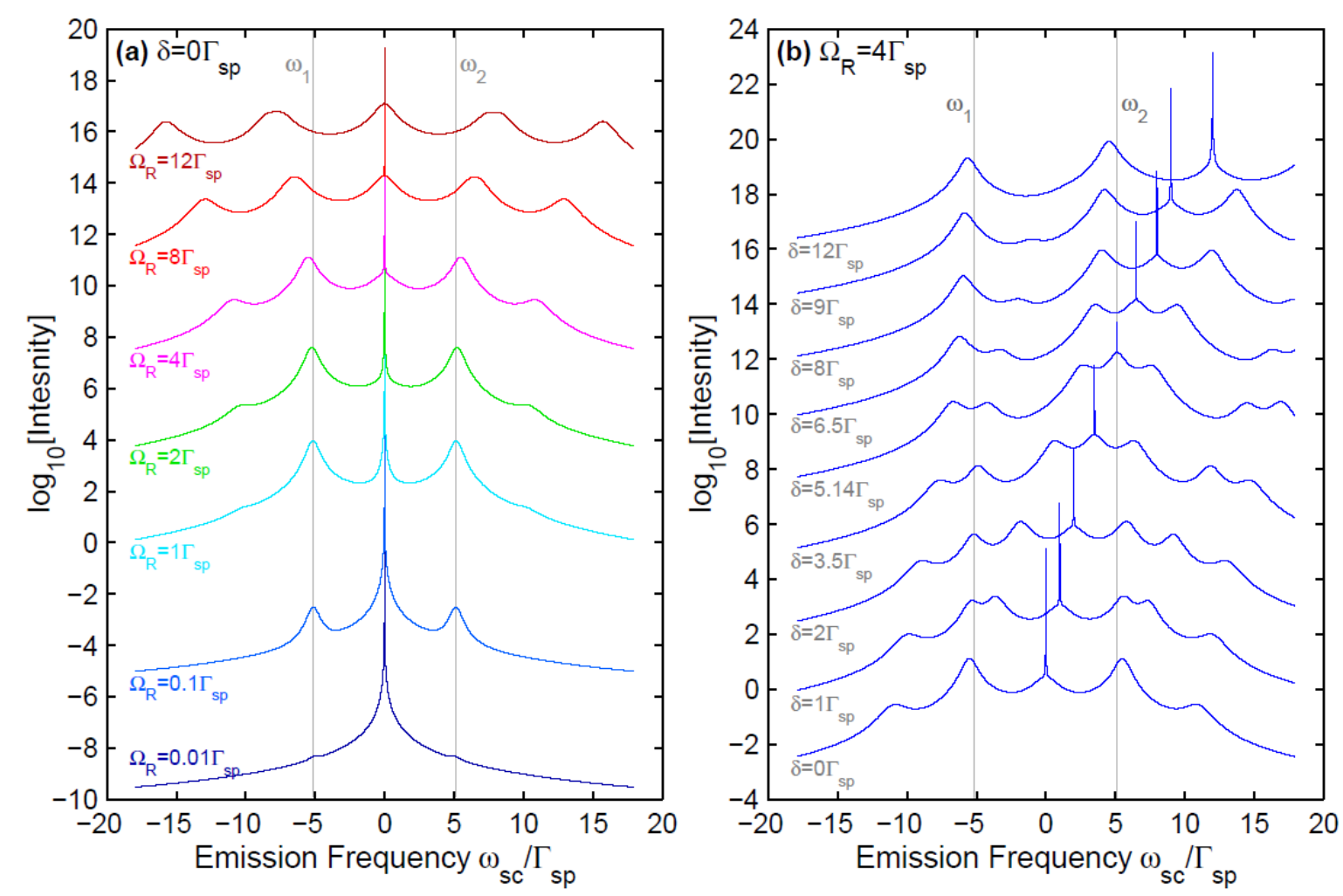

Figure 3.11 Calculated emission spectra of QD1 by tuning (a) excitation power, or (b) excitation wavelength. For (a), the excitation wavelength is fixed at zero-detuning $(\delta=0)$; while for (b), the excitation power is $\Omega_{R}=$ $4 \Gamma_{s p}$. All spectra are plotted in the logarithmic scale and then offset in y-axis for clarity (by adding a constant 2.5 to the each curve above). The two vertical grey lines in (a) and (b) depict the intrinsic transition frequencies of QD1, labeled by $\omega_{1}$ and $\omega_{2}$, respectively. Zero point of both excitation and emission frequency is defined at $\left(\omega_{1}+\omega_{2}\right) / 2$.

excitation photons are incoherently scattered by the two bare eigenstates of the QD (|1 $\rangle$ and $|2\rangle)$ and one "virtual" state at the laser frequency. With increasing excitation power $\left(\Omega_{R} \geq \Gamma_{\mathrm{sp}}\right)$, the energy eigenstates of the QD start to hybridize with the number states of the excitation photons, which results in the formation of the dressed states $[135,160]$. This is evidenced by the appearance of the extra peaks on the low- and high-end of the spectra, and the power-dependent shift of these peaks. This energy structure, called the Mollow quintuplet, has been confirmed experimentally by coherently exciting a neutral QD [161].

As shown in Figure 3.11(b), when the excitation laser is detuned from 0 to $12 \Gamma_{\text {sp }}$ while keeping the excitation power as a constant $\left(\Omega_{\mathrm{R}}=4 \Gamma_{\mathrm{sp}}\right)$, the emission spectrum shows a more complicated behavior. When the QD is excited at zero-detuning $\left(\omega_{L}=\left(\omega_{1}+\omega_{2}\right) / 2\right)$, two pairs of transitions in the dressed state picture are degenerate, leading to a five-peak line structure, as shown by the bottom spectrum in Figure 3.11(b). This line structure has been experimentally confirmed by measuring the emission spectrum of a neutral QD under zero-detuned excitation [161]. In reference [161], however, the authors named this energy structure as "Mollow quintuplet", implying that the authors might miss the point that the emission line is actually composed of seven peaks instead of five peaks in the dressed state picture. It probably worth an effort to verify these lines, especially for various 
excitation wavelengths. In this experiment, a scanning Fabry-Perot interferometer (FPI) is needed to resolve the multiplet structure in an emission spectrum since a typical spectrometer cannot resolve these fine structures. At the time when this dissertation is written, we are building a shearing interferometer based scanning FPI with a finesse of 200 and resolution of $137 \mathrm{MHz}$, which would enable us to complete these measurements when it is set up.

\subsubsection{Second-order correlation function: $g^{(2)}(\tau)$}

The degree of second-order temporal coherence $g^{(2)}(\tau)$ of electromagnetic wave with stationary statistical properties is defined as

$$
g^{(2)}(\tau)=\frac{\langle\bar{I}(t) \bar{I}(t+\tau)\rangle}{\bar{I}^{2}}=\frac{\left\langle\boldsymbol{E}^{\star}(t) \boldsymbol{E}^{\star}(t+\tau) \boldsymbol{E}(t+\tau) \boldsymbol{E}(t)\right\rangle}{\left\langle\boldsymbol{E}^{\star}(t) \boldsymbol{E}(t)\right\rangle^{2}}
$$

where $\bar{I}$ is the long-time average intensity, $\boldsymbol{E}(t)$ is the electric field at the detector at time $t$ and $\boldsymbol{E}^{\star}(t)$ is its complex conjugate counterpart. Physically, $g^{(2)}(\tau)$ measures the relative probability of finding a photon pair with the time separation of $\tau$. For example, for the light source composed of $\mathrm{N}$ identical but independent emitters (e.g., $\mathrm{N}$ atoms), the detected light field would be a sum of the contributions from each individual emitter with a random phase. This means that the summed electric field is coherent only within a finite period of time $\tau_{c}$, called coherence time. When $\tau \gg \tau_{c}$, the time-averaged intensities $\bar{I}(t)$ and $\bar{I}(t+\tau)$ become independent of each other, leading to

$$
\lim _{\tau \rightarrow \infty}\langle\bar{I}(t) \bar{I}(t+\tau)\rangle=\lim _{\tau \rightarrow \infty}\langle\bar{I}(t)\rangle\langle\bar{I}(t+\tau)\rangle=\langle\bar{I}(t)\rangle^{2}
$$

Therefore,

$$
g^{(2)}\left(\tau>\tau_{c}\right) \approx 1
$$

This means that the probability of finding a photon pair separated by any time interval $\tau>\tau_{c}$ is the same, i.e., the photons are completely random-distributed on time scale larger than $\tau_{c}$. However, for $\tau<\tau_{c}$, the distribution of the photons show some features reflecting the properties of the light source. For example, for a source with a Lorentzian or Gaussian emission line shape, $g^{(2)}(\tau)$ has the following form: (Chapter 3.7 of [140])

$$
\begin{aligned}
& g^{(2)}(\tau)=1+e^{-2 \gamma|\tau|} \\
& g^{(2)}(\tau)=1+e^{-\sigma^{2} \tau^{2}}
\end{aligned}
$$

where $\gamma$ is the half-width half-maximum (HWHM) of the Lorentzian distribution in angular frequency, and $\sigma^{2}$ is the variance of the Gaussian distribution. The coherence time $\tau_{c}$ is related to the line-width through $\tau_{c}=$ $1 / \gamma$ and $\tau_{c}=\sqrt{\pi} / \sigma$, respectively. Both $g^{(2)}(\tau)$ follow Eqn. (3.53) for large $\tau$. While for $\tau<\tau_{c}, g^{(2)}(\tau)>$ 1 , specifically $g^{(2)}(0)=2$. This implies that these photons tend to cluster together, i.e., bunching. For coherent photons from a single-mode laser, $g^{(2)}(\tau)=1$ for all $\tau$. Note Eqn. (3.54) or (3.55) is no longer valid for this case. Instead, one can model the coherent light field as a sine wave to reach this conclusion.

All the discussions above are compatible with the classical description of the light, modeled as an electromagnetic wave. However, the light emitted by an isolated individual emitting species (e.g., an individual atom, molecule, quantum dot, or color center) exhibits antibunched correlation statistics $\left(g^{(2)}(0)<1\right)$ [162], which is a purely quantum optical phenomenon with no classical counterpart. In antibunched light, the photons come out 
with regular gaps rather than with a random spacing [163]. Theoretically, this can be described by replacing the classical field in Eqn. (3.51) with the electric field operator via quantization of the electromagnetic waves,

$$
g^{(2)}(\tau)=\frac{\left\langle\boldsymbol{E}^{(-)}(t) \boldsymbol{E}^{(-)}(t+\tau) \boldsymbol{E}^{(+)}(t+\tau) \boldsymbol{E}^{(+)}(t)\right\rangle}{\left\langle\boldsymbol{E}^{(-)}(\boldsymbol{r}, t) \boldsymbol{E}^{(+)}(\boldsymbol{r}, t)\right\rangle^{2}}
$$

where $\boldsymbol{E}^{(+)}(t)$ is the electric field operator defined in Eqn. (8) for a neutral QD, and $\boldsymbol{E}^{(-)}(\boldsymbol{r}, t)$ is the Hermitian adjoint of $\boldsymbol{E}^{(+)}(t)$. For a specific polarization measurement along $\hat{\boldsymbol{\varepsilon}}$,

$$
g_{\varepsilon}^{(2)}(\tau)=\frac{\left\langle\left(\hat{\boldsymbol{\varepsilon}} \cdot \boldsymbol{E}_{s c}^{(-)}(\boldsymbol{r}, t)\right)\left(\hat{\boldsymbol{\varepsilon}} \cdot \boldsymbol{E}_{s c}^{(-)}(\boldsymbol{r}, t+\tau)\right)\left(\hat{\boldsymbol{\varepsilon}} \cdot \boldsymbol{E}_{s c}^{(+)}(\boldsymbol{r}, t+\tau)\right)\left(\widehat{\boldsymbol{\varepsilon}} \cdot \boldsymbol{E}_{s c}^{(+)}(\boldsymbol{r}, t)\right)\right\rangle}{\left\langle\left(\hat{\boldsymbol{\varepsilon}} \cdot \boldsymbol{E}_{s c}^{(-)}(\boldsymbol{r}, t)\right)\left(\hat{\boldsymbol{\varepsilon}} \cdot \boldsymbol{E}_{s c}^{(+)}(\boldsymbol{r}, t)\right)\right\rangle^{2}}
$$

For X-polarization,

$$
g_{X}^{(2)}(\tau)=\frac{\left\langle\left[\pi_{1}^{\dagger}(t)-\pi_{2}^{\dagger}(t)\right]\left[\pi_{1}^{\dagger}(t+\tau)-\pi_{2}^{\dagger}(t+\tau)\right]\left[\pi_{1}(t+\tau)-\pi_{2}(t+\tau)\right]\left[\pi_{1}(t)-\pi_{2}(t)\right]\right\rangle}{\left\langle\pi_{1}^{\dagger}(t) \pi_{1}(t)-\pi_{2}^{\dagger}(t) \pi_{1}(t)-\pi_{1}^{\dagger}(t) \pi_{2}(t)+\pi_{2}^{\dagger}(t) \pi_{2}(t)\right\rangle^{2}}
$$

where we have used Eqn. (3.8) to substitute the electric field operators. With the help of quantum regression theory and the Markov approximation (similar method used in Section 3.3.1 to derive Eqn. (3.41) and (3.43)), we can calculate the double time operators in the numerator of Eqn. (3.58) as following

$$
\begin{aligned}
& \left\langle\pi_{i}^{\dagger}(t) \pi_{1}^{\dagger}(t+\tau) \pi_{1}(t+\tau) \pi_{k}(t)\right\rangle=U_{23}(\tau) \tilde{\rho}_{k i}(t) \\
& \left\langle\pi_{i}^{\dagger}(t) \pi_{2}^{\dagger}(t+\tau) \pi_{1}(t+\tau) \pi_{k}(t)\right\rangle=U_{53}(\tau) \tilde{\rho}_{k i}(t) \\
& \left\langle\pi_{i}^{\dagger}(t) \pi_{1}^{\dagger}(t+\tau) \pi_{2}(t+\tau) \pi_{k}(t)\right\rangle=U_{43}(\tau) \tilde{\rho}_{k i}(t) \\
& \left\langle\pi_{i}^{\dagger}(t) \pi_{2}^{\dagger}(t+\tau) \pi_{2}(t+\tau) \pi_{k}(t)\right\rangle=U_{13}(\tau) \tilde{\rho}_{k i}(t)
\end{aligned}
$$

where $U_{n m}(\tau)$ is an element of the time-propagation operator in Eqn. (3.42). With these relations, we reach the final expressions of second-order coherence for either X-, Y- or no polarization,
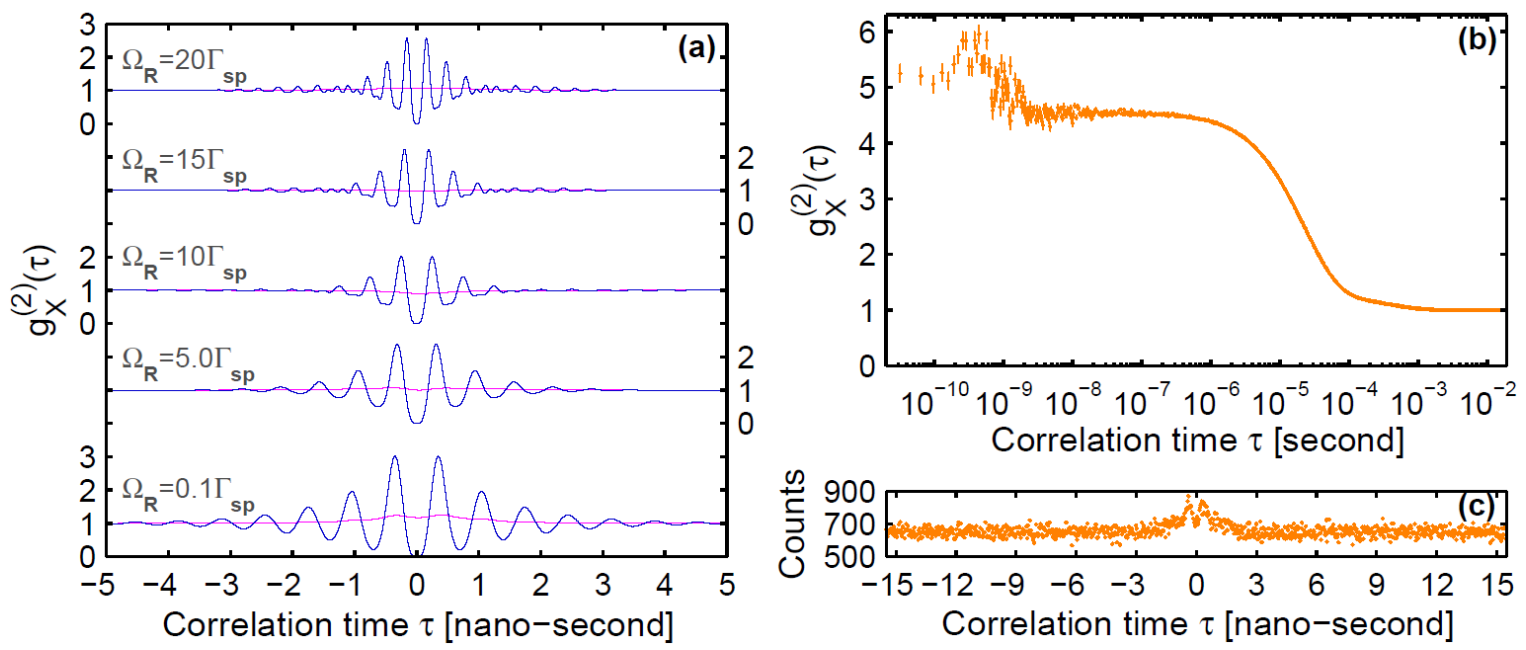

Figure 3.12 (a) Calculated $g_{X}^{(2)}(\tau)$ from QD1 at different excitation powers. The excitation wavelength is fixed at zero-detuning. The magenta curves are the convolved results of blue curves with the measured instrument response function given in the inset of Figure 2.6(d). (b) Experimentally measured $g_{X}^{(2)}(\tau)$ from QD1, excited at zero-detuning and with a power of $\Omega_{R}=1.31 \Gamma_{s p}$. The correlation time is in logarithmic time scale for (b), and in linear scale for (c). 


$$
\begin{gathered}
g_{X}^{(2)}(\tau)=\frac{U_{13}(\tau)+U_{23}(\tau)-U_{43}(\tau)-U_{53}(\tau)}{\tilde{\rho}_{22}^{\infty}+\tilde{\rho}_{11}^{\infty}-2 \operatorname{Re}\left[\tilde{\rho}_{21}^{\infty}\right]} \\
g_{Y}^{(2)}(\tau)=\frac{U_{13}(\tau)+U_{23}(\tau)+U_{43}(\tau)+U_{53}(\tau)}{\tilde{\rho}_{22}^{\infty}+\tilde{\rho}_{11}^{\infty}+2 \operatorname{Re}\left[\tilde{\rho}_{21}^{\infty}\right]} \\
g^{(2)}(\tau)=\frac{U_{13}(\tau)+U_{23}(\tau)}{\tilde{\rho}_{22}^{\infty}+\tilde{\rho}_{11}^{\infty}}
\end{gathered}
$$

where $\tilde{\rho}_{j k}^{\infty}$ are the steady-state solution of density matrix considering the long exposure time.

Figure 3.12(a) shows the calculated $g_{X}^{(2)}(\tau)$ from QD1 excited at zero-detuning with different powers. Blue curves are the calculated results from Eqn. (3.60), showing strong oscillations. Magenta curves are the results considering the finite response time of our detectors ( $\tau$-SPAD), i.e., convolving the blue curves with the instrument response function (IRF) given in the inset of Figure 2.6(d). The fast oscillations $(>2 \mathrm{GHz})$ are significantly smoothed out by the relatively slow response $(\sim 0.9 \mathrm{~ns})$ of our detectors. The residual oscillation, barely observable in lowest-power excitation, is probably too weak to be resolvable from the noise, especially when a strong photon bunching is present in experimental data, as shown in Figure 3.12(b-c). In this measurement, QD1 was excited at zero-detuning and with an excitation power of $\Omega_{\mathrm{R}}=1.31 \Gamma_{\mathrm{sp}}$. Even though long integration time ( 69 hours) has been implemented, aiming for decreasing the noise, it is still not convincing that a similar oscillation is present in Figure 3.12(b-c). But the existence of strong photon bunching implies that the QD emission is disturbed by some other physical processes, probably related to the charge environment fluctuation triggered by the excitation laser. This phenomenon will be addressed in detail in the next chapter. 


\subsection{Conclusion}

In this study, we have demonstrated and modeled an interference effect that occurs during $c w$ resonant excitation of a multi-level quantum system. Including coherent scattering is necessary to explain the strong polarization difference between the excitation and the emission. Such a phenomenon does not occur under incoherent or pulsed excitation. Comparison of spectra with different detection polarizations allows extraction of the steadystate coherence generated between the two excited states. All the spectra and coherences are correctly reproduced by a density-matrix model of the QD. Similar effects must be accounted for in any situation where there are two non-degenerate orthogonal transition dipole moments and only a certain polarization is detected. One example is the "dark-field" resonant excitation and detection technique [123] in combination with a charged QD in an inplane magnetic field. 


\section{Chapter 4: Local Electric Environment Fluctuation}

Summary. The subtle fluctuations of the local electric field environment would modify the energy levels of the quantum dot (QD) through the quantum-confined Stark effect. This random energy fluctuation impairs the indistinguishability between the photons emitted by a single QD, which in further restrains the QD's potential in quantum technology applications. The first step to overcome this barrier is to characterize and quantify the influence on the QD through experimental measurements, and extract the insight via physical modeling. Spectroscopically, this effect manifests as a discrete jump, continuous drift or spectral broadening of the QD's absorption and emission lines depending on the relative position of the charge traps and characteristic time of the electric fluctuations. The study in this chapter will show that all of these effects are present in one QD's (labeled as QD2) excitation spectra when its local environment is perturbed by above band-gap optical excitation. By analyzing the discrete spectral jumps, we are able to determine the number, relative position, and average occupation of the nearby charge traps. Second-order correlation measurements of the emitted fluorescence show photon bunching that characterizes the time scale of the charge trap dynamics. We found that the switching rate of the charge traps between occupied and unoccupied configurations increases by five orders of magnitude with increasing above band-gap excitation power even below the threshold where the above-band excitation produces photoluminescence (PL) on its own. 


\subsection{Stark Effect}

For an InGaAs QD, the fluctuation of the local electric field is usually caused by the charge dynamics of the charge traps that are located not too far from the QD. These dynamics often take place at a rate between $\mathrm{kHz}$ and $\mathrm{MHz}$, depending on the intrinsic properties (size and depth) of the trap and the density of the local free charge carriers. Comparing to the recombination rate of the neutral exciton in a $\mathrm{QD}(\sim 1 \mathrm{~ns})$, this is a very slow process that justifies the quasi-static feature of the local electric field with respect to the QD emission. Therefore, we can formulate the QD-field interaction within the framework of classical theory, i.e., simply dot product of the electric dipole moment of the QD with the local electric field. By including the field induced dipole moment, the final frequency shift caused by this extra interaction energy is

$$
\Delta v=-(\boldsymbol{p}+\boldsymbol{\beta} \cdot \boldsymbol{F}) \cdot \boldsymbol{F} / h
$$

where $\Delta v$ is the Stark shift of the resonance frequency of the neutral exciton when a local electric field $\boldsymbol{F}$ is present, $\boldsymbol{p}$ is the permanent static dipole moment of the confined exciton, and $\boldsymbol{\beta}$ is its polarizability tensor. Thus, a charge trap close to a QD will result in a discrete shift of the resonance peak in the QD's absorption spectrum when the trap's occupation state changes. A single trap far from a QD will have an unnoticeable effect on the QD by itself, but a change in mean occupation of a large ensemble of traps will cause a continuous drift or broadening of the QD spectrum.

In InGaAs QDs, the electron and hole wave functions is separated mostly along growth axis with a separation of a few sub nanometers $[99,100,103,164]$, thus $\boldsymbol{p}=p \hat{\mathrm{z}}=e r \hat{\mathrm{z}}$, where the sign of $p$ is determined by the amount of strain experienced by the QD, as discussed in Section 2.2.1. The polarizability tensor $\boldsymbol{\beta}$ is also anisotropic considering the aspherical shape of the dot. Given the QD's pancake-like shape, it has negligible polarizability in the z-direction, $\beta_{z z}=0$. Thereby, Eqn. (4.1) can be rewritten as

$$
\Delta v=-\left[p \cdot F_{z}+\beta\left(F_{x}^{2}+F_{y}^{2}\right)\right] / h
$$

where $\beta=\beta_{\mathrm{xx}}=\beta_{\mathrm{yy}}$ is in-plane polarizability of the QD. For applications of quantum dots as emitters and detectors, the polarizability should be large so that the energy can be tuned over a large range with modest electric fields. Conversely, for applications involving coherent control or high-resolution spectroscopy, the polarizability should be small because the Stark effect contributes to the line width, in particular of single dot emission, through the local electric fields generated by fluctuating charge [103].

The electric field $\boldsymbol{F}$ at the dot location is governed by Coulomb's law

$$
\boldsymbol{F}=\sum_{k} \frac{1}{4 \pi \epsilon_{0} \epsilon_{r}} \frac{-q_{k}}{r_{k}^{2}} \hat{\mathbf{r}}_{k}
$$

where $q_{k}$ is the charge in trap $k, \boldsymbol{r}_{k}$ is the relative position of trap $k$ with respect to the dot, and $\epsilon_{r}$ is the relative dielectric constant of the surrounding medium, i.e., GaAs in this case. In principle, the index $k$ should go over all the charge traps in the sample, but most traps are located far from the dot that barely have a notable impact on QD, and thus can be neglected. Only the nearby traps need to be counted. For $\mathrm{n}$ independent nearby traps, there would be $N$ charge configurations 


$$
N=\prod_{k=1}^{n} N_{k}
$$

where $N_{k}$ is the number charge states for trap $k$. If the resultant $N$ electric fields (each from one configuration) are significant different from each other, we expect to observe $N$ discrete spectral shifts in excitation and emission spectrum. 


\subsection{Experiments}

The sample consists of InGaAs self-assembled QDs grown in the center of a 4- $\lambda$ GaAs spacer between two AlAs/GaAs distributed Bragg reflectors (DBRs) as shown in Figure 2.1(d). The DBRs form a planar microcavity with a transmission mode from $923 \sim 930 \mathrm{~nm}$ and a waveguide mode that confines light within the plane of the sample. The thickness of the GaAs spacer that defines the cavity is $1.06 \mu \mathrm{m}$, leaving the QDs more than $500 \mathrm{~nm}$ away from either DBR. As discussed later, this relatively large distance rules out the possibility that the observed discrete Stark shifts in the RPLE data are caused by charging of defects at the hetero-interfaces or the sample surface. The experimental method has been detailed in Section 2.3 with the optical setup shown in Figure 2.6. In this study (this chapter), only two detection polarizations are used, horizontal (X) and vertical (Y). The sample is always maintained at $4.2 \mathrm{~K}$ through all measurements. As mentioned earlier, the dot addressed here is QD2, a different QD from QD1 that is studied in Chapter 3. Both QDs are located approximately $100 \mu \mathrm{m}$ from the cleaved edge of the sample. The continuous-wave (cw) laser couples into the sample via waveguide mode of the microcavity from the side, while the photoluminescence (PL) couples out via Fabry-Perot mode in surface normal direction (see Figure 2.6). For lifetime measurement, the $\mathrm{cw}$ laser is replaced by a mode-locked Ti:sapphire laser, to excite QD2 with 2.1 ps long pulses at a repetition rate of $78 \mathrm{MHz}$. An above bandgap

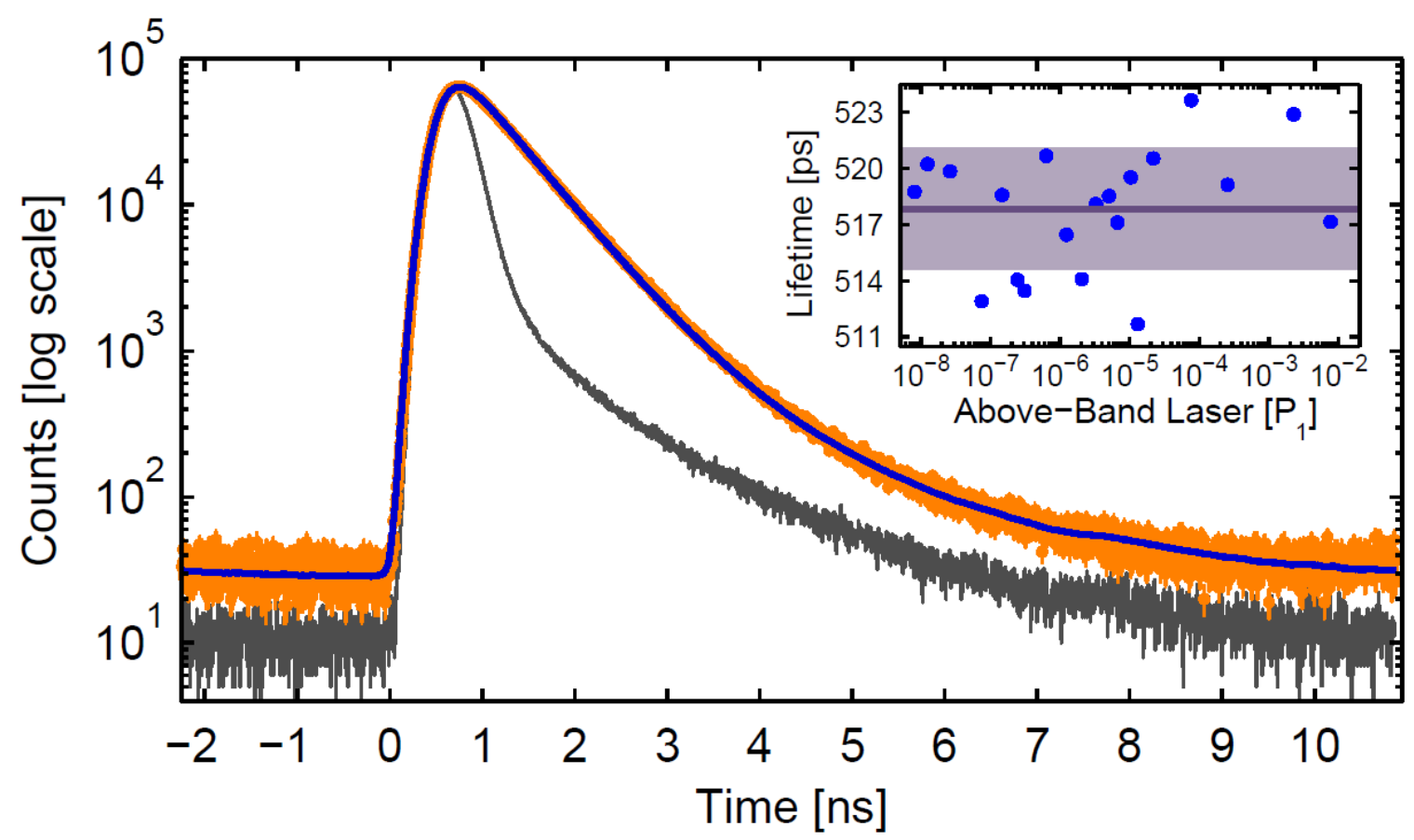

Figure 4.1 Time-resolved fluorescence from a single QD under pulsed resonant excitation and without aboveband excitation. The data (orange dots) are fit by an exponential decay model convolved with the measured instrument response function of the SPCM (black curve). The blue curve is the convolved result. The inset shows the lifetimes extracted from similar time-resolved fluorescence measurements with different levels of above-band laser power represented as a fraction of the saturation power $\mathrm{P}_{1}=28.5 \mu \mathrm{W}$. The lifetime varies little with above-band laser power, with an average value of $T_{1}=518 \pm 3 \mathrm{ps}$. The average $T_{1}$ is depicted by the dark line in the inset, while the gray area is the standard uncertainty range. 
excitation laser (HeNe at $632 \mathrm{~nm}$ ) can also be introduced to the sample via PL collection path. The collected fluorescence from the sample is either recorded by a TE-cooled CCD camera or counted by a time-correlated single-photon counting (TCSPC) system. The second-order correlation function, $g^{(2)}(\tau)$, of the cw-excited fluorescence is calculated from the time-tagged photon detection data.

\subsubsection{Time-resolved fluorescence}

Similar to the case of QD1, the lifetime $\mathrm{T}_{1}$ of QD2 is also determined by time-resolved fluorescence experiment where the dot is resonantly excited by a train of pulses with an energy of $0.17 \mathrm{pJ} / \mathrm{pulse}$. The bandwidth of each pulse is $76 \mathrm{GHz}$ in linear frequency, which is two orders of magnitude wider than QD2's ground state transition linewidth, and thus well covers the observed spectral shifts due to the charge traps. Figure 4.1 shows an example time-resolved measurement without above-band excitation. The data are fitted with an exponential decay convolved with the measured instrument response function of the single photon counting module (grey curve). We measured resonantly excited time-resolved fluorescence for different powers of above-band excitation and extracted an average lifetime of $\mathrm{T}_{1}=(518 \pm 3) \mathrm{ps}$, close to the value of QD1. The extracted lifetimes show no trend with increasing above-band excitation power (inset of Figure 4.1). Along with additional measurements discussed below, this suggests that the lifetime is not affected by the fluctuations of the local charge environment.

\subsubsection{Resonant photoluminescence excitation spectroscopy}

We use resonant photoluminescence excitation (RPLE) spectroscopy to quantify the discrete shifts and continuous drifts of the QD energy levels caused by both nearby and distant charge carrier traps. The capture rate of charge carriers at these traps is expected to depend on the local free charge carrier density, which is controlled by adjusting the power of an above band-gap laser through six orders of magnitude. To enable comparisons with other studies, both resonant and above-band excitation powers are labeled in the unit of saturation power, $\mathrm{P}_{0}=4.7 \mu \mathrm{W}$ and $\mathrm{P}_{1}=28.5 \mu \mathrm{W}$, respectively, determined by fitting the measured PL from $\mathrm{QD} 2\left(I_{\mathrm{PL}}\right)$ at different excitation powers with to the following formula,

$$
I_{\mathrm{PL}}=\mathrm{A} \frac{1}{2} \frac{\mathrm{P}}{\mathrm{P}+\mathrm{P}_{\mathrm{S}}}+k \cdot \mathrm{P}
$$

where $\mathrm{P}$ is the excitation power, $\mathrm{P}_{\mathrm{s}}$ is the saturation power (i.e., $\mathrm{P}_{0}$ or $\mathrm{P}_{1}$ ), $A$ is the intensity constant, and $k$ is a slope parameter. The fitting results are shown in Figure 4.2. The first term in Eqn. (4.5) gives the fluorescence intensity emitted by the dot, which can be derived by modeling the QD as a 2-level system for resonant excitation [140], or by modeling the charge dynamics of the QD with a rate model for above-band excitation (see Appendix A4.1). The second term describes the background signal with a linear power dependence, originated from the laser scattering for resonant excitation, and the emission from the wetting layer defect states for above-band excitation. 

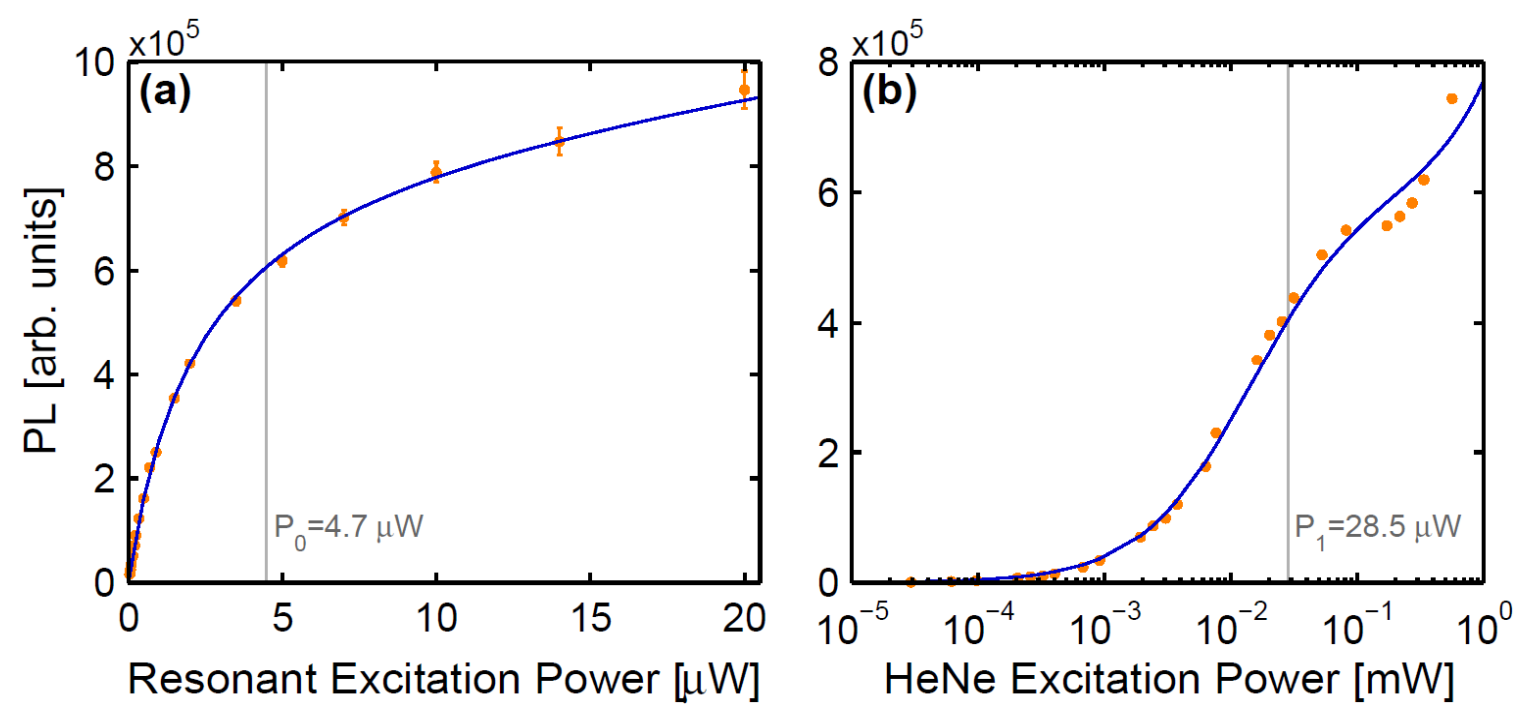

Figure 4.2 The excitation power-dependence of the PL from QD2 when it is excited (a) with a resonant laser, or (b) with an above-band laser. The orange dots are the raw data. The blue curves are the fitting with Eqn. (4.5). The vertical grey lines indicates the saturation power obtained from the fitting, i.e., $\mathrm{P}_{0}=4.7 \mu \mathrm{W}$ for pure resonant excitation and $\mathrm{P}_{1}=28.5 \mu \mathrm{W}$ for above-band excitation. In (a), the cw laser is tuned to be resonance with the high-energy state of QD2; in (b), the power of the above-band laser (at $632 \mathrm{~nm}$ ) is tuned through 5 orders of magnitude, and plotted in logarithmic scale.

For each above-band power, two RPLE spectra with orthogonal detection polarizations (H and V) are collected by scanning the frequency of a tunable $1 \mathrm{MHz}$ bandwidth cw laser through QD2's resonance at a power of $10 \%$ saturation power, i.e., $\mathrm{P}_{\mathrm{res}}=0.1 \mathrm{P}_{0}$. The two spectra are then summed to give a final spectrum whose amplitude is proportional to the exciton population in the QD. In the summation, the polarization-dependent absorption caused by the optics in the collection path is corrected.

Figure 4.3(a-c) show three examples of summed spectra in three different power regimes of the above-band laser, i.e. low, medium, and high power. Each spectrum is fitted with eight Voigt profile peaks whose Lorentzian linewidth is restricted to be not less than the lifetime-limited value of $308 \mathrm{MHz}$ in linear frequency according to $\mathrm{T}_{1}$. It is worth mentioning that the fitting with multiple Lorentzian functions cannot reproduce the data as well as using Voigt functions, and the fact that the environmental fluctuations introduce inhomogeneous broadening to the QD's energy states justifies the Voigt shape of these excitation lines [53,55,123,165-167]. Polarization dependent RPLE [Figure 4.3(e)] suggests that these emission lines are from a neutral QD. Thus eight Voigt peaks gives four pairs corresponding to four different discrete Stark shifts (including the case where the shift is zero) experienced by the QD during the measurement.

We measured RPLE spectra for a number of QDs, and all of them showed either 1, 2, or 4 different Stark shifts. Some examples are given in Figure 4.4(a). These are all powers of 2, which is consistent with an integer number of nearby 2-level charge traps, i.e., $N_{k}=2$ in Eqn. (4.4). None of the QDs we surveyed showed three or five Stark shifts. Regarding the QD detailed here, to match the four discrete shifts observed in the RPLE data, the only possible trap arrangement is either two 2-level traps or one 4-level trap. Candidates for 2-level traps 
abound - for example, dopant impurities and other crystal defects - while to the authors' knowledge no 4-level traps have been reported in the literature. Thus we discard the latter possibility and conclude that there are two nearby 2-level charge traps influencing QD2. We denote the first trap as $\alpha$, the second as $\beta$, and the possible trap configurations as $(\alpha \beta)$, where $\alpha, \beta$ can take a value of 0 or 1 representing the empty (neutral) or occupied (ionized) state of each trap.

In Figure 4.3(a-c), the eight Voigt peaks are color coded into four pairs so that each pair stands for one trap configuration: the green peaks correspond to charge configuration (00), black peaks to configuration (01), blue peaks to configuration (10), and red peaks to configuration (11). Below we discuss the underlying reasoning for
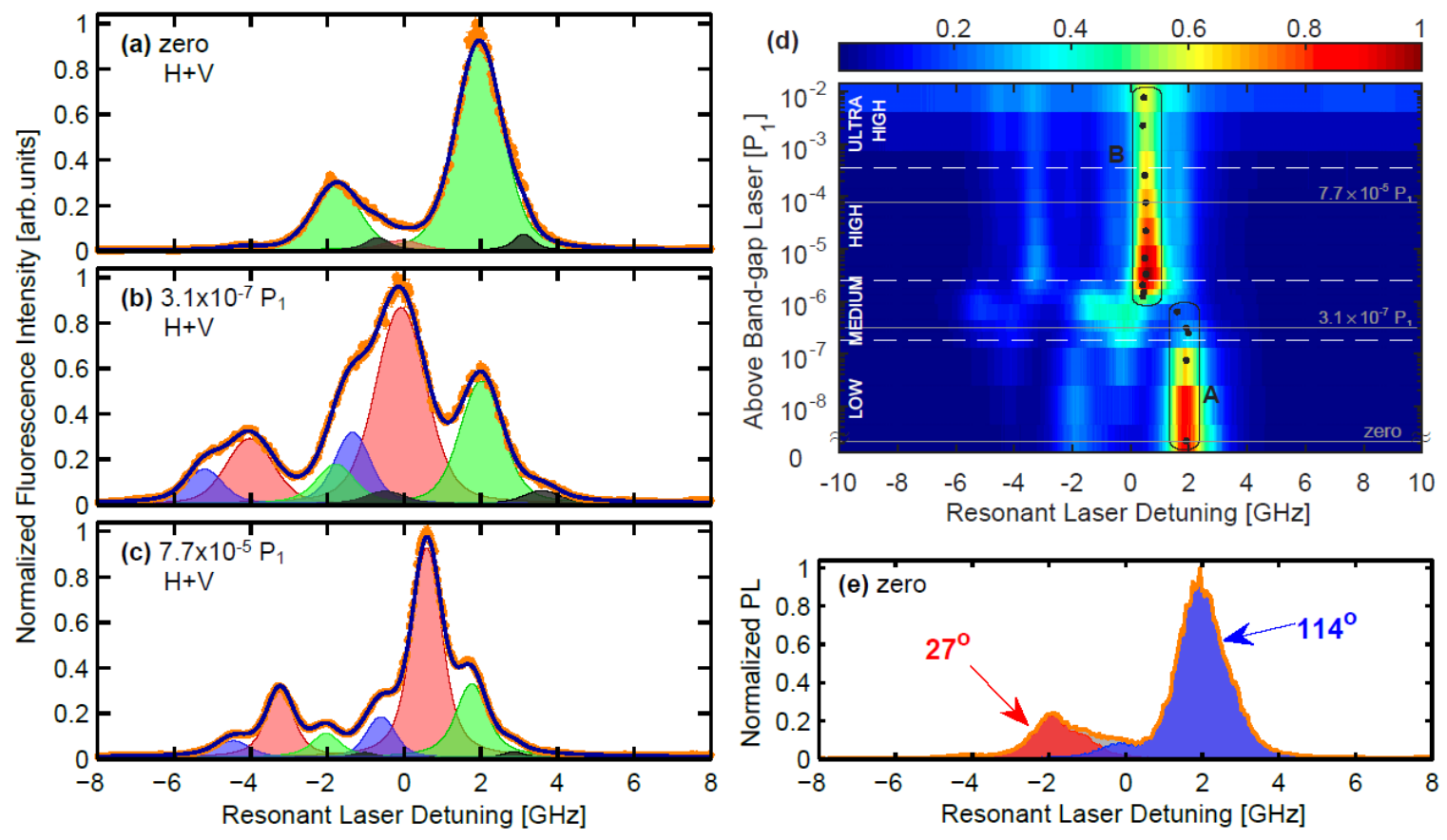

Figure 4.3 (a)-(c) Three examples of normalized resonant photoluminescence excitation spectra (RPLE) at above-band laser powers of zero, $3.1 \times 10^{-7} \mathrm{P}_{1}$ and $7.7 \times 10^{-5} \mathrm{P}_{1}$, respectively. The filled curves are the individual Voigt peaks used to do the fitting; the blue curve along the orange data points is the sum of these individual peaks. Zero detuning is defined as the middle point of the two Voigt peaks with largest amplitude (green) in (a), which corresponds to $928.3713 \mathrm{~nm}$. Each curve is normalized to its own maximum. (d) 2D plot of 16 RPLE spectra taken at different above-band laser powers plotted in a logarithmic scale on the vertical axis. The color-scaled spectral intensity is normalized to the overall maximum of the measured fluorescence intensity. The grey lines denote the three spectra in (a), (b), and (c). The black dots are the positions where correlation data are collected. Box A denotes the data shown in Figure 4.6(c) and box B those in Figure 4.6(d). The white dashed lines indicate the boundaries for the different above-band power regimes. (e) An example of polarization-dependent RPLE without above-band laser. Two RPLE spectra were recorded using a linear polarizer oriented at $114^{\circ}$ (blue) and $27^{\circ}$ (red) from the horizontal. Their sum is displayed as the orange curve with grey filled area. The red and blue peaks are the same shape but displaced and with different amplitudes, which implies that the two peaks are the orthogonally polarized emission from the two fine structure split states of a neutral QD. 
the assignment of these labels. There may be additional charge carrier traps far from the QD and randomly distributed, but their influences on the QD energies are limited. Their effect on the RPLE spectra is described by spectral line broadening, which consists of two parts: Gaussian widths of the Voigt profiles for inhomogeneous environmental broadening and additional Lorentzian widths for homogeneous nonradiative broadening. Due to the significant overlapping of multiple peaks in the spectra, the fitting is too ambiguous to distinguish these two sources definitively. But the Voigt line widths of all peaks are broadened by about a factor of 4 compared to the lifetime-limited value. Considering the relatively weak effect on the electric field at the QD from the free charge carriers and distant charge traps, the orientation of the asymmetry axis of the confinement potential of the QD will remain the same for all above-band powers. Therefore the amplitude ratio of the two fine structure peaks in each pair of Voigt profiles is constant for all above-band powers. We perform a global fitting of four spectra from different power regimes simultaneously to determine the ratios. Afterwards, each spectrum is fitted individually with the fixed amplitude ratios.

With no above-band excitation, one pair of peaks dominates the RPLE spectrum [the green peaks in Figure 4.3(a)] showing that $83 \%$ of the time the traps are in the corresponding configuration. Without above-band excitation,

(a)
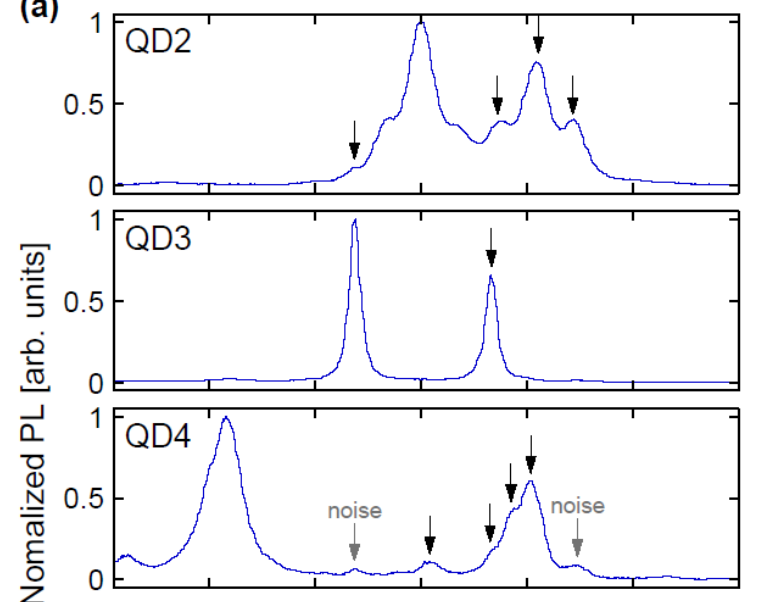

Z

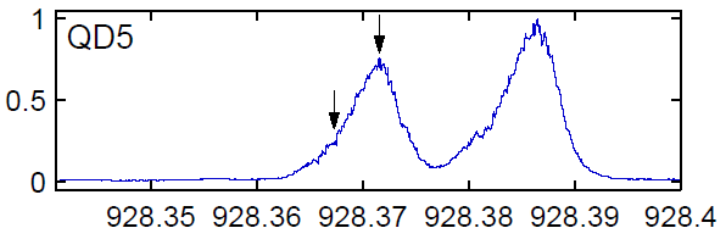

Excitation Wavelength $\lambda[\mathrm{nm}]$ (b)

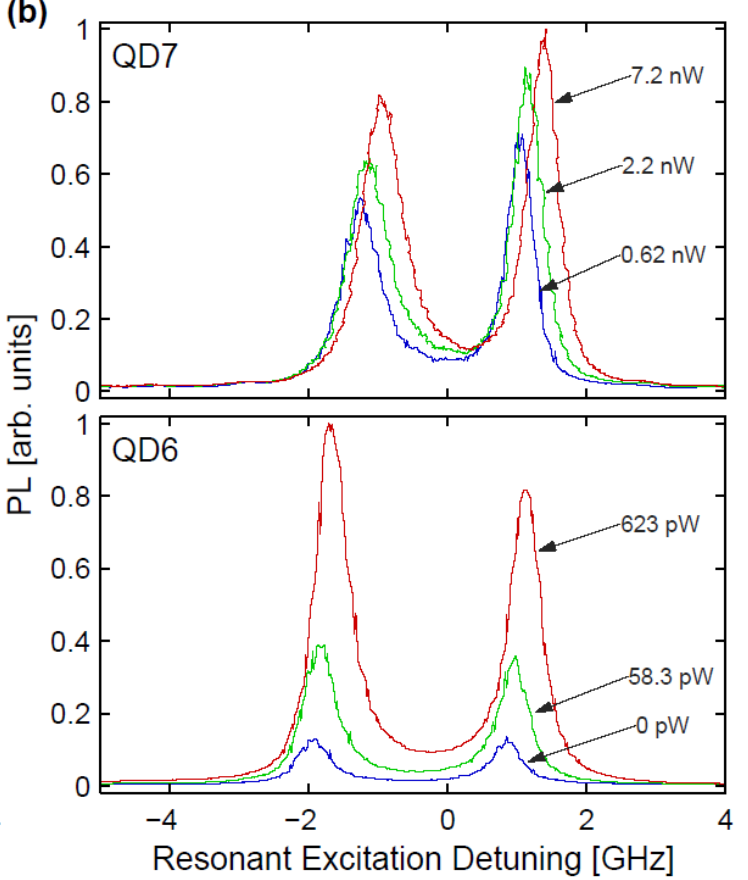

Figure 4.4 (a) RPLE spectra of other three QDs (QD3, QD4 and QD5). RPLE spectra of QD2 is shown on the top as a comparison. All spectra show $2^{n}$ Stark shifts, with $n=2,0,2$ and 1 from top to bottom. The peaks pointed by black arrows correspond to the Stark shifts. The grey arrows in QD4 point at two small humps, which are not the PL from QD4 but the PL ring structure of nearby QDs. We confirm this by checking each frame of RPLE data. (b) Continuous shift of the resonance energy of QD6 and QD7 as increasing the aboveband laser power. Both QDs show blue shift. The power of the above-band laser used to disturb the local environment is labeled by the annotation on the right of each RPLE spectrum. Zero excitation detuning correspond to $927.86 \mathrm{~nm}$ and $928.491 \mathrm{~nm}$ for QD6 and QD7, respectively. 

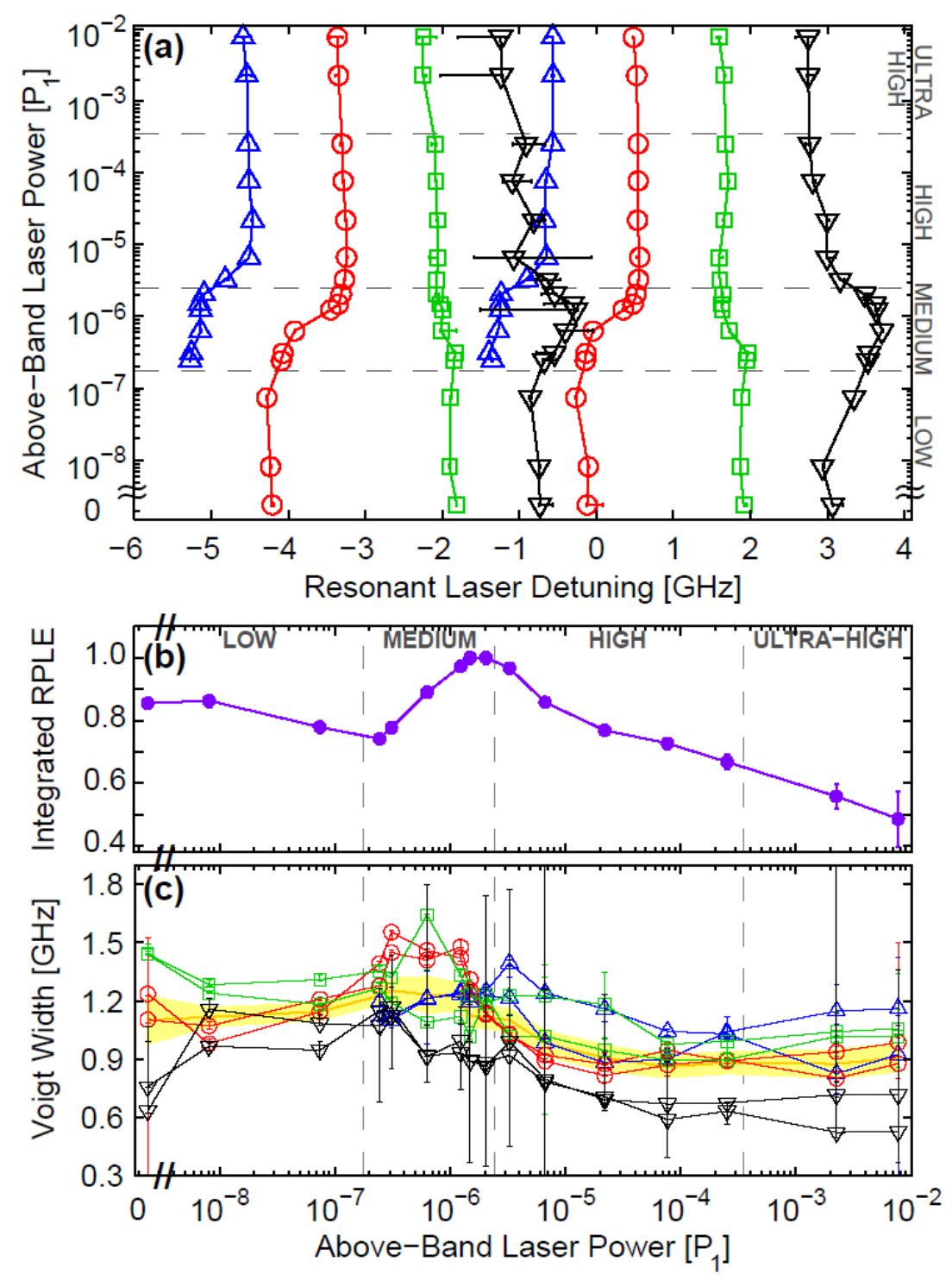

Figure 4.5 (a) Center detunings of resonance peaks. For the RPLE spectra that comprise Figure 4.3(d), the center detuning of each Voigt profile in the fit is plotted vs the corresponding above-band laser power. The green square curve corresponds to trap configuration (00), the red circle for trap configuration (11), the blue up-triangle for trap configuration (10) and black down-triangle for trap configuration (01). The lines are guides for the eye. The horizontal dashed lines give the boundaries of different above-band power regimes. (b) Spectrally integrated intensity of RPLE spectra in Fig. 3(d). The baseline offset due to the above-band excitation has been subtracted so that the curve represents the emission solely due to resonant excitation; this effect is only significant in the ultrahigh-power regime. The error bars correspond to experimental fluctuation and shot noise. The curve is normalized to its maximum value. The grey vertical dashed lines correspond to the boundaries of different above-band power regimes. (c) Voigt line width of each resonance peaks in linear frequency. The choice of color and marker for each peak follows exactly the same as the one adopted in (a). The shaded (yellow) region represents the average and standard deviation of these 8 peak widths. 
the traps are expected to be in thermal equilibrium. If the traps are either shallow donor or acceptor impurities, in bulk GaAs at $4.2 \mathrm{~K}$ the fraction that are ionized is approximately zero. The fraction of ionized dopants near the QD may be increased by the proximity of the wetting layer quasi-continuum states, but it is still expected to be low. Therefore we assign the neutral trap configuration (00) to the green peaks dominating the spectrum in Figure 4.3(a).

At high above-band laser power we expect the free charge carrier density to be greater, and the charge traps correspondingly more likely to be charged compared to the case of low above-band power. Therefore we assign trap configuration (11) to the pair of peaks that dominates the spectrum in Figure 4.3(c), which is displayed in red. The other two configurations (01) and (10) are arbitrarily assigned to the black peaks which are shifted by about $1 \mathrm{GHz}$ from the green, and to the blue peaks which are shifted by about $-3 \mathrm{GHz}$ from the green.

Figure 4.3(d) shows all 16 RPLE spectra at different above-band laser powers which span 6 orders of magnitude. We identify four regimes of above-band power based on the different patterns in the RPLE spectra: low-power regime (zero $\left.\sim 1.7 \times 10^{-7} \mathrm{P}_{1}\right)$, medium-power regime $\left(0.17 \sim 2.5 \times 10^{-6} \mathrm{P}_{1}\right)$, high-power regime $(0.025 \sim$ $3.5 \times 10^{-4} \mathrm{P}_{1}$ ), and ultrahigh-power regime (above $3.5 \times 10^{-4} \mathrm{P}_{1}$ ). Note that even in the high end of the ultrahigh-power regime the above-band laser is less than $1 \%$ of the saturation power.

Figure 4.5(a) shows the best-fit Voigt peak positions of the spectra in Figure 4.3(d). In the medium-power regime, there is a continuous but quick peak shift of about $\sim 1 \mathrm{GHz}$ for trap configurations (11), (01), and (10). This is possibly caused by partial screening of the electric fields of the trapped charges by the free charge carriers introduced by the above-band excitation. Figure 4.5(b) shows the spectrally integrated RPLE of the QD as a function of above-band laser power. It reaches its maximum value at $1.2 \times 10^{-6} \mathrm{P}_{1}$, indicating that the local charge environment around the QD most favors neutral exciton emission (over trion or biexciton emission) at that power. When the above-band laser goes over this threshold, the QD fluorescence starts to decrease, reflecting the fact that more free charge carriers are available for the QD to capture in favor of trion and biexciton emission, which reduces neutral exciton emission. As the data shows, the QD resonant emission is suppressed by a factor of 2 in the ultrahigh-power regime compared to the maximum emission intensity at medium power. Figure 4.5(c) shows the Voigt line widths of each individual peak extracted from the fitting. The heavily overlapping of peaks introduces significant uncertainty to this parameter, which prohibit us from investigating the line-width evolution of single peak. However, the statistical average of the line widths at each above-band power still contains the information on broadening induced by the environmental fluctuation, since this fluctuation is coherent to all charge trap configurations. As depicted by the yellow shaded region in Figure 4.5(c), the mean line width drops $28 \%$ (from $1.25 \mathrm{GHz}$ to $0.8 \mathrm{GHz}$ ) as the above-band excitation power increases, implying that the presence of a proper level of free charge carriers around the QD is beneficial on suppressing the broadening caused by the environmental fluctuation.

All the RPLE peaks show a gradual spectral drift from the low-power regime to the ultrahigh-power regime. This is possibly caused by an asymmetric distribution of many distant charge traps that are far from the QD. By investigating the evolution of the green peaks [configuration (00)], we determined the drift to be an approximately $0.6 \mathrm{GHz}$ redshift. This redshift happens to follow the same trend of QD energy drift when the 
local temperature is increased by a small amount, about $0.2 \mathrm{~K}[168,169]$. However, other dots show a blue shift of similar amount over the same above-band excitation power range, as shown in Figure 4.4(b). Therefore thermal heating by the laser is not sufficient to explain these observations. In addition, a calculation with a simple two-dimensional thermal conductance model with the maximum above-band power used here (thermal conductivity of GaAs at $4 \mathrm{~K}$ is $10 \mathrm{Wcm}^{-1} \mathrm{~K}^{-1}$, and absorption coefficient at $632 \mathrm{~nm}$ is $4 \times 10^{4} \mathrm{~cm}^{-1}$ ) shows that the temperature rise would be less than $0.2 \mathrm{mK}$ (see Appendix A4.2 for calculation detail). This is too small to account for the observed spectral shift.

\subsubsection{Correlation function of resonance fluorescence}

The RPLE data quantify both the magnitude of the Stark shifts experienced by the QD and the time-averaged occupation probability of the charge traps that cause them. The time dependent behavior of the charge traps
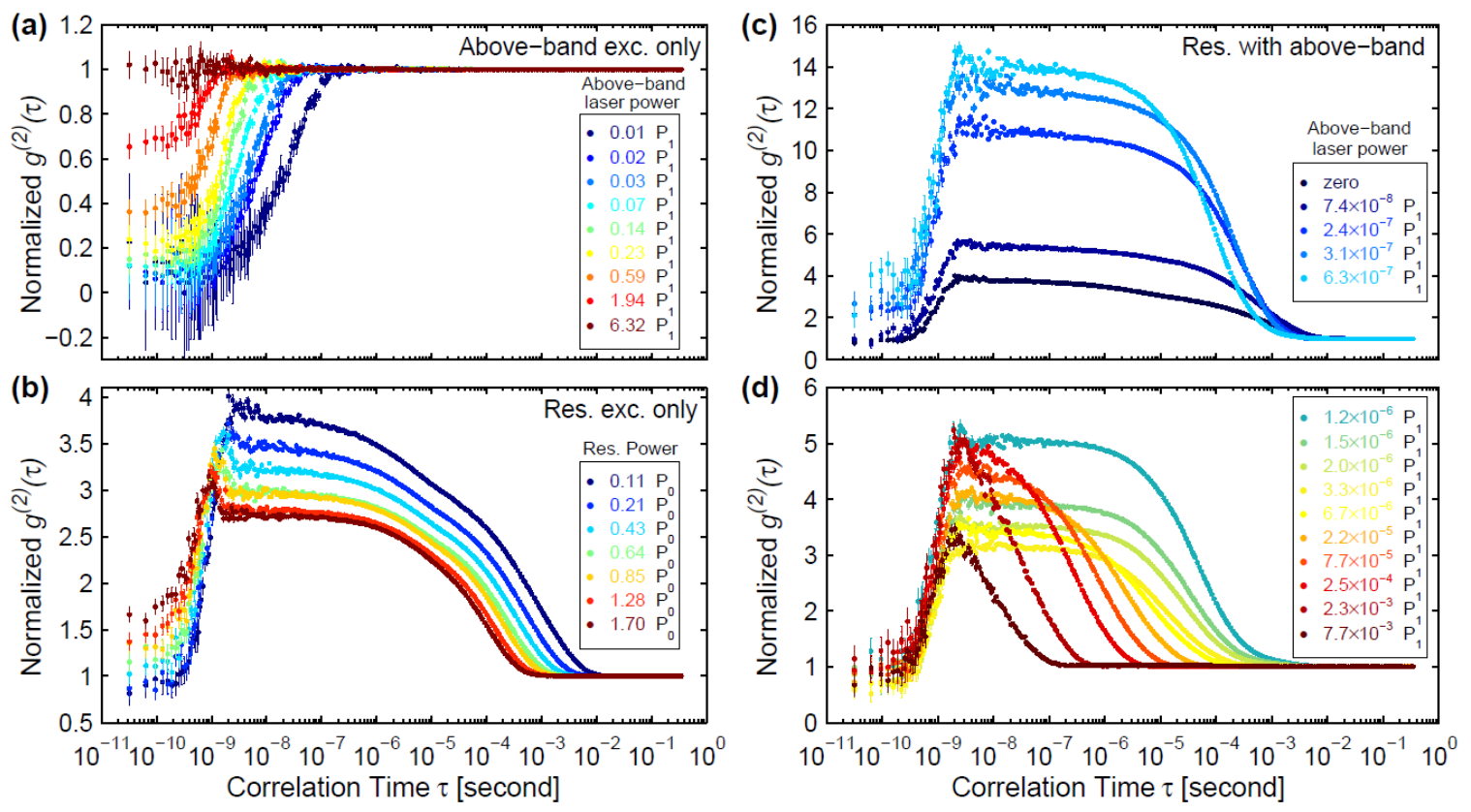

Figure 4.6 Second-order correlation function, $g^{(2)}(\tau)$, of the fluorescence from QD2 when the sample is illuminated with (a) only above-band laser, (b) only resonant laser, or (c-d) both lasers. The power of the lasers are listed in the legend in the fractions of the saturation power, $\mathrm{P}_{0}$ for resonant beam and $\mathrm{P}_{1}$ for above-band beam. (a) $g^{(2)}(\tau)$ under above-band excitation. The anti-bunching dip shows a power-dependent evolution. No bunching is present in this case. (b) $g^{(2)}(\tau)$ under resonant excitation. The resonant laser is tuned to $+1.91 \mathrm{GHz}$ from zero-detuning, the same as the black dot on zero-power line in Figure 4.3(d). (c) $g^{(2)}(\tau)$ measured at the black dots in box A in Figure 4.3(d). The resonant laser is tuned to $\sim 1.96 \mathrm{GHz}$ detuning at a power of $0.11 \mathrm{P}_{0}$ and the above-band laser power is varied from zero to $6.32 \times 10^{-7} \mathrm{P}_{1}$. A significant increase of bunching amplitude and small shrinking of bunching time can be seen. (d) $g^{(2)}(\tau)$ from box B in Figure 4.3(d). The resonant laser is at $\sim 0.56 \mathrm{GHz}$ detuning with a power of $0.11 \mathrm{P}_{0}$. The power of above-band laser is varied from $1.24 \times 10^{-6} \mathrm{P}_{1}$ to $7.69 \times 10^{-3} \mathrm{P}_{1}$. 
under $\mathrm{cw}$ excitation can be characterized by the photon statistics of the resonance fluorescence. Figure 4.6 shows the measured second-order correlation function, $g^{(2)}(\tau)$, of QD2 under three different excitation regimes: (a) with only above-band excitation, (b) with only resonant excitation, and (c-d) with resonant excitation accompanied by a small amount of above-band laser beam. All correlation data are normalized to the long-time value at 0.2 seconds and plotted on a logarithmic time scale so both the short-time and long-time behavior can be clearly seen. All data show a clear dip near $\tau=0$, a sign of antibunching expected of emission from a single quantum emitter. Although $g^{(2)}(0) \geq 1$ is present for most curves, all data, however, do show $g^{(2)}(0)<$ $g^{(2)}(\tau)$ for some values of $\tau$, which basically ensure QD2 as a non-classical photon emitter. In fact, the experimentally observed $g^{(2)}(0) \geq 1$ in Figure $4.6(\mathrm{~b}-\mathrm{d})$ is mostly caused by a convolution effect, where the sharp intrinsic antibunching dip is averaged out in convolution with the bunching plateau due to the finite response time of the detectors ( $\sim 0.8 \mathrm{~ns})$. This is the similar effect that is also responsible for smoothing out of the oscillations in Figure 3.12(a).

It is apparent from Figure 4.6 that the photon bunching only occurs when the resonant excitation is employed. But the single condition of resonant excitation cannot cause photon bunching. There must be some other physical processes that are capable of "regularly chopping" the QD emission involved to cause this phenomenon. In our system, two mechanisms are responsible for this intermittent disturbance of the resonant excitation of the QD: local electric environment fluctuation, and charging of the QD. When the resonant laser is tuned to one of the peaks in the RPLE spectrum, the QD will emit strong fluorescence. If a nearby charge trap switches from unoccupied to occupied, or vice versa, the QD resonance will shift and the fluorescence intensity will be reduced. This effect manifests as bunching in $g^{(2)}(\tau)$ on the time scale of the trap switching rate.

Secondly, in contrast with QDs in a diode structure [170-172], here the charge state of the QD in the sample is uncontrolled, and the QD may capture a charge from the environment. The resonant laser is tuned to the neutral exciton transition and when the QD is charged that transition is not available. Thus, when the QD is charged, there will be no resonance fluorescence. The charged QD may capture another charge of the opposite polarity from the environment, forming an exciton and returning to a neutral condition. Thus the QD may switch between charged and neutral states, emitting resonance fluorescence while neutral and no fluorescence while charged. Therefore the emitted photons will be bunched on a time scale similar to the time it takes the QD to capture a charge.

With solely above-band excitation, no photon bunching is observed for all excitation powers from $7.0 \times 10^{-3} \mathrm{P}_{1}$ to $6.3 \mathrm{P}_{1}$, and all the values of $g^{(2)}(0)$ are well below the classical limit of 1 (except for the highest power), as shown in Figure 4.6(a). As the excitation power increases, the depth of the anti-bunching dip starts to decrease, i.e., $g^{(2)}(0)$ starts to increase, caused by the stronger emissions from other photon sources at higher excitation power. This observation is evidenced by the raising of the RPLE background observed in the ultra-high power regime in Figure 4.3(d). Meanwhile, the width of the dip shows a compelling effect of broadening with the decreasing excitation power. The width even reaches $\sim 15 \mathrm{~ns}$ (full width at half maximum) at an excitation power of $0.01 \mathrm{P}_{1}$. This value is 29 -fold longer than the lifetime of QD2, implying that the dip width does not only determined by the recombination rate of the confined electron-hole pair, but also relies on the capturing rates of these charges from the environment. 
Under resonant only excitation (resonance with high-energy state), an apparent photon bunching presents in all $g^{(2)}(\tau)$ at different excitation powers, as shown in Figure 4.6(b). All bunching plateaus experience an exponential decay, a sign of bunching, at longer times down to an asymptotic value. In a linear-logarithmic plot, an exponential decay is characterized by a high plateau followed by a sharp decay at the characteristic time of the exponential, finishing with another lower plateau. The height of the plateau at intermediate $\tau$ values indicates the degree of bunching. Correlation functions like this indicate that on short-time scales the emitted photons are separated by the lifetime, but on long-time scales the photon stream is separated into bunches. All $g^{(2)}(\tau)$ data presented here show not a single exponential decay, but multiple exponential decays with different characteristic times $\left(10^{-7} \mathrm{~s} \sim 10^{-2} \mathrm{~s}\right)$, which is consistent with the earlier study [125]. Since no above-band laser involved, the QD excitation is disturbed by the intrinsic free charge carriers introduced by the intrinsic doping or defects in the sample.

As the power of resonant excitation increases, the peak of Rabi oscillation (see Figure 4.6(b)) shows a blue shift, moving towards shorter time scale, as expected. The same shift is also responsible for the rising of the antibunching dip, i.e., the increase of $g^{(2)}(0)$, because the instrument response function (IRF) of the detectors [see inset of Figure 2.6(d)] covers more plateau as it moves to shorter time scale. Since $g^{(2)}(\tau)$ is normalized, the bunching height or decay time should not be affected by the excitation power if it only modifies QD's emission rate. However, the decrease of the bunching height and speed-up of the decay time as increasing excitation power suggests that the excitation photons do have an impact on the QD's local environment. This conclusion is consistent with evolution of the spectrum amplitude observed from QD1, as shown in Figure 3.10(c). Based on the data, it is hard to pin down the exact mechanism from the possible schemes [60].

Figure 4.6(c-d) show how $g^{(2)}(\tau)$ is affected by the modification of the local free charge carrier density introduced by the above-band laser. To obtain the maximum signal-to-noise ratio in $g^{(2)}(\tau)$, we followed the brightest peak position in the RPLE map to collect the correlation data; the points of collection are marked by black dots in Figure 4.3(d). Figure 4.6(c) shows the $g^{(2)}(\tau)$ results measured at the points in box A and Figure 4.6(d) for those from box B. According to the fits to the RPLE data, when recording the correlations in Figure 4.6(c-d) the resonant laser is mostly exciting the high energy peak of the fine structure doublet for either trap configuration (00) for box A or trap configuration (11) for box B.

As the above-band laser power increases, the bunching amplitude in Figure 4.6(c) increases monotonically up to 14 due to the decrease of the probability of trap configuration (00) as indicated by the decreasing of fluorescence in Figure 4.3(d). Bunching means that the overall emission is grouped into clumps of photons, and there is a dearth of photons between the bunches. An increase of the bunching amplitude reflects a reduction in the relative probability of detecting two photons separated by a long time interval. This indicates that the QD is turned into an "off" state or low count-rate state more frequently. If the QD turns "off" more frequently, it reduces the number of photon pairs with a long separation interval compared to those with a short separation interval. This unbalanced change leads to an increase of the relative probability to find a photon pair with a short separation interval, i.e., the increase of the $g^{(2)}(\tau)$ bunching amplitude. Pronounced intensity fluctuation (high bunching level) for the medium-power regime in both Figure 4.6(c-d) is associated with accelerated transitions 
between the different charge trap configurations. This leads to strong intensity fluctuations of the PL that monitors one of the particular configurations, e.g., the (00) state for Figure 4.6(c) and the (11) state for Figure 4.6(d). This acceleration is reflected in the shortening of the characteristic decay time of the bunching in $g^{(2)}(\tau)$, from 10 to $1 \mathrm{~ms}$ for Figure 4.6(c) and from a few milliseconds to a few microseconds for Figure 4.6(d) up to the top of the high-power regime.

In contrast to the decay time, in this power range $\left(1.2 \times 10^{-6} \mathrm{P}_{1} \sim 2.5 \times 10^{-4} \mathrm{P}_{1}\right)$, the bunching amplitude shows a non-monotonic behavior. First, it decreases from 5 to 3 when the above-band power increases up to $3.3 \times 10^{-6} \mathrm{P}_{1}$, then it rises back up to 5 at $2.5 \times 10^{-4} \mathrm{P}_{1}$, and finally it decreases again. The first decrease is due to the increasing probability for the traps to be in configuration (11), which is the charge configuration with which the laser is in resonance. In fact, an increase in PL at the same above-band power in the RPLE spectra gives a direct support for this argument; see Figure 4.3(d). The following increase of bunching amplitude is associated with decrease of time-averaged total PL [Figure 4.5(b)] in the same power range: the QD starts to capture another charge, and the neutral exciton emission becomes less favored, while trion or biexciton population get correspondingly increased.

Due to the energy mismatch of the fluorescence from a trion (or a biexciton) to the Fabry-Perot mode of the cavity, these photons experience a low efficiency for coupling out of the DBR cavity. The light coupled out of the cavity are also filtered out by the monochromator. Therefore, these evolution paths serves as dark path to our observation and do not contribute to the measured correlation. And one would expect a greater bunching amplitude for a higher above-band power. However, the opposite trend is observed in Figure 4.6(d) when the above-band power is more than $2.5 \times 10^{-4} \mathrm{P}_{1}$. It seems that the antibunching dip prevents the bunching amplitude from increasing further at short time scales. Physically, this is because there are rarely photon pairs with time interval shorter than the lifetime of the QD. If the lifetime of the QD was shorter, one would expect the bunching amplitude to continue rising. In addition, the high density of free charge carriers in the ultrahighpower regime enables both the QD and other sources (e.g., the continuum tail of wetting layer defect states) to emit photons without resonant excitation. These extra photons would fill the gaps between the bunching of the resonant-excited-QD emitted photons, leading to a slight decrease of the antibunching dip depth and a decrease of the bunching amplitude. It is possible that the decrease of the dip depth in Figure 4.6(c) is mostly due to the finite response time of the detectors rather than simultaneous photon emissions from multiple sources, based on the fact that a larger $g^{(2)}(0)$ value is accompanied by the higher bunching plateau. Thus, up to the above-band power of $10^{-6} \mathrm{P}_{1}$, the resonantly excited QD along with its environment as a whole would still be a good single photon source.

We note that several essential studies on closely related topics were published in the past few years, such as quantum dot charging [61] and nearby charge trap dynamics [60]. A brief summary of those works and a comparison to our study are provided here. The QD studied by Nguyen et al. [61] has an above-band excitation saturation power of $30 \mu \mathrm{W}$, which is consistent with our value of $28.5 \mu \mathrm{W}$. They also note that their QD emits no resonance fluorescence without a particular very small amount of above-band laser power. This is similar in kind if not degree to our observation that the resonance fluorescence is a maximum with a nonzero above-band laser power. Without above-band excitation, Ngugen et al. extract a charge trap ionization and neutralization rate 
on the order of $10^{4} \mathrm{~s}^{-1}$, while our results show a large range of bunching decay rates from $10^{3}$ to $10^{5} \mathrm{~s}^{-1}$. This difference is probably due to the fact that our QD can emit resonance fluorescence without above-band excitation, leading to a $g^{(2)}(\tau)$ measuring both effects of QD ionization and charge trap dynamics. Nevertheless, this rate range is consistent with the study by Arnold et al. [60], where the trap loading/unloading rate varies from $4 \times 10^{3}$ to $6 \times 10^{4} \mathrm{~s}^{-1}$, although their QD shows much larger discrete Stark shift ( $\left.18 \mathrm{GHz}\right)$ indicating either a much larger QD dipole moment or a much closer charge trap. Nguyen et al. also extracted a QD charging rate of $\sim 10^{4} \mathrm{~s}^{-1}$ at low above-band power $(0.01 \mathrm{nW})$, and of $10^{7} \mathrm{~s}^{-1}$ at high above-band power $(230 \mathrm{nW})$. Correspondingly, at the same above-band power, our transition rate is $\sim 10^{4} \mathrm{~s}^{-1}$ at $6.3 \times 10^{-7} \mathrm{P}_{1}$ and $~$ $10^{8} \mathrm{~s}^{-1}$ at $7.7 \times 10^{-3} \mathrm{P}_{1}$. This is a difference of one order of magnitude at high above-band power, which can be understood by noticing that our QD experiences two sources of fluctuation, charging of QD itself and ionization of nearby charge traps, while Nguyen's QD only experiences the former one. Thus the fluctuation of nearby charge traps increases the bunching decay rate. From Arnold's study, the transition rate of the charge trap is found to be $\sim 1.6 \times 10^{6} \mathrm{~s}^{-1}$ for resonant excitation at $230 \mathrm{nW}$. This value is too low to explain the rate difference between our study and Nguyen's study at high above-band power. But it is possible that this number would be significantly higher when using above-band excitation rather than below band-gap resonant excitation, and thus bridging the difference. Qualitatively, our $g^{(2)}(\tau)$ bunching decay rate shows a linear relationship with the above-band excitation power, which is consistent with the results of both Nguyen et al. and Arnold et al. There, the extracted transition rate is proportional to either the above-band power or the square root of the power, but at the powers of interest here the larger linear term dominates if all effects are present in the data. Two other works observing discrete Stark shifts are Houel et al. [54] using differential reflection spectroscopy and Hauck et al. [173] using differential transmission spectroscopy. Both studied a sample with a Schottky diode structure that is different from ours, but the values of the discrete shifts are close to those presented here. Moreover, their interpretation of the phenomenon involves charges trapped around the QD with a distance of 100 nm [54] or $\sim 30 \mathrm{~nm}$ [173], which is close to the result of our calculation shown in the next section. 


\subsection{Possible Trap Locations}

Knowing the Stark shifts experienced by the QD allows us to calculate the possible positions of the charge traps. By fitting the data in Figure 4.3(a), we obtain four different values of the Stark shift, which we attribute to the four possible charge configurations of two nearby charge traps. We assume that the charge configuration that is most likely with zero above-band laser power corresponds to the equilibrium configuration, (00), where both charge traps are neutral. We define the corresponding Stark shift to be zero: $\Delta v_{0}=0 \mathrm{GHz}$. The other three Stark shifts are

$$
\begin{aligned}
& \Delta v_{1}=-3.3607 \mathrm{GHz}(+0.0201 /-0.0064 \mathrm{GHz}), \\
& \Delta v_{2}=+1.1189 \mathrm{GHz}(+0.1064 /-0.0028 \mathrm{GHz}), \\
& \Delta v_{3}=-2.2145 \mathrm{GHz}(+0.1054 /-0.0053 \mathrm{GHz}),
\end{aligned}
$$

where $\Delta v_{1}$ corresponds to configuration (10), $\Delta v_{2}$ corresponds to configuration (01), and $\Delta v_{3}$ corresponds to configuration (11). We make the assignment of $\Delta v_{3}$ to the doubly charged configuration because it corresponds to the predominant fluorescence peak at high above-band laser power. Both traps being charged is the most likely configuration when the above-band laser is producing many free charges that may be captured by the traps.

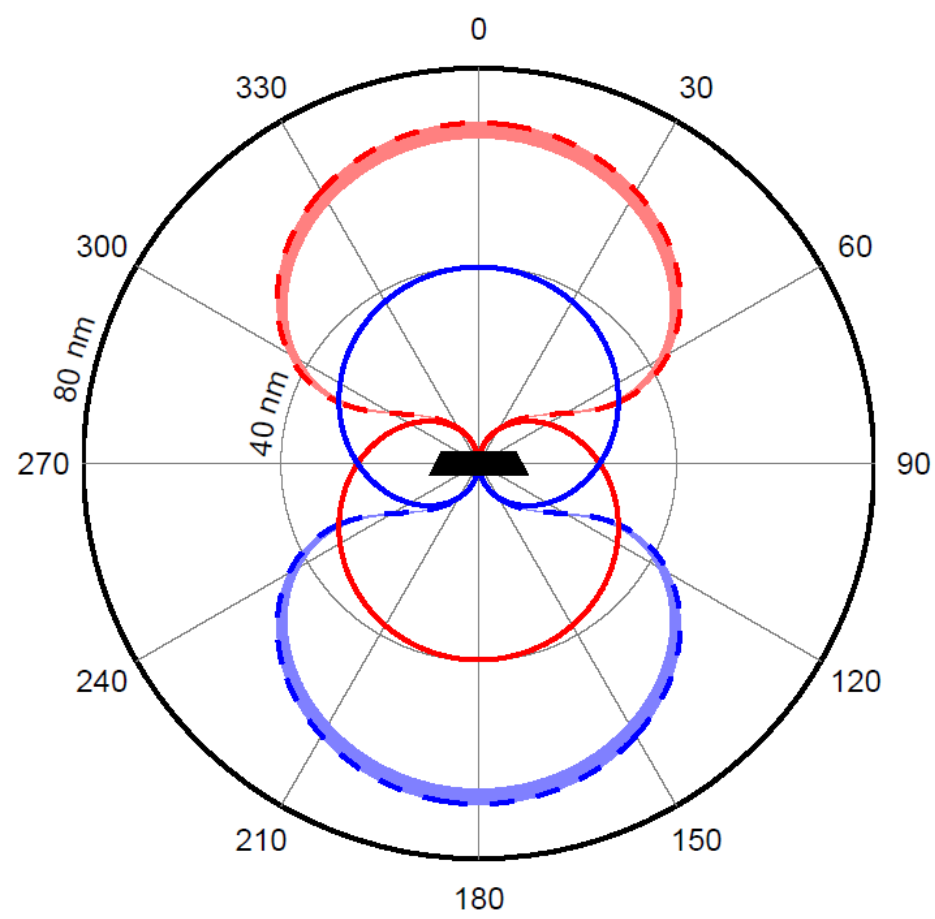

Figure 4.7 Possible charge trap locations consistent with the measured Stark shifts. Red and blue correspond to positive and negative trap polarity, respectively; the QD is represented schematically at the origin. The solid lines are for trap $\alpha$; the dashed lines are for trap $\beta$. The shaded regions denote locations consistent with the confidence range of $\Delta v_{2}$; the confidence range of $\Delta v_{1}$ is small enough that the corresponding region is hidden by the solid lines. 
We can combine Eqns. (4.2) and (4.3) to determine the possible positions $\boldsymbol{r}_{\boldsymbol{i}}$ that are consistent with the known values $\Delta v_{i}$ of the resonance shift. For a single charge trap, this results in an equation that relates the distance $r_{i}$ between the QD and the trap to the polar angle $\theta_{i}$ between the $\hat{\mathrm{z}}$ axis and the vector $\boldsymbol{r}_{\boldsymbol{i}}$ :

$$
\Delta v_{i}=\frac{p k q_{i}}{h r_{i}^{2}} \cos \left(\theta_{i}\right)-\frac{\beta k^{2} e^{2}}{h r_{i}^{4}} \sin ^{2}\left(\theta_{i}\right)
$$

where $k \equiv 1 /\left(4 \pi \epsilon_{0} \epsilon_{r}\right)$ and $q_{i}$ is the charge of the trap when ionized. Since the RPLE data cannot distinguish the polarity of the traps when they are charged, we do not know the sign of $q_{i}$. Thus we consider both the case where the charged trap is positive $\left(q_{i}>0\right)$ and the case where it is negative $\left(q_{i}<0\right)$. Here, we use typical values for the dipole moment [173] and polarizability [174] of self-assembled InGaAs QDs: $\boldsymbol{p}=e \times(0.2 \mathrm{~nm}) \hat{\mathrm{z}}$ and $\beta_{x x}=\beta_{y y}=\beta=4 \mu \mathrm{eV} /(\mathrm{kV} / \mathrm{cm})^{2}$, and $\epsilon_{r}=13.1$ for GaAs. Using the values and confidence intervals of $\Delta v_{1}$ and $\Delta v_{2}$ and Eqn. (4.7), we can determine the sets of possible values $\left(\mathrm{r}_{1}, \theta_{1}\right)$ and $\left(\mathrm{r}_{2}, \theta_{2}\right)$. Each set of possible positions defines an azimuthally symmetric volume in the space around the QD. Figure 4.7 shows colored areas which are cross-sections through these volumes for both possible polarities (red = positive; blue = negative) of trap $\alpha$ (solid lines) and trap $\beta$ (dashed lines); a schematic of a typical 20-nm diameter QD is shown at the origin.

From Figure 4.7, we can see that to cause the measured Stark shifts, a charge trap must be less than $70 \mathrm{~nm}$ away from the QD, which is relatively close: less than four QD diameters. Thus any charge trap located at the DBR interfaces or surface is too far away to cause these discrete spectral shifts. Two charge traps within a spherical volume of radius $70 \mathrm{~nm}$ is consistent with the typical unintentional doping concentration of $10^{15} \mathrm{~cm}^{-3}$ for GaAs grown by molecular beam epitaxy. Notice that trap $\beta$ must be either above or below the plane of the QD, depending on its polarity, while trap $\alpha$ could be above or below the QD plane regardless of its polarity. The separation between the resonantly excited QD and trap $\alpha$ is $30.6 \mathrm{~nm}$ if the trap is in the wetting layer (see Figure 4.7). For comparison, in a sample with a high-density of self-assembled QDs [175] $\left(\sim 9.5 \times 10^{9} \mathrm{~cm}^{-2}\right)$, the average dot-to-dot distance is about $103 \mathrm{~nm}$, which is not much larger than the separation between the QD and trap $\alpha$ if it is in the wetting layer plane. Thus it is possible that trap $\alpha$ is another QD; however, this neighboring QD would be constrained to have only two charge states to be consistent with the RPLE data. Regardless of the identity of trap $\alpha$, trap $\beta$ cannot be another QD. 


\subsection{Conclusion}

In this study, resonant excitation spectroscopy successfully characterizes the local electric environment of the QD by providing detailed information about the number of nearby charge traps, their distances from the QD and their time-averaged occupation probability. Combined with weak above band-gap excitation below the level required to produce photoluminescence, the evolution of the local environment with respect to different densities of free charge carriers was studied, and we found that to achieve the maximum of total PL from the QD, a small amount of above-band excitation is required [Figure 4.5(b)]. This is similar to previous work on resonantly excited QDs [60-62,137,176], but here the behavior is more complicated. For the QD used in this study, the data indicate that there are two nearby charge traps within $70 \mathrm{~nm}$. Their exact locations depend on the polarity of the trap when ionized, which the current measurement techniques are unable to determine.

Correlation measurements give information about the time scale of switching from neutral to charged for both the traps and the QD. As the above-band excitation laser increases the density of free charge carriers, the time scale of the charge trap dynamics speeds up, decreasing the switching time, which is reflected in the decay time scale of the correlation bunching amplitude of resonantly excited fluorescence. This time scale spans five orders of magnitude from $10^{-2}$ to $10^{-8} \mathrm{~s}$. Given the very long time scales of bunching with zero or low above-band laser power, it is possible that many previous experiments did not recognize that the resonance fluorescence was bunched. We also note that the fastest bunching decay time of $10^{-8} \mathrm{~s}$ is only one order of magnitude longer than the anti-bunching time of about $10^{-9} \mathrm{~s}$. In that case, the equivalent photon stream would be bunches of fewer than 10 photons, and long stretches of time with no emission between the bunches.

The combined techniques of resonant excitation spectroscopy and resonant fluorescence correlation can determine many details of the local charge environment of a single QD. The QD chosen for this work exhibits multiple spectral behaviors: discrete spectral jumps, continuous spectral shift, and spectral diffusion. These behaviors have been observed before by others, but the techniques demonstrated here allow quantitative investigation of the details. No electrical contacts are necessary as the measurement is entirely optical. A QD that may be a suitable source of indistinguishable photons can be investigated using the same techniques to determine its potential. 


\section{Chapter 5: Outlook}

Photons generated by self-assembled quantum dots are gifted with many virtues, such as high single-photon purity, narrow spectral line-width and a good spin-photon interface, and they promise to be ideal flying qubits to convey information between individual quantum nodes [177]. However, intermittent fluorescence (or blinking) exhibited by these quantum emitters and spectral diffusion introduced by semiconductor environment limit the potential for practical use. Meanwhile, the physical process of relaxation of a free charge carrier into a quantum dot is still an open question, and the role played by the wetting layer in relaxation is not clear. Understanding the underlying mechanism is critical for suppressing the charging dynamics of quantum emitter, and it is also beneficial for improving quantum efficiency of light emitting applications. Chapter 4 of this dissertation has investigated these dynamics by varying free charge carrier density with different levels of above band-gap excitation powers. The second-order correlation measurements $\left(\right.$ see $g^{(2)}(\tau)$ in Figure 4.6) under different excitation conditions capture the charge dynamics of the QD including relaxation process, while it also measures the charge dynamics of nearby charge traps via spectral diffusion. These two phenomena intertwine with each other, and significantly complicate the physical model. For example, Figure 5.1 shows a rate model that considers the charge dynamics of the dot and the two nearby charge traps, the case of QD2 studied in Chapter 4. This is a simplified model since it does not include the charge carrier transfer from the environment to the dot or the traps. Nevertheless, it already contains a large number of fitting parameters that diminishes the possibility to pinpoint the best fit out of the tremendous parameter space considering normal computation power. Alternatively, direct measurement of part of the dynamics can significantly shrink the parameter space for later analysis. One way to do this is to modulate the above-band laser light with an AOM, as shown in Figure 5.2(a). By histogramming each time-tagged photon event with respect to the corresponding modulation signal, the effect of introducing free charge carriers into the system is imprinted in the time-evolution of the QD's fluorescence, as shown in Figure 5.2(b). A trion is constantly excited by the resonant laser but only when the above-band laser is on; the fluorescence starts as indicated by the rising edge of the PL signal. When the above-band laser is off, the PL suddenly drops to zero, reflecting the influence of the resonant laser on the charge state of the QD. The rising or decaying edge of the PL signal thus directly provides us the time scale of the dynamics after an exponential fitting.

In the above experiment, a HeNe laser is used to perturb the QD environment. This above band-gap light can directly promote the valence-band electrons in GaAs spacer layer to conduction band, creating a free charge carrier reservoir. These free charge carriers can follow different paths to relax into QDs, for example, via chargephonon interaction, or Auger process, or relaxing into the wetting layer before captured by QDs. The simultaneous presence of multiple dynamics in an experiment makes it difficult to interpret the data, especially for the case when two dynamics share a similar rate. However, if one can use a longer wavelength to excite the sample, for example, the light resonant with wetting layer but not energetic enough to cross GaAs band-gap. This would turn off the dominant source of free charge carriers in GaAs spacer, leaving the possibility to investigate the charge dynamics between the wetting layer and QDs. Furthermore, the resonant power-dependence of the spectrum amplitude shown in Figure 3.10(c) for QD1 and Figure 4.5(b) for QD2 implies that the resonant laser does not only excite the QD, but also affects the period of the time that the QD is available to be resonantly 
(a)
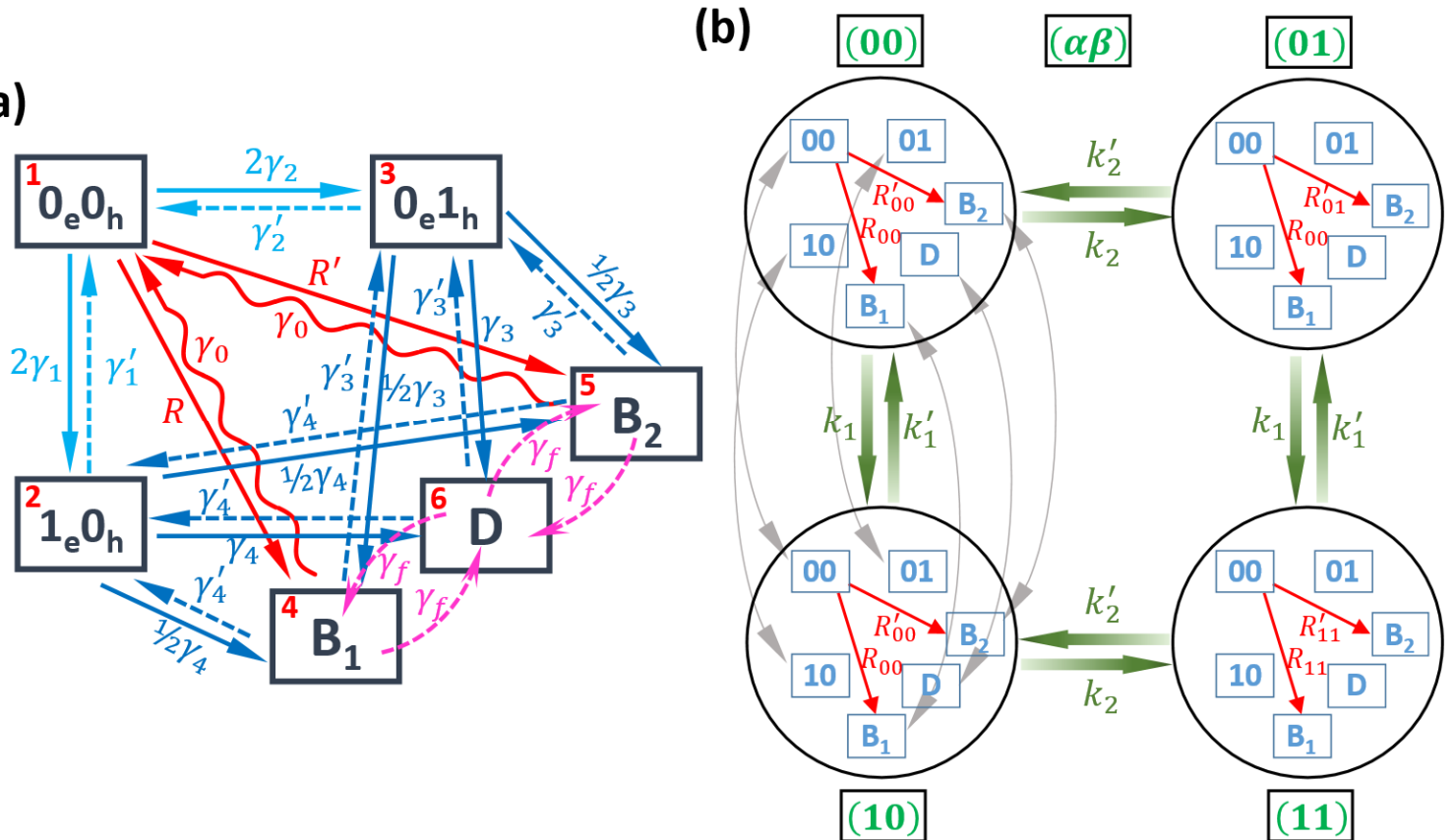

Figure 5.1 Model of the charge dynamics for QD2 and its two nearby charge traps (studied in Chapter 4). (a) Model of the charge dynamics of QD2. Each charge state is labeled by the number of confined electrons and holes in the black square boxes, where $\mathrm{B}_{1}, \mathrm{~B}_{2}$ represent the two bright exciton states, respectively, with fine structure splitting, and D represents the two dark exciton states. The processes of capturing charges from the local environment are indicated by solid arrows (with rates $\gamma_{1}, \gamma_{2}, \gamma_{3}$, and $\gamma_{4}$ ). The processes of losing charge carriers are indicated with dashed arrows $\left(\gamma_{1}^{\prime}, \gamma_{2}^{\prime}, \gamma_{3}^{\prime}, \gamma_{4}^{\prime}\right)$. The resonant excitation is represented by red straight arrows, and the radiative decay in red wavy arrows. The electron spin of an exciton can experience a flip by contact hyperfine interactions with nuclear spins at a rate of $\gamma_{f}$ or $\gamma_{f}^{\prime}$, as indicated by pink dashed arrows. Here, we only consider up to exciton states, because no trion or bi-exciton emissions are observed in QD's emission. (b) Model of the charge dynamics for two nearby charge traps (trap $\alpha$ and $\beta$ ). Two charge traps lead to 4 configurations, labeled by the charge state of trap $\alpha$ and trap $\beta$ as $(\alpha \beta)$ in the square boxes. Each black circle contains the corresponding QD states as shown in (a). The effect of different trap configuration ( $\alpha \beta)$ on the QD is reflected by different excitation rates $R_{\alpha \beta}$ and $R_{\alpha \beta}^{\prime}$, due to the quantum confined Stark effect (see Chapter 4). Since each charge trap and the QD are independent, any QD state can switch from one trap configuration to the other when charge trap configuration changes, as indicated by the double-sided grey arrows. For clarity, only one switching process is shown in the figure, i.e., between trap configuration (00) and (10) with a rate of $k_{1}$ or $k_{1}^{\prime}$ depending on the switching direction. This leads to a resultant 24 differential equations with $24 \mathrm{QD}$ states.

excited. This phenomenon can be investigated by doing a similar experiment as shown in Figure 5.2(a), but with $\mathrm{HeNe}$ replaced by a laser that is slightly red-detuned from resonance.

The charge dynamics of nearby or far-away charge traps, activated by the free charge carriers, can alter the color of the photons scattered by the QD over time, and thus impairs the indistinguishability of the scattered photons. This is troublesome for quantum applications that rely on two-photon interference, or the Hong-Ou-Mandel 

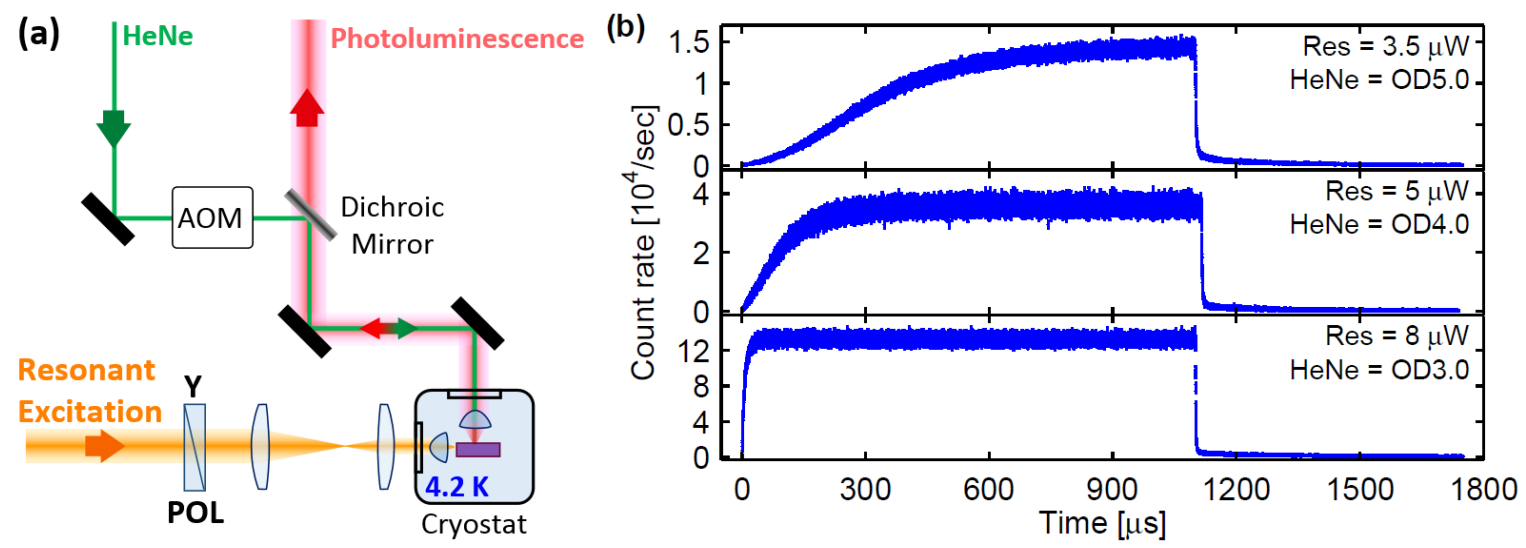

Figure 5.2 (a) Optical setup for AOM modulated experiment. The AOM has been inserted into the above-band laser path to modulate the power of the HeNe. Except for this part, all other optical paths are identical to Figure 2.6. (b) Time evolution of the QD's PL at different resonant excitation powers and above-band laser powers. The fluorescence is from the trion transition of a charged QD. The powers of the two lasers are indicated in each subplot. The rising edge and decaying edge of the signal correspond to the turn-on and turn-off event of the HeNe beam controlled by the AOM.

effect [56], where a pair of indistinguishable photons incident on a 50:50 beam splitter from two separate entrances will always leave the beam splitter from the same exit. The performance of this interference highly depends on the indistinguishability of two photons that is mainly characterized by the color, the polarization, the spectral and spatial envelope of each individual photon. Under the influence of the electric fluctuations shown in Chapter 4, caused by the charge dynamics of two nearby charge traps located within a $70 \mathrm{~nm}$ range from the QD, the resultant spectral diffusion is significant, and the scattered photons can be completely distinguishable from each other as indicated by the discrete spectral lines in Figure 4.3. One way to overcome this problem is to use coherent scattering. Because the coherent scattering is always at laser wavelength, it is inherently immune to the environmental fluctuation. Early works $[18,72,74]$ have demonstrated that coherently scattered photons exhibit a sub-natural line width that is about 2 orders of magnitude narrower than the lifetime limited line width. The slight shift of the QD's energy levels has little effect on coherent scattering. It has been demonstrated that these coherently scattered photons not only preserve the single photon emission properties [18], but also inherit the phase of the excitation laser [74]. The latter property enables a new method of photonic state engineering of single photons via manipulating the waveform of the excitation field. However, high-purity coherent scattering can only be obtained at low excitation power for a single 2-level system, namely the "Heitler regime" $[18,73]$. In Chapter 3 of this dissertation, we proposed that a 3-level system composed of a pair of orthogonal dipole moments is a better source of coherent single photons compared to a single 2-level system, with respect to both coherent scattering intensity and percentage as indicated by Figure 3.4(c). The 3-level system is capable of generating a brighter stream of single photons with sub-natural line width thanks to the interference between the coherent scattering from the two dipoles. The same interference is also responsible for the unconventional excitation line shape exhibited by the neutral QD. Because the 3-level system consists of two transitions, one expect that the coherent scattering bandwidth is probably broader than that of a single 2-level system, where the bandwidth is approximately the line width of the single transition. This means that 3-level system probably can generate shorter single photon pulse or single photon wave-packet with more complicated envelope function. 


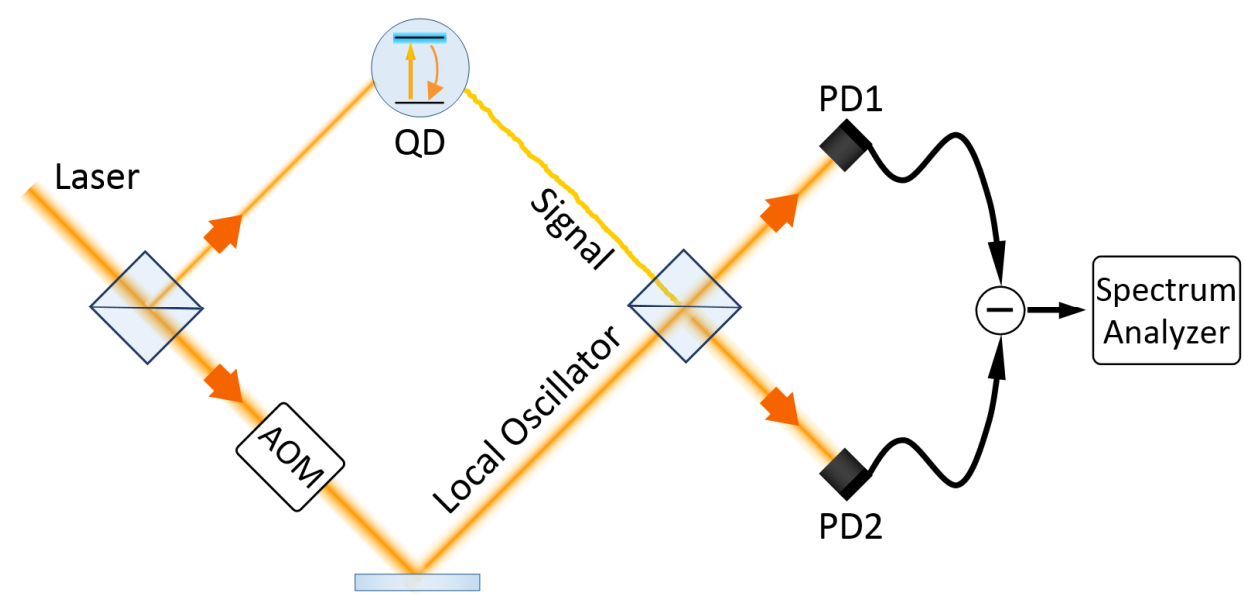

Figure 5.3 The heterodyne measurement setup. The setup is similar to the one used in reference [74]. The resonant excitation laser is split into two parts, where the small fraction excites the QD, while the other is frequency-shifted by an acousto-optic modulator (AOM). The QD scattered light is then combined with the strong LO beam at second beam splitter. The two outputs are recorded by two balanced photodiodes. The readings are first subtracted from each other to remove the DC component of the signal before being spectrum analyzed via Fourier transformation, where the coherent scattering spectrum can be extracted from the timedependent intensity.

The above arguments are worth some experimental efforts for a verification. Experimentally, the coherent scattering can be measured by using a heterodyne setup as shown in Figure 5.3. A fraction of the excitation beam is split off by a beam splitter to excite the QD, while the transmission portion serves as a local oscillator (LO) to interfere with the fluorescence from the QD. To enable lock-in detection of the weak QD signal, the frequency of the local oscillator is slightly shifted off by $\Delta v$ by using an acousto-optic modulator (AOM). The final optical intensity detected by the two balanced photodiodes (PD1 or PD2) has the mathematical form

$$
I_{i} \propto\left|E_{\mathrm{CS}}+E_{\mathrm{IS}}+E_{\mathrm{LO}}\right|^{2}=\left|E_{\mathrm{CS}}\right|^{2}+\left|E_{\mathrm{IS}}\right|^{2}+\left|E_{\mathrm{LO}}\right|^{2}+E_{\mathrm{CS}} E_{\mathrm{LO}} \cos \left(\Delta v \cdot t+\varphi_{i}(t)\right)
$$

where $E_{\mathrm{CS}}, E_{\mathrm{IS}}$ are the coherently and incoherently scattered fields from the $\mathrm{QD}, E_{\mathrm{LO}}$ is the local oscillator field, and $\varphi_{i}$ is the time-dependent relative phase between the coherent scattering and LO field measured at detector $i$. The other two cross terms, $E_{\mathrm{IS}} E_{\mathrm{LO}}$ and $E_{\mathrm{CS}} E_{\mathrm{IS}}$, are averaged out to be zero due to the fast random variation of the phase carried by the incoherent scattered field $E_{\mathrm{IS}}$. The DC component of the detected signal (first three terms in Eqn. (5.1)) is then removed by subtracting the readings of the two PDs from each other. The residual sinusoidal signal corresponds to the last term in Eqn. (5.1), where the square of this oscillation amplitude gives the total coherent scattering intensity, and the Fourier analysis of the signal gives the coherent scattering power spectrum.

Since each transition of a neutral QD is equivalent to a 2-level system (at least for $\Omega_{\mathrm{R}}<10 \Gamma_{\mathrm{sp}}$ ), the coherent scattering from a 2-level system can be measured with the same QD but with the detection polarization aligned to one dipole moment. This avoids possible different environmental effects for different QDs and minimizes the discrepancy between different excitation wavelengths. To verify the indistinguishability enhancement, MachZehnder interferometry and Hong-Ou-Mandel experiment can be implemented. If a pair of fast avalanche 
photodiodes (bandwidth $>4 \mathrm{GHz}$ ) are available, the fast oscillation present in $g^{(2)}(\tau)$ (Figure 3.12(a) in Chapter 3.3.2) can also be verified. 


\section{Chapter 6: Conclusion}

Generation of indistinguishable single-photons is challenging for a solid-state system since it is difficult to avoid the fluctuations in the electric environment caused by inevitable imperfections implanted in the sample or device during growth or fabrication. This dissertation investigates these fluctuations by studying the evolution of the spectra of a QD while varying the local density of free charge carriers. We found that the charge dynamics of nearby or far-away charge traps are the culprit for the observed discrete jumps or continuous shift of the QD's spectra. Although the spectral wandering is not acceptable for generating indistinguishable photons, it does provide information for pinpointing the location of the nearby charge traps as demonstrated in the dissertation. By examining the photon statistics of the QD's emission, the time scale of these charge dynamics can be determined. In fact, the temporal correlation measurement captures both the non-classical nature of these quantum emitters and the charge dynamics of both the QD and nearby defects. These time-resolved measurements provide a new tool for studying the physical process of relaxation of a free charge carrier into a quantum dot, and unraveling the role played by the wetting layer in these relaxations. The understanding of this physical process is critical for suppressing the charging dynamics of quantum emitter, and it is beneficial for improving quantum efficiency of light emitting applications.

Considering the difficulty of obtaining ideally perfect samples with no defects, we proposed another way to overcome this problem by taking advantage of the coherent scattering from a 3-level V-shaped system. The coherent scattering bears several advantages compared to incoherent scattering: long coherence time, and the inheritance of the phase from the excitation laser. The latter property is useful for pulse-shape engineering of coherently scattered single-photons. For a 3-level system, it usually consists of two non-degenerate transitions whose wider spectral envelope is beneficial for scattering photons with arbitrary wave-forms compared to the single transition of a 2-level system. Moreover, we propose that a 3-level system, composed of a pair of orthogonal dipole moments, is a better system to generate coherently scattered single-photons than a single 2level system, in terms of both coherent scattering intensity and purity. Specifically, when both systems output the same coherent scattering intensity as the maximum value output by a single 2-level system, the coherent scattering purity is $89 \%$ (3-level) and $51 \%$ (2-level) respectively. Due to the presence of two scattering sources

- the two non-degenerate dipole moments - an interference effect is expected between the two coherent contributions. Experimentally, an unconventional excitation line-shape has been observed. The consistency between the theory and experiment substantiate our proposal on coherent scattering performance of a 3-level system. 


\section{Bibliography}

1. P. Michler, A. Kiraz, C. Becher, W. V. Schoenfeld, P. M. Petroff, L. Zhang, E. Hu, and A. Imamoglu, "A Quantum Dot Single-Photon Turnstile Device," Science 290, 2282-2285 (2000).

2. C. Santori, M. Pelton, G. Solomon, Y. Dale, and Y. Yamamoto, "Triggered Single Photons from a Quantum Dot," Phys. Rev. Lett. 86, 1502-1505 (2001).

3. N. Akopian, N. H. Lindner, E. Poem, Y. Berlatzky, J. Avron, D. Gershoni, B. D. Gerardot, and P. M. Petroff, "Entangled Photon Pairs from Semiconductor Quantum Dots," Phys. Rev. Lett. 96, 130501 (2006).

4. R. Trotta, J. S. Wildmann, E. Zallo, O. G. Schmidt, and A. Rastelli, "Highly Entangled Photons from Hybrid Piezoelectric-Semiconductor Quantum Dot Devices," Nano Lett. 14, 3439-3444 (2014).

5. A. Kiraz, C. Reese, B. Gayral, L. Zhang, W. V. Schoenfeld, B. D. Gerardot, P. M. Petroff, E. L. Hu, and A. Imamoglu, "Cavity-quantum electrodynamics with quantum dots," J. Opt. B Quantum Semiclassical Opt. 5, 129 (2003).

6. A. Badolato, K. Hennessy, M. Atatüre, J. Dreiser, E. Hu, P. M. Petroff, and A. Imamoğlu, "Deterministic Coupling of Single Quantum Dots to Single Nanocavity Modes," Science 308, 1158-1161 (2005).

7. K. Hennessy, A. Badolato, M. Winger, D. Gerace, M. Atatüre, S. Gulde, S. Fält, E. L. Hu, and A. Imamoğlu, "Quantum nature of a strongly coupled single quantum dot-cavity system," Nature 445, 896-899 (2007).

8. D. Englund, A. Faraon, I. Fushman, N. Stoltz, P. Petroff, and J. Vučković, "Controlling cavity reflectivity with a single quantum dot," Nature 450, 857-861 (2007).

9. P. Yao, V. s. c. Manga Rao, and S. Hughes, "On-chip single photon sources using planar photonic crystals and single quantum dots," Laser Photonics Rev. 4, 499-516 (2010).

10. J. Vuckovic, "Quantum optics and cavity QED with quantum dots in photonic crystals," ArXiv14022541 Cond-Mat Physicsphysics Physicsquant-Ph (2014).

11. S. Sun and E. Waks, "Single-shot optical readout of a quantum bit using cavity quantum electrodynamics," Phys. Rev. A 94, 012307 (2016).

12. C. Santori, D. Fattal, J. Vučković, G. S. Solomon, and Y. Yamamoto, "Indistinguishable photons from a single-photon device," Nature 419, 594-597 (2002).

13. O. Gazzano, S. Michaelis de Vasconcellos, C. Arnold, A. Nowak, E. Galopin, I. Sagnes, L. Lanco, A. Lemaître, and P. Senellart, "Bright solid-state sources of indistinguishable single photons," Nat. Commun. 4, 1425 (2013).

14. Y.-M. He, Y. He, Y.-J. Wei, D. Wu, M. Atatüre, C. Schneider, S. Höfling, M. Kamp, C.-Y. Lu, and J.-W. Pan, "Ondemand semiconductor single-photon source with near-unity indistinguishability," Nat. Nanotechnol. 8, 213-217 (2013).

15. E. B. Flagg, A. Muller, S. V. Polyakov, A. Ling, A. Migdall, and G. S. Solomon, "Interference of Single Photons from Two Separate Semiconductor Quantum Dots," Phys. Rev. Lett. 104, 137401 (2010).

16. E. B. Flagg, S. V. Polyakov, T. Thomay, and G. S. Solomon, "Dynamics of Nonclassical Light from a Single Solid-State Quantum Emitter," Phys. Rev. Lett. 109, 163601 (2012).

17. P. Gold, A. Thoma, S. Maier, S. Reitzenstein, C. Schneider, S. Höfling, and M. Kamp, "Two-photon interference from remote quantum dots with inhomogeneously broadened linewidths," Phys. Rev. B 89, 
035313 (2014).

18. C. Matthiesen, A. N. Vamivakas, and M. Atatüre, "Subnatural Linewidth Single Photons from a Quantum Dot," Phys. Rev. Lett. 108, (2012).

19. J. P. Reithmaier, G. Sęk, A. Löffler, C. Hofmann, S. Kuhn, S. Reitzenstein, L. V. Keldysh, V. D. Kulakovskii, T. L. Reinecke, and A. Forchel, "Strong coupling in a single quantum dot-semiconductor microcavity system," Nature 432, 197-200 (2004).

20. A. Bechtold, D. Rauch, F. Li, T. Simmet, P.-L. Ardelt, A. Regler, K. Müller, N. A. Sinitsyn, and J. J. Finley, "Three-stage decoherence dynamics of an electron spin qubit in an optically active quantum dot," Nat. Phys. 11, 1005-1008 (2015).

21. G. Wüst, M. Munsch, F. Maier, A. V. Kuhlmann, A. Ludwig, A. D. Wieck, D. Loss, M. Poggio, and R. J. Warburton, "Role of the electron spin in determining the coherence of the nuclear spins in a quantum dot," Nat. Nanotechnol. advance online publication, (2016).

22. E. A. Stinaff, M. Scheibner, A. S. Bracker, I. V. Ponomarev, V. L. Korenev, M. E. Ware, M. F. Doty, T. L. Reinecke, and D. Gammon, "Optical Signatures of Coupled Quantum Dots," Science (2006).

23. D. Kim, S. G. Carter, A. Greilich, A. S. Bracker, and D. Gammon, "Ultrafast optical control of entanglement between two quantum-dot spins," Nat. Phys. 7, 223-229 (2011).

24. C. M. Chow, A. M. Ross, D. Kim, D. Gammon, A. S. Bracker, L. J. Sham, and D. G. Steel, "Nonlocal Nuclear Spin Quieting in Quantum Dot Molecules: Optically Induced Extended Two-Electron Spin Coherence Time," Phys. Rev. Lett. 117, 077403 (2016).

25. D. Press, T. D. Ladd, B. Zhang, and Y. Yamamoto, "Complete quantum control of a single quantum dot spin using ultrafast optical pulses," Nature 456, 218-221 (2008).

26. A. Delteil, Z. Sun, W. Gao, E. Togan, S. Faelt, and A. Imamoğlu, "Generation of heralded entanglement between distant hole spins," Nat. Phys. 12, 218-223 (2016).

27. S. G. Carter, T. M. Sweeney, M. Kim, C. S. Kim, D. Solenov, S. E. Economou, T. L. Reinecke, L. Yang, A. S. Bracker, and D. Gammon, "Quantum control of a spin qubit coupled to a photonic crystal cavity," Nat. Photonics 7, 329-334 (2013).

28. A. Reinhard, T. Volz, M. Winger, A. Badolato, K. J. Hennessy, E. L. Hu, and A. Imamoğlu, "Strongly correlated photons on a chip," Nat. Photonics 6, 93-96 (2012).

29. O. Gywat, H. J. Krenner, and J. Berezovsky, Spins in Optically Active Quantum Dots: Concepts and Methods (Wiley-VCH, 2010).

30. J. Y. Kim, O. Voznyy, D. Zhitomirsky, and E. H. Sargent, "25th Anniversary Article: Colloidal Quantum Dot Materials and Devices: A Quarter-Century of Advances," Adv. Mater. 25, 4986-5010 (2013).

31. K. Brunner, G. Abstreiter, G. Böhm, G. Tränkle, and G. Weimann, "Sharp-line photoluminescence of excitons localized at GaAs/AIGaAs quantum well inhomogeneities," Appl. Phys. Lett. 64, 3320-3322 (1994).

32. D. Gammon, E. S. Snow, B. V. Shanabrook, D. S. Katzer, and D. Park, "Fine Structure Splitting in the Optical Spectra of Single GaAs Quantum Dots," Phys. Rev. Lett. 76, 3005-3008 (1996).

33. R. Hanson, L. P. Kouwenhoven, J. R. Petta, S. Tarucha, and L. M. K. Vandersypen, "Spins in few-electron quantum dots," Rev. Mod. Phys. 79, 1217-1265 (2007).

34. N. Shaji, C. B. Simmons, M. Thalakulam, L. J. Klein, H. Qin, H. Luo, D. E. Savage, M. G. Lagally, A. J. Rimberg, R. Joynt, M. Friesen, R. H. Blick, S. N. Coppersmith, and M. A. Eriksson, "Spin blockade and lifetime- 
enhanced transport in a few-electron Si/SiGe double quantum dot," Nat. Phys. 4, 540-544 (2008).

35. Z. M. Wang, Self-Assembled Quantum Dots, 1st ed., Lecture Notes in Nanoscale Science and Technology No. 2195-2159 (Springer-Verlag New York, 2008), Vol. 1.

36. M. S. Skolnick and D. . J. Mowbray, "SELF-ASSEMBLED SEMICONDUCTOR QUANTUM DOTS: Fundamental Physics and Device Applications," Annu. Rev. Mater. Res. 34, 181-218 (2004).

37. I. Kamiya, I. Tanaka, O. Ohtsuki, and H. Sakaki, "Density and size control of self-assembled InAs quantum dots: preparation of very low-density dots by post-annealing," Phys. E Low-Dimens. Syst. Nanostructures 13, 1172-1175 (2002).

38. J. H. Lee, Z. M. Wang, B. L. Liang, K. A. Sablon, N. W. Strom, and G. J. Salamo, "Size and density control of InAs quantum dot ensembles on self-assembled nanostructured templates," Semicond. Sci. Technol. 21, 1547 (2006).

39. K. M. Cha, K. Shibata, and K. Hirakawa, "Single electron transport through site-controlled InAs quantum dots," Appl. Phys. Lett. 101, 223115 (2012).

40. J. H. Lee, Z. M. Wang, and G. J. Salamo, "The Control on Size and Density of InAs QDs by Droplet Epitaxy (April 2009)," IEEE Trans. Nanotechnol. 8, 431-436 (2009).

41. S. Chen, W. Li, J. Wu, Q. Jiang, M. Tang, S. Shutts, S. N. Elliott, A. Sobiesierski, A. J. Seeds, I. Ross, P. M. Smowton, and $\mathrm{H}$. Liu, "Electrically pumped continuous-wave III-V quantum dot lasers on silicon," Nat. Photonics 10, 307-311 (2016).

42. P. Martyniuk and A. Rogalski, "Quantum-dot infrared photodetectors: Status and outlook," Prog. Quantum Electron. 32, 89-120 (2008).

43. J. C. Campbell and A. Madhukar, "Quantum-Dot Infrared Photodetectors," Proc. IEEE 95, 1815-1827 (2007).

44. S. Chakrabarti, A. D. Stiff-Roberts, X. H. Su, P. Bhattacharya, G. Ariyawansa, and A. G. U. Perera, "Highperformance mid-infrared quantum dot infrared photodetectors," J. Phys. Appl. Phys. 38, 2135 (2005).

45. A. V. Kuhlmann, J. H. Prechtel, J. Houel, A. Ludwig, D. Reuter, A. D. Wieck, and R. J. Warburton, "Transformlimited single photons from a single quantum dot," Nat. Commun. 6, 8204 (2015).

46. R. J. Warburton, "Single spins in self-assembled quantum dots," Nat. Mater. 12, 483-493 (2013).

47. B. Urbaszek, X. Marie, T. Amand, O. Krebs, P. Voisin, P. Maletinsky, A. Högele, and A. Imamoglu, "Nuclear spin physics in quantum dots: An optical investigation," Rev. Mod. Phys. 85, 79-133 (2013).

48. X. Xu, W. Yao, B. Sun, D. G. Steel, A. S. Bracker, D. Gammon, and L. J. Sham, "Optically controlled locking of the nuclear field via coherent dark-state spectroscopy," Nature 459, 1105-1109 (2009).

49. B. Sun, C. M. E. Chow, D. G. Steel, A. S. Bracker, D. Gammon, and L. J. Sham, "Persistent Narrowing of Nuclear-Spin Fluctuations in InAs Quantum Dots Using Laser Excitation," Phys. Rev. Lett. 108, 187401 (2012).

50. C. Latta, A. Högele, Y. Zhao, A. N. Vamivakas, P. Maletinsky, M. Kroner, J. Dreiser, I. Carusotto, A. Badolato, D. Schuh, W. Wegscheider, M. Atature, and A. Imamoglu, "Confluence of resonant laser excitation and bidirectional quantum-dot nuclear-spin polarization," Nat. Phys. 5, 758-763 (2009).

51. I. T. Vink, K. C. Nowack, F. H. L. Koppens, J. Danon, Y. V. Nazarov, and L. M. K. Vandersypen, "Locking electron spins into magnetic resonance by electron-nuclear feedback," Nat. Phys. 5, 764-768 (2009).

52. A. Högele, M. Kroner, C. Latta, M. Claassen, I. Carusotto, C. Bulutay, and A. Imamoglu, "Dynamic Nuclear Spin Polarization in the Resonant Laser Excitation of an InGaAs Quantum Dot," Phys. Rev. Lett. 108, 
197403 (2012).

53. H. D. Robinson and B. B. Goldberg, "Light-induced spectral diffusion in single self-assembled quantum dots," Phys. Rev. B 61, R5086-R5089 (2000).

54. J. Houel, A. V. Kuhlmann, L. Greuter, F. Xue, M. Poggio, B. D. Gerardot, P. A. Dalgarno, A. Badolato, P. M. Petroff, A. Ludwig, D. Reuter, A. D. Wieck, and R. J. Warburton, "Probing Single-Charge Fluctuations at a GaAs/AlAs Interface Using Laser Spectroscopy on a Nearby InGaAs Quantum Dot," Phys. Rev. Lett. 108, 107401 (2012).

55. C. Matthiesen, M. J. Stanley, M. Hugues, E. Clarke, and M. Atatüre, "Full counting statistics of quantum dot resonance fluorescence," Sci. Rep. 4, (2014).

56. C. K. Hong, Z. Y. Ou, and L. Mandel, "Measurement of subpicosecond time intervals between two photons by interference," Phys. Rev. Lett. 59, 2044-2046 (1987).

57. P. Kok, "Linear optical quantum computing with photonic qubits," Rev. Mod. Phys. 79, 135-174 (2007).

58. A. Berthelot, I. Favero, G. Cassabois, C. Voisin, C. Delalande, P. Roussignol, R. Ferreira, and J. M. Gérard, "Unconventional motional narrowing in the optical spectrum of a semiconductor quantum dot," Nat. Phys. 2, 759-764 (2006).

59. I. Favero, "Temperature dependence of the zero-phonon linewidth in quantum dots: An effect of the fluctuating environment," Phys. Rev. B 75, (2007).

60. C. Arnold, V. Loo, A. Lemaître, I. Sagnes, O. Krebs, P. Voisin, P. Senellart, and L. Lanco, "Cavity-Enhanced Real-Time Monitoring of Single-Charge Jumps at the Microsecond Time Scale," Phys. Rev. X 4, 021004 (2014).

61. H. S. Nguyen, G. Sallen, M. Abbarchi, R. Ferreira, C. Voisin, P. Roussignol, G. Cassabois, and C. Diederichs, "Photoneutralization and slow capture of carriers in quantum dots probed by resonant excitation spectroscopy," Phys. Rev. B 87, 115305 (2013).

62. H. S. Nguyen, G. Sallen, C. Voisin, P. Roussignol, C. Diederichs, and G. Cassabois, "Optically Gated Resonant Emission of Single Quantum Dots," Phys. Rev. Lett. 108, 057401 (2012).

63. E. W. Bogaart, J. E. M. Haverkort, T. Mano, T. van Lippen, R. Nötzel, and J. H. Wolter, "Role of the continuum background for carrier relaxation in InAs quantum dots," Phys. Rev. B 72, 195301 (2005).

64. H. Kurtze, J. Seebeck, P. Gartner, D. R. Yakovlev, D. Reuter, A. D. Wieck, M. Bayer, and F. Jahnke, "Carrier relaxation dynamics in self-assembled semiconductor quantum dots," Phys. Rev. B 80, 235319 (2009).

65. Bogaart, EW Erik, Lenstra, D Daan, Koopmans, B Bert, Haverkort, JEM Jos, and Technische Universiteit Eindhoven, "Transient differential reflection spectroscopy on quantum dot nanostructures," Technische Universiteit Eindhoven (2006).

66. A. Muller, E. B. Flagg, P. Bianucci, X. Y. Wang, D. G. Deppe, W. Ma, J. Zhang, G. J. Salamo, M. Xiao, and C. K. Shih, "Resonance Fluorescence from a Coherently Driven Semiconductor Quantum Dot in a Cavity," Phys. Rev. Lett. 99, 187402 (2007).

67. X. Xu, B. Sun, P. R. Berman, D. G. Steel, A. S. Bracker, D. Gammon, and L. J. Sham, "Coherent Optical Spectroscopy of a Strongly Driven Quantum Dot," Science 317, 929-932 (2007).

68. K. Kuroda, T. Kuroda, K. Watanabe, T. Mano, K. Sakoda, G. Kido, and N. Koguchi, "Final-state readout of exciton qubits by observing resonantly excited photoluminescence in quantum dots," Appl. Phys. Lett. 90, 051909 (2007).

69. R. Melet, V. Voliotis, A. Enderlin, D. Roditchev, X. L. Wang, T. Guillet, and R. Grousson, "Resonant excitonic 
emission of a single quantum dot in the Rabi regime," Phys. Rev. B 78, 073301 (2008).

70. E. B. Flagg, A. Muller, J. W. Robertson, S. Founta, D. G. Deppe, M. Xiao, W. Ma, G. J. Salamo, and C. K. Shih, "Resonantly driven coherent oscillations in a solid-state quantum emitter," Nat. Phys. 5, 203-207 (2009).

71. K. Konthasinghe, J. Walker, M. Peiris, C. K. Shih, Y. Yu, M. F. Li, J. F. He, L. J. Wang, H. Q. Ni, Z. C. Niu, and A. Muller, "Coherent versus incoherent light scattering from a quantum dot," Phys. Rev. B 85, 235315 (2012).

72. H. S. Nguyen, G. Sallen, C. Voisin, P. Roussignol, C. Diederichs, and G. Cassabois, "Ultra-coherent single photon source," Appl. Phys. Lett. 99, 261904 (2011).

73. W. Heitler, The Quantum Theory of Radiation, 3 edition, Dover Books on Physics (Dover Publications, 2010).

74. C. Matthiesen, M. Geller, C. H. H. Schulte, C. Le Gall, J. Hansom, Z. Li, M. Hugues, E. Clarke, and M. Atatüre, "Phase-locked indistinguishable photons with synthesized waveforms from a solid-state source," Nat. Commun. 4, 1600 (2013).

75. S. Arroyo-Camejo, A. Lazariev, S. W. Hell, and G. Balasubramanian, "Room temperature high-fidelity holonomic single-qubit gate on a solid-state spin," Nat. Commun. 5, 4870 (2014).

76. P. Siyushev, K. Xia, R. Reuter, M. Jamali, N. Zhao, N. Yang, C. Duan, N. Kukharchyk, A. D. Wieck, R. Kolesov, and J. Wrachtrup, "Coherent properties of single rare-earth spin qubits," Nat. Commun. 5, 3895 (2014).

77. D. Leonard, K. Pond, and P. M. Petroff, "Critical layer thickness for self-assembled InAs islands on GaAs," Phys. Rev. B 50, 11687-11692 (1994).

78. N. P. Kobayashi, T. R. Ramachandran, P. Chen, and A. Madhukar, "In situ, atomic force microscope studies of the evolution of InAs three-dimensional islands on GaAs(001)," Appl. Phys. Lett. 68, 3299-3301 (1996).

79. A. S. Bhatti, M. Grassi Alessi, M. Capizzi, P. Frigeri, and S. Franchi, "Optical spectroscopy of quasimonolayer InAs at the onset of quantum-dot nucleation," Phys. Rev. B 60, 2592-2598 (1999).

80. T. Walther, A. G. Cullis, D. J. Norris, and M. Hopkinson, "Nature of the Stranski-Krastanow Transition during Epitaxy of InGaAs on GaAs," Phys. Rev. Lett. 86, 2381-2384 (2001).

81. A. G. Cullis, D. J. Norris, T. Walther, M. A. Migliorato, and M. Hopkinson, "Stranski-Krastanow transition and epitaxial island growth," Phys. Rev. B 66, 081305 (2002).

82. I. Kegel, T. H. Metzger, A. Lorke, J. Peisl, J. Stangl, G. Bauer, J. M. García, and P. M. Petroff, "NanometerScale Resolution of Strain and Interdiffusion in Self-Assembled InAs/GaAs Quantum Dots," Phys. Rev. Lett. 85, 1694-1697 (2000).

83. I. Kegel, T. H. Metzger, A. Lorke, J. Peisl, J. Stangl, G. Bauer, K. Nordlund, W. V. Schoenfeld, and P. M. Petroff, "Determination of strain fields and composition of self-organized quantum dots using $\mathrm{x}$-ray diffraction," Phys. Rev. B 63, 035318 (2001).

84. E. Placidi, F. Arciprete, V. Sessi, M. Fanfoni, F. Patella, and A. Balzarotti, "Step erosion during nucleation of InAs/GaAs(001) quantum dots," Appl. Phys. Lett. 86, 241913 (2005).

85. D. Leonard, M. Krishnamurthy, C. M. Reaves, S. P. Denbaars, and P. M. Petroff, "Direct formation of quantum-sized dots from uniform coherent islands of InGaAs on GaAs surfaces," Appl. Phys. Lett. 63, 3203-3205 (1993).

86. F. Patella, S. Nufris, F. Arciprete, M. Fanfoni, E. Placidi, A. Sgarlata, and A. Balzarotti, "Tracing the two- to three-dimensional transition in the InAs/GaAs(001) heteroepitaxial growth," Phys. Rev. B 67, 205308 (2003). 
87. F. Patella, A. Sgarlata, F. Arciprete, S. Nufris, P. D. Szkutnik, E. Placidi, M. Fanfoni, N. Motta, and A. Balzarotti, "Self-assembly of InAs and Si/Ge quantum dots on structured surfaces," J. Phys. Condens. Matter 16, S1503 (2004).

88. Michler, Single Quantum Dots - Fundamentals, Applications, and New Concepts, Topics in Applied Physics (Springer Berlin Heidelberg, 2003), Vol. 90.

89. D. T. F. Marple, "Refractive Index of GaAs," J. Appl. Phys. 35, 1241-1242 (1964).

90. J. Talghader and J. S. Smith, "Thermal dependence of the refractive index of GaAs and AlAs measured using semiconductor multilayer optical cavities," Appl. Phys. Lett. 66, 335-337 (1995).

91. D. Campi and C. Papuzza, "Refractive index dispersion in group IV and binary III-V semiconductors: Comparison of calculated and experimental values," J. Appl. Phys. 57, 1305-1310 (1985).

92. R. E. Fern and A. Onton, "Refractive Index of AlAs," J. Appl. Phys. 42, 3499-3500 (1971).

93. P. Yeh, Optical Waves in Layered Media, 2nd ed., Wiley Series in Pure and Applied Optics (WileyInterscience, 2005).

94. H. K. H. Choy, "Design and Fabrication of Distributed Bragg Reflectors for Vertical-Cavity Surface-Emitting Lasers," Master of Science, Massachusetts Institute of Technology (1998).

95. H. Benisty, H. De Neve, and C. Weisbuch, "Impact of planar microcavity effects on light extraction-Part I: basic concepts and analytical trends," IEEE J. Quantum Electron. 34, 1612-1631 (1998).

96. G. Ramon, "Emission characteristics of quantum dots in planar microcavities," Phys. Rev. B 73, (2006).

97. A. Schliwa, M. Winkelnkemper, and D. Bimberg, "Impact of size, shape, and composition on piezoelectric effects and electronic properties of InGaAs/GaAs quantum dots," Phys. Rev. B 76, 205324 (2007).

98. O. Stier, M. Grundmann, and D. Bimberg, "Electronic and optical properties of strained quantum dots modeled by 8-band k.p theory," Phys. Rev. B 59, 5688-5701 (1999).

99. M. Grundmann, O. Stier, and D. Bimberg, "InAs/GaAs pyramidal quantum dots: Strain distribution, optical phonons, and electronic structure," Phys. Rev. B 52, 11969-11981 (1995).

100. J. A. Barker and E. P. O'Reilly, "Theoretical analysis of electron-hole alignment in InAs-GaAs quantum dots," Phys. Rev. B 61, 13840-13851 (2000).

101. I. Filikhin, V. M. Suslov, M. Wu, and B. Vlahovic, "InGaAs/GaAs quantum dots within an effective approach," Phys. E Low-Dimens. Syst. Nanostructures 41, 1358-1363 (2009).

102. I. Saïdi, K. Sellami, M. Yahyaoui, C. Testelin, and K. Boujdaria, "Electron and hole energy levels in InAs/GaAs quantum dots: Size and magnetic field effects," J. Appl. Phys. 109, 033703 (2011).

103. R. J. Warburton, C. Schulhauser, D. Haft, C. Schäflein, K. Karrai, J. M. Garcia, W. Schoenfeld, and P. M. Petroff, "Giant permanent dipole moments of excitons in semiconductor nanostructures," Phys. Rev. B 65, 113303 (2002).

104. H. Jiang and J. Singh, "Strain distribution and electronic spectra of InAs/GaAs self-assembled dots: An eight-band study," Phys. Rev. B 56, 4696-4701 (1997).

105. S. Lee, O. L. Lazarenkova, P. von Allmen, F. Oyafuso, and G. Klimeck, "Effect of wetting layers on the strain and electronic structure of InAs self-assembled quantum dots," Phys. Rev. B 70, 125307 (2004).

106. G. A. Narvaez, G. Bester, and A. Zunger, "Carrier relaxation mechanisms in self-assembled (In,Ga)As/GaAs quantum dots: Efficient P->S Auger relaxation of electrons," Phys. Rev. B 74, 075403 (2006).

107. H. W. van Kesteren, E. C. Cosman, W. A. J. A. van der Poel, and C. T. Foxon, "Fine structure of excitons in type-II GaAs/AlAs quantum wells," Phys. Rev. B 41, 5283-5292 (1990). 
108. L. C. Andreani and F. Bassani, "Exchange interaction and polariton effects in quantum-well excitons," Phys. Rev. B 41, 7536-7544 (1990).

109. E. Blackwood, M. J. Snelling, R. T. Harley, S. R. Andrews, and C. T. B. Foxon, "Exchange interaction of excitons in GaAs heterostructures," Phys. Rev. B 50, 14246-14254 (1994).

110. M. Bayer, G. Ortner, O. Stern, A. Kuther, A. A. Gorbunov, A. Forchel, P. Hawrylak, S. Fafard, K. Hinzer, T. L. Reinecke, S. N. Walck, J. P. Reithmaier, F. Klopf, and F. Schäfer, "Fine structure of neutral and charged excitons in self-assembled In(Ga)As/(Al)GaAs quantum dots," Phys. Rev. B 65, 195315 (2002).

111. J. M. Luttinger and W. Kohn, "Motion of Electrons and Holes in Perturbed Periodic Fields," Phys. Rev. 97, 869-883 (1955).

112. S. Rodt, A. Schliwa, K. Pötschke, F. Guffarth, and D. Bimberg, "Correlation of structural and few-particle properties of self-organized InAs/GaAs quantum dots," Phys. Rev. B 71, 155325 (2005).

113. U. W. Pohl, K. Pötschke, A. Schliwa, M. B. Lifshits, V. A. Shchukin, D. E. Jesson, and D. Bimberg, "Formation and evolution of multimodal size distributions of InAs/GaAs quantum dots," Phys. E Low-Dimens. Syst. Nanostructures 32, 9-13 (2006).

114. J. M. Smith, P. A. Dalgarno, R. J. Warburton, A. O. Govorov, K. Karrai, B. D. Gerardot, and P. M. Petroff, "Voltage Control of the Spin Dynamics of an Exciton in a Semiconductor Quantum Dot," Phys. Rev. Lett. 94, 197402 (2005).

115. X. Xu, B. Sun, P. R. Berman, D. G. Steel, A. S. Bracker, D. Gammon, and L. J. Sham, "Coherent population trapping of an electron spin in a single negatively charged quantum dot," Nat. Phys. 4, 692-695 (2008).

116. D. Brunner, B. D. Gerardot, P. A. Dalgarno, G. Wüst, K. Karrai, N. G. Stoltz, P. M. Petroff, and R. J. Warburton, "A Coherent Single-Hole Spin in a Semiconductor," Science 325, 70-72 (2009).

117. D. Press, K. De Greve, P. L. McMahon, T. D. Ladd, B. Friess, C. Schneider, M. Kamp, S. Höfling, A. Forchel, and Y. Yamamoto, "Ultrafast optical spin echo in a single quantum dot," Nat. Photonics 4, 367-370 (2010).

118. K. De Greve, P. L. McMahon, D. Press, T. D. Ladd, D. Bisping, C. Schneider, M. Kamp, L. Worschech, S. Höfling, A. Forchel, and Y. Yamamoto, "Ultrafast coherent control and suppressed nuclear feedback of a single quantum dot hole qubit," Nat. Phys. 7, 872-878 (2011).

119. M. G. Littman and H. J. Metcalf, "Spectrally narrow pulsed dye laser without beam expander," Appl. Opt. 17, 2224-2227 (1978).

120. K. Liu and M. G. Littman, "Novel geometry for single-mode scanning of tunable lasers," Opt. Lett. 6, 117118 (1981).

121. C. J. Hawthorn, K. P. Weber, and R. E. Scholten, "Littrow configuration tunable external cavity diode laser with fixed direction output beam," Rev. Sci. Instrum. 72, 4477-4479 (2001).

122. A. N. Vamivakas, Y. Zhao, C.-Y. Lu, and M. Atatüre, "Spin-resolved quantum-dot resonance fluorescence," Nat. Phys. 5, 198-202 (2009).

123. A. V. Kuhlmann, J. Houel, D. Brunner, A. Ludwig, D. Reuter, A. D. Wieck, and R. J. Warburton, "A dark-field microscope for background-free detection of resonance fluorescence from single semiconductor quantum dots operating in a set-and-forget mode," Rev. Sci. Instrum. 84, 073905 (2013).

124. P. Terrier, J. M. Charbois, and V. Devlaminck, "Fast-axis orientation dependence on driving voltage for a Stokes polarimeter based on concrete liquid-crystal variable retarders," Appl. Opt. 49, 4278 (2010).

125. M. Davanço, C. S. Hellberg, S. Ates, A. Badolato, and K. Srinivasan, "Multiple time scale blinking in InAs quantum dot single-photon sources," Phys. Rev. B 89, 161303 (2014). 
126. D. Magatti and F. Ferri, "Fast multi-tau real-time software correlator for dynamic light scattering," Appl. Opt. 40, 4011 (2001).

127. J. Ramírez, S. K. Sukumaran, B. Vorselaars, and A. E. Likhtman, "Efficient on the fly calculation of time correlation functions in computer simulations," J. Chem. Phys. 133, 154103 (2010).

128. T. A. Laurence, S. Fore, and T. Huser, "Fast, flexible algorithm for calculating photon correlations," Opt. Lett. 31, 829 (2006).

129. M. J. Culbertson and D. L. Burden, "A distributed algorithm for multi-tau autocorrelation," Rev. Sci. Instrum. 78, 044102 (2007).

130. Y. Toda, O. Moriwaki, M. Nishioka, and Y. Arakawa, "Efficient Carrier Relaxation Mechanism in InGaAs/GaAs Self-Assembled Quantum Dots Based on the Existence of Continuum States," Phys. Rev. Lett. 82, 4114-4117 (1999).

131. D. Morris, N. Perret, and S. Fafard, "Carrier energy relaxation by means of Auger processes in InAs/GaAs self-assembled quantum dots," Appl. Phys. Lett. 75, 3593-3595 (1999).

132. S. Sanguinetti, K. Watanabe, T. Tateno, M. Wakaki, N. Koguchi, T. Kuroda, F. Minami, and M. Gurioli, "Role of the wetting layer in the carrier relaxation in quantum dots," Appl. Phys. Lett. 81, 613-615 (2002).

133. E. L. Ivchenko, "Fine Structure of Excitonic Levels in Semiconductor Nanostructures," Phys. Status Solidi A 164, 487-492 (1997).

134. H. Tong and M. W. Wu, "Theory of excitons in cubic III-V semiconductor GaAs, InAs and GaN quantum dots: Fine structure and spin relaxation," Phys. Rev. B 83, 235323 (2011).

135. B. R. Mollow, "Power Spectrum of Light Scattered by Two-Level Systems," Phys. Rev. 188, 1969-1975 (1969).

136. F. Y. Wu, R. E. Grove, and S. Ezekiel, "Investigation of the Spectrum of Resonance Fluorescence Induced by a Monochromatic Field," Phys. Rev. Lett. 35, 1426-1429 (1975).

137. M. Metcalfe, G. S. Solomon, and J. Lawall, "Heterodyne measurement of resonant elastic scattering from epitaxial quantum dots," Appl. Phys. Lett. 102, 231114 (2013).

138. N. H. Bonadeo, J. Erland, D. Gammon, D. Park, D. S. Katzer, and D. G. Steel, "Coherent Optical Control of the Quantum State of a Single Quantum Dot," Science 282, 1473-1476 (1998).

139. D. Chen, G. R. Lander, G. S. Solomon, and E. B. Flagg, "Polarization-Dependent Interference of Coherent Scattering from Orthogonal Dipole Moments of a Resonantly Excited Quantum Dot," Phys. Rev. Lett. 118, 037401 (2017).

140. R. Loudon, The Quantum Theory of Light, 3rd ed. (OUP Oxford University Press, 2000).

141. M. O. Scully and M. S. Zubairy, Quantum Optics (Cambridge University Press, 1997).

142. G. Lindblad, "On the generators of quantum dynamical semigroups," Commun. Math. Phys. 48, 119-130 (1976).

143. H. J. Carmichael, Statistical Methods in Quantum Optics 1_Master Equations and Fokker-Planck Equations, Second Corrected Printing (Springer Berlin Heidelberg, 1999).

144. S.-Y. Lu and R. A. Chipman, "Interpretation of Mueller matrices based on polar decomposition," J. Opt. Soc. Am. A 13, 1106 (1996).

145. D. Chen, G. R. Lander, K. S. Krowpman, G. S. Solomon, and E. B. Flagg, "Characterization of the local charge environment of a single quantum dot via resonance fluorescence," Phys. Rev. B 93, 115307 (2016).

146. L. Allen and J. H. Eberly, Optical Resonance and Two Level Atoms (Wiley, New York, 1975). 
147. G. Bester and A. Zunger, "Cylindrically shaped zinc-blende semiconductor quantum dots do not have cylindrical symmetry: Atomistic symmetry, atomic relaxation, and piezoelectric effects," Phys. Rev. B 71, 045318 (2005).

148. J.-W. Luo, G. Bester, and A. Zunger, "Supercoupling between heavy-hole and light-hole states in nanostructures," Phys. Rev. B 92, 165301 (2015).

149. D. N. Krizhanovskii, A. Ebbens, A. I. Tartakovskii, F. Pulizzi, T. Wright, M. S. Skolnick, and M. Hopkinson, "Individual neutral and charged $\$\{\backslash$ mathrm $\{\mid \mathrm{n}\}\} \_\{x\}\{\backslash \text { mathrm }\{\text { Ga }\}\}_{-}\{1 \backslash$ ensuremath $\{-\} \mathrm{x}\} \backslash$ mathrm $\{$ As $\} \backslash$ text $\{\backslash$ ensuremath $\{-\}\} \backslash$ mathrm $\{$ Ga $\} \backslash$ mathrm\{As\}\$ quantum dots with strong in-plane optical anisotropy," Phys. Rev. B 72, 161312 (2005).

150. J. Dreiser, M. Atatüre, C. Galland, T. Müller, A. Badolato, and A. Imamoglu, "Optical investigations of quantum dot spin dynamics as a function of external electric and magnetic fields," Phys. Rev. B 77, 075317 (2008).

151. T. Belhadj, T. Amand, A. Kunold, C.-M. Simon, T. Kuroda, M. Abbarchi, T. Mano, K. Sakoda, S. Kunz, X. Marie, and B. Urbaszek, "Impact of heavy hole-light hole coupling on optical selection rules in GaAs quantum dots," Appl. Phys. Lett. 97, 051111 (2010).

152. C.-Y. Lu, Y. Zhao, A. N. Vamivakas, C. Matthiesen, S. Fält, A. Badolato, and M. Atatüre, "Direct measurement of spin dynamics in InAs/GaAs quantum dots using time-resolved resonance fluorescence," Phys. Rev. B 81, 035332 (2010).

153. T. Unold, K. Mueller, C. Lienau, T. Elsaesser, and A. D. Wieck, "Optical Stark Effect in a Quantum Dot: Ultrafast Control of Single Exciton Polarizations," Phys. Rev. Lett. 92, 157401 (2004).

154. A. Muller, W. Fang, J. Lawall, and G. S. Solomon, "Emission Spectrum of a Dressed Exciton-Biexciton Complex in a Semiconductor Quantum Dot," Phys. Rev. Lett. 101, 027401 (2008).

155. A. Muller, W. Fang, J. Lawall, and G. S. Solomon, "Creating Polarization-Entangled Photon Pairs from a Semiconductor Quantum Dot Using the Optical Stark Effect," Phys. Rev. Lett. 103, 217402 (2009).

156. L. Onsager, "Reciprocal Relations in Irreversible Processes. II.," Phys. Rev. 38, 2265-2279 (1931).

157. M. Lax, "Formal Theory of Quantum Fluctuations from a Driven State," Phys. Rev. 129, 2342-2348 (1963).

158. H.-P. Breuer and F. Petruccione, The Theory of Open Quantum Systems (Oxford University Press, 2007).

159. C. Gardiner and P. Zoller, Quantum Noise, 3rd ed., Springer Series in Synergetics No. 0172-7389 (SpringerVerlag Berlin Heidelberg, 2004).

160. C. Cohen-Tannoudji and S. Reynaud, "Dressed-atom description of resonance fluorescence and absorption spectra of a multi-level atom in an intense laser beam," J. Phys. B At. Mol. Phys. 10, 345 (1977).

161. R.-C. Ge, S. Weiler, A. Ulhaq, S. M. Ulrich, M. Jetter, P. Michler, and S. Hughes, "Mollow quintuplets from coherently excited quantum dots," Opt. Lett. 38, 1691 (2013).

162. H. Paul, "Photon antibunching," Rev. Mod. Phys. 54, 1061-1102 (1982).

163. M. Fox, Quantum Optics_an Introduction, 1st ed., Oxford Master Series in Physics No. Book 15 (Oxford University Press, 2006).

164. P. W. Fry, I. E. Itskevich, D. J. Mowbray, M. S. Skolnick, J. J. Finley, J. A. Barker, E. P. O’Reilly, L. R. Wilson, I. A. Larkin, P. A. Maksym, M. Hopkinson, M. Al-Khafaji, J. P. R. David, A. G. Cullis, G. Hill, and J. C. Clark, "Inverted Electron-Hole Alignment in InAs-GaAs Self-Assembled Quantum Dots," Phys. Rev. Lett. 84, 733736 (2000).

165. M. Abbarchi, F. Troiani, C. Mastrandrea, G. Goldoni, T. Kuroda, T. Mano, K. Sakoda, N. Koguchi, S. 
Sanguinetti, A. Vinattieri, and M. Gurioli, "Spectral diffusion and line broadening in single self-assembled GaAs / AlGaAs quantum dot photoluminescence," Appl. Phys. Lett. 93, 162101 (2008).

166. M. Abbarchi, T. Kuroda, T. Mano, M. Gurioli, and K. Sakoda, "Bunched photon statistics of the spectrally diffusive photoluminescence of single self-assembled GaAs quantum dots," Phys. Rev. B 86, 115330 (2012).

167. A. P. Beyler, L. F. Marshall, J. Cui, X. Brokmann, and M. G. Bawendi, "Direct Observation of Rapid Discrete Spectral Dynamics in Single Colloidal CdSe-CdS Core-Shell Quantum Dots," Phys. Rev. Lett. 111, 177401 (2013).

168. S. Unsleber, D. P. S. McCutcheon, M. Dambach, M. Lermer, N. Gregersen, S. Höfling, J. Mørk, C. Schneider, and M. Kamp, "Two-photon interference from a quantum dot microcavity: Persistent pure dephasing and suppression of time jitter," Phys. Rev. B 91, 075413 (2015).

169. X. Ding, Y. He, Z.-C. Duan, N. Gregersen, M.-C. Chen, S. Unsleber, S. Maier, C. Schneider, M. Kamp, S. Höfling, C.-Y. Lu, and J.-W. Pan, "On-Demand Single Photons with High Extraction Efficiency and NearUnity Indistinguishability from a Resonantly Driven Quantum Dot in a Micropillar," Phys. Rev. Lett. 116, 020401 (2016).

170. R. J. Warburton, C. Schäflein, D. Haft, F. Bickel, A. Lorke, K. Karrai, J. M. Garcia, W. Schoenfeld, and P. M. Petroff, "Optical emission from a charge-tunable quantum ring," Nature 405, 926-929 (2000).

171. M. Baier, F. Findeis, A. Zrenner, M. Bichler, and G. Abstreiter, "Optical spectroscopy of charged excitons in single quantum dot photodiodes," Phys. Rev. B 64, 195326 (2001).

172. J. J. Finley, P. W. Fry, A. D. Ashmore, A. Lemaître, A. I. Tartakovskii, R. Oulton, D. J. Mowbray, M. S. Skolnick, M. Hopkinson, P. D. Buckle, and P. A. Maksym, "Observation of multicharged excitons and biexcitons in a single InGaAs quantum dot," Phys. Rev. B 63, 161305 (2001).

173. M. Hauck, F. Seilmeier, S. E. Beavan, A. Badolato, P. M. Petroff, and A. Högele, "Locating environmental charge impurities with confluent laser spectroscopy of multiple quantum dots," Phys. Rev. B 90, 235306 (2014).

174. B. D. Gerardot, S. Seidl, P. A. Dalgarno, R. J. Warburton, D. Granados, J. M. Garcia, K. Kowalik, O. Krebs, K. Karrai, A. Badolato, and P. M. Petroff, "Manipulating exciton fine structure in quantum dots with a lateral electric field," Appl. Phys. Lett. 90, 041101 (2007).

175. A. Zolotaryov, A. Schramm, C. Heyn, and W. Hansen, "InAs-coverage dependence of self-assembled quantum dot size, composition, and density," Appl. Phys. Lett. 91, 083107 (2007).

176. H. Nakajima, H. Kumano, H. lijima, S. Odashima, and I. Suemune, "Carrier-transfer dynamics between neutral and charged excitonic states in a single quantum dot probed with second-order photon correlation measurements," Phys. Rev. B 88, 045324 (2013).

177. H. J. Kimble, "The quantum internet," Nature 453, 1023-1030 (2008). 


\section{Appendix}

\section{Appendix A2.1 Emission spectrum measured with spectrometer}

3Even though we cannot resolve the fine energy structure of a neutral quantum dot by using the spectrometer (resolution $35 \mathrm{GHz}$ ), it is capable to distinguish the emissions from different exciton species of a single QD. Generally, the emission lines from the other three exciton complexes (negative trion $\left(\mathrm{X}^{-}\right)$, positive trion $\left(\mathrm{X}^{+}\right)$ and biexciton $(\mathrm{XX})$ ) lie within $\pm 15 \mathrm{meV}$ range around the neutral exciton line $\left(\mathrm{X}^{0}\right)$ [112], i.e., $\pm 10 \mathrm{~nm}$ around $928 \mathrm{~nm}$. Figure A2.1 shows the emission spectra of QD1 (a-b) and QD2 (c-d) under solely above-band excitation. The opening of the slit (15) in Figure 2.6 is $120 \mu \mathrm{m}$ for QD1 and $30 \mu \mathrm{m}$ for QD2, respectively. The bottom line plots in Figure A2.1(b) and (d) are the extracted PL intensity along the grey line in the corresponding top images. Even though evident side peaks appear around $\mathrm{X}^{0}$ emission line in (b) and (d), they are not the emissions from other exciton states of the same QD, evidenced by carefully examining the CCD images in (a) and (c). Following the same method, we carefully checked the whole range of $\pm 10 \mathrm{~nm}$ of $\mathrm{X}^{0}$ line, and find no peaks corresponding to trion or biexciton emissions from QD1 or QD2.
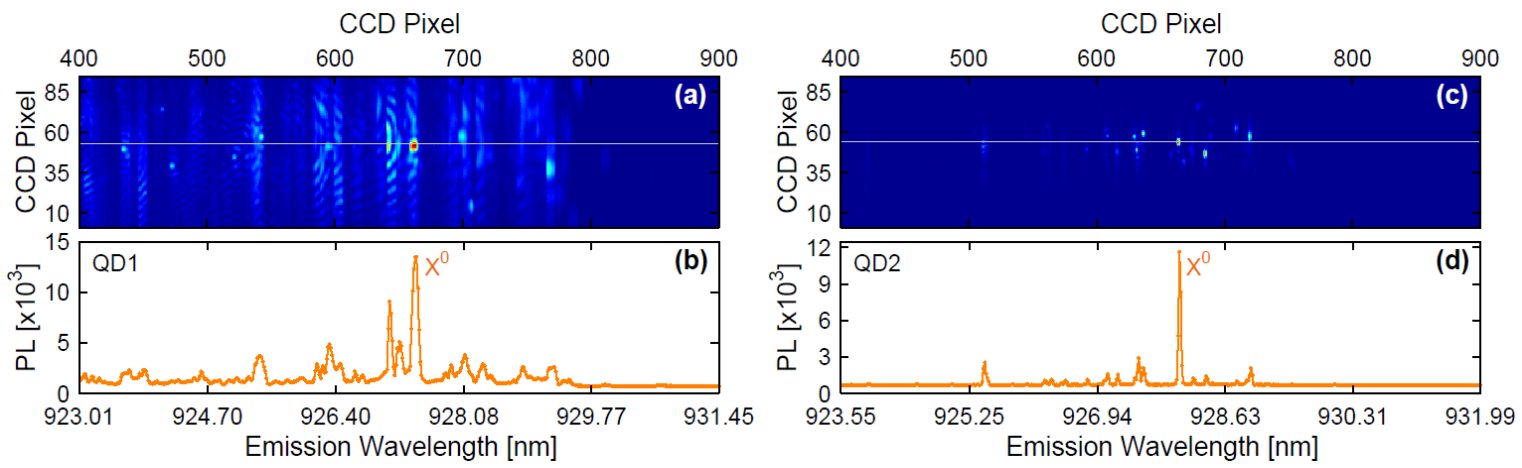

Figure A2.1 The emission spectra of QD1 (a-b) and QD2 (c-d) under solely above band-gap excitation. The frame containing QD1, (a), is taken with an excitation power of $215 \mathrm{nW}, 80 \mathrm{x}$ magnification, $120 \mu \mathrm{m}$ open slit, and an exposure time of $10 \mathrm{~min}$. The frame containing QD2, (c), is taken with an excitation power of 704 $\mathrm{nW}, 40 \mathrm{x}$ magnification, $30 \mu \mathrm{m}$ open slit, and an exposure time of 5 secs. The spectrum in (b) and (d) are the extracted PL intensity along the grey lines in (a) and (b), respectively. The peaks labeled by $\mathrm{X}^{0}$ in (b) and (d) correspond to the neutral exciton emission of QD1 and QD2, respectively. 


\section{Appendix A2.2 Determining optimal LCVR voltages}

Considering the voltage-dependent shift of the fast axis of each LCVR, it is hard to predict or calculate the best voltages for measuring the projected intensities along X-, Y-, D-, A- and L-, R-polarization. However, it is still possible to pin down these parameters experimentally by brute force mapping the transmission intensity for all possible voltage combinations of two LCVRs. Figure A2.2 shows the experimental setup for this 2D mapping. A continuous-wave laser in a specific polarization, controlled by a linear polarizer, is incident onto two LCVRs and passes through a linear analyzer before being detected by a photodiode. Each map is defined by the chosen polarizations for the polarizer and the analyzer. Ideally, six maps with incident polarization along X, Y, D, A and $\mathrm{L}$, R-direction and fixed analyzer polarization $(\mathrm{X})$ are preferred to being collected, because these combinations (XX, YX, DX, AX, and LX, RX) are the targeted polarizations for PL measurement. However, it is difficult to produce a perfect circularly polarized light with a quarter-wave-plate (QWP) at a general wavelength. In fact, the phase retardance of our QWP is off by at least 7\% from $\lambda / 4$ at $928 \mathrm{~nm}$ according the experimental measurement. Hence, instead of LX and RX maps, we collect two new maps with the polarizer-analyzer combination oriented in DD and AD directions. Figure A2.3 shows these six maps (XX, YX, DX, AX, and DD, AD) with each normalized to its own maximum intensity.

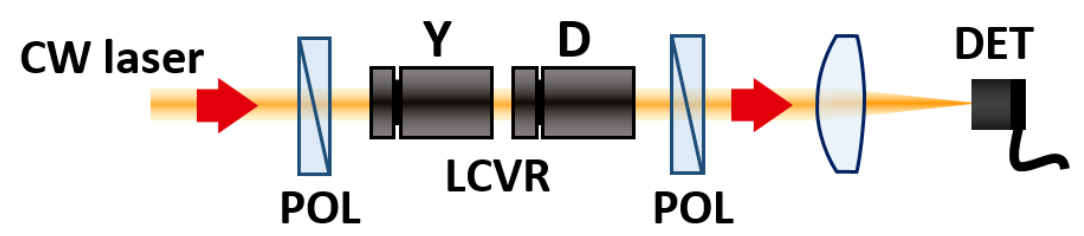

Figure A2.2 Experiment setup for determining the optimal LCVR voltages. The fast axis of two LCVRs are aligned to $Y$ and D direction, respectively. The $\mathrm{cw}$ laser is tuned to $928.39 \mathrm{~nm}$. POL is the linear polarizer, and DET is the voltage-biased photodiode.

The optimal voltages can be pinpointed by searching for the location in these maps that simultaneously complies with the following requirements:

\begin{tabular}{c|cccccc} 
Target & Map XX & Map YX & Map DX & Map AX & Map DD & Map AD \\
\hline X & 1 & 0 & $50 \%$ & $50 \%$ & 1 & 0 \\
Y & 0 & 1 & $50 \%$ & $50 \%$ & 1 & 0 \\
D & $50 \%$ & $50 \%$ & 1 & 0 & $50 \%$ & $50 \%$ \\
A & $50 \%$ & $50 \%$ & 0 & 1 & $50 \%$ & $50 \%$ \\
R & $50 \%$ & $50 \%$ & $50 \%$ & $50 \%$ & 0 & 1 \\
L & $50 \%$ & $50 \%$ & $50 \%$ & $50 \%$ & 1 & 0
\end{tabular}

Each row lists six values of expected intensity (each from one map) if the voltages of LCVRs are optimized for measuring the PL projection along the targeted polarizations listed in the leftmost column. For example, the optimal location for measuring X-polarization component would be the voltage pair that gives the maximum intensity in XX and DD map, the minimum intensity in YX and AD map, and 50\% intensity in DX and AX map. 
Therefore, we can pinpoint the optimal voltages by searching through these six maps according to the listed features. In fact, these requirements are too ideal and harsh for experimentalists to follow, since, for example, any experimental noise or errors would lift the minimum intensity from zero. Practically, we neglect the requirement on minimum intensity, and for the maximum intensity we consider the range $99 \%$ to 1 . We plot all these requirements in contours in one figure for an easier comparison, as shown in Figure A2.4. The final optimal voltage pairs are depicted by the black dots, which are generally consistent with the above requirements. We noticed that the optimal voltages are slightly different from one run to another (probably due to the intrinsic property of the liquid crystal). Therefore, we repeat the above mapping within a smaller voltage range a couple of times and conclude that the black dots are the best locations.

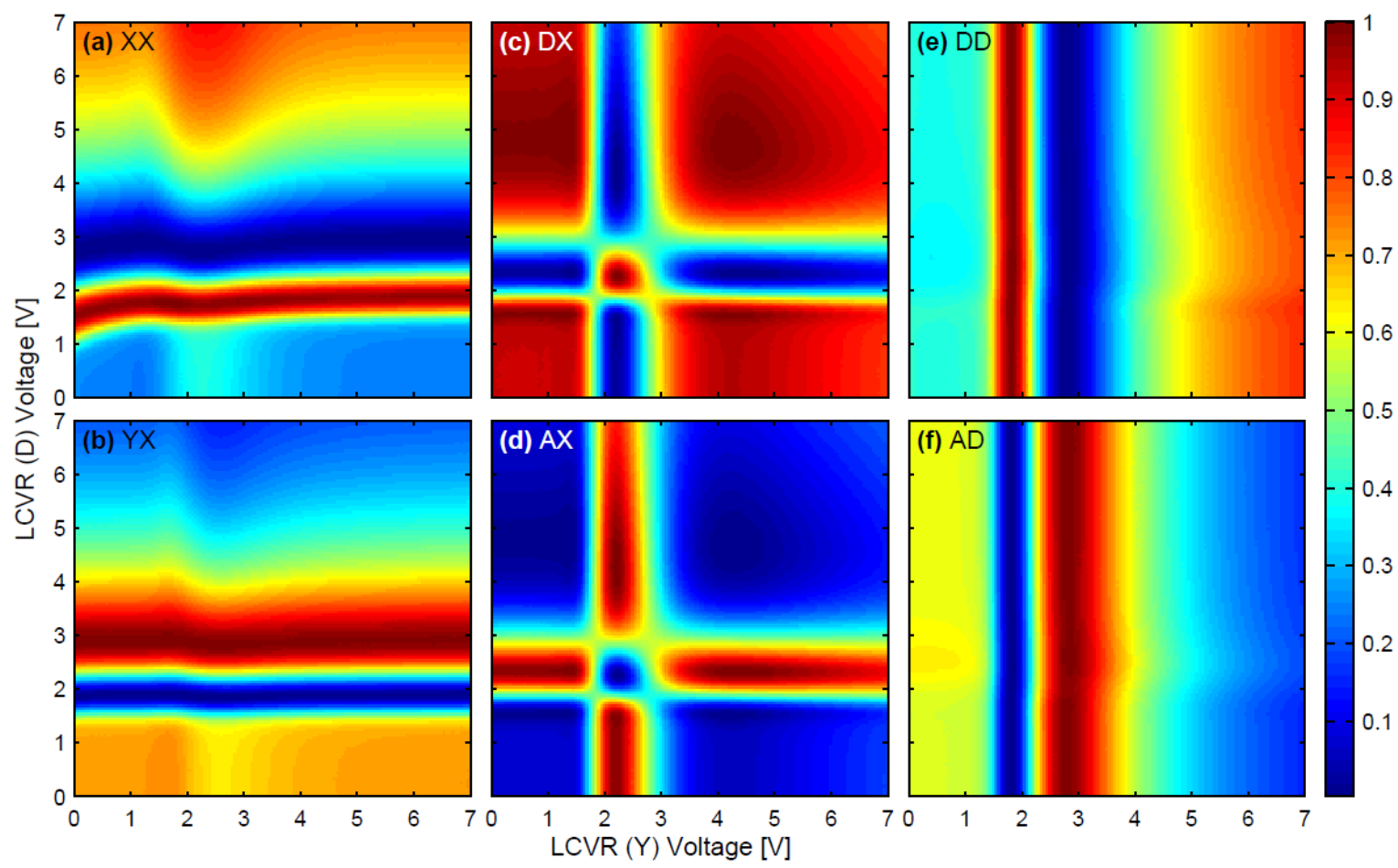

Figure A2.3 Six normalized intensity maps exploring the LCVR voltage space. Each map is defined by the specific polarizer-analyzer polarizations: (a) X-polarized incidence, X-polarized detection; (b) Y-polarized incidence, X-polarized detection; (c) D-polarized incidence, X-polarized detection; (d) A-polarized incidence, X-polarized detection; (e) D-polarized incidence, D-polarized detection; and (f) A-polarized incidence, Dpolarized detection. Each voltage axis consists of 140 steps with $50 \mathrm{mV} / \mathrm{step}$, and each data point is an average of 200 readings from the photodiode. 


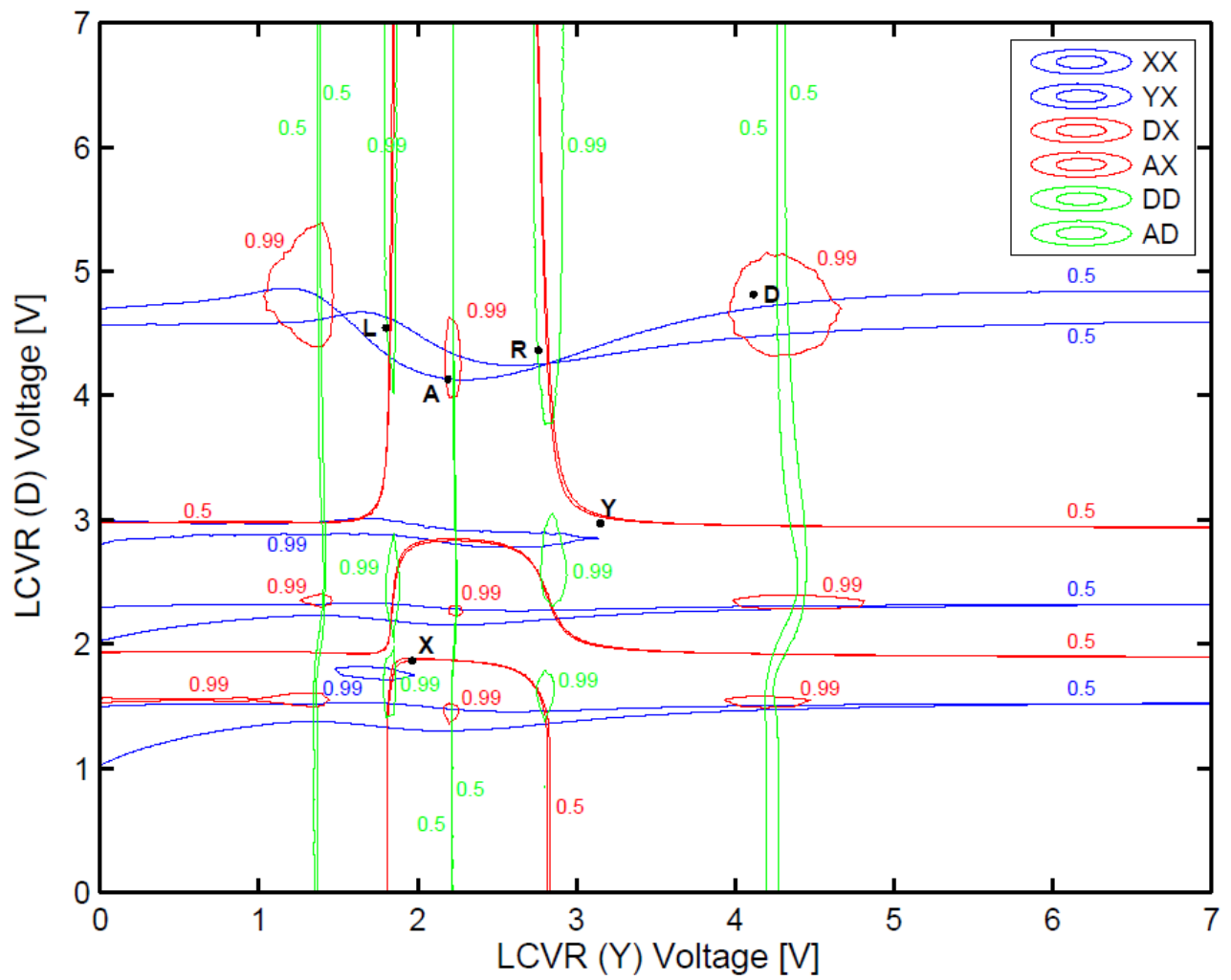

Figure A2.4 50\% and 99\% contour map based on the intensity maps shown in Figure A2.3. The optimal voltage pairs are located by the black dots. 


\section{Appendix A2.3 Matlab code for extracting second-order correlation function}

This appendix provides the home-written Matlab code for extracting the cross-correlation function $\left[g^{(2)}(\tau)\right]$ from time-tagged photon events acquired by two single-photon avalanche photon diodes ( $\tau$-SPADs). Conventionally, this calculation is done by histogramming the time differences between arrival times of any two photons, and then normalizing the whole curve to the occurrence at large correlation time. However, this linear algorithm is computation resource demanding, especially for the calculation at large correlation time, because the number of calculations to be done is proportional to the factorial of the number of photons in consideration. In fact, the maximum correlation time we managed to deal with is on the order of millisecond by using linear algarithm. At this time-scale, the value of the correlation function extracted from our data is still changing, rather than being an asymptotic constant as a function of the correlation time. Therefore, longer correlation time has to be considered. The calculation is enabled by employing multiple-tau algorithm, where the photon events are firstly histogrammed to binned intensities at different times, and then the correlation is calculated between these binned intensities. By varying the bin size exponentially, a second-order correlation function with logarithmically varying time can be extracted, as shown in references [125] and [145].

This script reads in a .ptu file generated by in-built software PicoHarp (PicoQuant, Inc). Each ptu file consists of a header (3632 bytes) and binary records that stores the arrival time of each detected photon. Each record is 32-bits long, where the lowest 28 bits records the time, and the next 4 bits stores the channel number. Channel number of 15 is reserved for overflow flag of the clock, namely that the clock rewinds after this record and restart ticking from zero. The absolute time of each photon event thus can be obtained by counting the number of overflows before the event and then summing up all the time. After this, we can calculate the correlation with multiple-tau algorithm.

Input parameters:

fid $=$ ptu file ID

NumberOfRecords $=$ total number of records

BinSettings $=$ [tauresmin, tauresmax, BINrt, BINum]:

tauresmin Smallest correlation time [pico-second]

tauresmax Largest correlation time [pico-second]

BINrt Bin width multiplication factor

BINum Number of bins in each group [NOTE: BINnum/BINrt $>=7$ is preferred]

Options $=3$ field structure

Options.delay Delay time between channel 0 and channel 1 [nano-second]

Options.Nread Number of records rad in per loop

Options.updateProgress plot $\mathrm{g}(2)$ as parsing though the data

Output parameters:

$\mathrm{H}=$ structure saving parameters used for extraction and the extracted results:

H.tauresmin Smallest correlation time [pico-second]

H.tauresmaxset Largest set correlation time [pico-second] 


$\begin{array}{ll}\begin{array}{l}\text { H.tauresmaxreal } \\ \text { H.tauresUnit }\end{array} & \text { Largest actual correlation time [pico-second] } \\ \text { H.BINratio } & \text { Bin width multiplication factor } \\ \text { H.chandelay } & \text { Delay time between channel } 0 \text { and channel 1 } \\ \text { H.chandelayUnit } & \text { Unit of the channel delay } \\ \text { H.xtau } & \text { correlation time vector } \tau \\ \text { H.xtaUnit } & \text { units of correlation time } \\ \text { H.yncorr } & \text { extracted correlation (normalized) } \\ \text { H.yerror } & \text { uncertainty in the extracted correlation }\end{array}$

function $\mathrm{H}=$ ExtractXg2_mtau(fid, NumberOfRecords, BinSettings, Options)

tauresmin $=$ BinSettings $(1)$;

tauresmax $=$ BinSettings $(2)$;

BINrt = BinSettings $(3)$;

BINum = BinSettings $(4)$;

delay = Options.delay;

Nread $=$ Options. Nread;

updateProgress = Options. updateProgress;

groupnum $=1+\operatorname{ceil}(\log ($ tauresmax $/$ tauresmin $) / \log ($ BINrt $))$;

tauresmaxreal $=$ tauresmin*BINrt^ (groupnum -1$) ; \quad \%$ Actual maximum tau-bin width (ps)

BINum2nd = BINum - BINum/BINrt; $\%$ Bin number in second group in the correlator

\% time vector xtau (in second) for plotting $g(2)$ :

xtau $=$ tauresmin. ${ }^{*}$ BINrt. ${ }^{\wedge}$ reshape $($ meshgrid $(1:$ groupnum $-1,1:$ BINum2nd $),[], 1)$;

xtau $=\operatorname{cat}\left(1\right.$, tauresmin. $*^{\text {ones }}($ BINum, 1$), x$ tau $)$;

xtau $=$ cumsum $(x \operatorname{tau}) \cdot * 1 \mathrm{e}-12 ; \%[$ second $]$

\% logical array to extract correlation vector out of correlator matrix

tlogic $=\operatorname{logical}([$ ones $($ BINum, 1$) \operatorname{repmat}([$ zeros $($ BINum-BINum2nd, 1$) ;$ ones (BINum2nd, 1) ], 1, groupnum-1)] );

chandelay $=$ delay*250; $\%$ Change the unit of delay into clock ticks ( $4 \mathrm{ps} / \mathrm{click}$ )

WRAPAROUND $=210698240$;

if updateProgress $==1$ 


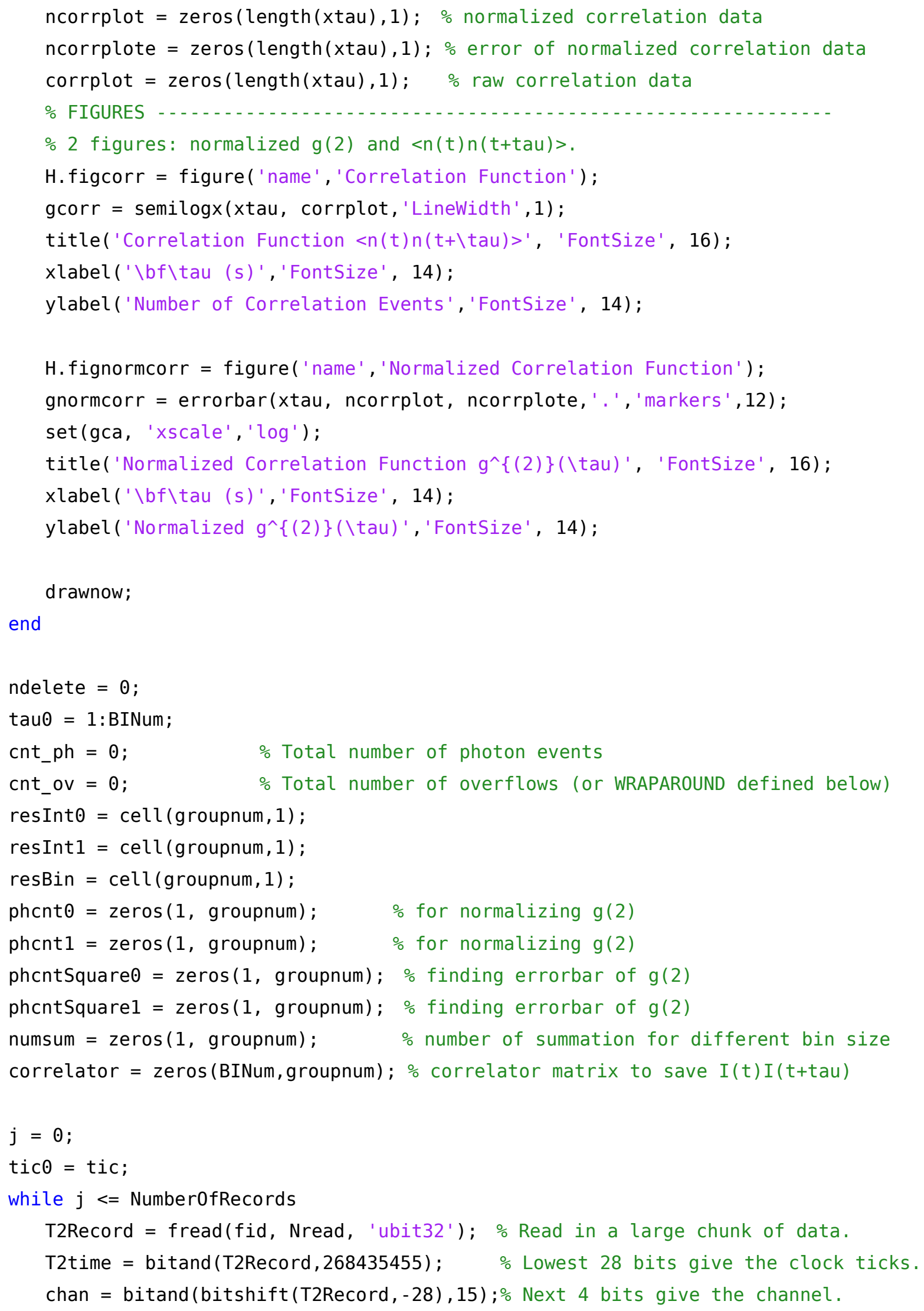




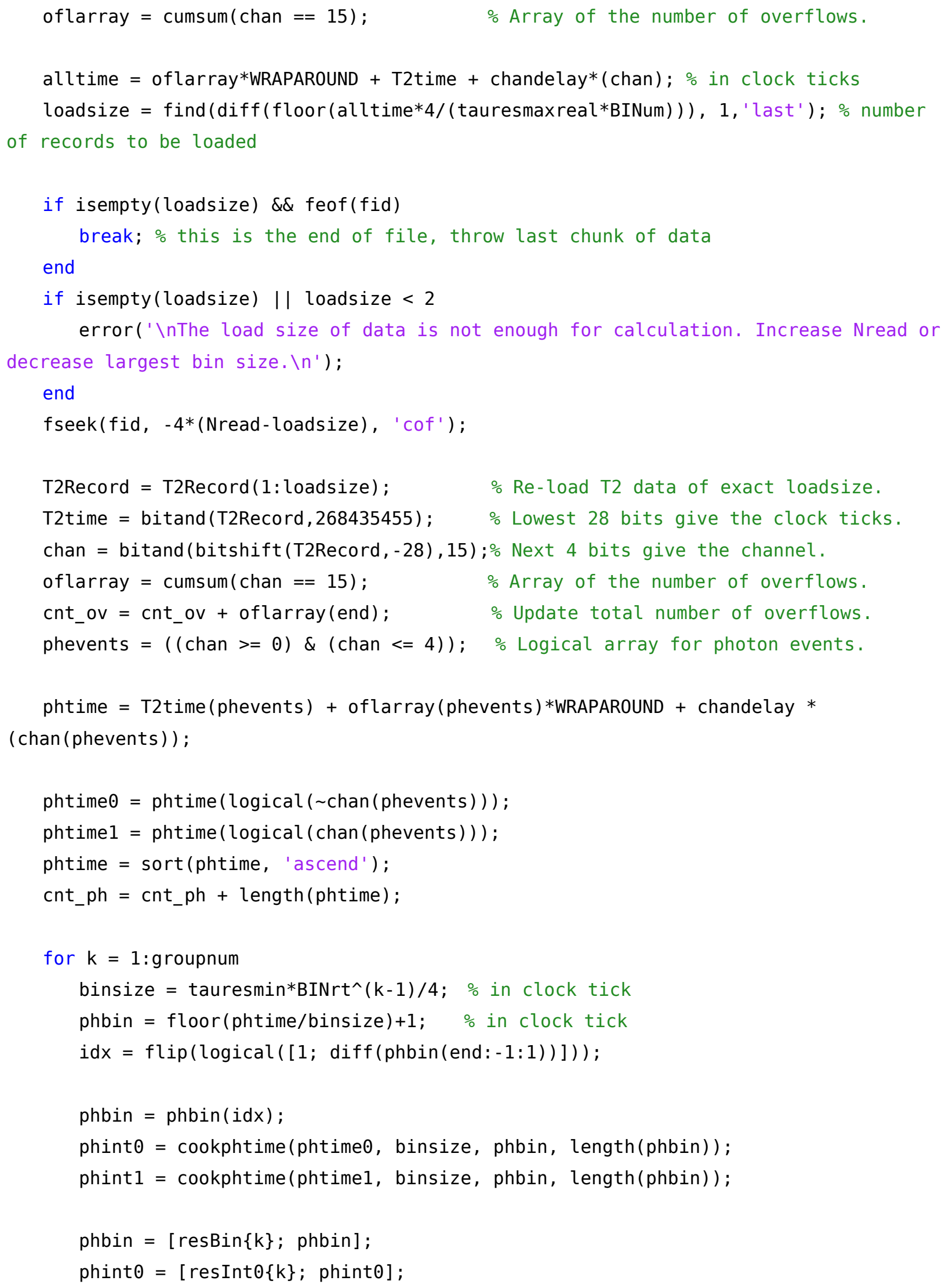




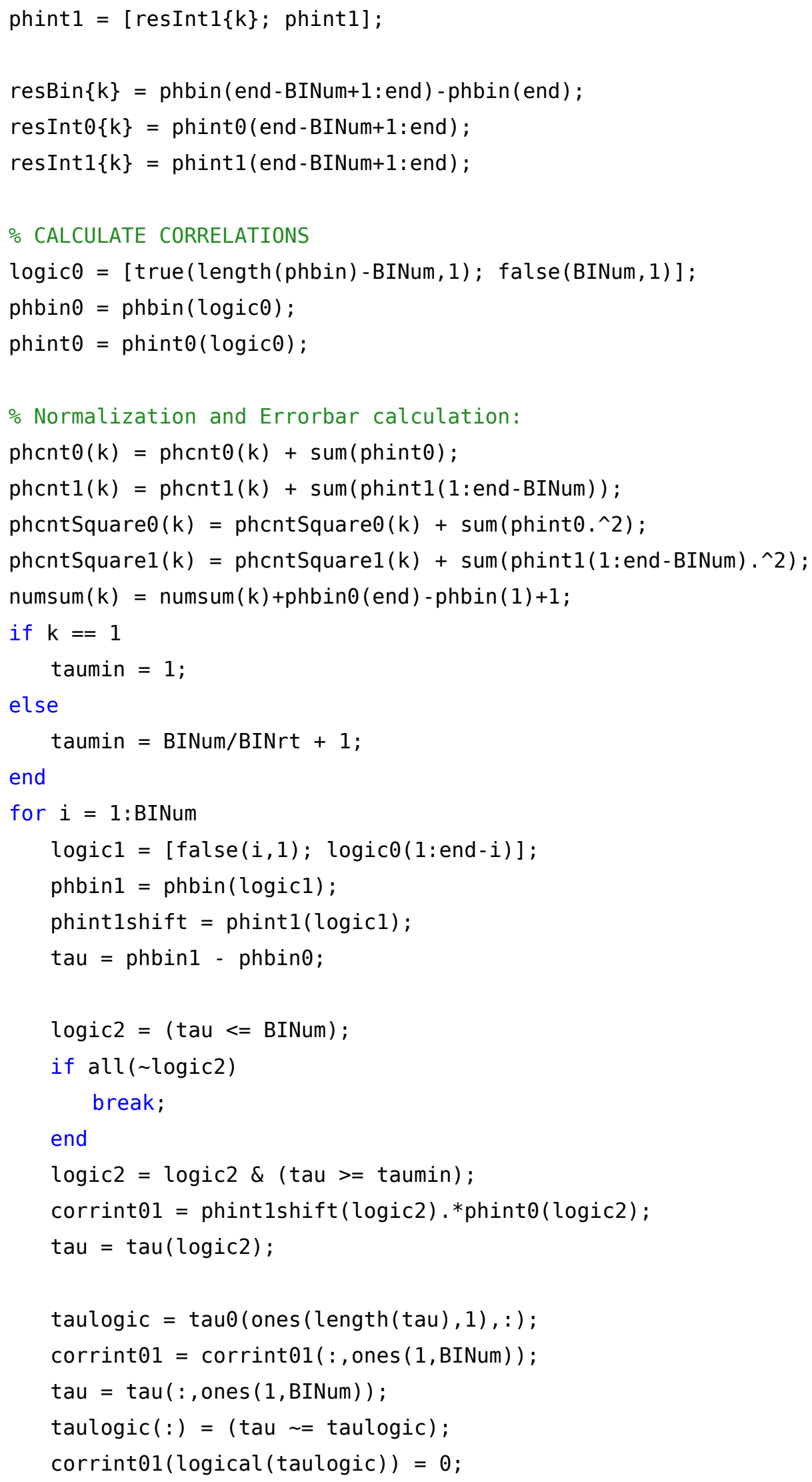




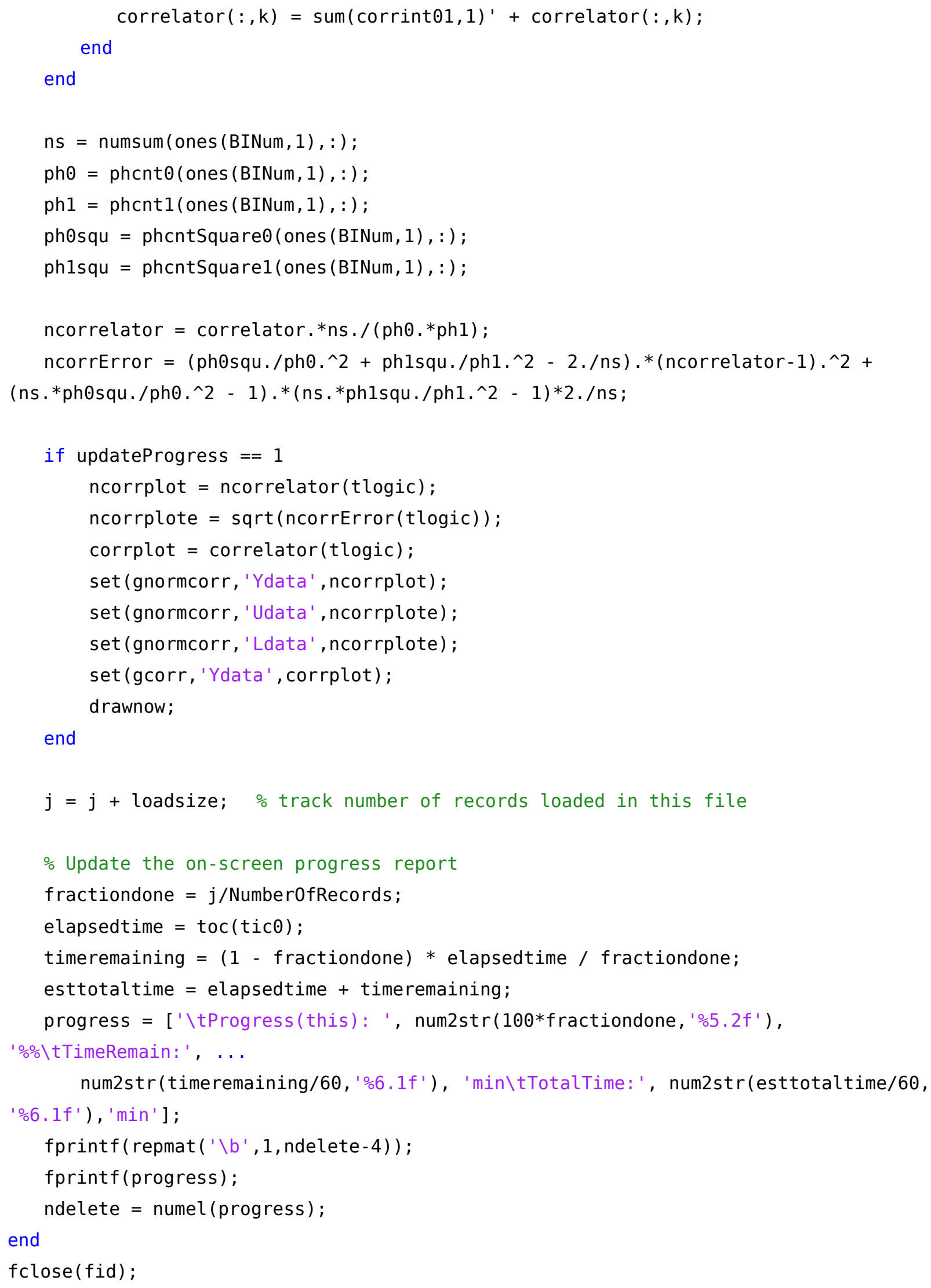




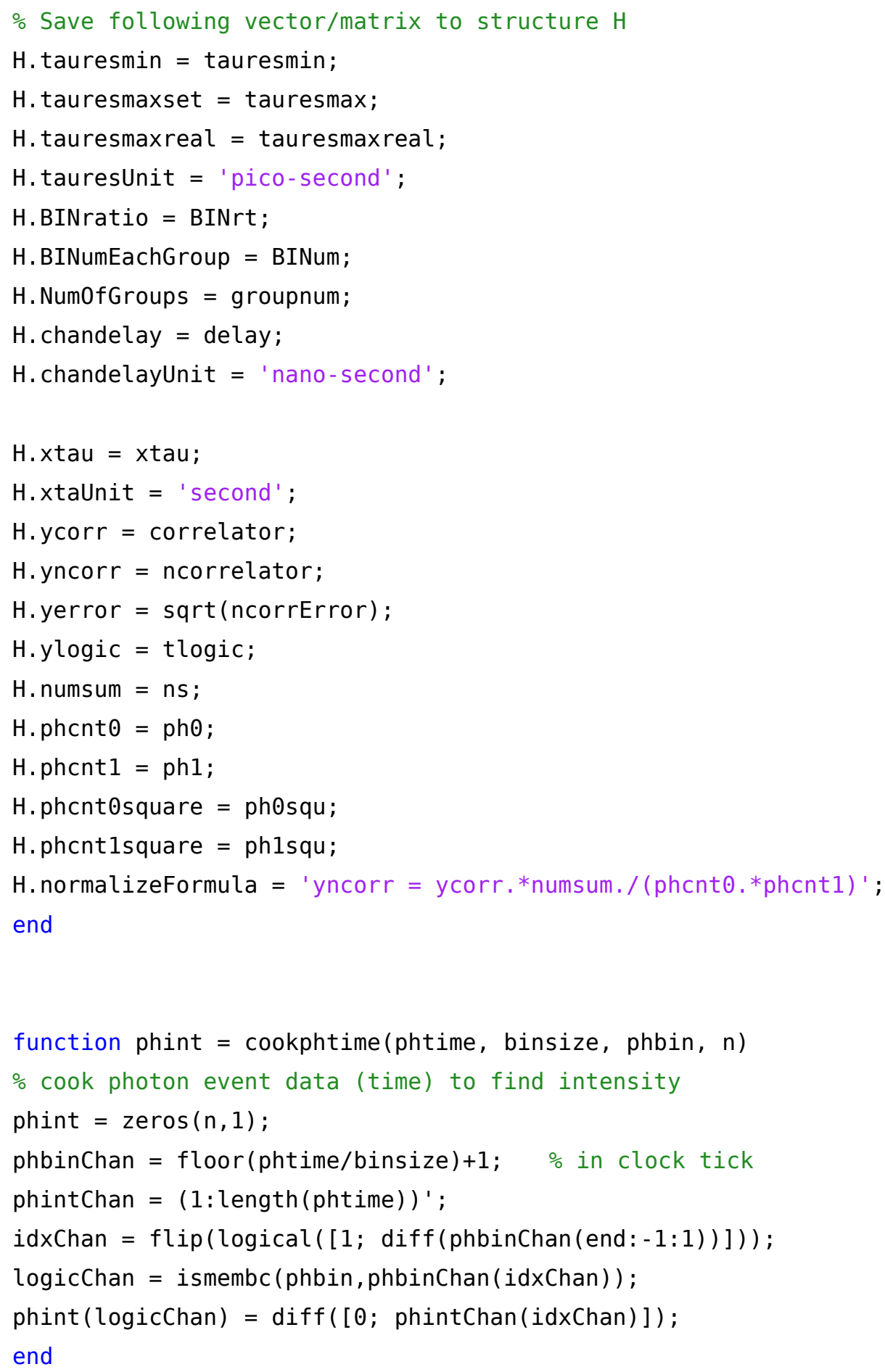




\section{Appendix A3.1 Mueller matrix of the collection path}

The effect on polarization by PL collection path is described in the framework of Mueller calculus,

$$
S_{f}=M_{c o l} S_{i}
$$

where $S_{i}$ and $S_{f}$ are the 4-by-1 Stokes vector representing the initial and final polarization state of the beam defined in Eqn. (3.34), and $M_{c o l}$ is the Mueller matrix of the collection path. In principle, $M_{c o l}$ can be determined by one pair of known $\left[S_{i}, S_{f}\right]$ via Moore-Penrose pseudoinverse:

$$
M_{\text {col }}=S_{f} S_{i}^{+}
$$

where $S_{i}^{+}$is the pseudoinverse (or generalized inverse) of matrix $S_{i}$. It can be calculated by following formula, provided matrix $\left(S_{i} S_{i}^{\star}\right)$ is invertible,

$$
S_{i}^{+}=S_{i}^{\star}\left(S_{i} S_{i}^{\star}\right)^{-1}
$$

where $S_{i}^{\star}$ is the Hermitian transpose (conjugate transpose ) of $S_{i}$. This is possible for a general $S_{i}$. However, the $M_{c o l}$ obtained by this way is only optimized for one single specific $S_{i}$, and significant error is introduced for other polarization measurements. To overcome this issue, we measured 15 pairs of $\left[S_{i}, S_{f}\right]$ with different $S_{i}$ that "evenly" sample the whole polarization sphere (Poincare Sphere), and then simultaneously fit them by using Eqn. (A3.1) with all 16 elements of $M_{c o l}$ as free parameters. In measurement, the laser beam is fixed at $928.3885 \mathrm{~nm}$, and a combination of a half wave plate (HWP) and a quarter wave plate (QWP) is used to produce different initial polarizations. The finally determined Mueller matrix $M_{c o l}$ is

$$
M_{\text {col }}=\left(\begin{array}{cccc}
0.7480 & 0.0780 & -0.0099 & -0.0137 \\
0.0807 & 0.7217 & 0.0247 & -0.1532 \\
-0.0170 & -0.0775 & -0.2200 & -0.6796 \\
-0.0021 & 0.0209 & 0.6569 & -0.2138
\end{array}\right)
$$

with the uncertainty expressed in terms of percentage as

$$
\frac{\sigma_{M}}{\left|M_{\text {col }}\right|}=\left(\begin{array}{cccc}
0.08 \% & 1.45 \% & 9.52 \% & 9.01 \% \\
0.28 \% & 0.10 \% & 1.28 \% & 0.18 \% \\
0.73 \% & 0.19 \% & 0.15 \% & 0.11 \% \\
5.06 \% & 0.77 \% & 0.10 \% & 0.12 \%
\end{array}\right)
$$

Besides the experimental errors, the other error source is the LCVRs, which contribute a significant portion of observed error via its voltage-dependent shift of fast axis (see Section 2.3), and the small fluctuation of fast axis location from run to run (see Appendix A2.2). 


\section{Appendix A3.2 Double 2-level model}

Motivated by the classical model that regards each transition in a neutral QD as a harmonic oscillator, we replace each oscillator by a 2-level system in double 2-level model. This forms a composite system that consists of two 2-level structures denoted as $a$ and $b$, respectively. The density matrix of the entire system is thus constructed from the tensor product of the density matrices of the two sub-systems,

$$
\rho^{(a b)}=\rho^{(a)} \otimes \rho^{(b)}
$$

The time-evolution of $\rho^{(a b)}$ is still governed by the Schrodinger equation

$$
i \hbar \frac{d}{d t} \rho^{(a b)}=\left[H_{a b}, \rho^{(a b)}\right]
$$

where $H_{a b}=H_{a} \otimes I_{b}+I_{a} \otimes H_{b}$ is the Hamiltonian of the whole system, $H_{a}=e \boldsymbol{d}_{1} \cdot \boldsymbol{E}_{0} \cos (\omega t)$ and $H_{b}=$ $e \boldsymbol{d}_{2} \cdot \boldsymbol{E}_{0} \cos (\omega t)$ are the electric dipole energy of each 2-level system interacting with the excitation field $\boldsymbol{E}_{0} \cos (\omega t)$. Since $H_{a b}$ does not have any term to couple the two sub-systems, the time evolution of $\rho^{(a b)}$ is thus determined by the individual evolution of each constituent system. This means that we can solve Eqn. (A3.7) by working in each subspace. For example, the time-evolution of two-level $a$ is dictated by super-matrix $\boldsymbol{M}_{a}$ with following form:

$$
\mathcal{M}_{a}=\left(\begin{array}{cccc}
-\Gamma_{s p} & 0 & i \Omega_{a} / 2 & -i \Omega_{a} / 2 \\
\Gamma_{s p} & 0 & -i \Omega_{a} / 2 & i \Omega_{a} / 2 \\
i \Omega_{a} / 2 & -i \Omega_{a} / 2 & -i \delta_{a}-\Gamma & 0 \\
-i \Omega_{a} / 2 & i \Omega_{a} / 2 & 0 & i \delta_{a}-\Gamma
\end{array}\right)
$$

written in the basis of $\tilde{\rho}_{11}^{(a)}, \tilde{\rho}_{00}^{(a)}, \tilde{\rho}_{10}^{(a)}, \tilde{\rho}_{01}^{(a)}$. Here, $\Gamma_{\mathrm{sp}}$ is population decay rate, $\Gamma$ is total dephasing rate, $\Omega_{a}$ is the Rabi frequency associated with the transition $a$, and $\delta_{a}=\omega-\omega_{a}$ is the excitation detuning with respect to transition frequency $\omega_{a}$. Considering continuous wave excitation, the steady state solution is

$$
\begin{aligned}
& \tilde{\rho}_{11}^{(a)}=\frac{1}{2} \frac{\left(\Gamma / \Gamma_{\mathrm{sp}}\right) \Omega_{a}^{2}}{\left(\Gamma / \Gamma_{\mathrm{sp}}\right) \Omega_{a}^{2}+\Gamma^{2}+\delta_{a}^{2}} \\
& \tilde{\rho}_{00}^{(a)}=\frac{1}{2} \frac{\left(\Gamma / \Gamma_{\mathrm{sp}}\right) \Omega_{a}^{2}+2 \Gamma^{2}+2 \delta_{a}^{2}}{\left(\Gamma / \Gamma_{\mathrm{sp}}\right) \Omega_{a}^{2}+\Gamma^{2}+\delta_{a}^{2}} \\
& \tilde{\rho}_{10}^{(a)}=\frac{1}{2} \frac{\left(\delta_{a}-i \Gamma\right) \Omega_{a}}{\left(\Gamma / \Gamma_{\mathrm{sp}}\right) \Omega_{a}^{2}+\Gamma^{2}+\delta_{a}^{2}} \\
& \tilde{\rho}_{01}^{(a)}=\frac{1}{2} \frac{\left(\delta_{a}+i \Gamma\right) \Omega_{a}}{\left(\Gamma / \Gamma_{\mathrm{sp}}\right) \Omega_{a}^{2}+\Gamma^{2}+\delta_{a}^{2}}
\end{aligned}
$$

Similar expression can be obtained for sub-system $b$ by replacing all subscript $a$ with $b$. The final scattered PL intensity from the whole system can found as (the similar method as used in 3-level model),

$$
\begin{gathered}
\bar{I}_{X}^{\text {Double }}=I_{0}\left\{\tilde{\rho}_{11}^{(a)} \sin ^{2}(\varphi)+\tilde{\rho}_{11}^{(b)} \cos ^{2}(\varphi)-\mathfrak{R e}\left[\left\langle\pi_{a}^{\dagger} \pi_{b}\right\rangle\right] \sin (2 \varphi)\right\} \\
\bar{I}_{Y}^{\text {Double }}=I_{0}\left\{\tilde{\rho}_{11}^{(a)} \sin ^{2}(\varphi)+\tilde{\rho}_{11}^{(b)} \cos ^{2}(\varphi)+\mathfrak{R e}\left[\left\langle\pi_{a}^{\dagger} \pi_{b}\right\rangle\right] \sin (2 \varphi)\right\} \\
\bar{I}_{D}^{\text {Double }}=\frac{I_{0}}{2}\left\{\tilde{\rho}_{11}^{(a)}+\tilde{\rho}_{11}^{(b)}+\left(\tilde{\rho}_{11}^{(a)}-\tilde{\rho}_{11}^{(b)}\right) \sin (2 \varphi)-2 \Re e\left[\left\langle\pi_{a}^{\dagger} \pi_{b}\right\rangle\right] \cos (2 \varphi)\right\} \\
\bar{I}_{A}^{\text {Double }}=\frac{I_{0}}{2}\left\{\tilde{\rho}_{11}^{(a)}+\tilde{\rho}_{11}^{(b)}-\left(\tilde{\rho}_{11}^{(a)}-\tilde{\rho}_{11}^{(b)}\right) \sin (2 \varphi)+2 \mathfrak{R e}\left[\left\langle\pi_{a}^{\dagger} \pi_{b}\right\rangle\right] \cos (2 \varphi)\right\}
\end{gathered}
$$




$$
\begin{aligned}
& \bar{I}_{L}^{\text {Double }}=\frac{1}{2} I_{0}\left\{\tilde{\rho}_{11}^{(a)}+\tilde{\rho}_{11}^{(b)}+2 \operatorname{Im}\left[\left\langle\pi_{a}^{\dagger} \pi_{b}\right\rangle\right]\right\} \\
& \bar{I}_{R}^{\text {Double }}=\frac{1}{2} I_{0}\left\{\tilde{\rho}_{11}^{(a)}+\tilde{\rho}_{11}^{(b)}-2 \operatorname{Im}\left[\left\langle\pi_{a}^{\dagger} \pi_{b}\right\rangle\right]\right\}
\end{aligned}
$$

where the intensity constant $I_{0}$ is defined in Eqn. (3.14), and the ensemble average of $\pi_{a}^{\dagger} \pi_{b}$ is

$$
\left\langle\pi_{a}^{\dagger} \pi_{b}\right\rangle=\operatorname{Tr}\left\{\pi_{a}^{\dagger} \pi_{b} \rho\right\}=\operatorname{Tr}\left\{\pi_{a}^{\dagger} \rho^{(a)}\right\} \cdot \operatorname{Tr}\left\{\pi_{b} \rho^{(b)}\right\}=\tilde{\rho}_{01}^{(a)} \tilde{\rho}_{10}^{(b)}
$$

where symbol $\mathrm{Tr}$ represents trace evaluation.

By doing a global fitting to the data in Figure 3.3(a-b) with Eqns. (A3.10) (A3.11), we obtain a new set of parameters for QD1, which is then used to calculate $\mathfrak{R e}\left[\left\langle\pi_{a}^{\dagger} \pi_{b}\right\rangle\right]$ and plotted in green curves as shown in Figure 3.5 . 


\section{Appendix A4.1 Rate model for the above-band excitation}

With solely above-band excitation, the photoluminescence of a QD can only be achieved by capturing the free charge carriers from the local environment, as shown in Figure A4.1. Here, only four QD charge complex are considered, i.e., no charge, one electron, one hole and neutral exciton, because no trion or biexciton emission is observed in the emission spectrum, as discussed in Appendix A2.1. The population of each QD state obeys the following first-order differential equations

$$
\begin{aligned}
& \frac{d \mathrm{~N}_{11}}{d t}=-\Gamma_{\mathrm{sp}} \mathrm{N}_{11}+\gamma_{\mathrm{e}} \mathrm{N}_{01}+\gamma_{\mathrm{h}} \mathrm{N}_{10}+\gamma_{X} \mathrm{~N}_{00} \\
& \frac{d \mathrm{~N}_{10}}{d t}=\gamma_{\mathrm{e}} \mathrm{N}_{00}-\gamma_{h} \mathrm{~N}_{10} \\
& \frac{d \mathrm{~N}_{01}}{d t}=\gamma_{h} \mathrm{~N}_{00}-\gamma_{\mathrm{e}} \mathrm{N}_{01} \\
& \frac{d \mathrm{~N}_{00}}{d t}=-\left(\gamma_{\mathrm{e}}+\gamma_{\mathrm{h}}+\gamma_{X}\right) \mathrm{N}_{00}+\Gamma_{\mathrm{sp}} \mathrm{N}_{11}
\end{aligned}
$$

where $\mathrm{N}_{\mathrm{jk}}$ is the population of the QD state with $\mathrm{j}$ electrons and $\mathrm{k}$ holes, $\Gamma_{\mathrm{sp}}$ is the recombination rate of the neutral exciton, $\gamma_{\mathrm{e}}, \gamma_{\mathrm{h}}$, and $\gamma_{X}$ are the capturing rate of electron, hole or exciton from the environment. The populations are normalized, $\mathrm{N}_{11}+N_{01}+\mathrm{N}_{10}+\mathrm{N}_{00}=1$. Thus the steady state solution of the neutral exciton population is

$$
\mathrm{N}_{11}=\left\{1+\left(1+\frac{\gamma_{\mathrm{e}}}{\gamma_{\mathrm{h}}}+\frac{\gamma_{\mathrm{h}}}{\gamma_{\mathrm{e}}}\right) \frac{\Gamma_{\mathrm{sp}}}{\gamma_{\mathrm{e}}+\gamma_{\mathrm{h}}+\gamma_{X}}\right\}^{-1}
$$

Since all charge capturing rates are proportional to the density of the free charge carriers, which in further proportional to the excitation power of the above-band laser, therefore the capturing rates are proportional to the excitation power, i.e., $\gamma_{\mathrm{e}} \propto P, \gamma_{\mathrm{h}} \propto P$, and $\gamma_{\mathrm{X}} \propto P$. Eqn. (A4.2) can be rewritten as

$$
\mathrm{N}_{11}=\mathrm{A} \frac{\mathrm{P}}{\mathrm{P}_{\mathrm{S}}+\mathrm{P}}
$$

where $\mathrm{A}$ is a power-independent constant, $\mathrm{P}$ is the laser power, and $\mathrm{P}_{\mathrm{S}}$ is the saturation power. Eqn. (A4.3) shares the same functional form of the first term in Eqn. (4.5).

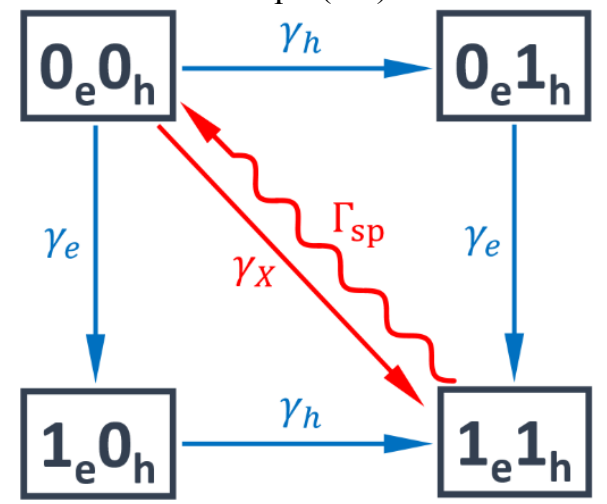

Figure A4.1 The evolution of the QD state by capturing free charge carriers from local environment. The state is represented by square box, labeled by the number of confined charge carriers. $\gamma_{\mathrm{e}}$ is the electron capture rate, $\gamma_{\mathrm{e}}$ is the hole capture rate, and $\gamma_{\mathrm{X}}$ is the exciton capture rate, and $\Gamma_{\mathrm{sp}}$ is the radiative recombination rate. 


\section{Appendix A4.2 Temperature rising estimation}

Generally, the thermal diffusion in semiconductor with constant thermal conductivity is dictated by

$$
\frac{1}{\alpha} \frac{\partial \mathrm{T}}{\partial t}=\frac{g_{q}}{k}+\nabla^{2} \mathrm{~T}
$$

where $\mathrm{T}$ is the material temperature, $\alpha$ is thermal diffusivity $\left[\mathrm{m}^{2} / \mathrm{s}\right], k$ is thermal conductivity $[\mathrm{W} /(\mathrm{m} \cdot \mathrm{K})]$, and $g_{q}$ is the heat source (heat generation density) $\left[\mathrm{J} / \mathrm{m}^{3}\right]$. The thermal diffusivity $\alpha$ is related to thermal conductivity $k$ by $\alpha=k /\left(C_{p} \cdot \rho\right)$, where $C_{p}$ is the specific heat of the material, and $\rho$ is the density. For steady state solution, no temperature varies over time,

$$
\nabla^{2} \mathrm{~T}=-\frac{g_{q}}{k}
$$

In our case, the heat source is the absorption of the above-band laser light (at $632 \mathrm{~nm}$ ). Although part of the absorbed energy is released to the environment via fluorescence, let's ignore it and assume that all the absorbed energy is turned into heat. Thus, the energy absorbed by the medium GaAs (with the refractive index $\mathrm{n}$ and absorption coefficient $\beta$ ) per unit volume is

$$
g_{q}=\frac{\mathrm{P}}{\pi r_{0}^{2} d}(1-R)\left(1-e^{-\beta d}\right)=\frac{\mathrm{P}}{\pi r_{0}^{2} d}\left\{1-\left(\frac{n-1}{n+1}\right)^{2}\right\}\left(1-e^{-\beta d}\right)
$$

where $\mathrm{P}$ is the laser power at the surface of the sample, $R$ is the reflectivity of GaAs, $\beta$ is the absorption coefficient of GaAs at $632 \mathrm{~nm}, d$ is the thickness of the sample, and $r_{0}$ is the radius of the focused laser spot. Rewrite Eqn. (A4.5) in cylindrical coordinate and notice that the absorption of Eqn. (A4.6) only occurs in the range of $r \leq r_{0}$,

$$
\frac{1}{r} \frac{\partial}{\partial r}\left(r \frac{\partial}{\partial r}\right) \mathrm{T}=-\frac{g_{q}}{k} H\left(r-r_{0}\right)
$$

where $H(r)$ is the Heaviside step function. The general solution is

$$
\begin{aligned}
& \mathrm{T}_{<r_{0}}(r)=-\frac{g_{q}}{4 k} r^{2}+C \\
& \mathrm{~T}_{>r_{0}}(r)=-\frac{g_{q} r_{0}^{2}}{4 k}\left(1+2 \ln \frac{r}{r_{0}}\right)+C
\end{aligned}
$$

This gives that the temperature at the center of the focusing spot (where the QD locates) is $C$. If we assume the edge of the sample is held at constant temperature as heat sink, the temperature difference between the center spot to the edge of the sample is

$$
\Delta \mathrm{T}=-\frac{g_{q} r_{0}^{2}}{4 k}\left(1+2 \ln \frac{r_{s}}{r_{0}}\right)
$$

where $r_{s}$ is the lateral size of the sample. The largest laser power used in RPLE experiment is $\mathrm{P}=200 \mathrm{nW}$. The thickness of GaAs spacer is $d=1 \mu \mathrm{m}$, and refractive index is $\mathrm{n}=3.8$ at $632 \mathrm{~nm}$. The lateral size of the sample is $r_{s}=5 \mathrm{~mm}$. Other constants are found at Landolt-Börnstein online database: absorption coefficient at 632 $\mathrm{nm}$ is $\beta=4 \times 10^{4} / \mathrm{cm}$ and thermal conductivity $\mathrm{k}=10 \mathrm{Wm}^{-1} \mathrm{~K}^{-1}$. Plug in these parameters, we find

$$
\Delta \mathrm{T}=-\frac{0.658 P}{4 \pi k d}\left(1+2 \ln \frac{r_{s}}{r_{0}}\right)=0.15 \mathrm{mK}
$$


Note the final solution Eqn. (A4.10) is not physical when the sample size $r_{s}$ goes to big. In such case, 2D model is not proper anymore, because the heat can leave the sample along $\mathrm{z}$ direction, rather than just spreading in the horizontal plane of the sample. A 3-dimensional simulation is needed for that case. 